\title{
Implementation strategy for the school-based sex education program Long Live Love
}

Citation for published version (APA):

Schutte, L. (2017). Implementation strategy for the school-based sex education program Long Live Love: A Dynamic Process. [Doctoral Thesis, Maastricht University]. Maastricht University. https://doi.org/10.26481/dis.20170712ls

Document status and date:

Published: 01/01/2017

DOI:

$10.26481 /$ dis.20170712ls

Document Version:

Publisher's PDF, also known as Version of record

\section{Please check the document version of this publication:}

- A submitted manuscript is the version of the article upon submission and before peer-review. There can be important differences between the submitted version and the official published version of record.

People interested in the research are advised to contact the author for the final version of the publication, or visit the DOI to the publisher's website.

- The final author version and the galley proof are versions of the publication after peer review.

- The final published version features the final layout of the paper including the volume, issue and page numbers.

Link to publication

\footnotetext{
General rights rights.

- You may freely distribute the URL identifying the publication in the public portal. please follow below link for the End User Agreement:

www.umlib.nl/taverne-license

Take down policy

If you believe that this document breaches copyright please contact us at:

repository@maastrichtuniversity.nl

providing details and we will investigate your claim.
}

Copyright and moral rights for the publications made accessible in the public portal are retained by the authors and/or other copyright owners and it is a condition of accessing publications that users recognise and abide by the legal requirements associated with these

- Users may download and print one copy of any publication from the public portal for the purpose of private study or research.

- You may not further distribute the material or use it for any profit-making activity or commercial gain

If the publication is distributed under the terms of Article $25 \mathrm{fa}$ of the Dutch Copyright Act, indicated by the "Taverne" license above, 


\section{IMPLEMENTATION STRATEGY FOR THE SCHOOL-BASED SEX EDUCATION PROGRAM LONG LIVE LOVE: A DYNAMIC PROCESS}


ISBN:

Layout:

Printing:

Cover design:
978-94-6299-626-7

Design Your Thesis | designyourthesis.com

Ridderprint | ridderprint.nl

Ontwerphaven | ontwerphaven.nl

Copyright $\odot 2017$ Lisette Schutte. No part of this dissertation may be reproduced or transmitted in any form or by any means without written permission from the author. The work in this dissertation was performed within the Department of Work and Social Psychology, Faculty of Psychology and Neuroscience, Maastricht University, in collaboration with STI Aids, the Netherlands (Soa Aids Nederland). This project was supported by The Netherlands Organization for Health Research and Development (ZonMw), grant number 124280004 . 


\title{
IMPLEMENTATION STRATEGY FOR THE SCHOOL-BASED SEX EDUCATION PROGRAM LONG LIVE LOVE: A DYNAMIC PROCESS
}

\author{
DISSERTATION
}

to obtain the degree of Doctor at Maastricht University,

on the authority of the Rector Magnificus Prof. dr. Rianne M. Letschert, in accordance with the decision of the Board of Deans, to be defended in public on Wednesday $12^{\text {th }}$ of July 2017 at 14.00 hours

by

\section{Lisette Schutte}




\section{Promotor}

Prof. dr. G. Kok

\section{Co-promotor}

Dr. F.E.F. Mevissen

\section{Assessment Committee}

Prof. dr. R.A.C. Ruiter, Chair

Prof. dr. S.P.J. Kremers

Prof. dr. I. Mesters

Prof. dr. G.S. Parcel, University of Texas at Houston

Dr. M.E.G. Wolfers, Municipal Health Service Rotterdam-Rijnmond 


\section{TABLE OF CONTENTS}

$\begin{array}{lll}\text { Chapter } 1 & \text { General introduction } & 7\end{array}$

Chapter 2 Long Live Love: The implementation of a school-based sex education 31 program in the Netherlands

Chapter 3 Support from the Municipal Health Services (MHS) in implementing school-based sex education: enabling and inhibiting factors in times of economic cutbacks

Chapter 4 Innovatively supporting teachers' implementation of school-based sex education: Developing a Web-based coaching intervention from problem to solution

Chapter 5 Effect evaluation of a Web-based coaching intervention to support 107 implementation of sex education among secondary school teachers: Randomized controlled trial

Chapter 6 Process evaluation of a Web-Based coaching intervention to support implementation of sex education among secondary school teachers

Chapter 7 General discussion

References

Valorization

Summary 209

Samenvatting

Acknowledgements 
CHAPTER 1

\section{General introduction}





\section{GENERAL INTRODUCTION}

"Change in education is easy to propose, hard to implement, and extraordinarily difficult to sustain' (Hargreaves \& Fink, 2006).

This dissertation describes the systematic theory- and evidence-based development and evaluation of an implementation strategy for the optimal adoption, implementation and continuation of a secondary school-based sex education program, 'Long Live Love' - 'Lang Leve de Liefde (LLL), in the Netherlands. This strategy is aimed at the target group of secondary school teachers as well as the intermediary health promotion professionals from the Municipal Health Services (MHS) who support teachers in school-based health promotion. In particular, the development and effects of the most innovative component of the implementation strategy is the focus of this dissertation. It is the web-based coaching intervention 'Lesgevenindeliefde.nl' or 'Teachinglove.nl' to enhance completeness and fidelity of LLL implementation by teachers. This project is a collaboration between Maastricht University and STI Aids Netherlands. STI Aids Netherlands is responsible for the development and implementation of interventions for the promotion of sexual health of the youth, including those in schools. The implementation strategy for LLL was developed involving teachers and the MHS. This introductory chapter provides the background and context of the studies presented in this dissertation. This chapter ends by outlining the dissertation.

\section{Importance of implementation}

The field of public health has seen interventions come and go, some with little or no impact. The lack of impact can be explained by lack of program quality. However, the success of a health promotion program is not only dependent on the quality and content of the program but also on the quality and extent of its implementation (Durlak $\&$ DuPre, 2008). Over the recent years, implementation has been acknowledged as an increasingly important aspect of health promotion. This is due to the increased awareness that if health promotion programs are not implemented systematically, their impact on public health will remain low (Durlak \& DuPre, 2008; Glasgow et al., 2012; Steckler \& Linnan, 2002). It has been established that programs are more likely to be successful if implemented completely and as prescribed by program developers (Bessems et al., 2011; Durlak \& Dupre, 2008; Payne, 2009; Payne, Gottfredson, \& Gottfredson, 2006). Variability in implementation is related to variability in achieving expected program outcomes (Lendrum \& Humphrey, 2012; Little, Riggs, Shin, Tate, \& Pentz, 2015). 
Implementation is considered a process consisting of different stages, namely dissemination (i.e. awareness), adoption (i.e. form an intention, uptake, initiate, commit, accept a program), implementation (i.e. initial use), and continuation (i.e. continued use) (Rogers, 2003). The implementation process thus refers to the sum total of each of these stages. Each stage in this process, including the implementation stage, are essential to promote for program success (Paulussen, Wiefferink, \& Mesters, 2007).

When developing health promotion programs, implementation, in its' entirety, is nevertheless often an aspect which is overlooked, insufficiently considered, not planned for or poorly conducted, regularly leading to program failure (Green \& Kreuter, 2005). The focus lies on developing the intervention itself, the content, and not on the subsequent use of it. Having an appealing intervention, however, is not enough to guarantee the widespread use of it. An intervention can have high content quality but will have minimal or no impact if it is not used, used inadequately or discontinued altogether (Bartholomew, Parcel, Kok, Gottlieb, \& Fernández, 2011). Additionally, the erroneous assumption is made that once the intervention is developed, it will automatically be used. Consequently, programs are not being implemented at all or are not always being implemented completely or in the way they were intended by program developers and certainly not implemented in the long run (Forman, Olin, Hoagwood, Crowe, \& Saka, 2009; Oldenburg \& Parcel, 2002). Non-used health programs are not only a waste of money but also make positive results less likely to be achieved. A focus on implementation is thus indispensable for the development and delivery of successful health promotion programs.

How can one ensure that an intervention is used and thus reaches the target group? To seal the gap between development and use of health promotion programs and ensure optimal implementation, program developers need to plan and actively execute the implementation plan. On the one hand, they can anticipate on implementation by developing programs that are congruent with the preferences, needs and possibilities of its users (youngsters, teachers) and the intervention context (education system, schools, MHS - culture, social-political context, consensus). Implementation is thus already considered during program development and not solely upon program completion. On the other hand, program developers can actively intervene in the adoption- and implementation process with theory- and evidence-based implementation strategies, including trainings and technical support (Rohrbach, Grana, Sussman, \& Valente, 2006). Implementation is not an automatic process: An active approach towards implementation is needed at all levels which the literature describes as important to 
implementation: at the level of the innovation, the implementer, the organization in which the innovation is implemented and the broader socio-political environment (Fleuren, Wiefferink, \& Paulussen, 2004).

The PRECEDE-PROCEDE model (Green \& Kreuter, 1999) systematically guides the planning process of health promotion programs, by providing a continuous series of steps in planning, implementation, and evaluation. Similarly, Intervention Mapping (IM) is a protocol for developing theory-and evidence-based health promotion interventions (Bartholemew et al., 2011). These protocols stimulate the developers to ensure that implementation issues are anticipated throughout the program development process and that implementation is planned for.

Compared to the attention devoted in scientific literature to randomized controlled trials and effect evaluations of health promotion programs, relatively little research has been conducted on the planned large-scale implementation process of these and other health promotion initiatives (Dusenbury, Brannigan, Falco, \& Hansen, 2003; Han \& Weiss, 2005; Oldenburg, Sallis, Ffrench, \& Owen, 1999). Moreover, a limited number of studies have been reported, describing a systematically developed theory- and evidence-based implementation strategy, targeting each stage of the implementation process and considering the broader environment in which the behavior takes place. Effective implementation strategies nonetheless, are worth looking into as they often lead to maximum effect by ensuring that effective programs are adopted on a large scale, implemented with high levels of fidelity and completeness and continued in the long term (Glasgow, Vogt, \& Boles, 1999; Bessems et al., 2011). Designing interventions based on theory and evidence can protect against Type 3 error: failure to produce effective results due to poor design or implementation (Dobson \& Cook, 1980).

More implementation research is required to address this knowledge gap. Implementation research aims to provide insight in several aspects of implementation processes. It includes research on determinants of implementation, the actual development and evaluation of strategies to enhance implementation and research on the relation between implementation and intervention outcomes (Dusenbury et al., 2003; Han \& Weiss, 2005). The implementation research in this dissertation aims to contribute to this implementation knowledge gap.

\section{Implementation of school-based sexual health education programs}

The school system is an important area for health promotion and disease prevention interventions foryouth. It is an ideal setting for health promotion initiatives as adolescents can easily be reached in large numbers (Hoelscher, Evans, Parcel, \& Kelder, 2002). 
Secondary schools in the Netherlands are the primary setting of the research presented in this dissertation. The World Health Organization, (2009) identified school-based programs as one of the most cost-effective approaches to health and development in general. The effect of these programs on the health of the youth is, besides the quality of the program itself, also dependent on the quality of implementation. The reach and implementation of school-based health promotion programs is, however, not optimal (Bessems, van Assema, de Vries, \& Paulussen, 2014; Peters, Kok, Ten Dam, Buijs, \& Paulussen, 2009; Schutte et al., 2014; Forman et al., 2009). Figure 1 shows the implementation process of school-based programs when program developers do not intervene in the process; only $70 \%$ of the target population is aware of the program, $50 \%$ decide to use it (adoption), 30\% actually use it (implementation), and a small 10\% continues to use the program in the long-run (continuation) (Paulussen, Kok, Schaalma, \& Parcel, 1995; Paulussen, Kok, \& Schaalma, 1994).

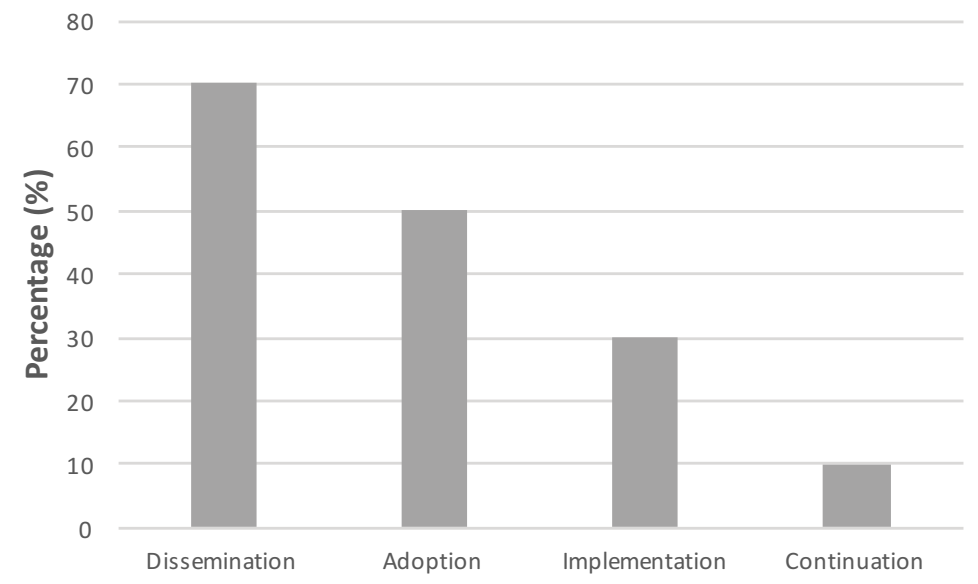

Figure 1. Implementation process of school-based programs without intervention.

A monitoring of school-based interventions in the Netherlands showed that among teachers who bought a program, only $5-10 \%$ implemented it fully in accordance with the ideas of the program designers, resulting in reduced program effectiveness (Hoekstra et al., 2009). Online means of supporting implementation of programs is gaining increased popularity. For example, Hansen, Bishop, \& Bryant (2009) researched the effects of web-based support on implementation of a school-based drug prevention program. They found that the quality of implementation improved, teachers needed less time to deliver the program and that teacher attitudes about the program also 
improved. To date, however, little is known about the use of web-based tools to support the implementation of prevention programs (Bishop, Reiser, Hall, Rein, \& Taylor, 2006; Hansen et al., 2009).

Especially sexual health education programs are poorly implemented in schools (Schaalma et al., 2002; Schaalma, Abraham, Gillmore, \& Kok, 2004 ; Vanwesenbeeck et al., 2015; Durlak \& Dupre, 2008). This is because in most cases, in addition to being a challenging subject to teach, relational and sex education in general is not formally established in schools, it is often not a mandatory element of the school curriculum and providing relational and sex education is not always actively supported by the school board or fellow colleagues (Vanwesenbeeck et al., 2015). Furthermore, if these programs do get implemented, they are frequently modified during implementation by omitting certain program components or activities, using supplementary material or not executing the program as prescribed by program developers (Forman et al., 2009; Berman \& McLaughlin, 1976; Rogers, 2003; Ringwalt et al., 2003; Sy \& Glanz, 2008). Therefore, these programs are not being implemented with sufficient strength and fidelity to produce measurable outcomes (Gottfredson \& Gottfredson, 2002).

There are few published accounts of the process of implementation of interventions once they have been formally adopted by schools, particularly in relation to sex education (Buston, Wight, Hart, \& Scott, 2002). The assumption is often made that adoption at the organizational level will result in adoption and implementation at the teacher level. However, program adoption does not guarantee implementation and teachers' initial attempts will not necessarily result in continued use of the program (Bartholemew et al., 2011). Furthermore, studies concerning sexual health education programs have primarily focused on the dissemination and adoption of these programs, with insufficient attention to their implementation, and continuation (Sy \& Glanz, 2008; Buston et al., 2002). In the Netherlands, the implementation of sexual health promotion programs has been limited to activities aimed at dissemination and adoption and, to program-specific trainings prior to implementation (Kramer, Laumann, \& Brunson, 2000; Paulussen et al., 1995). Less effort is directed at program implementation and continuation. For successful implementation of school-based sex education programs, however, each stage of the implementation process requires attention (Rogers, 2003). Teachers need to be supported in their adoption of a sexual health education program but also need technical and didactic support during implementation to ensure fidelity and completeness as well as support in continued use of the program (Bishop et al., 2006; Durlak \& DuPre, 2008; Rohrbach et al., 2006). The implementation stage in particular is important as the quality with which the program is implemented may influence its 
potential effectiveness. Being key figures in the delivery of school-based sex education programs, investing in the professional development of teachers is necessary to ensure successful implementation (Forman et al., 2009).

\section{Theories and empirical studies explaining the implementation process}

In order to enhance implementation, it is important to first have insight in the factors that could potentially influence the implementation process. Research into determinants of program implementation can help us develop and evaluate implementation strategies (Dusenbury et al., 2003 Han \& Weiss, 2005). Insights can be gained by means of theory and empirical studies on implementation.

The literature describes terms related to implementation differently. On the one hand, the most often used distinction in health promotion is between dissemination, adoption, implementation, and continuation. However, the terms diffusion and dissemination, for example, have been used interchangeably by some in the literature. Diffusion is the unplanned spread of an innovation. This differs from dissemination which refers to the planned systematic efforts designed to make a program or innovation more widely available, with diffusion as a result (Oldenburg \& Parcel, 2002). Rogers (2003), however, sees diffusion as comprising dissemination, adoption, implementation, and continuation. One solution is to distinguish between the planners' behavior (disseminate, diffuse) and the implementers' behavior (program use: adopt, implement, maintain) (Bartholemew et al., 2011). For sake of clarity, in this dissertation, implementation interventions will refer to interventions to increase program use (adoption, implementation, and/or continuation) as is in line with the current implementation movement in the field (Rabin \& Brownson, 2012; Glasgow et al., 2012).

In the field of health promotion, a growing number of models have been developed to describe the factors relating to implementation success of interventions. The implementation process is influenced by factors at the program, the implementer and the organisational level (Humphrey, Lendrum, \& Wigelsworth, 2010; Lendrum, 2010; Durlak \& DuPre, 2008; Lendrum \& Humphrey, 2012). Theories for implementation and related determinants are explained below.

\section{Diffusion of Innovations theory}

The main theory underlying diffusion research is Roger's Diffusion of Innovations Theory (Rogers, 2003). Here, implementation is described as a decision-making process consisting of different stages: (1) awareness of an innovation, through spreading information about the program, potential user receiving, requesting and processing 
information (dissemination), (2) the formation of an intention to buy and use the program (adoption), (3) initial use (implementation), and (4) continued use of the program (continuation) (Rogers, 2003).

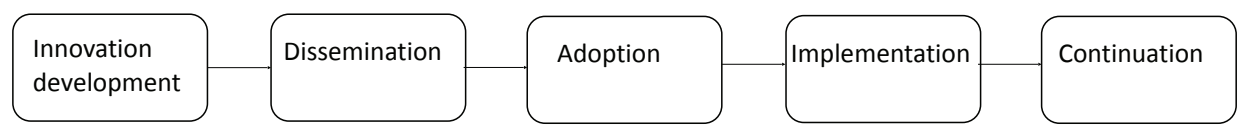

Figure 2. The implementation process (Rogers, 2003).

Innovation development refers to all the decisions and activities that occur from the early stage of an idea through to its development and production (Rogers, 2003). From this stage on, it is important to consider the preferences and feedback of the people who will be using the intervention to reduce barriers to successful implementation (Bartholemew et al., 2011).

Adoption is a decision by the target audience to commit to and initiate a program (Oldenburg \& Parcel, 2002). In this stage, people initially acquire and process information about the innovation and make their decision about using the innovation (behavioral intention) (Rogers, 2003).

Implementation refers to the initial use of the program in practice. It is when an innovation is put into practice by a professional (behavior) (Oldenburg \& Parcel, 2002). A focus on improving self-efficacy and skills of implementers by providing training for example, is required at this stage of behavioral change (Paulussen \& Wiefferink, 2003). The implementation stage has been defined by two dimensions: completeness and fidelity (Durlak, 1998). Completeness refers to the extent of the program being delivered; fidelity is the degree to which the program has been implemented as intended by the developers and as prescribed in the original protocol.

Continuation refers to the ongoing implementation or continued use of the program in practice (Oldenburg \& Parcel, 2002).

This overall process of dissemination, adoption, implementation, and continuation of an innovation is known as the implementation process. Each stage in this implementation process is influenced by different factors at the individual and environmental level. The different stages are, however, dependent on one another and complementary (Forman et al., 2009). This implies that it is necessary to consider all stages of implementation when planning and evaluating the implementation of interventions (Fixens, Naoom, 
Blase, Friedman,\& Wallace, 2005). It also implies that different strategies are needed to enhance each of the stages; dissemination, adoption, implementation, and continuation of an innovation as each stage may involve different actors, different objectives and different factors influencing the actor, and/or the objectives (Rogers, 2003; Forman et al., 2009). According to the diffusion theory, factors that influence adoption decisions and implementation behavior are namely the characteristics of the adopters and attributes of the innovation.

\section{Characteristics of adopters}

Rogers (2003) divides the adopters of an innovation into five different categories, based on the speed at which they tend to accept an innovation. These are innovators, early adopters, early majority, late majority, and laggards (Rogers, 2003). Non-adopters form the last category and never adopt the innovation. The process of adoption can be seen as a normal, bell-shaped curve in Figure 3.

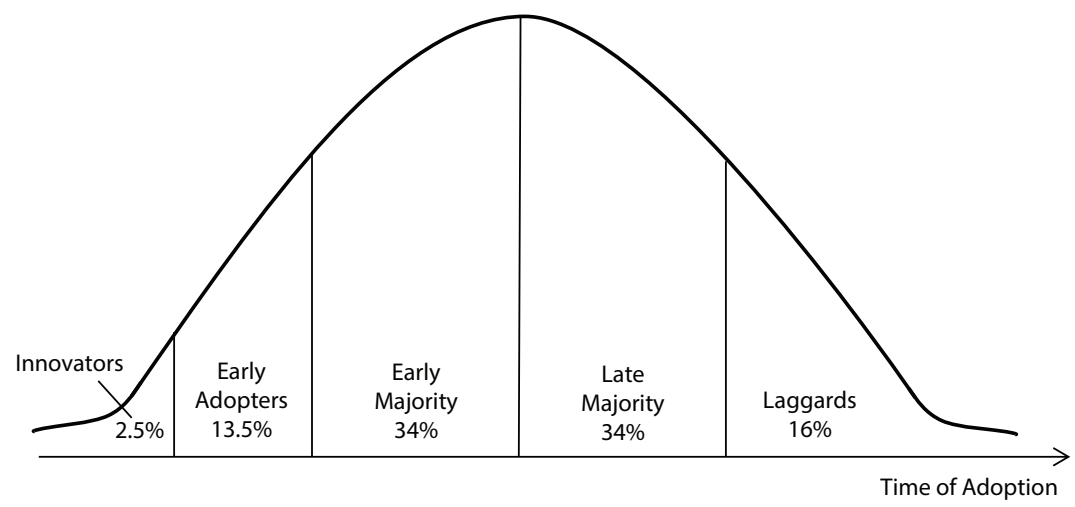

Figure 3. Bell-shaped curve of adoption by individuals (Rogers, 2003).

The identification of such categories provides a basis for designing and implementing intervention strategies aimed at particular groups of individuals and provides understanding for why some innovations are adopted and implemented more quickly than others (Oldenburg \& Parcel, 2002). It should be noted, however, that this categorisation is a generalisation and that the category someone is in differs per innovation. For example, some innovators for one innovation may be laggards in other innovations. Factors outside the individual could also determine the adopter category, such as the absence of a supportive policy to support the adoption decision. 


\section{Attributes of the innovation}

Rogers (1995) identified attributes or characteristics of an innovation that are most likely to affect the speed and extent of the adoption process. These are relative advantage (an innovation demonstrating more advantages than the existing program), compatibility (the fit between the innovation and the intended users), complexity (the ease of the implementation of the program), triability (ability to test the program before deciding to use it) observability (the extent to which the results of an innovation are observable), and reinvention (the degree to which an innovation can be modified by a user during its adoption and implementation (Rogers, 2003).

\section{Other determinants of adoption}

The literature provides studies of factors associated with adoption decisions. Although innovation attributes are expected to determine teachers' adoption decision, their influence will depend on how these attributes match the social and psychological dispositions of teachers (Paulussen, 1994). Therefore, determinants of adoption, other than those suggested by Rogers (1995) should also be considered. In the Netherlands, the study about the adoption and implementation of HIV/AIDS education was guided by the research framework of Paulussen (1994). The cross-sectional survey of 956 Dutch secondary schools showed that teachers' decision making was most strongly related to highly specific adoption-related beliefs (outcome expectations, subjective norms, selfefficacy) (Paulussen, 1994). Adoption was additionally related to generic dispositions (sense of responsibility and sexual morality) and environmental conditions (school policy and frequency of collegial interaction) (Paulussen, 1994). Past experience was also associated with teachers' intentions and related determinants (Paulussen, 1994). Another study by Paulussen et al. (1995) was conducted among 698 Dutch secondary school teachers, addressing the determinants of awareness, knowledge about, and adoption of four nationally disseminated AIDS curricula. It was found that knowledge acquisition was largely dependent on diffusion networks within schools. Adoption was influenced by perceived instrumentality (the extent to which the innovation is believed to meet the practical planning of time and ease once implemented), subjective norms, perceived colleague behavior, and teachers' sexual morality. Similarly, a study by Hoekstra and colleagues (2009) investigated the determinants of teachers' intentions to use school-based programs (adoption). They found that schools that perceived more problems in the area of the prevention program had a higher intention to use the program. Other factors that influenced their intention to use the program were social norms and outcome expectations concerning the prevention program as well as instrumentality. Similar to these findings, Burak (1994) examined factors associated with teachers' intentions to teach HIV/AIDS education (adoption). The framework was 
based on the Theory of Planned Behavior (TPB), where they examined teachers' beliefs, attitudes, subjective norms, and perceived behavioral control regarding AIDS education. These variables explained $64 \%$ of the variance in teachers' intentions (Burak, 1994). These different studies show that adoption-related beliefs, environmental conditions, and general dispositions influence adoption decisions of school-based health education programs among teachers.

\section{Determinants of implementation}

Several studies illustrate factors facilitating or impeding program implementation. Wiefferink and colleagues (2005) examined the effects of a systematically designed innovation strategy on teachers' implementation of a school-based sex education program and its related determinants. Several determinants were measured, including teachers' curriculum related beliefs (attitudes, social influences, self-efficacy), characteristics of the interactive context (school policy, collegial interaction, school's network) and characteristics of the innovation strategy (external consultant, teacher training). Determinants that best predicted teachers' implementation of the schoolbased sex education program were outcome beliefs and perceived instrumentality of the curriculum. Once again, as with adoption, characteristics of the environment and of the teachers are relevant, in addition to characteristics of the innovation strategy, which are specific for implementation. Wiefferink and Paulussen (2003) contribute further to the knowledge of determinants of implementation by stressing the need for more attention to users and the context of the innovation. It is important to understand the environment or context in which the implementation process occurs (Oldenburg \& Parcel, 2002). Users of innovations are not passive. Rather, they are active receivers and processors of new information that they need to apply (Wiefferink \& Paulussen, 2003). Furthermore, teachers who do not feel it is their responsibility to provide sex education or who have no time for providing it are less likely to actually use the innovation. Teachers also often express their need for clear, practical guidelines when using programs, whose availability influences their implementation behavior (Wiefferink \& Paulussen, 2003). The outcomes of an innovation process are strongly influenced by previously existing knowledge, beliefs, and experiences of the users in relation to the innovation (Wiefferink \& Paulussen, 2003). These factors determine whether and how much a teacher makes use of a program or not.

A study in Scotland examined factors that impeded or facilitated the implementation of a teacher-delivered sex education program for youth (13-15 years old) called SHARE (Sexual Health and Relationships: Safe, Happy and Responsible). The extent and quality of implementation was measured as well. Fidelity was aided by intensive teacher training, classroom compatibility, and senior management support. It was hindered by 
competition for curriculum time, brevity of lessons, and teachers'limited experience and ability in the use of role-play (Buston et al., 2002). Studies in other fields of health convey similar findings. A study by Sy and Glanz (2008) examined individual and organizational factors associated with teachers' implementation of a smoking prevention curriculum called Project SPLASH (Smoking Prevention Launch Among Students in Hawaii). The association of the dose of teacher implementation with teacher characteristics, teacher training, and external facilitators and barriers (namely organizational factors, and curriculum attributes) was investigated. Results revealed that implementation dose was related to teachers'skills, self-efficacy, and student enjoyment of the curriculum. Another similar study examined the extent of school-based tobacco prevention curricula and identified factors that enhanced or impeded implementation. Larger organizational size and teacher training were the strongest predictors of curricula implementation and a favorable organizational climate also improved implementation (McCormick, Steckler, \& McLeroy, 1995).

\section{Determinants of continued use}

Even programs that have been successfully implemented in the short-term may not be sustained over time (Oldenburg \& Parcel, 2002). Research suggests that training enables successful implementation by teachers yet it does not guarantee maintenance (Levenson-Gingiss \& Hamilton, 1989). Teachers need to be committed and motivated to continue using a program. It was found that teachers' desire to continue teaching a sexual education course was associated with course-specific attitudes and values, responsibility for student outcomes, comfort using course material, and organizational issues such as class size, accessibility to materials, and scheduling issues (LevensonGingiss \& Hamilton, 1989). School climate, consisting of principal leadership and teacher interactions, was also found to be an important factor in determining sustainability of the Child and Adolescent Trial for Cardiovascular Health (CATCH) program (Parcel et al., 2003).

\section{Theory of Planned Behavior (TPB) and Social Cognitive Theory (SCT)}

Other theories frequently used to explain behavior and to structure the social and psychological dispositions of the target group towards their decision to implement a program are the Theory of Planned Behavior (TPB) (Ajzen, 1988) and the Social Cognitive Theory (SCT) (Bandura, 1986). The TPB claims that intention is the most important predictor of behavior (Ajzen, 1988) under conditions where barriers are not major and skills are adequate. Intention is predicted by attitudes, social influences, and perceived control or self-efficacy. TPB claims that teachers change their behavior when the relevant underlying beliefs change: applying the method of belief selection. SCT 
distinguishes outcome expectations of the behavior, self-efficacy, perceived behavior of others, and environmental factors. Methods for behavioral change which stem from the SCT are: active learning, reinforcement, learning from role-models, and learning by practice and feedback.

\section{Conceptual framework}

The conceptual framework used in this dissertation is based on Paulussen's work (1994) which has frequently been used in the Dutch educational sector (Hoekstra, Beek, Wiefferink, \& Buijs, 2007). Similar to the work of Paulussen and colleagues, (1995) our framework integrates the Diffusion Theory with the TPB and SCT, relating it specifically to the Long Live Love curriculum. In this framework, the determinants that influence each stage in the implementation process were placed into four categories: (1) curriculumrelated beliefs, (2) interactive context, (3) information sources, and (4) demographic variables of teachers and schools. The factors within these different categories influence each stage in the implementation process to a different degree.

Three clusters of curriculum-related beliefs-attitudinal, normative, and self-efficacy beliefs - are assumed to affect curriculum adoption, implementation, and continuation most directly. These curriculum-related beliefs in turn may be influenced by the other three categories. The interactive context, demographic variables, and information source are believed to influence teacher's adoption decision and implementation, and continuation behavior, either directly or indirectly.

The demographic variables of teachers (such as gender, age, years of teaching experience) and schools (such as size, denomination) may influence adoption and implementation of school programs.

The interactive context consists of environmental and organisational conditions associated with the acceptance of new classroom practices such as; a schools' formal sexual education policy, governing body support (context), the frequency of collegial interaction about sexual instruction, the extent of use of sexual education curriculum by colleagues (descriptive norm), the students' response to the curriculum and the social-political context. These factors can influence teacher's adoption decision and implementation, and continuation behavior.

The information source refers to the implementation strategy or support from external consultants, namely the municipal health services (MHS), web-based coaching and additional training for teachers to implement the innovation. Attending teacher training, 
receiving web-based coaching, and external consultation can facilitate adoption and implementation by enhancing teachers' skills and self-efficacy with regard to sex education.

\section{Organization of public health and the school system in the Netherlands}

In the Netherlands, the Ministry of Health and the Ministry of Education are responsible on a national level for financing health promoting institutions, schools, and municipalities and for creating policies on a national level. STI Aids Netherlands is financed by the Ministry of Health. The provincial public health services are responsible for different selected regions of the country. Each province in the Netherlands has a regional Municipal Health Service (MHS) that is by law responsible for enhancing public health. The MHS is responsible for regional health promotion and supports schools in delivering health education programs. They focus on several fields of health, of which sexual health is one aspect. Each provincial municipality decides which health topics are essential to focus on in their regions and which ones to prioritize and then provide the funding to the MHS. Each MHS then needs to decide which programs and corresponding implementation promotion tasks they want to focus on and execute. In 2011, right before the start of this dissertation project, there were $28 \mathrm{MHS}$ in the country. The MHS often act as intermediaries who form the linkage between program developers and schools. The 'Health Promoting School' (Gezonde School Methode) approach is used by numerous MHS' in which the MHS, together with schools determine what health topics will be prioritized and then incorporate these activities in the school health policy (Boot, van Assema, Hesdahl, Leurs, \& de Vries, 2010). This is a more demand-oriented approach in which schools determine which health topics to prioritize. Other MHS' work with a more supply-oriented approach in which they actively approach schools with particular health promotion programs and stimulate schools to use them.

Secondary schools in the Netherlands are professional autonomous organisations where teachers themselves, rather than the hierarchal top of the organisation, decide what to include in the education they provide. Teachers are thus autonomous in their selection and use of health education programs, without interference of external authority in the details of the curriculum in schools (Paulussen et al., 2007). Although decisions to use programs in schools are typically made at the administrative level, teachers are the primary agents of school-based prevention efforts. Their support, motivation, and commitment is crucial to implementation success (Hunter, Elias, \& Norris, 2001). In the Netherlands, teachers are the ones who decide to use a school-based program in their classroom (adoption), deliver the program to students (implementation), and continue to do so in the long run (continuation) (Paulussen et al., 1995). These are mostly teachers 
of biology, social studies, religious education and health education (Paulussen et al., 1995). Many evidence-based programs consider the implementers of the programs, such as teachers, as "core" to the success of the program effects (Coyle, Firpo-Tiplett, Taylor, \& Rex, 2011). Teachers thus play a central role in effective implementation of school-based programs.

Although sexual health education has not yet fully been integrated into school policy, since December 2012, a new law stipulating the key targets of Dutch secondary education was enacted, making two core educational objectives in the field of sexual health mandatory. These are: sexuality and sexual diversity. This obliges school directors to reserve budgets yet there are no contours defining how these core targets should be achieved (Bron, Loenen, Haverkamp, \& van Vliet, 2015). The schools in which teachers work are expected to provide a supportive environment for the implementation of sexual education programs but there is no rule stating that the program used for sex education has to be evidence-based. See Figure 4 for an overview of all parties involved.

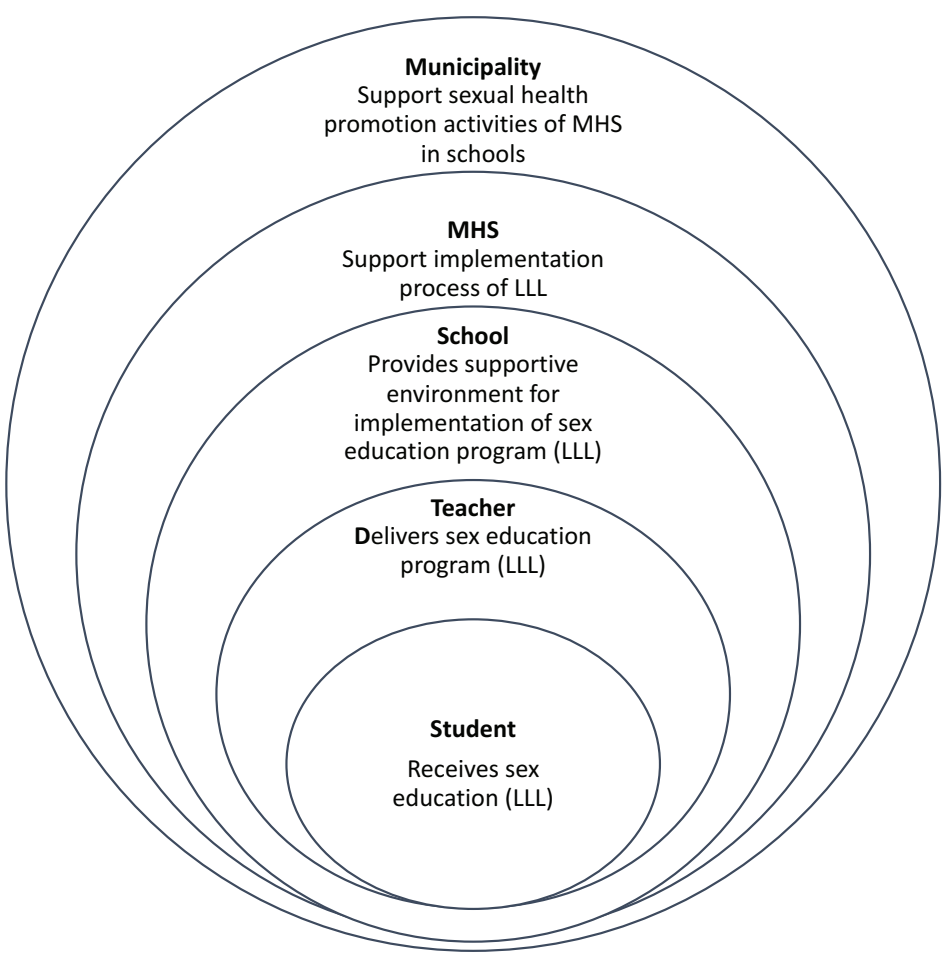

Figure 4. Parties involved in implementation process of LLL. 


\section{Long Live Love and its history}

Previous research and evaluations have shown school-based sex education programs to be effective in changing behaviors (Fisher \& Fisher, 1992; Jemmott \& Jemmott, 2000; Kirby, Obasi, \& Laris, 2006; Kirby, 2001; Kirby, 2002; Saito, 1998). The Netherlands is renowned for the quality of its sexual health promotion for the youth (de Graaf, Meijer, Poelman, Vanwesenbeeck, 2005; Kocken et al., 2007).

Long Live Love (LLL) is by far the most successful, effective, evidence-based program in the field of school-based sex education in the Netherlands (Fulpen et al., 2002; Schaalma et al., 1996). Long Live Love is a sexual health education program for secondary school students aged 13-15. The first version of LLL was developed 26 years ago and was shown to produce desirable student learning outcomes, when correctly applied (Schaalma et al., 1996). Since then the LLL curriculum has been revised four times. The fourth revised generation of the program was recently developed and launched in 2012. LLL was developed by STI Aids Netherlands and the revision and implementation project was financed by the Netherlands Organization for Health Research and Development (ZonMw).

Long Live Love was developed systematically using the Intervention Mapping planning tool to ensure a theory and evidence based intervention. The aim was to develop an effective program that is compatible with the teaching practice and fits the perceptions and reality of Dutch students. LLL was developed while anticipating implementation and in close collaboration with teachers, students, and MHS professionals, incorporating their needs and preferences. This involvement and collaboration is the first step towards overcoming implementation barriers and promoting optimal implementation as it creates a sense of ownership for the program and ensures its compatibility with the skills and context of the intermediaries. This subsequently increases the probability of the program being used (Greenberg, 2004).

The newly revised Long Live Love aims to assist youngsters in the healthy development of their emerging sexuality. It not only addresses knowledge but is also aimed at attitude, skills and behavior change. LLL is a teaching pack that consists of a student magazine, a DVD or online films, digital lessons, and a teacher manual. See Figure 5. The teacher manual includes clear indications of the core activities per lesson. 

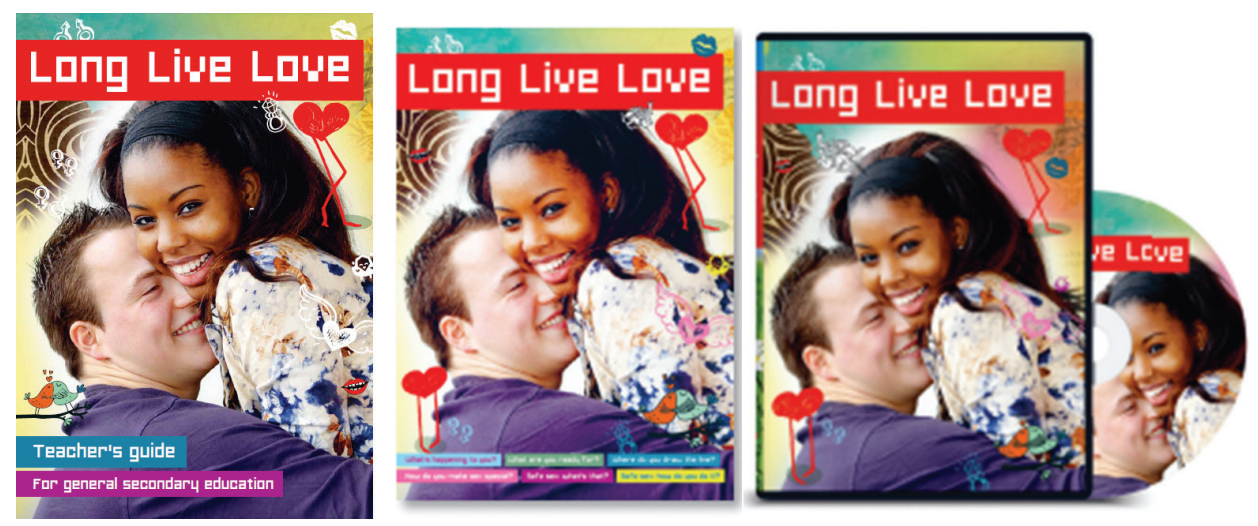

Figure 5. New LLL curriculum (made available in 2012).

\section{The six les5uns}

The teaching pack consists of six lessons.

Each lessons is made for one lesson period of 50 minutes.

\section{What's happening tᄆ 4 미?}

LESSON 1 Puberty, relationships, homosexuality, influence of parents and friends on beginning a relationship.

\section{What are 니 ready for?}

LESSON 2 Chatting up and going out with someone, ending a relationship, dealing with a broken heart, types of intimate physical contact.

\section{Where do 4ㅁ draw the line?}

LESSON 3 Discovering what you want and where you draw the line, finding out how the other person feels about that, using the internet safely, undesirable experiences, groomers.

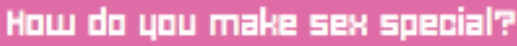

LESSON 4 The first time, having sex only when you are ready, coping with problems regarding sex and getting help for your questions and problems.

\section{5afe 5ex: what's that?}

LESSON 5 Preventing pregnancy, preventing sti's, safe and unsafe sexual behaviour and what to do after unsafe sex.

\section{5afe 5eK: hㅁ da 니 da it?}

LESSON 6 Safe condom use \& contraceptive use, talking about it and coping with resistance to having safe sex.

Figure 6. LLL lessons - from information leaflet LLL. 
LLL compromises six lessons administered mainly by teachers of care and biology. See Figure 6. LLL focuses on the biological and relational aspects of sexuality. It not only targets unwanted teen pregnancies and STI's but also assertiveness, communicating, respecting and accepting boundaries, HIV/STI testing, managing relationships, and dealing with sex on the internet.

Long Live Love uses a positive approach to sexuality, taking into consideration gender-, cultural- and sexual diversity and differences in experiences with sexuality. LLL needs to be implemented structurally, delivering each lesson according to the teacher manual and using all program components. The program is flexible enough to use in diverse classrooms.

\section{Past Implementation experiences of Long Live Love}

A successful implementation of LLL should ultimately lead to young people being better prepared, motivated, able to practice safe sex, and have mutual, pleasant relations. Previous versions of LLL were found to be effective due to systematic program development and by anticipating and planning for implementation (Bakker et al., 2002; Schaalma et al., 1996). An implementation strategy was developed in 2002 to promote adoption, implementation and continuation of LLL on a national level (Wiefferink et al., 2005). This was the SLIM project (Systematische Landelijke Implementatie/ Systematic National Implementation) aimed at promoting large-scale and adequate use of LLL. A national project team recruited 15 municipal health services (MHS) to help them with the implementation. The MHS had an active, supportive and stimulating role in the dissemination and implementation of LLL by teachers in schools. The MHS professionals provided teachers with training before the implementation of the program and also provided assistance during the implementation. Their task was facilitated by the national project team by means of training and follow-up support (Wiefferink et al., 2005). MHS professionals used different strategies to address the differing needs of the teachers: mass media was used as well as interpersonal contact to encourage adoption of the program among the less innovative teachers (Wiefferink et al., 2002).

Despite this systematic approach, the implementation of previous LLL was nevertheless still not optimal; certain program activities were still being omitted by teachers, the program was not always implemented as prescribed by the developers, and teachers tended to stop using the program after one or more implementations (Bakker et al, 2002; Wiefferink et al., 2005). There is thus room for improving the quality of the implementation of the new LLL by secondary school teachers. 


\section{Development of the new Long Live Love implementation strategy}

This project can be considered a continuation of the SLIM project that was used for the previous versions of LLL, using new approaches and strategies. The existing implementation strategy ultimately needed to be optimized. The outdated Long Live Love program was being revised and a new, upgraded, and compatible implementation strategy was required for this new program. In order to have a public health impact, the revised LLL needed to be adopted, implemented, and sustained on a large scale by teachers (Durlak \& DuPre, 2008; Glasgow et al., 1999).

Additionally, the economic crisis that was prevalent at the time the new LLL program was launched, impacted the MHS, resulting in them having to play a different and more limited role in supporting school-based health promotion. The MHS sometimes lacked the capacity, didactic expertise, and skills to provide long-term support for adequate implementation. Alternative forms of support were required. Better support for teachers was recommended to improve program implementation. Relying on mass media communication and one-time teacher workshops was inadequate to achieve widespread diffusion and behavioral maintenance (Schaalma et al., 1996). It was important instead to provide teachers with more personal assistance and ongoing consultation once the program had begun (Durlak \& DuPre, 2008). Paulussen and colleagues (1995) already highlighted the importance of engaging personally prior to and during the implementation of a curriculum by way of training and technical assistance to ensure success.

\section{Intervention Mapping protocol}

In order to achieve successful implementation of the future LLL program and other school-based (sexual) health promotion programs, an up-to-date evaluation of factors influencing adoption and implementation was necessary. In this project, we planned to evaluate the adoption and implementation of the previous version of LLL and subsequently systematically developed a national implementation strategy for the optimal adoption, implementation, and continuation of this revised sexual health promoting intervention by teachers in secondary schools. This was done using the Intervention Mapping protocol (IM).

The Intervention Mapping (IM) protocol forms the foundation of this entire project and is the systematic means by which the implementation strategy has been planned for and developed. Interventions have the highest chance of being successful if they are systematically planned, that is to say; when every step in the development cycle is taken 
based on empirical research, theoretical insights and close collaboration with the target group and intermediaries, and when evaluations are conducted (Bartholemew et al., 2011).

Intervention Mapping (IM) describes the development of an intervention in six steps (Kok, Schaalma, Ruiter, Brug, \& van Empelen, 2004; Bartholemew et al., 2016; Schaalma, \& Kok, 2009): (1) analyse the problem, (2) formulate specific goals for changes in behavior and environment, (3) select theoretical methods for change and practical applications of these, (4) develop and test the program, (5) anticipate for implementation and (6) anticipate for evaluation. IM urges program developers to go through all IM steps in close collaboration with the target group and stakeholders (teachers, MHS), to take the environment and its agents into consideration and take decisions based on empirical findings and theoretical insights to increase the chance of effectiveness.

The Long Live Love program was developed using IM, following steps 1 to 4 . The fifth step of IM focuses on planning for the implementation of the health promotion program and this entire dissertation is based on this step. In step 5 of IM, the entire IM process that is followed for intervention development is followed again to develop an implementation strategy for that intervention, now targeting individuals (teachers) and environmental agents (MHS professionals), other than the target group of the intervention (youngsters). A top-down and bottom-up approach was used to develop this implementation strategy, with IM guiding the theory- and evidence-based systematic intervention development and input and involvement of the stakeholders assisting in creating a sense of ownership and compatibility of the program to their reality. A combination of research theory and practice is thus necessary to develop an implementation strategy.

The success and effectiveness of LLL is expected to not only be dependent on the quality of the program itself, but partly on the extent and quality of its implementation by teachers as well (Bartholomew et al., 2006; Durlak \& DuPre, 2008; Norton, Amico, Cornman, Fisher, \& Fisher, 2009; Zins, Elias, Greenberg, \& Pruett, 2000). Thus, on the one hand, the LLL intervention is a tool for delivering sex education and is compatible with the teaching practices and skills of teachers. On the other hand, the implementation strategy is meant to support teachers in delivering the LLL lessons with the best quality possible (i.e. high levels of completeness and fidelity), to ultimately achieve effective results in the sexual health of students. 


\section{Aim and outline of this dissertation}

The aim of this dissertation is to systematically plan, develop and evaluate an implementation intervention to promote the dissemination and adoption of the revised Dutch school-based sexual health program Long Live Love, by teachers in secondary schools, to improve the quality and extent of its implementation and to sustain its use over time, thereby contributing to the success and effects of LLL on youngsters. To develop the implementation strategy for the revised LLL, all the IM steps were followed; first needs assessment studies focusing on teachers and MHS professionals were planned, followed by formulating program objectives for teachers and MHS professionals, selecting methods and strategies, developing an implementation promoting intervention, and concluding with program evaluation.

The new implementation strategy addressed the teachers at the individual level, to stimulate their adoption, implementation and continuation of LLL. It also addressed health promotion professionals from the MHS at the environmental level, who were supported by program developers in their implementation-promotion activities of LLL in schools. Different tools were developed to promote each stage of the implementation process. Each tool could be enacted during the stage of implementation at which it was most relevant. All tools together formed the implementation strategy.

Although all the stages of the implementation process were addressed with the implementation strategy, the focus of this dissertation will mainly be on the e-coaching intervention as an web-based innovative component of the implementation strategy. This is a website supporting teachers in the implementation of LLL, focusing on enhancing two dimensions of implementation, namely completeness and fidelity. Focus on the implementation stage in particular is important as the quality with which a program is implemented may influence its potential effectiveness (Durlak, 1998; (Domitrovich et al., 2008). E-coaching's adoption, use and effects on the implementation behavior of teachers were analysed. Insight is given into the implementation of LLL by teachers, the support provided by the MHS and the effects of the implementation strategy, specifically the e-coaching website, on the implementation behavior of teachers.

\section{Chapters}

Chapter 2: In this quantitative study, teacher's classroom implementation of the previous LLL is evaluated, and the determinants of the implementation process are examined among secondary school teachers $(\mathrm{N}=130)$. This gave insight into facilitating and hindering factors of each stage in the implementation process. This input was used to develop the implementation strategy for the revised LLL. Understanding the 
factors that influence each of these implementation stages is crucial in explaining and improving the effectiveness and implementation of school-based sex-education programs specifically or school-based interventions in general.

Chapter 3: In this research study, interviews $(\mathrm{N}=19)$ and surveys $(\mathrm{N}=26)$ were used to provide insight into factors influencing the intention and ability of the MHS for (continued) implementation promotion activities of the previous and revised LLL program. The research focuses on the role of the MHS, from the perspective of the MHS professional, and their perceptions of barriers and facilitating factors in supporting the implementation process of the LLL program.

Chapter 4: This paper describes the systematic development of e-coaching using the Intervention Mapping protocol. Our basic assumption is that teachers are key players in program implementation and require support for optimal program implementation. E-coaching or 'teachinglove.nl'/'lesgevenindeliefd.nl' is an online coaching website to support teachers in the implementation of LLL with completeness and fidelity. This is the most innovative component of the implementation strategy.

Chapter 5: A randomized controlled trial (RCT) effect evaluation of the web-based coaching intervention is described and its effects on teacher implementation behavior and determinants are reported and explained. A total of 43 schools with 83 teachers participated in the study. In the follow-up 38 schools participated; 23 in the e-coaching condition with 41 teachers, 15 in the control condition with 26 teachers.

Chapter 6: A process evaluation was conducted, including surveys $(\mathrm{N}=67)$ and additional interviews $(\mathrm{N}=20)$ among teachers who had access to the e-coach, in which appreciation and (motives for) use of the e-coach were investigated. In addition to providing possible explanations for the outcomes of the effect evaluation, it provides insights into potential improvement of the e-coaching website.

Chapter 7: The dissertation ends with a general discussion, including implications and recommendations for research and the public health field. 


\section{CHAPTER 2}

\section{Long Live Love: The implementation}

\section{of a school-based sex education}

program in the Netherlands

This chapter is published as:

Schutte, L., Meertens, R., Mevissen. F.E.F., Schaalma, H., Meijer, S., Kok, G. (2014). Long Live Love:The implementation of a school-based sex-education program in the Netherlands. Health Education Research; 29(4):583-597. DOI:10.1093/her/cyu021 


\section{ABSTRACT}

Implementation of health education programs is often inadequately considered or not considered at all in planning, developing and evaluating interventions. With the focus being predominantly on the adoption stage, little is known about the factors influencing the implementation and continuation stages of the implementation process. This study contributes to the understanding of factors that promote or impede each stage of the implementation process in the school setting using the sex education program Long Live Love (LLL) as an example. A survey integrating different implementation-related concepts was completed by 130 teachers. Results showed that teacher curriculumrelated beliefs were associated with all stages in the implementation process. Whereas adoption of LLL was predominantly related to teacher curriculum-related beliefs, implementation completeness and fidelity and continued use of LLL were also enhanced by contextual factors, namely teacher training and interactive context variables (school policy, governing body support, and student response) respectively. The results of this study can be used to optimize the adoption, implementation and continuation of school-based (sexual) health promotion programs. 


\section{INTRODUCTION}

School-based sex education programs are the primary means by which adolescents in the Netherlands receive information and skills related to safe sex, communication about sex and managing relationships (Kirby, 2002). A multitude of interventions have been developed globally for sex education of youngsters in school (Stephenson et al., 2004; Visser \& van Bilsen, 1994). Although sometimes proven effective, other interventions show only short-term or no effects (Kirby, 2002; Kirby et al., 2006). Besides due to an ineffective content, these inconsistent findings may also be explained by inadequate implementation. Not being completely or correctly implemented can greatly undermine the effectiveness of an intervention (Bartholemew et al., 2011). Indeed, the impact of school-based health education programs is often attenuated by inadequate teacher implementation (Resnicow et al., 1998). Implementation is thus a crucial aspect of planning and delivering successful health education programs yet it receives insufficient attention (Bartholomew et al., 2011; Green \& Kreuter, 1999; Oldenburg \& Parcel, 2002).

There are few published accounts of the process of implementation of interventions once they have been formally adopted by schools, particularly in relation to sex education. Little is known about if, how or how well the material is covered. Research conducted in the implementation field has tended to focus primarily on the adoption stage (Buston et al., 2002). Considerably less effort has been devoted to determining whether and how new programs are actually used in classrooms after being adopted. The assumption is often made that adoption at the organizational level will result in adoption and implementation at the teacher level. However, program adoption does not guarantee implementation and teachers' initial attempts will not necessarily result in continued use of the program (Bartholemew et al., 2011). Understanding the factors that influence each of these stages is therefore crucial in explaining and improving the effectiveness of school-based sex-education programs specifically or school-based interventions in general.

This study attempts to fill that gap by focusing on all the stages in the implementation process, providing a holistic explanation of the adoption and implementation behavior of teachers in the school context. The present study addresses the promoting and inhibiting factors of teacher adoption, implementation, and continuation of a Dutch school-based sexual education program called Long Live Love. The Long Live Love (LLL) program is one of the most successful, evidence-based programs in the field of school-based sex education in the Netherlands targeted at adolescents (13 to 15 years) in secondary vocational schools. The effectiveness of previous versions of this program has been largely accredited to the quality and extent of its implementation (Bakker et al., 
2002; Schaalma et al., 1996). In the current study, teacher's classroom implementation of Long Live Love is evaluated and the determinants of the implementation process are examined.

\section{Long Live Love}

In the Dutch education system, schools and teachers are autonomous in their selection and use of health education programs, without the interference of external authority. Sex education is also provided on a voluntary basis, mostly by biology teachers (Paulussen et al., 1995). Long Live Love is the most widely-used evidence-based teacher-delivered program for sexual education in the Netherlands, proving it to be a worthwhile intervention (Schaalma et al., 1996). Over 50\% of vocational schools have bought the program (Poelman, 2008). The first version of Long Live Love was developed 24 years ago and was shown to produce desirable student learning outcomes, when correctly applied (Schaalma et al, 1996). Since then the LLL curriculum has been revised three times and another revision is in progress. The last evaluation, however, was done in 2002 (Schaalma et al., 2002). In order to improve successful implementation of the future Long Live Love curriculum and other school-based (sexual) health promotion programs, an up-to-date evaluation of factors influencing adoption and implementation is necessary.

Long Live Love is a relational and sexual education program composed of 26 learning activities divided over six lessons of 50 minutes each. 22 of these activities are core and 4 are optional. LLL is designed to provide students with communication and negotiation skills to enable safe sex practices. It compromises a teacher's manual, a student magazine, and DVD. The main objective is the prevention of STD's/HIV and unplanned pregnancy (Poelman, 2008). The presented framework will guide the evaluation of the LLL program.

\section{RESEARCH FRAMEWORK}

The general outline of the research framework for this study was derived from an integration of Roger's Diffusion of Innovations theory, the Theory of Planned Behavior (TPB), Social Cognitive Theory (SCT) and from previous research on innovation in AIDS education in Dutch schools (Paulussen, 1994; Paulussen et al., 1995).

Roger's Diffusion Theory (1995) describes implementation as a decision-making process consisting of different stages; (1) awareness of an innovation, through spreading information about the program, potential user receiving, requesting, and processing 
information (dissemination), (2) the formation of an intention to buy and use the program (adoption), (3) initial use (implementation) and, (4) continued use of the program (continuation) (Bartholemew et al., 2011).

The TPB claims that intention is the most important predictor of behavior (Ajzen, 1991). According to Paulussen et al., (1994), intention and behavior in this context can be considered synonymous for adoption and implementation, respectively. Adoption thus refers to the intention of teachers to use the curriculum during sexual education. Only once a program has been adopted can it be implemented. Implementation of the curriculum refers to performance of the behavior, the actual use of the program. The implementation stage has been defined by two dimensions: quality and quantity. Quantity or extent/completeness is how much of the curriculum is taught; quality or fidelity is the measure in which the program has been implemented as intended by the developers. Different factors influence each stage in the implementation process.

The framework used in our study included the adoption of a revised version of LLL and the implementation and the continuation stages of the current version of LLL. Adoption of the revised LLL program was focused on instead of the current $L L L$ program because all respondents included in the study have already adopted the current LLL program and there was more interest in inquiring what factors need to be taken into consideration to promote adoption of the revised LLL. Furthermore, past experience with programs has been found to influence future use of it (Paulussen et al., 1995; Han \& Weiss, 2005; Johnson et al., 2003; Levenson-Gingiss \& Hamilton, 1989).

The framework is presented in Figure 1. The determinants that influence each of these stages were investigated. These determinants have been placed into four categories; (1) curriculum related beliefs, (2) interactive context, (3) information sources and (4) demographic variables. Three clusters of curriculum related beliefs - attitudinal, normative, and self-efficacy beliefs - were assumed to affect curriculum adoption, implementation and continuation most directly. These curriculum related beliefs in turn may be influenced by the other 3 categories. The interactive context and information source are believed to influence teacher's adoption decision and implementation, and continuation behavior, either directly or indirectly. 


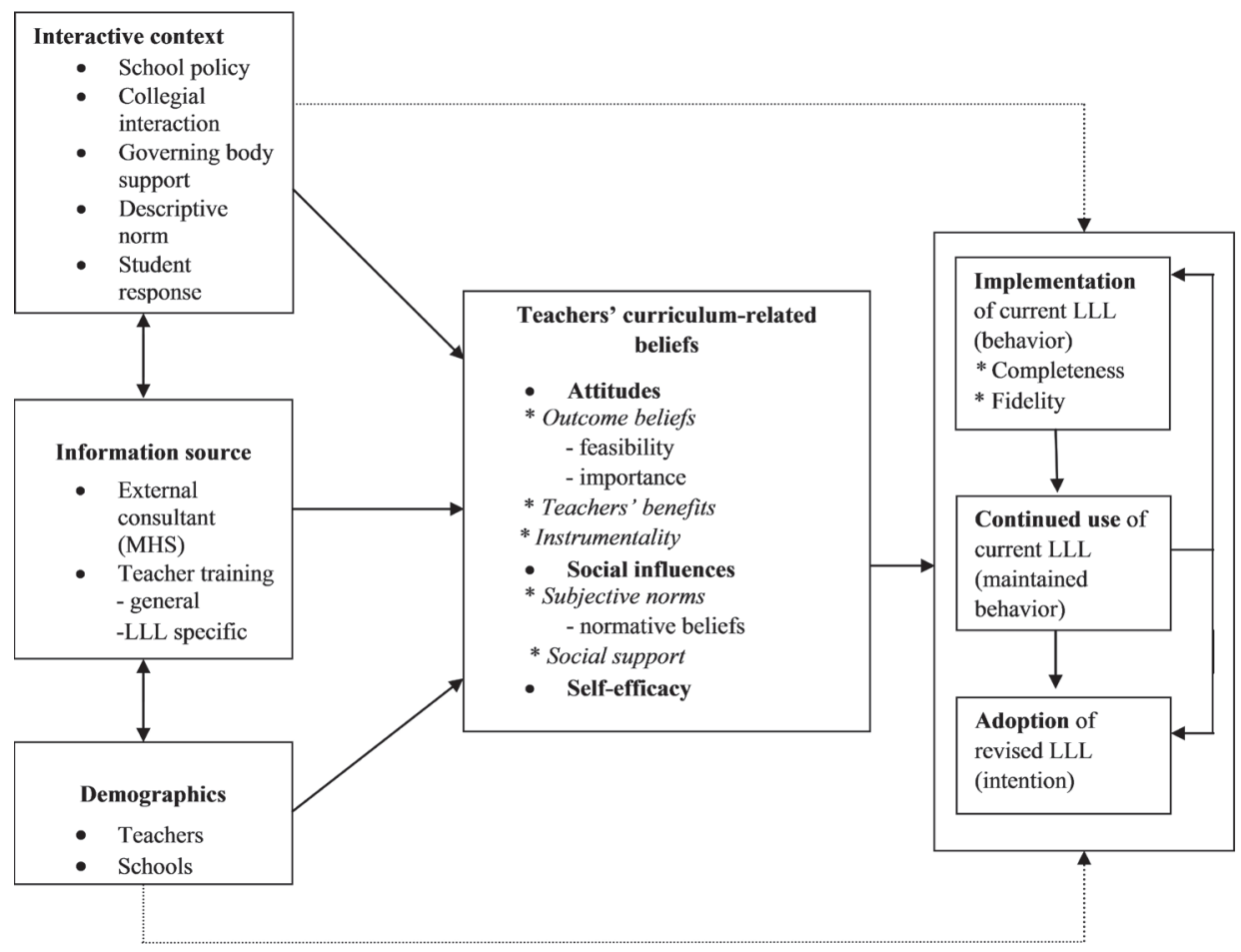

Figure 1. Framework for investigating the correlates of teachers' LLL implementation-decision process, adapted from Paulussen et al., 1994.

\section{Teacher's curriculum-related beliefs}

Teacher's classroom implementation is best explained by their curriculum-related beliefs which include their attitudinal, normative, and self-efficacy beliefs towards that particular innovation (Paulussen et al., 1994; Sarama, Clements, \& Hery, 1998; Wiefferink et al., 2005). Perceived importance and feasibility of student learning outcomes (outcome beliefs) are assumed to capture teachers' attitudes toward classroom sexual education (Paulussen et al., 1994). Also under attitude are teachers' benefits (the personal advantages that the curriculum could have for the teacher) and instrumentality (practicality of program use in practice related to how acceptable the intervention is from a practical point of view) (Paulussen et al., 1995).

Important individuals might provide normative standards for teachers' decision to implement a new program (Paulussen et al., 1994). Subjective norms are conceptualized as the attributed normative beliefs of important social referents, such as students, colleagues and parents (Ajzen, 1991). Social support involves the affective and/or 
instrumental support expected of social referents in the teachers' environment, namely the governing body, colleagues teaching the same and different subjects and the parent association (Wiefferink et al., 2005). Self-efficacy refers to one's perceived ability to perform a particular behavior, in this case, teachers' ability to implement the LLL curriculum in their classrooms with confidence (Bandura, 1986). Self-efficacy is often found to be a strong predictor of the implementation of curriculum innovations, especially for sex education (Burak, 1994; Paulussen et al., 1995).

Teachers are expected to deliver more of the program (completeness) with higher integrity (fidelity) if they have a more positive judgment of the curriculum (Is it beneficial to use LLL? Can I integrate it easily in my lessons?), if they think that others believe they should use the curriculum (what do my colleagues think?) and the more they are able and skilled to work with the curriculum (can I work with the class material?). Teachers' experience with the program in turn will influence their intention to use LLL again (continuation) and/or to use the revised LLL (adoption). Their intention is expected to be higher if their attitude towards the program is positive, if they believe other teachers also intend to use the program, and if they have the skill and ability to work with the program (Wiefferink et al., 2005).

In the Netherlands, a study about the adoption and implementation of HIV/AIDS education among 956 Dutch secondary schools showed that teacher's decision making was most strongly related to highly specific adoption-related beliefs (outcome expectations, subjective norms, self-efficacy) (Paulussen, 1994). Similarly, Hoekstra et al. (2009) investigated teacher's intentions to use school-based health education programs on self-development and anti-bullying (adoption). Factors that influenced teachers' intention to use the programs were social norms and outcome expectations concerning the prevention program as well as instrumentality.

\section{Interactive context}

The interactive context consists of environmental and organizational conditions in which teachers have to implement sexual education in their school. The interactive context refers to a schools' formal sexual education policy, governing body support (context), the frequency of collegial interaction about sexual instruction, the extent of use of sexual education curriculum by colleagues (descriptive norm), and the students' response to the curriculum. Teachers have to teach in collaboration with their colleagues and within the boundaries set by the policy of their school (Wiefferink et al., 2005). Curriculum implementation is thus assumed to be facilitated by a clearly stated school policy in the schoolwork plan or their own curriculum work plan (Campbell \& Lubben, 2003), and by interactions of teachers with the school management and their 
colleagues about instructional matters (Smylie, 1988). In case conditions of frequent collegial interaction are not present at a school, perceived behavior of colleagues may operate as a descriptive norm for strengthening teachers' own implementation decision (Grube, Morgan, \& McGree, 1986). Students' reactions to the curriculum are expected to influence the extent of implementation, with positive reactions resulting in more of the program being used (Sy \& Glanz, 2008).

\section{Information source}

The information source refers to support from external consultants, namely the municipal health services (MHS) and additional training for teachers to implement the innovation. In the Netherlands, the MHS is responsible for regional health promotion and supports schools in delivering health education programs. Attending teacher training and receiving external consultation can facilitate adoption and implementation by enhancing teachers' skills and self-efficacy with regard to sexual education (Paulussen, 1994). It has been shown that the provision of pre-implementation training increases the likelihood that teachers will implement the curriculum fully and with integrity (Rohrbach, Graham, \& Hansen, 1993). Previous studies found that implementation dose was associated with having received training on that specific curriculum (Johnson et al., 2003; Parcel, Ross, \& Lavin, 1991; Perry-Casler, Price, Telljohann, \& Chesney, 1997; Rohrbach, et al., 1993). A study examining the extent of school-based tobacco prevention curricula found that trained teachers were more likely to implement and to implement more of the curriculum than untrained teachers (McCormick et al., 1995).

\section{Demographic variables}

Several demographic characteristics of both teachers and their schools may influence adoption and implementation of Long Live Love such as teachers' gender, age, years of experience with LLL, schools' denomination (Catholic, Protestant, Public) and class composition.

\section{Other variables}

To complement the determinant study, several constructs that are not represented in the framework were added, namely what LLL program components teachers use in their lessons (teacher's manual, student magazine, DVD), the hours they spend on teaching the LLL program and the extent of familiarity with the program prior to using it, as this could influence implementation behavior (Durlak \& DuPre, 2008). Additionally, open-ended questions were included to reveal teachers' reasons for their intentions to continue using the current Long Live Love program or to adopt the new LLL program. 


\section{METHOD}

\section{Participants \& recruitment}

A list from the educational publisher of teachers who have ordered the Long Live Love program since 2006 was used to recruit teachers. A questionnaire was sent by post to a total of 610 teachers who are working or have worked with LLL. A total of 130 teachers from 110 schools completed the questionnaire, a response rate of $21.3 \%$. Twenty-five questionnaires were returned due to incorrect addresses or teachers no longer working in those schools. Non-responders got a reminder by post, e-mail and eventually by telephone and were given two more weeks to fill out the questionnaire. No official nonresponse research was conducted due to a shortage of time and schools closing for the summer holiday.

The participating schools were well distributed over the different regions of the Netherlands. Half the schools $(50.8 \%, \mathrm{~N}=66)$ had no religious background and a small school size ( $\leq 500$ students) with $58 \%(\mathrm{~N}=73)$ of teachers having a class compositions of predominantly native students. Of the participating teachers 104 were female (80\%). The mean age was 44 years $(S D=10.4)$. Years of teaching experience ranged from 1 to 42 years $(M=22 ; S D=10.3)$, while years of experience teaching sexual education ranged from 1 to $34(M=8 ; S D=6.7)$, and years of experience with LLL ranged from 1 to $10(M=$ $4 ; \mathrm{SD}=2.51$ ). About $94 \%$ were teachers of biology and healthcare.

\section{Procedure}

Across-sectional study of teachers who provide sexual education at secondary vocational schools in the Netherlands, using or having used the Long Live Love program, was conducted. Teachers received an envelope containing an official letter with instructions for filling out the questionnaire and a return-envelope in which they could send back the filled-out questionnaire, free of charge. A ten-euro gift voucher as well as the option to participate free-of-charge in the sex education workshops ('Youngsters, sex and Islam' and 'Youngsters, sex and internet'), were offered as reward. Teachers were given two weeks to complete and send back the questionnaire. Anonymity and confidentiality were preserved throughout the study.

\section{Measures}

The items included in the questionnaire were based on the scales used by Paulussen (1994) and Wiefferink et al (2005). Implementation and continuation refer to the current LLL program whereas adoption refers to teacher's intention to use the new LLL that is currently under development. 


\section{Dependent variables}

Completeness or extent of use of Long Live Love was expressed as the percentage of the program (i.e. learning activities) being implemented. For each of the 22 core learning activities in LLL, teachers were asked if they had completed that activity. The completeness of implementation of the other four activities was not included in the analyses as these were optional. In the end, completeness was calculated for each teacher by adding up all the activities they completed per lesson, dividing them by the total number of activities (maximum 22) and multiplying them by 100 .

Fidelity or quality of use was measured by asking teachers to indicate, per lesson, how well they followed the instructions in the teacher's manual $(1=$ considerably modified it, $2=$ slightly modified it, $3=$ followed it very closely). The scores per lesson were added up for each teacher and divided by the total number of lessons (6) to produce an average.

Continuation of current LLL was measured with 1 item: 'Do you intend on using the current Long Live Love program next school year for your sexual education lessons?' (1 $=$ no, certainly not, $5=$ yes, certainly). Teachers were asked in an open-ended question to explain their intention level.

Adoption of the revised LLL program was measured with 1 item:'Do you intend on using the revised Long Live Love program in the coming years for your sexual education lessons?' ( $1=$ no certainly not, $5=$ yes certainly). Adoption has been conceptualized as teacher's intentions to use the innovation in various other studies (Paulussen, 1994; Paulussen et al., 1994; Paulussen et al., 1995; Wiefferink et al., 2005). Teachers were asked in an open-ended question to explain their intention level.

\section{Independent variables}

Table 1 shows an overview of the independent variables, their internal consistency reliabilities, scales and items.

\section{Other variables}

In relation to Long Live Love, teachers were asked whether they used the DVD, teacher manual and student magazine in the LLL lessons $(1=$ yes, $0=$ no), how familiar they were with the program before using it and how many hours they had spent on teaching the LLL program. Extent of familiarity with the program was measured on a 4-point scale from (0) I only bought the program, to (3), I reviewed the program completely and thoroughly. 


\section{Statistical analysis}

Descriptive analyses were first conducted to get an overall picture of the research sample. Next, Pearson's correlation coefficient was used to reveal the correlations between the independent variables and the outcome variables (completeness, fidelity, intention to continue using current LLL and intention to adopt new LLL). Backwards stepwise multiple regression analysis was then used to identify factors associated with these outcome variables. All independent variables were entered at the same time for each outcome variable respectively. Only variables with significant bivariate associations $(p<.05)$ were included in the regression equations to understand how much variation in the outcome variables can be predicted by the independent variables. This regression analysis was done separately for each outcome variable. Several factors were dichotomized and included in the regression analysis as dummy variables, namely, school policy, descriptive norm and class composition. Multilevel regression analysis was not necessary because only one or two teachers per school participated in the study. Differences were interpreted as significant when $p<.05$. 


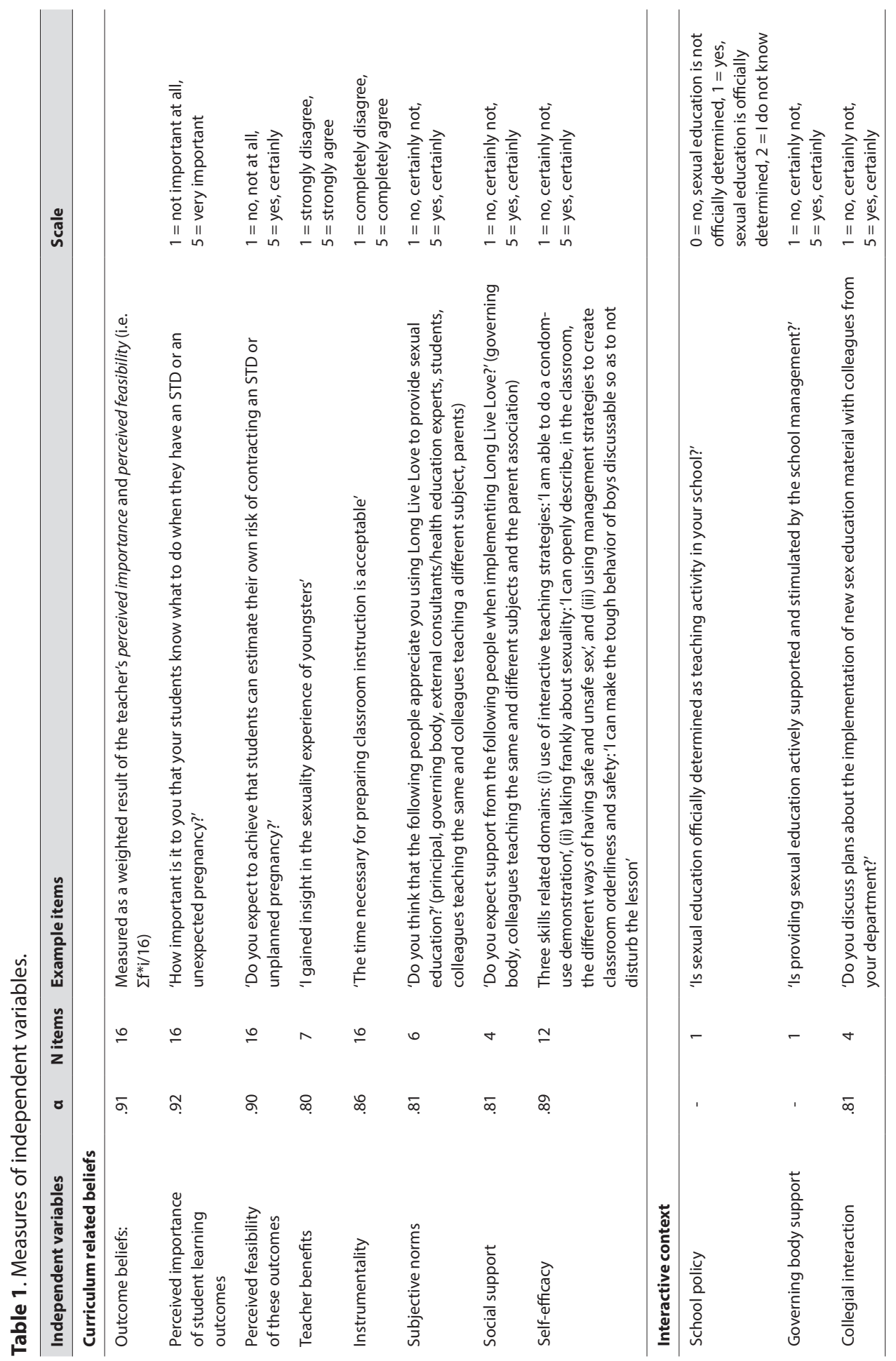




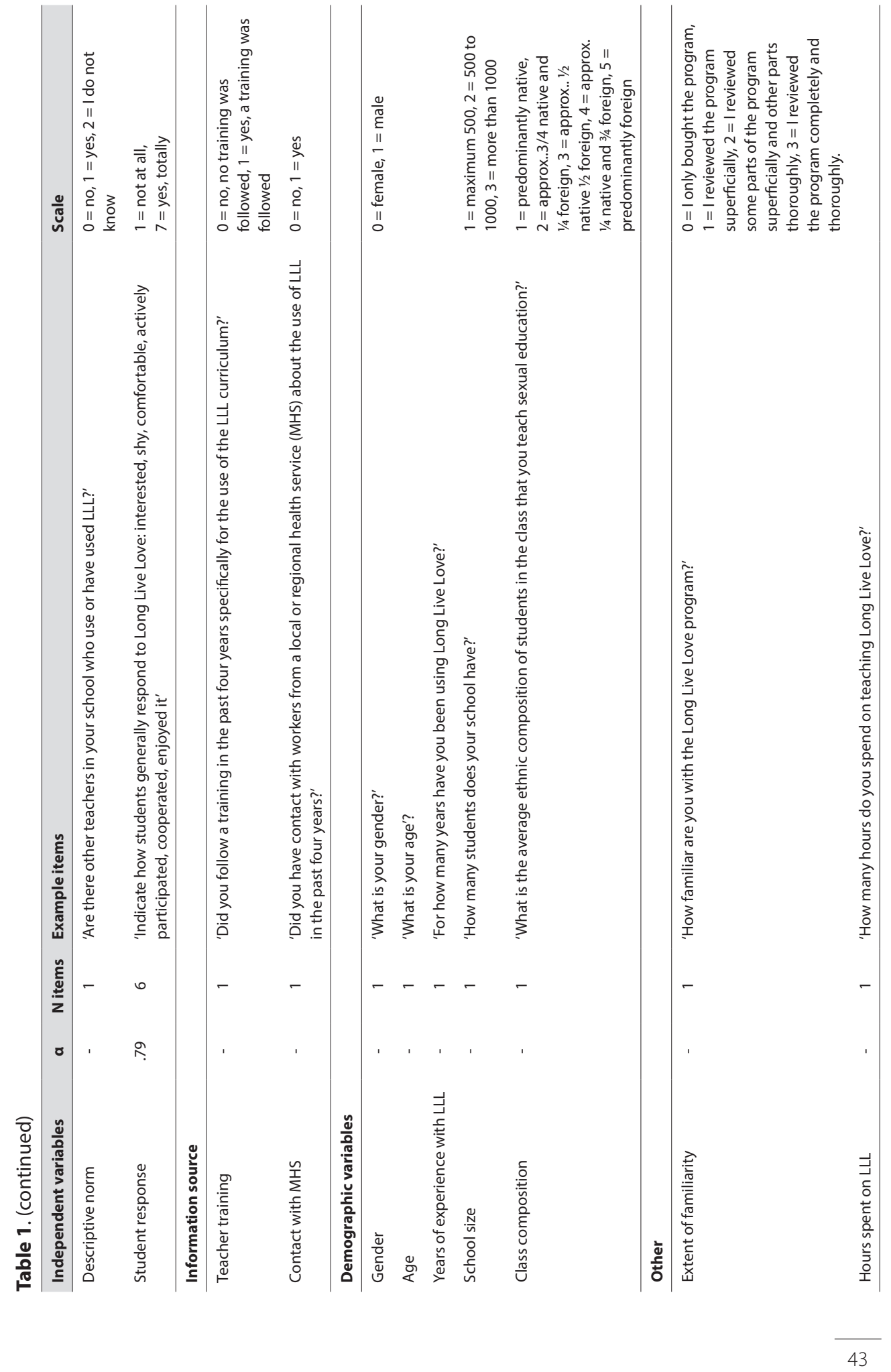




\section{RESULTS}

Means, standard deviations and inter-correlations of the study measures are shown in Table 2. The correlations and explained variances will be discussed per implementation stage to identify the most important determinants and gain insight into how much of the variance in the implementation stages can be explained by these determinants.

\section{Implementation of Long Live Love}

Approximately half the teachers report having relational and sexual education somehow formally established in school (55\%). More than half of participating teachers had not received a training in sex education at all (58.5\%) whereas $38 \%$ had received a training specifically for the use of LLL and 3.5\% had received a general training in sex education (not LLL specific). The majority of teachers (58.9\%) did not have any contact with the MHS in the past 4 years. Those who did have contact predominantly received a training specifically for LLL from the MHS (52.8\%).

Teachers generally spent 2 to 12 hours teaching the LLL program, depending on how much time they had available and needed to complete the program. Furthermore, teachers were familiar with LLL; the majority of them had reviewed the program completely and thoroughly before use $(59.7 \%, n=77)$. Few teachers only superficially reviewed the program $(6.2 \%, n=8)$ or solely bought it $(3.1 \%, n=4)$.

\section{Completeness}

On average, teachers implemented $64.1 \%$ (ranging 4.5-100\%) of the 22 learning activities included in the analyses. Each activity was completed by over $80 \%$ of the teachers except homework activities (ranging between 19-68\%). All components of the LLL program (student magazine, DVD, teacher manual) were used by over $90 \%$ of the teachers.

Completeness correlated significantly with numerous factors, namely teacher benefits, instrumentality, subjective norm, social support, and self-efficacy, student response, contact with the MHS, following a training specifically for LLL, spending more hours on LLL, fidelity and extent of familiarity with the program (see Table 2). Teachers were more likely to use more of the program if they saw benefits in its use for themselves, if they found the program practical to use, if they believed that others appreciate and support their use of LLL to give sexual education and if they believed they are capable of using LLL. They also used more of the program if they receive positive responses from students, are trained by the MHS in the use of LLL and if they were more familiar with 
the program. Additionally, teachers who spent more teaching hours on LLL and who delivered the program as prescribed use more of the program. The regression analysis revealed that $43.2 \%$ of the variance in completeness is explained by these determinants.

\section{Fidelity}

In general, teachers tend to follow the lessons as prescribed or slightly modify their lessons ( $M=2.1, S D=0.6)$. Especially lessons on risks of unsafe sex, negotiating condom use, and resisting social pressure to practice unsafe sex were considerably modified compared to the rest.

As shown in Table 2, the most important correlates of fidelity are instrumentality, selfefficacy, training for LLL, years of experience and extent of familiarity with LLL. Teachers are more likely to implement the program as prescribed if they find the program to be practical and useful in practice, if they believe they are capable of using LLL, if they follow a training specifically for LLL and if they were more familiar with the program. On the other hand, teachers who have worked with LLL for longer years appear to modify their execution of the program and diverge from the prescription. The regression analysis indicated that $25 \%$ of the variance in fidelity is explained by these determinants. 


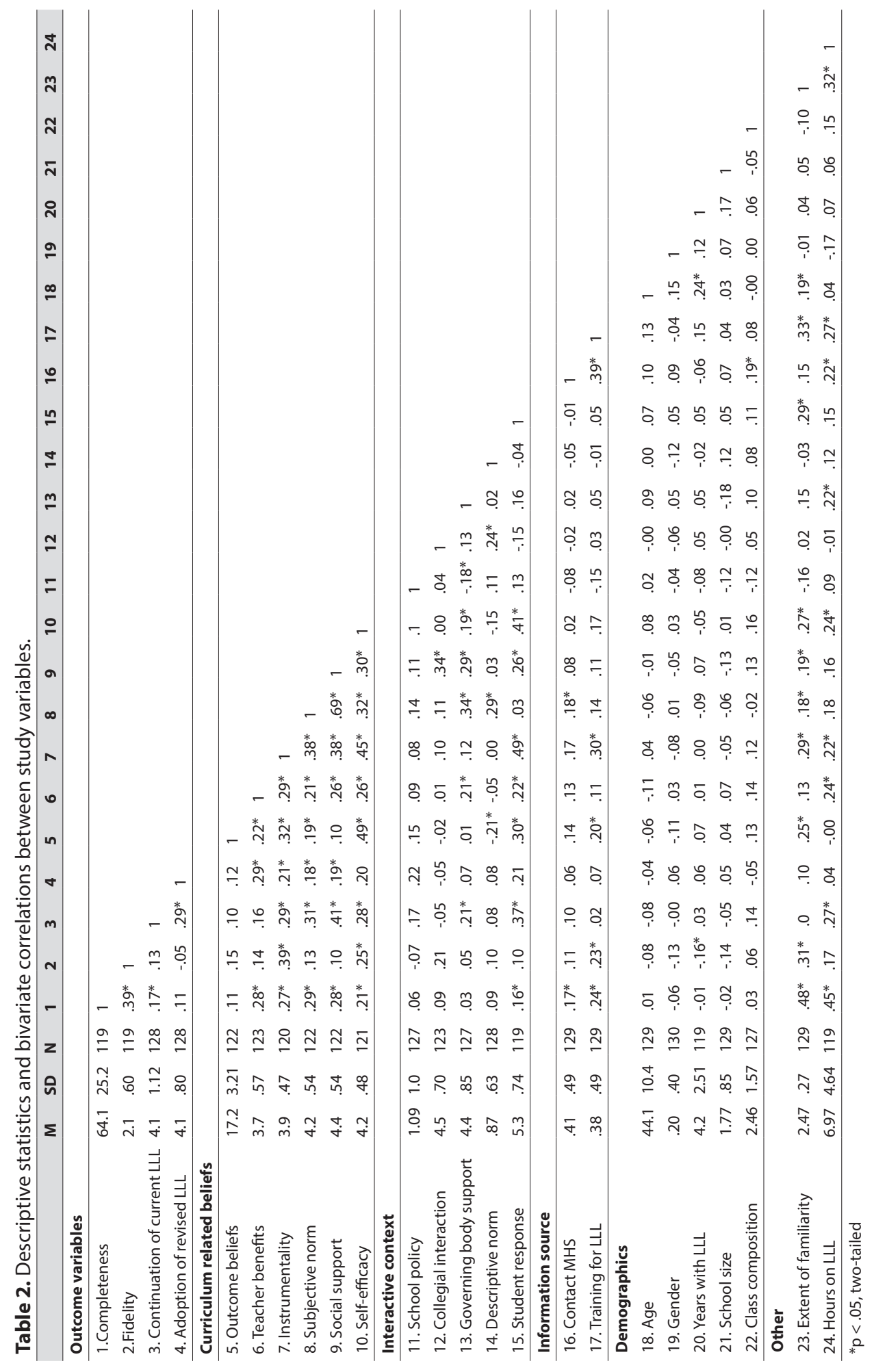




\section{Continuation of current Long Live Love}

The intention level of the group to continue using the current LLL was generally high (mean $=4.1 ; S D=1.12$ ). Factors that appear to significantly predict and explain intention to continue using LLL are instrumentality, subjective norm, social support, self-efficacy, student response, governing body support, school policy, completeness and hours spent on LLL, as shown in Table 2. These variables predicted $30.2 \%$ of the total variance in intention to continue using the current LLL. Consequently, teachers are more likely to re-use the current LLL program if they find the program practical, believed that others appreciate and support their use of LLL to give sexual education, and if they believe they are capable of using LLL. Receiving positive responses from their students, experiencing support from the school management in their implementation of $L L L$, and established school policy for sex education, and taking more time for teaching LLL also predict higher intentions for continuation of LLL.

Explanations for intention level were reported by teachers in the open-ended questions of the questionnaire $(\mathrm{N}=91)$. Teachers with higher intentions to continue using the current LLL were more positive about the curriculum (34\%) and believed it appealed to students (20.9\%). Other teachers are happy to use this program until something better appears on the market (12.1\%). Explanations for lower intention levels are that teachers find the material outdated, especially the DVD, and prefer to wait for a new version (13.2\%). Some teachers claimed the material no longer appeals to students due to being outdated $(6.6 \%)$, while others found the program lacking modern-day information (5.5\%) or found the program too time consuming (4.4\%). Some teachers simply were no longer teaching subjects in which LLL was usually provided (3.3\%).

\section{Adoption of 'revised' Long Live Love}

The intention to use the revised LLL was high (mean $=4.1 ; S D=0.80$ ). Table 2 shows the predictors of intention to use the new LLL program, namely teacher benefits, instrumentality, subjective norm, and social support, followed by intentions to continue using the current LLL version. These factors explained $23 \%$ of the variance in intentions to adopt the new LLL.

Explanations for a level of intention to adopt the revised LLL program were reported by teachers in the open-ended questions of the questionnaire $(N=74)$. Teachers with higher intentions to adopt the revised LLL program namely had hopes and expectations that the new program will be an improvement on the previous version (modern, appealing 
to students and enriched with current issues) (52.7\%). Lower intention levels to adopt the new LLL can be explained by teachers' uncertainty about the content of the new program and a preference to judge for themselves first (47.3\%).

\section{DISCUSSION}

The current study has attempted to provide insight into the promoting and inhibiting factors of adoption, implementation, and continuation of the school-based sex education program, Long Live Love. Different factors influence each stage in the implementation process and understanding factors influencing each of these stages is essential for successful implementation (Sy \& Glanz, 2008). The different stages are, however, dependent on one another and complementary.

A positive result of this study is that most of the LLL program is delivered and that teachers generally do this with relative integrity. On average, teachers carried out approximately two-thirds of the activities to be implemented from the program and delivered the lessons as prescribed or only modified them slightly. This is a promising result as several studies indicate that programs are frequently modified during implementation (Berman \& McLaughlin, 1976; Ringwalt et al., 2003; Rogers, 2003) and teachers do not always implement programs according to specific guidelines (Sy \& Glanz, 2008). Intentions to continue using the current LLL were relatively high as was the intention to adopt the revised LLL.

Teachers curriculum related beliefs were found to be important for all stages of the implementation process. Implementation (completeness and fidelity) was especially related to following a training specifically for $L L L$, greater instrumentality of the program, higher self-efficacy and greater familiarity with the program. Teachers who followed the guidelines of the LLL program more closely (fidelity) also completed more of the program (completeness). These findings are similar to a process evaluation study of a schoolbased adolescent sexual health intervention in rural Tanzania, where teachers delivered the program to primary school students with remarkable integrity and this fidelity was enhanced by a training course (Plummer et al., 2007). Teacher curriculum related beliefs and information source variables are therefore essential for implementation (Borko, Livingstone, \& Shavelson, 1990; Schaalma, Kok, \& Poelman, 1994).

Fidelity was, however, hindered in our study when teachers had more years of experience with LLL. Several studies indicate that programs are frequently modified in the process of implementation (Berman \& McLaughlin, 1976; Ringwalt et al., 2003; Rogers, 2003; 
Sy \& Glanz, 2008). Years of experience with a program may lead to reinvention of it by the user to accommodate the changing circumstances in schools and diversity in composition of classrooms (gender, ethnicity or sexual experience of students) in time (Berman \& McLaughlin, 1976; Ringwalt et al., 2003; Rogers, 2003).

Continued use of LLL was positively related to interactive context variables (student response, governing body support and having a school policy for sex education formally established), and curriculum related beliefs (instrumentality, subjective norms, social support, and self-efficacy). Furthermore, the more of the curriculum the teacher completed and the more hours they spent on LLL, the higher the intention to continue using LLL. Information source variables did not correlate with continued use.

Adoption of the revised LLL was predominantly related to curriculum related beliefs, namely teacher benefits, instrumentality, subjective norm, and social support. Also, teachers who had a higher intention to continue using LLL were also more likely to adopt the new LLL. Information source variables and interactive context variables did not correlate with adoption. Self-efficacy correlated with adoption but not significantly. This runs counter to the findings of other studies, in which self-efficacy appeared to be a dominant predictor of teachers' decision making on innovations (Wiefferink et al., 2005). Perhaps with a higher power, self-efficacy would have been found to be significant. One possible explanation why self-efficacy was not a dominant predictor of adoption might be based on the correlations between outcome beliefs, instrumentality subjective norms, and teacher benefits on the one hand, and self-efficacy on the other hand. This would correspond with the theory of Bandura that self-efficacy predicts outcome beliefs and other cognitions and that these factors in turn predict behavior (Bandura, 1986). Another explanation might be that teachers' efficacy is less dominant during the stage of adoption than during implementation (Sy \& Glanz, 2008).

To date, only one study is comparable to the current one: the SHARE program in Scotland (Buston et al., 2002). The SHARE (Sexual Health and Relationships: Safe, Happy and Responsible) study examined factors that impeded or facilitated the implementation of a teacher-delivered sex education program for youth (13-15 years old). Results showed that fidelity was aided by intensive teacher training, classroom compatibility, and senior management support while it was hindered by competition for curriculum time, brevity of lessons and teachers' limited experience and ability in use of role-play (Buston et al., 2002) Paulussen (1994) found that teacher's adoption and implementation behavior of HIV programs were most strongly related to teacher beliefs (attitudes, social influences, self-efficacy), as this study also suggests. Generally, teachers will teach best in areas for 
which they are best prepared, have effective materials and techniques, and for which they receive recognition and support from school administrators and colleagues (McCormick et al., 1995).

Results of this study show that adoption is predominantly related to individual level factors, whereas implementation and continuation are also influenced by external factors, namely information source variables and the interactive context, respectively. Teacher training is an information source variable that is especially important in stimulating complete and correct use of LLL. It has been identified previously as a major determinant of success in the implementation of school-based programs (Connell, Turner, \& Mason, 1985; Nelson, Poehler, \& Johnson, 1988). Pre-implementation training has been found to increase the integrity with which teachers implement a curriculum (Flay et al., 1987; Perry, Murray, \& Griffin, 1990; Ringwalt et al., 2003; Ross, Luepker, \& Nelson, 1991) because it enhances teachers' skills that are relevant to the intervention program (Han \& Weiss, 2005). In the Netherlands teacher training in sexual health promotion is provided by the MHS.

Continued use of LLL is largely dependent on conditions that enable structural embedding of LLL, namely a supportive school management and school policy formally establishing sexual education in the school. Also, observing positive student responses reinforces implementation behavior of teachers (Paulussen et al., 1995). In South Africa, ongoing engagement and support of teachers were also found to play an important role in their ownership of an AIDS prevention curriculum and partially explained continued use of the program (Ahmed et al., 2006). Support motivates teachers to implement the program, and in doing so correctly, they are likely to experience further success in changing their students' behavior in the classroom, which in turn leads to continued program use (Han \& Weiss, 2005). School policy has also been found to be essential in contributing to a successful implementation process (Campbell \& Lubben, 2003; Paulussen et al., 1995; Schaalma et al., 1996).

The study had some limitations. Due to the cross-sectional design of this study, conducted at one moment in time, it is impossible to determine whether the teacher curriculum-related beliefs precede teacher's implementation behavior or if they are a result of it. No present conclusions can be drawn about causality, only associations, unless a longitudinal study is conducted. Also, the same measures were used to predict implementation and continuation of the current LLL as adoption of the 'revised' LLL, which could limit interpretations of the adoption results. Past experiences with a program have, however, been found to predict future use (Paulussen et al., 1995; Han \& Weiss, 2005; Johnson et al., 2003; Levenson-Gingiss \& Hamilton, 1989). 
Additional methodological limitations of the study are self-reports by teachers, a self-selected sample, limited measurements on the outcome measures of adoption and continuation with a single item and lack of assessment of student outcomes. An effectiveness study was, however, conducted in 2001, where LLL was found to have positive outcomes for students, proving that it is a worthy program (Schaalma et al., 2002). Process evaluation remains essential for examining the quality and extent of program implementation and understanding the effects of interventions (Wight \& Obasi, 2003). Observation of fidelity and rapport would have further validated the results of this study.

With this study, we hope to share lessons for successful implementation in the school setting. The results reveal that each stage of the implementation process is influenced by different kinds of factors. This implies that it is necessary to consider all three stages when planning and evaluating the implementation of interventions. It also implies that different strategies are needed to enhance adoption, implementation, and continuation of an innovation as the Diffusion Theory suggests (Rogers, 1995). To enhance adoption, the focus should be predominantly on teacher curriculum-related beliefs, presenting the personal benefits of using the intervention, providing support for use of it and developing a practical and easy-to-use intervention. Implementation is further supported by equipping teachers with knowledge and skills through training to promote quantity and quality of implementation. Continuation is attained by a supportive school policy and climate of personal support for teachers (Schaalma et al., 1996). The implication for health education is that in addition to addressing more traditional factors such as training, and teacher beliefs, the program planners should also consider the climate of the organization (Parcel et al., 2003). These broader contextual factors may support or inhibit teacher's efforts at program implementation (Han \& Weiss, 2005).

Much needed insight has been provided for the facilitating and inhibiting factors influencing the different stages of the implementation process of a school based sex education program, Long Live Love. This information becomes especially relevant in the field of health promotion intervention development, where the importance of implementation is being increasingly acknowledged (Bartholemew et al., 2011). Understanding the determinants of the implementation process of LLL will not only benefit the extent and quality of implementation of the future updated Long Live Love program or provide inspiration for the systematic development of an implementation strategy but also provides possible explanations for effectiveness of such curricula and why these may succeed or fail when conducted in a real-world setting. The suggested 
recommendations may lead to improved implementation of school-based sex education programs internationally and locally, contributing significantly to a better-equipped and knowledgeable youth concerning sexuality and relations. Long live love! 



\title{
CHAPTER 3
}

\section{Support from the Municipal Health}

\section{Services (MHS) in implementing}

school-based sex education:

\author{
enabling and inhibiting factors
}

\section{in times of economic cutbacks}

This chapter is published as:

Schutte, L., Mevissen, F.E.F., Kok, G. (2014). Ondersteuning vanuit de GGD bij implementatie van seksuele voorlichting op school: faciliterende factoren en barrières in een tijd van bezuinigingen / Support from the Municipal Health Services (MHS) in implementing school-based sex education: enabling and inhibiting factors in times of economic cutbacks. 


\section{ABSTRACT}

Schools are the ideal setting to reach many youngsters at once with health promotion programs. The implementation of such programs is, however, not an automatic process and requires external assistance. Even though there is often support from the Municipal Health Services (MHS), their role is no longer obvious due to the current economic crisis. Surveys and interviews were used to research what the current and future supporting role of the MHS is and could be in the implementation of Long Live Love (LLL); a schoolbased sex education program for secondary school students. Results reveal that the MHS does increasingly less in supporting the implementation process of the previous LLL program. They are, however, prepared to continue supporting the dissemination and adoption of the new LLL program. Promoting and inhibiting factors influencing their supportive role in the implementation process of LLL are categorized into (1) characteristics of the (previous) LLL program and (2) influence of i) teachers, ii) schools, iii) the MHS as an organization and iv) the municipalities. The outcomes of this research can contribute to the development of an implementation strategy for health promotion interventions in schools, even when the MHS or other school-supporting organizations can only offer limited support. 


\section{Key Issues}

- Implementation of health promotion programs in schools is not an automatic process. External support is needed and this often comes from the MHS.

- Barriers and enabling factors for the implementation and continuation of implementation promoting activities by the MHS are related to characteristics of the curriculum and the role of teachers, schools, MHS, and municipalities.

- The current budget cuts limit the supporting role of the MHS to promoting the dissemination and adoption of the new Long Live Love curriculum.

- The MHS remains an important and appropriate actor in the implementation of school health promotion programs.

- In addition to a revised LLL curriculum, an implementation-promoting strategy is needed, aimed at factors that influence the supporting role of the MHS. 



\section{INTRODUCTION}

Schools are a convenient place to reach many young people simultaneously with health promotion programs. The effects of these programs on the health of youth are, however, dependent on the quality of the program itself, as well as on the quality of the implementation: from dissemination (being aware) to adoption (intention to use), implementation (actual use), and ultimately continuation (continued use). Implementation is, however, not an automatic process. Supporting schools and teachers is necessary for optimal implementation and strengthens effectiveness (Bos, de Jongh, \& Paulussen, 2010). In the Netherlands, the Municipal Health Service (MHS) has the responsibility for supporting health promoting activities in schools and plays an important role in the dissemination of health promotion programs, and supporting adoption and (correct) implementation of these by teachers (Bos et al., 2010). However, the MHS also needs to take an adoption- and implementation decision for themselves; they also need to decide which programs and related implementation promotion activities they need to take on and implement. We examined the current supporting role of the MHS and their potential future role and what factors influence this role, with regards to a sexual health-promoting school program, 'Lang Leve de Liefde' or Long Live Love (LLL). The previous support from the MHS has ensured proper implementation of LLL during the SLIM (Systematic National Implementation) project in 2002 (Fulpen et al., 2002; Wiefferink et al., 2002). Given the current economic crisis, this role may have changed, which would require alternative forms of support for schools / teachers in the implementation of health promotion programs.

\section{The LLL curriculum}

LLL is the most successful intervention on relationships and sexuality for secondary education (Vanwesenbeeck et al., 2003). It has existed for more than twenty years and has recently been revised for the fourth time. Use of the previous curriculum gradually declined, the main reason being that the program material was outdated (Schutte et al., 2014). The research described in this paper was conducted in support of the planned development of an intervention strategy to promote the adoption and implementation of the new fourth version of LLL.

\section{The role of the Municipal Health Service}

The Diffusion of Innovations Theory describes implementation as a process; from dissemination and adoption to implementation, and continuation (Rogers, 1995). Several factors affect each stage of the process, namely (1) characteristics of the adopters and users of the innovation (2) some environmental factors (Rogers, 1995). 
In the case of LLL, teachers have to adopt the curriculum and implement it (continuously). The MHS conducts adoption, implementation, and continuation promoting activities to support teachers in this process and thus to optimize the implementation of LLL. To perform these activities, the MHS must first decide whether they want to take on these implementation-promoting activities to begin with (adoption of implementation promoting activities). The MHS should then properly implement these promotional activities, namely stimulate teachers to adopt, implement, and continue the health promotion program and continuously implement these implementation-promoting activities, also for the new LLL.

When the MHS does not adopt a specific school program and the promotional activities around it and subsequently does not implement or continue these activities, this can be detrimental to the quality of the implementation of the school program by teachers. In this study, we have therefore attempted to identify what factors influence the willingness and ability to (continue to) implement these activities. The research focuses on the role of the MHS, as the MHS professionals perceive it, and their perceptions of enabling and inhibiting factors.

\section{METHOD}

Quantitative (surveys) and qualitative (interviews) research methods were simultaneously conducted in a cross-sectional study. The survey focused on what activities the MHS conducts around promoting the implementation of the previous LLL, the possible future role in the new LLL and barriers and enabling factors in this. The interviews were complementary to the questionnaire, with special attention for enabling and inhibiting factors.

\section{Population and recruitment}

A total of 83 MHS professionals were approached (all involved with LLL in the past) across all 28 Municipal Health Services in the Netherlands. Eventually 26 MHS professionals from 25 MHS-es participated in the survey, of which nineteen also participated in the interviews (14 females, 5 males). Drop-out was caused by illness or no longer working at the MHS, no longer involved with LLL, or already being represented by a colleague in the study. Respondents were mainly public health officials and STI social nurses. The number of years worked at the MHS ranged from 1 to 26 years $(M=7.9 ; S D=5.2)$. Level of experience around the implementation of LLL varied between the participating MHS professionals. 


\section{Procedure}

Participants received an email with a link to an online survey (completion time \pm 10 minutes) with questions about adoption, implementation and continuation of the 'LLL implementation promoting activities'. The data was analyzed with SPSS 17. Appointments were scheduled with MHS professionals who were willing to be interviewed at their own MHS location (interview duration 1 hour). Interviews were analyzed using MAXQDA.

\section{Measuring instruments}

The online survey and the interview protocol are based on the Diffusion of Innovations Theory (Rogers, 1995). For an overview of measures and concepts in the survey and interview, see Table 1.

\section{RESULTS}

The results of both the survey and the interviews are reported and resulted together below in Figure 1. The results reported are based on judgments made on a personal basis by the interviewed MHS professionals and do not represent the views of the MHS as an organization.

\section{Embedding sexual health promotion within the Municipal Health Service}

The subject of 'sexual health' is explicitly mentioned as a priority in the municipal public health policy in twelve of the participating municipal health services, eight do not have it as a priority and for five of the MHS-es it is unknown. Four MHS-es no longer support schools in sexual health, 21 do, of which nineteen promote LLL.

\section{Current adoption and implementation of LLL}

In the last three years, LLL was promoted by most Municipal Health Services $(N=19)$ with an average of fifteen schools per MHS department (range: 0 to 80; total number of schools: 289; mode: 5 schools). Each MHS spent an average of 75 hours a year in assisting schools in LLL (range: 0 to 530 hours; mode: 100 hours). Most MHS-es informed $(\mathrm{N}=21)$ and stimulated $(\mathrm{N}=18)$ schools to use the curriculum. Nearly half $(\mathrm{N}=13)$ trained teachers in the use of LLL and two MHS-es observed teachers or organized consultation meetings for teachers. Eight MHS-es conducted other LLL-related activities (e.g. parent-teacher meetings, STI guest lectures, providing free educational materials and evaluation exercises). 
Table 1. Concept from survey and interview.

\begin{tabular}{lll}
\hline Concepts & Questions & \multicolumn{1}{c}{$\begin{array}{c}\text { Survey or } \\
\text { interview }\end{array}$} \\
\hline $\begin{array}{l}\text { Demographic } \\
\text { information }\end{array}$ & $\begin{array}{l}\text { Name and region MHS, gender, age, work experience (department, function, } \\
\text { years of working experience) of participants }\end{array}$ \\
\hline $\begin{array}{l}\text { Embedding sexual } \\
\text { health promotion } \\
\text { within the MHS }\end{array}$ & $\begin{array}{l}\text { If the theme 'sexual health' is included as a priority in the municipal public Survey } \\
\text { health policy, if the MHS supports schools in sexual health promotion (no, } \\
\text { yes, do not know), and which teaching materials are promoted to schools in } \\
\text { this field of sexual health (no teaching material, Long Live Love, other teaching } \\
\text { material) }\end{array}$ \\
\hline
\end{tabular}

\section{LLL implementation- promoting activities by the MHS}

Adoption of LLL Number of schools to which LLL was promoted in the past 3 years
'implementation-
promoting activities'

Implementation of Number of hours spent on supporting schools in the introduction of LLL Survey

LLL'implementationpromoting activities'

Which supporting activities (now or in the previous years) are offered to Survey schools for the introduction of LLL (Inform schools about the existence of LLL; stimulate schools to purchase/use LLL; Train teachers in the use of LLL; Observe teachers and provide them with feedback on their use of LLL; organize consultation meetings for teachers; Other)

Continuation of the new If the MHS wants to play a future role in the introduction of the new LLL'implementationLLL curriculum in schools in their region (yes, no, do not know) and promoting activities' which supporting activities they could execute for the promotion and implementation of the new LLL curriculum in secondary schools: (see options in table 3 ) and hours available to spend next year on supporting schools with the introduction of the new LLL curriculum.

\section{Barriers and enabling} factors
Knowledge and skills to train teachers in preparation for the use of the new LLL curriculum, need to participate in a train-the-trainer workshop for MHS in preparation of training and supporting teachers who will use the new LLL curriculum, attitudes towards participating or not participating in the promotion of implementation process of LLL among teachers in schools (6 statements: strongly disagree-strongly agree; characteristics of the curriculum, the role of teachers, schools, MHS and municipalities) and desires as to what needs to change in order to optimize the implementation of the new LLL curriculum (nothing, the situation is fine as it is; More collaboration between the departments within my MHS organization; make the theme sexual health more of a priority within my MHS organization; My department needs more support from STI Aids Netherlands in the delivery of our activities to schools; Other)
Survey \& interview 


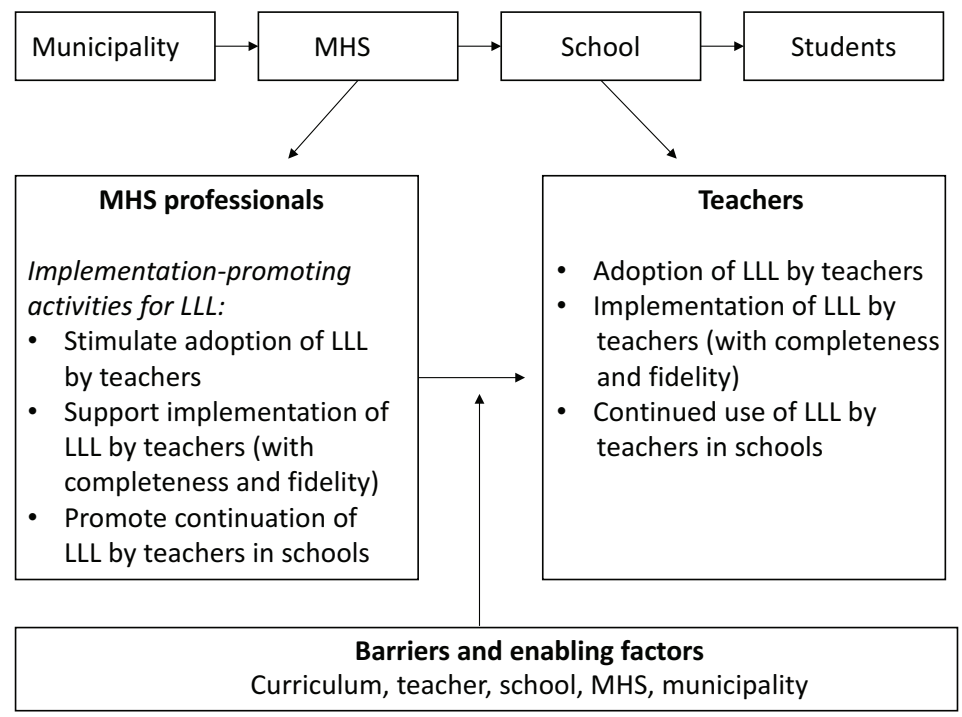

Figure 1. Supporting role of the MHS in the implementation of LLL.

A group of five MHS-es did nothing in the past three years around promoting adoption or implementation of LLL by schools. Another group of five MHS-es promoted adoption but not implementation of LLL.

\section{Enabling and inhibiting factors}

Factors that promoted or prevented the adoption-, implementation- and continuation promoting activities of the MHS around LLL are related to two categories: (1) characteristics of the curriculum, (2) the role of teachers, schools, MHS-es and municipalities. See Table 2.

\section{Characteristics of the curriculum}

Barriers. Many MHS-es are waiting for the renewed LLL, meaning that that they no longer promote the previous LLL in schools.

MHS 1: "LLL is simply outdated. ... and that is basically the reason for me not to promote the use LLL as a curriculum."

Teachers found the current curriculum too multicultural, the scope too large and the time investment too much. These characteristics of the curriculum negatively influenced the decision of teachers to use it, making it difficult for the MHS to promote the curriculum to teachers. 
Enabling. LLL was a complete program that was scientifically based, which was important to the MHS and teachers.

MHS 1: "Because it's actually the only good teaching material there was, that existed...there were all these separate things and now there was this one big, good, complete curriculum. As MHS you naturally choose curricula that are good and that are scientifically based and that is the advantage of this curriculum."

\section{Teachers}

Barriers. According to the MHS many teachers found sexuality a difficult subject to discuss, they were embarrassed and had norms and values that were not consistent with those of LLL or did not find the subject important enough, making them less inclined to accept LLL from the MHS.

MHS 3: "I noticed that teachers felt resistance in talking to young people about sexuality."

Teachers who found it challenging to cope with difficult situations while teaching about sexuality (e.g. dealing with personal questions from students), also received limited support from the MHS due to lack of time, and didactic expertise. The consequence being that teachers did not fully or correctly implement LLL.

Enabling. Adoption was facilitated when the MHS investigated, together with the teachers, what the obstacles were to use LLL and what other possibilities there might be around sex education. The MHS teacher training in which dealing with difficult situations in the classroom was practiced, seemed to promote the implementation of LLL.

\section{Schools}

Barriers. According to the MHS there was a lack of structural integration of sexual and relational education in general and of LLL in particular, in education. This meant that the decision to (continue) use or not to use LLL often depended on one teacher, which was problematic with staff turnover. There was no time allotted for LLL in the annual school plan; teachers forgot to schedule LLL and had little time. Sexual education was highly dependent on biology teachers and mentors. Lack of time and work overload often forced teachers to work on 'theme days' and 'project weeks', in which LLL did not always fit.

MHS 4: "It drove teachers crazy, all these fragmented activities. So then it's; LLL has to fit into the project week. If it does not fit in there, then nothing happens that year." 
MHS professionals had fewer requests from schools because many teachers were already familiar with LLL.

MHS 2: "The fact that it is already known to other schools is a very important argument because you do not have anything new to report. Then it is more purely informative; how do you like it, can you still use it, are you still interested ..."

Some schools used a different curriculum altogether which they found satisfactory. Furthermore, sexuality was sometimes difficult to discuss, especially in religious regions.

Enabling. The adoption-promoting role of the MHS was stimulated by the use of existing entrances in schools (e.g. via school nurses). Structural embedding of sex education in the curriculum and its overlap with the school's attainment objectives (note a) made it easier for teachers to deal with sexuality and to create support within the school.

\section{MHS}

Barriers. The biggest problem for MHS professionals was lack of time within the department.

MHS 5: "Look, I think the biggest problem is the lack of time at this department."

MHS-es where sexual health was not a priority also did less to support the implementation process. In addition, there was a lack of collaboration between MHS departments and therefore, insufficient information and lack of clarity about who was responsible for sex education and LLL; sometimes it depended on one person.

MHS 1: "I think that the theme fits with the MHS, it has the MHS' attention and that it varies per MHS but that it fits somewhere, in one of the departments. But the question is whether it is a major interest and whether it really is a priority for the MHS."

MHS-es did not want to offer outdated programs to overburdened schools, they had difficulty to trace the correct contact person in the school and they approached schools possibly at the wrong time.

MHS 6: "It can sometimes be tricky before you get the right person."

MHS professionals also had little insight in what happened to the curriculum after being purchased because they had no monitoring system. 
Enabling. Strong collaboration between the various MHS departments was conducive to stimulating the adoption and creating support for LLL amongst teachers. National support of the MHS in the implementation of LLL (SLIM) worked favorably. Also, a demand-oriented 'Health Promoting School' (note b) approach of MHS (Hoekstra et al., 2009) made the step for recruiting schools easier. Furthermore, follow up calls with schools allowed the MHS to monitor what was happening with the curriculum in their region. Preservation of LLL promotion was easier whenever the MHS had something new to offer in the field of LLL to schools.

\section{Municipality}

Barriers. MHS professionals informed us that sexual health was not a priority in many municipalities and was not included in the municipal public health policy (note c). Financial cuts meant that some municipalities had no funding for sex education in schools. These factors limited the extent to which the MHS could support the implementation process of LLL.

MHS 6: "How much we support the implementation of LLL depends on the person, as with all programs; some people are more convincing than others. And it also depends on the available financial resources, because you are obviously dependent on that. And thirdly depends on which problems are prevalent at the time."

Enabling. MHS could promote the continuation of LLL in schools when they urged municipalities to prioritize sexual health in the region. Municipalities then provided the money. 


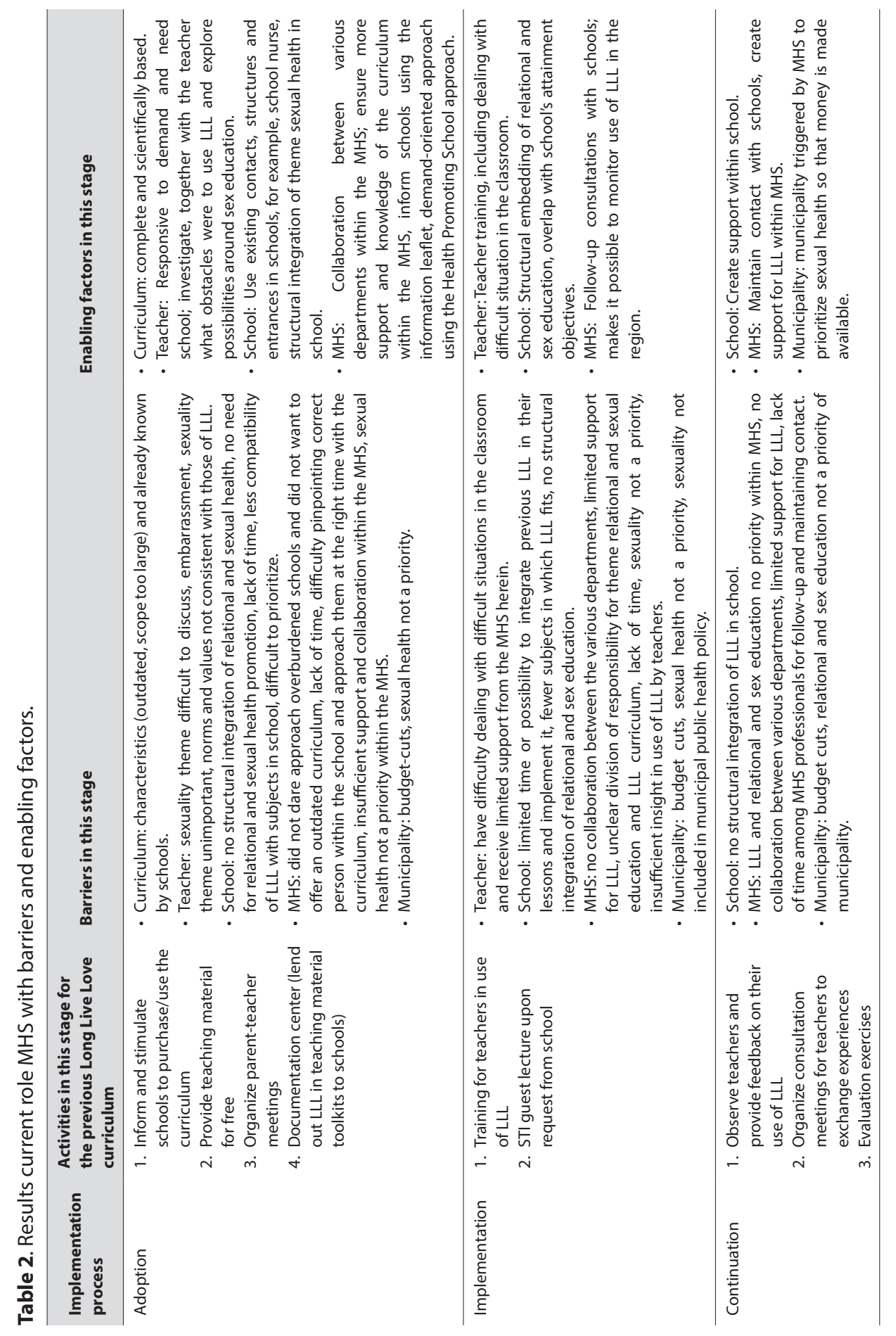




\section{Future adoption and implementation of LLL}

A summary of the results with regard to the future supporting role of the MHS is presented in Table 3. Most MHS professionals $(\mathrm{N}=22)$ indicated that they want to play a role in the introduction of the new LLL curriculum because sexual development is an important topic within their department. The exact contours and content of this role appeared to depend on the financial situation within the MHS. Four MHS professionals wanted to first know how the new curriculum would look like.

MHS professionals want to focus primarily on promoting dissemination and adoption. To encourage this, they wanted to bring the curriculum to the attention of teachers through personal contact $(\mathrm{N}=14)$. Also through the use of resources such as information leaflets $(N=19)$, regional information workshops $(N=12)$, and lending out teaching material toolkits (note $d)(N=3)$. They also wanted to involve parents $(N=10)$. Some MHS professionals $(\mathrm{N}=4)$ wanted to work according to the 'Health Promoting School' approach (note c) and provide support that meets the needs of the school. MHS-es with more financial resources wanted to offer a teacher training $(N=13)$. More than half of the MHS professionals $(N=15)$ believed that LLL teacher training was part of their job.

For the implementation and continuation of LLL in schools, MHS professionals indicated they could not do much. They did not want to invest in intensive LLL-related relationships with schools, except in the form of giving short workshops about LLL and sending program updates.

On average, there would be 52 hours per year per MHS available for implementation promoting activities around the new LLL curriculum (range 0-530 hours; mode: 50 hours), which is less time than they had before; 2 MHS-es stated they could not spend time on it next year.

\section{Barriers and Enabling factors}

Involvement and the future role of the MHS in the continued support of the implementation process of the new LLL in schools is dependent on the curriculum, and the role of the school, the MHS, and the municipality. 
Table 3: Mentioned implementation-promoting activities for the revised LLL.

\begin{tabular}{|c|c|c|}
\hline Implementation process & Implementation-promoting activity & $\begin{array}{l}\text { Number of MHS } \\
\text { professionals (\%) }\end{array}$ \\
\hline & No supporting activities & $0(0)$ \\
\hline \multirow[t]{5}{*}{ Dissemination \& Adoption } & $\begin{array}{l}\text { Using materials to introduce the curriculum to schools and teachers } \\
\text { (for example, overview of materials, information leaflet, posts on } \\
\text { websites) }\end{array}$ & $19(73)$ \\
\hline & $\begin{array}{l}\text { Introduce the curriculum to schools and teachers by means of } \\
\text { personal contact }\end{array}$ & $14(54)$ \\
\hline & Organize regional information workshops for schools and teachers & $12(46)$ \\
\hline & Organize a parent-teacher meeting for parents of students & $10(38)$ \\
\hline & Other activities & $7(27)$ \\
\hline \multirow[t]{2}{*}{ Implementation } & $\begin{array}{l}\text { Train teachers in the use of the curriculum, for example, } 2 \text { days per } \\
\text { year }\end{array}$ & $13(50)$ \\
\hline & $\begin{array}{l}\text { Observe teachers and provide feedback (to improve their lesson) } \\
\text { during the use of the curriculum }\end{array}$ & $4(15)$ \\
\hline \multirow[t]{2}{*}{ Continuation } & $\begin{array}{l}\text { Periodically inform teachers about the latest updates around the } \\
\text { curriculum using newsletters }\end{array}$ & $16(62)$ \\
\hline & $\begin{array}{l}\text { Organize consultation meetings } 1 \text { to } 2 \text { times a year for teachers } \\
\text { using LLL }\end{array}$ & $7(27)$ \\
\hline
\end{tabular}

Barriers: Schools need money, time and to have interest in LLL and sexual health to be included in the core educational objectives (note a). In addition, several MHS professionals $(\mathrm{N}=12)$ stated their uncertainty of having sufficient knowledge and skills to provide training for teachers in the use of the new curriculum. They wanted to first follow a training themselves. Due to time constraints and the need for a MHSuniform LLL-approach, they wanted to get material and didactic support from national organizations, such as from STI Aids Netherlands in the form of ready-made materials to assist them in implementation promoting activities of the new LLL, such as a (standard) PowerPoint presentation for teacher training, recruitment letters and a LLL information leaflet. MHS-es also wanted insight into what happened with LLL in schools after purchase.

MHS employees $(\mathrm{N}=10)$ further expressed that more support was needed within the MHS for the theme of sexuality plus better cooperation between the various departments, as well as the possibility of sharing experiences with other municipal health services. Some $(\mathrm{N}=7) \mathrm{MHS}$ professionals emphasized that sexuality had to be a priority, with enough time and money, and structurally embedded in the plan of action of the MHS. 
Finally, the municipal public health policy does not prioritize the introduction of such teaching materials in schools. According to the MHS, sexual health should be in all municipal public health policies so that money becomes available and LLL can be widely deployed.

Enabling: The most important reason to continue supporting the implementation of LLL was that they liked the current curriculum and expected the future curriculum to also be good, timely, effective, and scientifically based.

MHS 7: "And I think it is important for us that we see the content of the curriculum is, what the added value is and its uses. That's important, I think for one who promotes it. But we do know that it is an evidence-based program. That is extra motivation to pursue it."

Furthermore, MHS professionals expect to be able to perform the main tasks expected of them from the municipality, in the area of sexual health by using the new LLL. Eighteen MHS professionals found that the new curriculum needed to support them content wise in achieving their goals around sexual health.

MHS professionals hoped to be able to profile themselves as a supportive institute for schools. It also gave them personal satisfaction to work with schools to introduce LLL. Improving their knowledge and skills in the field of educational support was found to be less important.

\section{DISCUSSION}

With the current economic cutbacks, the supporting role of the Municipal Health Services (MHS) in the adoption, implementation, and continuation of health promotion programs by teachers in schools is no longer obvious. This article provides insight into what the MHS still does and intends to do around supporting the implementation process of a sex education program, Long Live Love (LLL), and the factors that affect this.

The results show that increasingly fewer MHS-es are involved in supporting LLL. Despite budget cuts and other barriers, the MHS still wants to continue supporting the implementation process of the new LLL in schools in the future. The MHS thus remains an important supporting party for health promotion programs in schools. The future support which they can provide around the implementation of the new LLL curriculum, will, however, be limited to the promotion of dissemination and adoption. The barriers 
and enabling factors that will influence (and have influenced) the supporting role of the MHS can be attributed to the LLL curriculum, and the role of teachers, schools, MHS and municipalities.

The curriculum must be evidence-based and current. In addition, there should be regular updates so the MHS-es have something new to report to the teachers. Offering part of the program online will facilitate the uploading of updates.

Teachers must recognize the importance of the theme of sexuality to adopt LLL and have the skills to (dare) to discuss the theme of sexuality, in order to be able to deal with difficult situations in the classroom, and to schedule time to implement LLL well. The importance can be communicated by the MHS through interpersonal contact with teachers and the provision of information leaflets or teacher training. The MHS would like to be supported in this through the provision of promotional materials and by receiving a training. Since the MHS will be less able to carry out activities in the implementation and continuation stages, support for teachers in that area is desired, which is independent of the MHS. This would be possible by offering teachers coaching via a website, including tips, for example, on discussing the theme and examples of how other teachers deal with difficult situations.

A lack of structural embedding of relational and sex education in schools will limit implementation of programs such as LLL. Structural embedding at the school level can be promoted by allowing program goals to overlap with the school curriculum and by inclusion of sexual health in core educational objectives.

The lack of financial resources and collaboration between the various departments within the MHS, as well as relational and sexual health being a low priority within the organization, is an obstacle to the supporting role which the MHS can offer for such health-promoting school programs. This problem is probably partly due to comparable budget cuts and / or a lack of attention for relational and sexual health within the municipalities. In both cases, an intervention at the administrative level seems necessary to prioritize sexual health so that money is made available and internal collaboration can be improved within the MHS. This allows the creation of support for adoption and implementation of programs such as LLL. The results of this study are also relevant for other health promoting interventions in schools that need to be externally supported, because the challenges in the school-setting are similar. 


\section{Limitations and strengths}

This research has self-reported data and MHS professionals have had to make some estimates for a new curriculum that did not exist yet at the time of this research. In addition, it is possible that mainly MHS professionals who were more optimistic about LLL decided to participate in the study. This may have led to a more positive picture than would be found among those who did not respond. The results of this study are probably fairly representative of the Netherlands since most of the MHS-es and regions were represented. The qualitative and quantitative data reinforce one another.

\section{CONCLUSION}

Despite the personal involvement of MHS professionals on the topic of sexual health, their role around the implementation of LLL is limited by budget cuts and various barriers on the level of MHS, schools and municipalities. The MHS professional, however, remains an important intermediary for optimizing the implementation process of LLL and other school-based health promotion programs. Only once the MHS adopt and implement their activities to promote a curriculum, will the implementation process run smoothly among teachers. Now that the barriers and facilitating factors that the MHS is facing in a time of economic crisis have been identified, it offers opportunities to bring about change and strengthen capacities. A broad comprehensive intervention seems partly necessary at the level of municipalities, municipal health services, and schools. Additionally, MHS support should be provided by delivering good promotional and educational materials, and training. Finally, we must look at alternative methods to support teachers in the implementation and continuation of school health programs such as online coaching, since the MHS will primarily be deployed to stimulate dissemination and adoption. 


\section{Notes ${ }^{*}$}

\section{a. Attainment / Core objectives}

Core objectives of education are targets for education, established by the Dutch Ministry of Education. The core objectives provide guidelines and minimum requirements for education and the level of knowledge and skills which students acquire. Schools choose their own method to achieve these core objectives. Sexuality and sexual diversity have been included in the core objectives of secondary education since December 2012.

\section{b. Health promoting school approach}

This is a demand-oriented method of working that supports schools to structurally work with health and safety.

\section{c. Municipal public health policy}

The Public Health Act (WPG) requires that each municipality develops a policy that includes ambitions, choices and priorities in the field of public health and collective prevention.

\section{d. Teaching material toolkit}

The documentation center within the MHS that lends toolkits to schools with various teaching materials for different health themes, including the LLL curriculum for sexual health. 
CHAPTER 4

\title{
Innovatively supporting teachers'
}

\section{implementation of school-based sex \\ education: Developing a Web-based}

\author{
coaching intervention from
}

problem to solution

This chapter is published as:

Schutte, L., van den Borne, M., Kok, G., Meijer, S., Mevissen, F.E.F. (2016). Innovatively supporting teachers' implementation of school-based sex education: Developing a

Web-based coaching intervention from problem to solution. Journal of Medical Internet Research, 18 (7), e136. PMID: 27405241 


\section{ABSTRACT}

Background: Full program implementation is crucial for effectiveness but is often overlooked or insufficiently considered during development of behavioral change interventions. For school-based health promotion programs, teachers are key players in program implementation but teacher support in this phase is mostly limited to technical support and information. To ensure optimal implementation of the Dutch school-based sexual health program Long Live Love, an online coaching website was developed to support teachers in complete and fidel program implementation.

Objective: The aim of this paper is to provide insight into the process of systematic development of a web-based coaching intervention to support teachers in their implementation of a school-based sexual health program.

Methods: The Intervention Mapping (IM) protocol was applied for the development of a theory- and evidence-based intervention. The IM process begins with (1) a needs assessment, followed by (2) the formulation of change objectives, (3) the selection of theory-based intervention methods and practical applications that take the parameters for effectiveness into consideration, (4) integration of practical applications into an organized program, (5) planning for adoption, implementation and sustainability of the program, and finally, (6) generating an evaluation plan to measure program effectiveness.

Results: Teacher's implementation behavior was characterized by inconsistently selecting parts of the program and not delivering (all) lessons as intended by program developers. Teachers, however, did not perceive this behavior as problematic, revealing the discrepancy between teacher's actual and perceived need for support in delivering Long Live Love lessons with completeness and fidelity. Teachers did, however, acknowledge different difficulties they encountered which could potentially negatively influence the quality of implementation. With the IM protocol, this online coaching intervention was developed based on a concept of unobtrusive coaching, by and for teachers, to bring about change in teachers' implementation behavior.

Conclusions: This paper provides an example of an online intervention to bring about behavioral change in a target group of intermediaries who lack intrinsic motivation for coaching and who's perceptions differ from their actual problematic behavior. The IM protocol is a useful tool for guiding the scientific development of interventions and making them compatible with the needs and preferences of the target group. 


\section{INTRODUCTION}

Schools provide the ideal setting to reach youngsters with health promotion programs. Although decisions to use programs in schools are typically made at the administrative level, teachers are the primary agents of school-based prevention efforts. Their support, motivation, and commitment is crucial to implementation success (Hunter et al., 2001). In the Netherlands, teachers are the ones who decide to use a school-based program in their classroom (adoption), deliver the program to students (implementation) and continue to do so in the long run (continuation) (Paulussen et al., 1995). Many evidencebased programs consider the implementers of the programs, such as teachers, as "core" to the success of the program (Coyle et al., 2011).

Implementation of school-based health promotion programs is, however, not optimal (Bessems et al., 2014; Peters et al., 2009; Schutte et al., 2014). These programs are not implemented with sufficient strength and fidelity to produce measurable outcomes (Gottfredson \& Gottfredson, 2002). A monitoring of school-based interventions in the Netherlands showed that only 5-10\% of teachers who have bought a program, implement it fully in accordance with the ideas of the program designer, resulting in reduced program effectiveness (Hoekstra et al., 2009). The behavior of program implementers is often an aspect that is overlooked or insufficiently considered in program development. There is a need for greater attention for quality of implementation (Fagan \& Mihalic, 2003).

Implementation is a process consisting of several phases, namely adoption, implementation, and continuation (Rogers, 1995). Teachers need support in every phase of the implementation process to enable them to effectively carry out the program in their lessons (Durlak \& DuPre, 2008; Rohrbach, Gunning, Sun, \& Sussman, 2010). Most interventions were aimed at supporting teachers in the awareness and adoption of the program but little is known or created to support teachers in the implementation phase (Bessems et al., 2014; Domitrovich \& Greenberg, 2000; Durlak, 1998; Fagan \& Mihalic, 2003). Support in this phase is crucial, however, for optimal program effectiveness (Domitrovich \& Greenberg, 2000).

Especially when it comes to providing school-based sex education, delivering such lessons is not a simple or obvious task; teachers, who are key to the success of such programs, not only require knowledge and a positive attitude but also certain skills and competencies to deliver a range of sensitive topics in these lessons, and to deal with the difficulties encountered during implementation of the program. To prepare teachers for program use, specialized and effective training is necessary (Ahmed et al., 2006). 
Although training often equips teachers with skills for correct implementation, it is not enough (Dusenbury et al., 2003). It remains important to provide teachers with more personal assistance and ongoing support, and consultation during program delivery to ensure the quality of implementation (Schutte et al., 2014, Durlak, 1998; Kramer et al., 2000; Rohrbach et al., 1993). This support needs to be of sufficient duration to achieve depth in teachers' skills and behavioral change throughout program delivery (Han \& Weiss, 2005). Paulussen et al (1995) highlighted the importance of providing support prior to and during the implementation of a curriculum by way of training and technical and didactic assistance to ensure enduring success.

\section{The 'Long Live Love' program}

In the Netherlands, Long Live Love (LLL) is the most widely used, effective schoolbased sex education program, partly due to a successful implementation strategy (Hofstetter et al., 2014). An earlier study on the implementation of LLL revealed that trainings from an external party, the Municipal Health Services (MHS), resulted in improved implementation of LLL by teachers (Bos, de Jongh, \& Paulussen, 2010). Due to economic cutbacks, the supportive role of the Dutch MHS has recently been limited to predominantly stimulating dissemination and adoption of LLL and preparing teachers for initial implementation. They lack the capacity to provide intensive and longterm support (Schutte et al., 2014). Additionally, MHS professionals lack the didactic expertise and skills to be appropriate role models for teachers in teaching skills for adequate implementation (Bos et al., 2010). Teachers therefore need another form of support during implementation to compensate for the limitations of the MHS and to complement the existing implementation strategy of LLL.

To contribute to the limited documentation of implementation interventions, this article presents the systematic development of an online coaching intervention, Lesgevenindeliefde.nl (Teaching Love). The website is part of a broader implementation strategy and supports teachers in implementation of the school-based sex education program, Long Live Love (LLL). The online coaching intervention aims at an optimal implementation, with completeness and fidelity, of LLL by teachers. As of date, no other web-based coaching website to support teachers in delivering school-based sex education is known in the Netherlands (Marino, 2004; Zwaneveld \& Rigter, 2009). This paper will provide insight into teacher implementation of a school-based sex education program, LLL, and describe the complete cycle of development of this coaching website, from problem to solution. The website is developed applying Intervention Mapping (IM), a protocol to systematically develop interventions using theory and empirical 
evidence (Bartholemew et al., 2011). IM has proven to be an effective protocol in the development of various online health promotion interventions (Makai et al., 2014; van Bruinessen et al., 2014; Voogt, Poelen, Kleinjan, Lemmers, \& Engels, 2014).

\section{METHOD}

\section{Developing Effective Behavior Change Interventions}

Intervention Mapping (IM) is a protocol for the development of theory- and evidencebased interventions. It maps the path from identification of a problem to the development of a solution. Although IM is presented as a series of six steps (see Figure 1) it is an iterative and cumulative process in which, respectively, the developer moves back and forth between the steps and in which each step is based on the outcomes of the previous ones (Bartholemew et al., 2011). The six steps are (1) conduct a needs assessment, (2) create matrices of change objectives, (3) select theory-based methods and practical applications, (4) organize methods and applications into an intervention program, (5) plan for adoption, implementation and sustainability of the program and (6) generate an evaluation plan (Bartholemew et al., 2011).

\section{Intervention Mapping Steps}

The first step, the Needs Assessment, begins with establishing a participatory planning group, represented by potential program participants and implementers. This step consists of a full analysis and description of the problem through an epidemiologic, behavioral and social analysis of the at-risk-group. By means of qualitative and/or quantitative research, behaviors and environmental factors related to the health problem are identified.

In step 2 a transition is made from the problem to the solution, namely specifying what should change to prevent or to minimize a problem. Step 2 begins with the formulation of the behavioral and environmental outcomes to be achieved by the intervention followed by a breakdown of these outcomes into specific sub-behaviors called performance objectives, stating what the target group needs to do to achieve these desired outcomes. Next, determinants are selected that are linked to these objectives. Finally, these determinants and performance objectives are connected in a matrix to create change objectives, which state the specific goals to be achieved as a result of the intervention. 


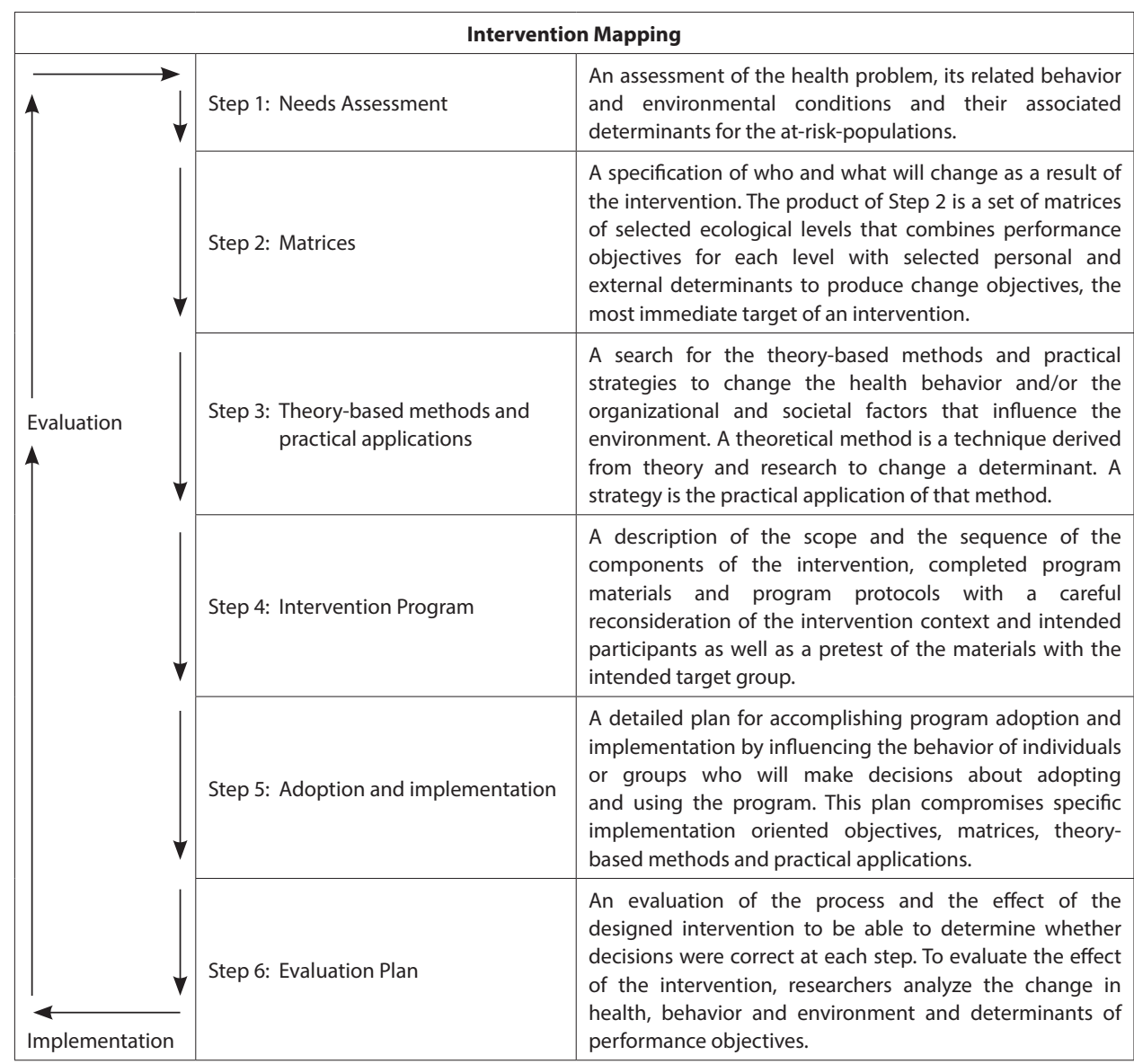

Figure 1. Intervention Mapping process. Adapted from Bartholemew et al., 2011.

In step 3 theoretical methods are selected that change the specified determinants and achieve the change objectives. A method is a general technique for influencing change in determinants. These methods are translated into practical applications while taking the parameters for use into consideration. These parameters provide conditions under which effectiveness of the application is ensured. The applications should fit within the context of the intervention and the target group.

In step 4, creative and effective program components and materials are developed based on the previous steps. The challenge is to cover all selected theoretical methods, practical applications and change objectives. The end product of this step is a coherent program that remains true to the planning that has been accomplished in step 1, 2 and 3. 
Effective programs, however, will have limited impact if they are never, incorrectly or incompletely used. An appropriate adoption, implementation and continuation plan is essential to achieve the desired outcomes. The main goal of step 5 is to ensure that the intervention will be used and maintained over time for as long as is needed. To realize this goal the developer must complete the same tasks as in step 1,2, 3 and 4, resulting in an effective intervention plan for optimal adoption, implementation and continuation of the intervention.

In the final step of the iterative and cumulative IM process, the effect and implementation of the intervention are evaluated. An evaluation gives insight into the extent to which the earlier formulated goals are achieved after application of the intervention. The evaluation is divided in outcomes of quality of life, health and behavior. A process evaluation is necessary to understand these outcomes and gives insight in the 'black box' underlying the effect. The 'black box' provides insight into what happens between application of the intervention and the outcomes. This paper presents outcomes of steps 1 to 5 . The effect and process evaluation will be presented in a separate paper.

\section{RESULTS}

\section{IM Step 1: Needs assessment}

At the start of the project, a participatory planning group was set up, consisting of a panel of health promotion professionals $(\mathrm{N}=10)$, teachers $(\mathrm{N}=4)$ and $\mathrm{MHS}$ professionals $(\mathrm{N}=2)$. The goal for the selected group was to think along in the intervention development process and be consulted throughout the project. The needs assessment was conducted by means of (1) analyzing existing programs and reviewing the literature and (2) qualitative research.

\section{Analyzing Existing Programs and Literature Study}

The search for existing programs in the Netherlands did not reveal the existence of systematically developed and evidence-based online interventions for coaching teachers in providing Sexual Reproductive Health (SRH) lessons. The search did result in a few materials to support teachers in teaching SRH. This support was, however, minimal and was not aimed at coaching to bring about behavioral change. In the field of sexual health promotion, for example, there is a website for teachers but this is limited to providing materials and practical information on how to provide such lessons without further coaching (Van de Bongardt, Mouthaan, \& Bos, 2009). This is insufficient for behavioral change, which is necessary for fidel and complete program delivery (Bartholemew et al., 2011; Hansen et al., 2009; Rohrbach et al., 2010). 
The literature study revealed that limited examples are available on the development, execution and evaluation of implementation enhancing interventions in general. In fields other than $\mathrm{SRH}$, studies were also mainly focused on the provision of technical support (Farmer-Dougan, Viechtbauer, \& French, 1999; Hesselink, van der Sluis, \& Martens, 2011; Jones, Wickstrom, \& Friman, 1997; Kam, Greenberg, \& Walls., 2003; Mihalic, Fagan, \& Argamaso, 2008; Mortenson \& Witt, 1998; Noell, Witt, Gilbertson, Ranier, \& Freeland, 1997; Witt, Noell, LaFleur, \& Mortenson, 1997). These studies, however, were not aimed at coaching to bring about behavioral change. Although related to themes other than SRH, these studies reconfirm the limited existence of evidence-based coaching interventions and emphasize the importance of systematically developing an intervention to accomplish sustainable behavioral change. Supporting teachers during implementation will enable them to deliver the lessons as complete as possible (completeness) and according to previously formulated program goals (fidelity) for optimal effectiveness (Durlak \& DuPre, 2008; Rohrbach et al., 2010).

\section{Qualitative research}

Qualitative data were collected by conducting semi-structured interviews with teachers to provide more insight into their implementation behavior and to get insight in their (perceived) need and preferences for coaching. A sample of 15 teachers from 12 different schools was selected from the database of schools who had bought the previous LLL program. The selection was made based on regional representation and gender. Teachers were asked to participate in the research by e-mail. Furthermore, during the interview process, the snowball effect resulted in the involvement of three additional teacher respondents. The main reason for non-response was a lack of time. In the end, $\mathrm{N}$ $=11$ teachers signed up to participate for the interviews.

A topic list guided the interviews with 11 teachers ( 5 male, 6 female) from nine different schools and regions, with diverse levels of experience in teaching SRH. The average duration of the interviews was approximately 40 minutes. See Textbox 1 for the topic list. This topic list was derived from a conceptual model based on Theory of Planned Behavior (TPB) (Ajzen, 2011), the Social Cognitive Theory (SCT) (Bandura, 1986) and the Diffusion of Innovations theory (Dol) (Rogers, 1995). These theories are often used to explain implementation behavior of teachers (Wiefferink et al., 2005; Paulussen et al., 1995). 
Textbox 1. Topic list Needs Assessment Lesgevenindeliefde.nl

- What do you do with the theme of $\mathrm{SRH}$ ?

- How do you teach your SRH lessons?

- Do you have any idea how other colleagues are dealing with the theme of SRH?

- Do you have any idea how less experienced colleagues are teaching SRH?

- Which difficulties do you experience in teaching $\mathrm{SRH}$ ?

- How do you deal with those difficulties?

- How can you address those difficulties?

- What do you need to be able to teach SRH optimally?

- What do you need to be able to effectively teach Long Live Love (LLL)?

- How do you prepare your SRH - lessons or for teaching the Long Live Love program?

- Do you use any kind of support or a program during the application of the LLL program or the SRH lessons?

- What do you do in the evaluation of the SRH-lessons or the Long Live Love program?

- Which support would you like to receive in teaching $\mathrm{SRH}$ ?

- Which support would other, and maybe less experienced, colleagues like to receive in teaching $\mathrm{SRH}$ ?

- How should this support or coaching look like?

- How can this be implemented in an internet based coaching program?

The interviews revealed that teacher implementation of SRH programs, including Long Live Love, is not optimal; various components of the program are selected and delivered, rather than completing the entire program and implementing it as intended by the program developers. Teachers describe their implementation behavior as making a selection of program components, adjusting the program with their own additions, not delivering the program in its entirety, limited use of the teacher manual and a lack of planning, preparation and evaluation. This suboptimal implementation behavior may lead to reduced program effectiveness (Bessems et al., 2014; Peters et al., 2009).

T: "When we teach Long Live Love, we sometimes make our own additions and modifications. The program lacks practical assignments. It's mainly about reading and answering questions."

\section{I: "What is required to provide SRH programs optimally?"}

T: "More practical materials. Actually, teaching SRH is mainly reading and answering questions. We do improvise with other materials because the SRH program alone does not contain sufficient practical assignments." 
Teachers do not acknowledge their behavior as problematic; they do not see the importance of delivering the lessons with completeness and fidelity for achieving program effectiveness and therefore expressed minimal need for coaching. Although the perceived need for support in implementation was low, teachers did recognize several difficulties that may be encountered, especially - according to them - by less experienced colleagues, during the provision of SRH lessons.

T: "Some colleagues, not myself of course, experience difficulties in talking about sexuality. How do you begin? How are you going to talk about it or cope with it? Coaching could be given for those kinds of problems."

An inability to adequately deal with these difficulties can negatively interfere with optimal implementation of SRH programs. According to the respondents, teachers providing SRH lessons may encounter the following difficulties: (1) Barriers to creating a safe and trusted atmosphere in the classroom (2) Feelings of shame or a closed attitude towards sexuality (3) Dealing with personal questions asked to them or to other students (4) Coping with individual student problems related to SRH (5) Dealing with homo negative reactions and behavior of students in the classroom (6) Anticipating on negative events on social media and internet among students in relation to $\mathrm{SRH}$ (7) Providing SRH lessons in a culturally-', gender- and sexually divers classroom.

I: "What skills, knowledge or other factors do you need to educate the students about SRH?"

T: "You have to perceive the world as students do. You shouldn't be surprised by comments in the classroom. You shouldn't assume that they are not sexually active. But you also need skills to create a safe and secure atmosphere. For the students. They also have to be able to talk freely about their experiences. That you can use these experiences to give information and to integrate this in the lessons. It is also really important that there is respect for each other and for each other's boundaries. That you are able to establish your boundaries. That you can be different. That's also an important focus of our lessons."

When teachers were asked what was necessary for effective implementation of SRH programs, teachers mentioned a desire for materials they could use in the classroom with their students. They were not focused on their own quality of implementation but instead they were on the lookout for practical tools to use during lessons. 
T: "Teachers don't often place their problems on a forum after a lesson. They will use it to find ideas for their lessons. Then they search tediously. But they won't share the experiences they had during a lesson on a forum."

I: "How can we optimally coach a teacher so that he is capable of teaching Long Live Love or SRH?"

T: "For teachers, it is necessary to be well informed about the topic. To have adequate, sufficient and reliable information available. That they have the feeling; 'I can answer questions.' That's important in my opinion."

I: "What more do teachers need?"

T: "Materials and good information. Ehm... Something to visualize. The classical cucumber with a condom."

When the teachers were directly asked about their need for coaching, the respondents answered to be satisfied with their own teaching method and expressed minimal need to be coached. They felt they could prepare sufficiently by reviewing the program materials and the teacher manual individually or were incidentally assisted by other teachers in preparations for program implementation.

T: "Well, in my case, I don't know if I would use it (an e-coaching website) extensively. Because I have been teaching this (SRH) for a long time, I know a lot and everything can be talked about. If I don't know something, I go to a colleague. So, I don't know if I would make use of it. I would take a look. Purely out of curiosity. Maybe I am too arrogant but I really can't think of anything I would need help for."

Critical reflection of one's own behavior and working on professional development, are necessary for creating awareness and establishing sustainable behavioral change (Parsloe \& Leedham, 2009). Teachers do not seem to see the connection between completeness, fidelity and program effectiveness, do not see their sub-optimal implementation behavior as problematic or are not aware of potentially challenging situations and therefore do not see a need for behavioral change and coaching. Teachers need to be aware of the importance of completeness and fidelity in relation to program effectiveness, have insight in their (suboptimal) implementation behavior and be aware of potentially challenging situations, to ultimately improve completeness and fidelity of program delivery. Critical self-reflection leads to awareness of own behavior 
and is the first step of coaching teachers towards behavioral change and professional development. Without a genuine recognition of need and desire, it is almost impossible to change behavior (Parsloe \& Leedham, 2009).

I: "Do you think teachers would make use of such a coaching website?"

T: "If you point out the things that can go wrong, they have to prepare to deal with them. If you can trigger that, you've already come a long way. Teachers will start to reflect; "how does that affect me?"

If a coaching intervention was to be developed, it is important, according to the teachers, to develop an intervention that is easy to use and accessible and does not cost a lot of time and effort because teachers claimed that they only have limited time and resources to prepare or to evaluate the lessons.

I: "How should such an e-coaching website look like?"

T: "There shouldn't be any complicated access codes. A lot of people drop out if they see that. It should be easily accessible. It shouldn't cost me an hour and a half to browse. I don't have time for that. Ideally you can select various components on a website while browsing; difficult situations that you may encounter. If a recognizable situation is described by a fellow teacher, I might think, this can happen to me as well."

The possibility of developing virtual coaching, in which teachers are guided throughout the implementation process by a virtual buddy, was discouraged by most of the respondents. Instead of a virtual buddy, teachers expressed the preference to communicate with colleagues within different schools to exchange ideas and teaching methods or to solve problems they encounter during the provision of SRH lessons.

I: "Do you evaluate or discuss your lessons?"

T: "No, it is a very lonely profession... It is progressive thinking to learn from other teachers."

In conclusion, the needs assessment revealed an interesting finding: there is a discrepancy between teachers perceived and actual need for support in providing $\mathrm{SRH}$ lessons effectively. Teachers do not perceive their implementation behavior as problematic but their actual implementation behavior does not fulfil the required completeness and fidelity for program effectiveness. To ensure fidelity 
and completeness of program implementation, it remains important to first create awareness, by means of self-reflection, of (1) the importance of completeness and fidelity in program implementation, (2) teachers' current implementation behavior, and (3) the difficult situations they could potentially encounter. To achieve behavioral change, and contribute to professional development, teachers should subsequently be supported in dealing with the common difficulties mentioned and be provided with the knowledge and skills they need to implement SRH programs effectively. A careful choice for unobtrusive coaching techniques was made to ultimately bridge the gap between the perceived need and actual need of teachers for coaching. The technique of unobtrusive coaching is required to create awareness and accomplish behavioral change, despite teacher's resistance to coaching and ultimately optimize the role of the teacher in providing high quality SRH lessons.

\section{IM step 2: Matrices of change objectives}

Based on the needs assessment and literature review, a program goal was formulated and subdivided into four desired behavioral outcomes for teachers. The program goal was that teachers in all secondary schools in the Netherlands implement Long Live Love completely and according to its formulated goals (fidelity). The behaviors associated with this program goal were that teachers (1) reflect critically on and become aware of their implementation behavior regarding SRH, (2) implement LLL completely, (3) implement LLL according to guidelines in the teacher manual and, (4) deal adequately with difficulties that may be encountered during provision of SRH. These behaviors formed the outcomes of the intervention and were subsequently broken down into performance objectives. Performance objectives specified what teachers needed to do to perform those desired behaviors. The formulated behavioral outcomes and related performance objectives are presented in Table 1.

Performance objectives were then linked with their associated personal and external determinants. Determinants were specified based on the results of the needs assessment, a literature review and a review of theories. Social influence was not selected as a determinant because the interviews revealed that teachers individually determined their own method of teaching. However, skills, self-efficacy, attitude, and knowledge were found to be important determinants for teachers' implementation behavior (Schutte et al., 2014). These determinants were evaluated on importance (how strongly is the determinant related to teacher's performance objectives), and changeability (how easily can the determinant be influenced by a theory-based method) which formed the basis for the final selection of determinants that the intervention would target. A matrix was then created by combining the performance objectives 
and associated determinants to create change objectives; specific and measurable goals specifying what will change among teachers as a result of the intervention. For example, 'teachers express confidence (determinant: self-efficacy) in creating a safe and secure atmosphere in the classroom when delivering LLL (performance objective)'. See Table 2 for a selection of change objectives.

Table 1. Behavioral outcomes and performance objectives of teacher implementation.

\begin{tabular}{|c|c|c|c|}
\hline \multicolumn{2}{|c|}{ Behavioral outcomes of teachers } & \multicolumn{2}{|c|}{ Performance objectives } \\
\hline \multirow[t]{2}{*}{ B.0.1. } & $\begin{array}{l}\text { Teachers reflect and improve on their } \\
\text { implementation behavior regarding }\end{array}$ & P.O.1. & $\begin{array}{l}\text { Teachers reflect critically on their implementation } \\
\text { behavior regarding SRH }\end{array}$ \\
\hline & $\mathrm{SRH}$ & P.O.2. & $\begin{array}{l}\text { Teachers self-monitor and improve the weaknesses in their } \\
\text { implementation behavior regarding SRH }\end{array}$ \\
\hline \multirow[t]{3}{*}{ B.O.2. } & Teachers deliver LLL to students & P.O.2.1. & Teachers cover all six lessons of LLL \\
\hline & $\begin{array}{l}\text { completely (completeness }=80 \% \text { of } \\
\text { program) }\end{array}$ & P.0.2.2. & $\begin{array}{l}\text { Teachers use all program materials of LLL in each lesson } \\
\text { (DVD, magazine, teacher manual, worksheets) }\end{array}$ \\
\hline & & P.0.2.3. & $\begin{array}{l}\text { Teacher covers the most important components of each } \\
\text { lesson, as indicated in the teacher manual }\end{array}$ \\
\hline \multirow[t]{2}{*}{ B.0.3. } & $\begin{array}{l}\text { Teachers deliver LLL to students } \\
\text { according to the guidelines in the }\end{array}$ & P.0.3.1. & $\begin{array}{l}\text { Teachers read the teacher manual as preparation for each } \\
\text { lesson }\end{array}$ \\
\hline & teacher manual (fidelity) & P.0.3.2. & $\begin{array}{l}\text { Teachers deliver each LLL lesson to students according to } \\
\text { the teacher manual }\end{array}$ \\
\hline \multirow[t]{10}{*}{ B.0.4. } & $\begin{array}{l}\text { Teachers deal adequately with the most } \\
\text { common difficulties that arise during } \\
\text { implementation of SRH }\end{array}$ & P.0.4.1. & $\begin{array}{l}\text { Teachers create a safe and trusted atmosphere in the } \\
\text { classroom during all LLL lessons whereby students feel } \\
\text { comfortable in the classroom and asking questions }\end{array}$ \\
\hline & & P.O.4.2. & $\begin{array}{l}\text { Teachers teach all themes in LLL without shame or taboos } \\
\text { interfering with the quality of the lesson }\end{array}$ \\
\hline & & P.0.4.3. & $\begin{array}{l}\text { Teachers handle personal questions of students addressed } \\
\text { to themselves depending on their personal need to } \\
\text { answer these questions }\end{array}$ \\
\hline & & P.O.4.3.1 & $\begin{array}{l}\text { Teachers intervene whenever students ask them or fellow } \\
\text { students questions that are too personal }\end{array}$ \\
\hline & & P.O.4.4. & $\begin{array}{l}\text { Teachers integrate the theme of homosexuality as self- } \\
\text { evident during all lessons of LLL }\end{array}$ \\
\hline & & P.O.4.4.1 & $\begin{array}{l}\text { Teachers intervene on homo-negative behavior of } \\
\text { students }\end{array}$ \\
\hline & & P.0.4.5. & $\begin{array}{l}\text { Teacher handle cultural-, gender- and sexual- experience } \\
\text { diversity in the classroom using an approach that address } \\
\text { and involves all students }\end{array}$ \\
\hline & & P.O.4.6. & $\begin{array}{l}\text { Teachers identify individual problems of students with and } \\
\text { refer them to the appropriate help }\end{array}$ \\
\hline & & P.O.4.7. & $\begin{array}{l}\text { Teachers address actual themes within social media and } \\
\text { internet in relation to SRH during the provision of LLL }\end{array}$ \\
\hline & & P.0.4.8. & $\begin{array}{l}\text { Teachers facilitate discussions in the classroom about } \\
\text { relationships and sexuality according to the formulated } \\
\text { goals and suggestions in the teacher manual }\end{array}$ \\
\hline
\end{tabular}


Table 2. Sample of change objectives.

\begin{tabular}{|c|c|c|c|c|}
\hline Homosexuality & Knowledge & Attitude & Self-efficacy & Skills \\
\hline \multirow[t]{5}{*}{$\begin{array}{l}\text { 1. The teacher } \\
\text { integrates the theme } \\
\text { of homosexuality as } \\
\text { self-evident during } \\
\text { all lessons of Long } \\
\text { Live Love }\end{array}$} & $\begin{array}{l}\text { K } 1.1 \text { The teacher } \\
\text { describes how } \\
\text { homosexuality is } \\
\text { integrated in the } \\
\text { lessons of Long Live } \\
\text { Love. }\end{array}$ & $\begin{array}{l}\text { A } 1.1 \text { The teacher } \\
\text { expresses the } \\
\text { importance of a } \\
\text { positive attitude of } \\
\text { a teacher towards } \\
\text { homosexuality during } \\
\text { the application of the } \\
\text { lessons of Long Live } \\
\text { Love. }\end{array}$ & $\begin{array}{l}\text { SE } 1.1 \text { The teacher } \\
\text { expresses confidence } \\
\text { in ability to replace } \\
\text { 'he' and 'she' by 'he' } \\
\text { and 'he' or 'she' and } \\
\text { 'she'. }\end{array}$ & $\begin{array}{l}\text { S } 1.1 \text { The teacher } \\
\text { demonstrates how } \\
\text { he/she continually } \\
\text { integrates the theme } \\
\text { of homosexuality in } \\
\text { the lessons. }\end{array}$ \\
\hline & $\begin{array}{l}\text { K } 1.2 \text { The teacher } \\
\text { lists the moments } \\
\text { in the Long Live } \\
\text { Love lessons where } \\
\text { the theme of } \\
\text { homosexuality can } \\
\text { be discussed as a } \\
\text { self-evident part of } \\
\text { the lesson. }\end{array}$ & $\begin{array}{l}\text { A } 1.2 \text { The teacher } \\
\text { expresses the } \\
\text { advantages } \\
\text { of integrating } \\
\text { homosexuality as } \\
\text { self-evident during } \\
\text { the application of } \\
\text { Long Live Love. }\end{array}$ & $\begin{array}{l}\text { SE } 1.2 \text { The teacher } \\
\text { expresses confidence } \\
\text { in the ability to } \\
\text { continually integrate } \\
\text { homosexuality and } \\
\text { certainly not avoid } \\
\text { the theme in the } \\
\text { lessons of Long } \\
\text { Live Love in case of } \\
\text { negative reactions } \\
\text { from students. }\end{array}$ & $\begin{array}{l}\text { S } 1.2 \text { The teacher } \\
\text { demonstrates skill to } \\
\text { not avoid the theme } \\
\text { of homosexuality } \\
\text { despite possible } \\
\text { adverse or negative } \\
\text { reactions from } \\
\text { students. }\end{array}$ \\
\hline & $\begin{array}{l}\text { K } 1.3 \text { The teacher } \\
\text { describes how he/ } \\
\text { she plans to integrate } \\
\text { homosexuality in the } \\
\text { lessons of Long Live } \\
\text { Love. }\end{array}$ & $\begin{array}{l}\text { A } 1.3 \text { The teacher } \\
\text { expresses the } \\
\text { importance of } \\
\text { mentioning 'he' } \\
\text { and 'he' or 'she' and } \\
\text { 'she' instead of 'he' } \\
\text { and 'she' during the } \\
\text { lessons of Long Live } \\
\text { Love. }\end{array}$ & $\begin{array}{l}\text { SE } 1.3 \text { The teacher } \\
\text { expresses confidence } \\
\text { in the ability to } \\
\text { protect students } \\
\text { with feelings of } \\
\text { homosexuality } \\
\text { against a feeling of } \\
\text { discomfort or social } \\
\text { pressure. }\end{array}$ & $\begin{array}{l}\text { S } 1.3 \text { The teacher } \\
\text { demonstrates skills } \\
\text { to stimulate the } \\
\text { discussions about } \\
\text { homosexuality } \\
\text { in which respect } \\
\text { and acceptance } \\
\text { are important key } \\
\text { aspects in this in- } \\
\text { depth discussion. }\end{array}$ \\
\hline & $\begin{array}{l}\text { K } 1.4 \text { The teacher } \\
\text { explains that when } \\
\text { 'he' and 'she' is } \\
\text { mentioned this can } \\
\text { also be replaced by } \\
\text { 'he' and 'he' or 'she' } \\
\text { and 'she'. }\end{array}$ & $\begin{array}{l}\text { A } 1.4 \text { The teacher } \\
\text { expresses the } \\
\text { importance of } \\
\text { discussing and } \\
\text { integrating the theme } \\
\text { of homosexuality, } \\
\text { especially when } \\
\text { the students react } \\
\text { negatively. }\end{array}$ & $\begin{array}{l}\text { SE } 1.4 \text { The teacher } \\
\text { expresses confidence } \\
\text { in the ability to } \\
\text { communicate } \\
\text { the message that } \\
\text { homosexuality is not } \\
\text { limited to borders, } \\
\text { cultures or countries } \\
\text { during the lessons. }\end{array}$ & $\begin{array}{l}\text { S } 1.4 \text { The teacher } \\
\text { demonstrates how } \\
\text { he/she protects } \\
\text { students with } \\
\text { homosexual feelings } \\
\text { from a feeling of } \\
\text { discomfort. }\end{array}$ \\
\hline & $\begin{array}{l}\text { K } 1.5 \text { The teacher } \\
\text { explains the reasons } \\
\text { why he or she will } \\
\text { strive towards a self- } \\
\text { evident integration } \\
\text { of homosexuality as } \\
\text { theme in the lessons } \\
\text { of Long Live Love. }\end{array}$ & $\begin{array}{l}\text { A } 1.5 \text { The teacher } \\
\text { describes the } \\
\text { importance of } \\
\text { effectively coping } \\
\text { with feelings } \\
\text { of pressure or } \\
\text { discomfort of } \\
\text { students with feelings } \\
\text { of homosexuality } \\
\text { during the lessons of } \\
\text { Long Live Love. }\end{array}$ & $\begin{array}{l}\text { SE } 1.5 \text { The teacher } \\
\text { expresses confidence } \\
\text { in the ability to be } \\
\text { continually alert of } \\
\text { possible individual } \\
\text { confrontations } \\
\text { between students } \\
\text { about homosexuality. }\end{array}$ & \\
\hline
\end{tabular}


Table 2. (continued)

\begin{tabular}{|c|c|c|c|c|}
\hline Homosexuality & Knowledge & Attitude & Self-efficacy & Skills \\
\hline & & $\begin{array}{l}\text { A } 1.6 \text { The teacher } \\
\text { expresses the } \\
\text { importance of } \\
\text { informing students } \\
\text { that homosexuality } \\
\text { is not limited to a } \\
\text { culture, to borders or } \\
\text { to time periods. }\end{array}$ & & \\
\hline \multirow[t]{3}{*}{$\begin{array}{l}\text { 2. Teachers intervene } \\
\text { on homo-negative } \\
\text { behavior of students }\end{array}$} & $\begin{array}{l}\text { K } 2.1 \text { The teacher } \\
\text { lists the signs he/she } \\
\text { should be aware of } \\
\text { which could indicate } \\
\text { homonegative ideas } \\
\text { or behavior among } \\
\text { students. }\end{array}$ & $\begin{array}{l}\text { A } 2.1 \text { The teacher } \\
\text { expresses a } \\
\text { disapproving } \\
\text { attitude towards } \\
\text { homonegative } \\
\text { behavior during the } \\
\text { application of Long } \\
\text { Live Love. }\end{array}$ & $\begin{array}{l}\text { SE } 2.1 \text { The teacher } \\
\text { expresses confidence } \\
\text { in the ability to be } \\
\text { continually alert of } \\
\text { signs or behavior } \\
\text { of students in the } \\
\text { classroom which can } \\
\text { be homonegative. }\end{array}$ & $\begin{array}{l}\text { S } 2.1 \text { The teacher } \\
\text { demonstrates skills } \\
\text { to constantly be alert } \\
\text { of homonegative } \\
\text { signs or behavior of } \\
\text { students during the } \\
\text { lessons. }\end{array}$ \\
\hline & $\begin{array}{l}\text { K } 2.2 \text { The teacher } \\
\text { describes which } \\
\text { methods can be } \\
\text { used effectively } \\
\text { in the classroom } \\
\text { when students } \\
\text { have homonegative } \\
\text { ideas or show } \\
\text { homonegative } \\
\text { behaviors. }\end{array}$ & $\begin{array}{l}\text { A } 2.2 \text { The teacher } \\
\text { describes the } \\
\text { importance of being } \\
\text { constantly alert of } \\
\text { homonegative signs } \\
\text { or behavior of the } \\
\text { students. }\end{array}$ & $\begin{array}{l}\text { SE } 2.2 \text { The teacher } \\
\text { expresses confidence } \\
\text { in ability to take } \\
\text { measures when } \\
\text { students act } \\
\text { homonegatively in } \\
\text { the classroom. }\end{array}$ & $\begin{array}{l}\text { S } 2.2 \text { The teacher } \\
\text { demonstrates skills } \\
\text { to adequately deal } \\
\text { with homonegative } \\
\text { signs or behavior } \\
\text { of students in the } \\
\text { classroom. }\end{array}$ \\
\hline & $\begin{array}{l}\text { K } 2.3 \text { The teacher } \\
\text { describes how } \\
\text { homonegative } \\
\text { reactions of students } \\
\text { can be used as a } \\
\text { subject for discussion. }\end{array}$ & $\begin{array}{l}\text { A } 2.3 \text { The teacher } \\
\text { expresses the } \\
\text { importance of taking } \\
\text { timely measures } \\
\text { when students act } \\
\text { homonegatively in } \\
\text { the classroom. }\end{array}$ & & \\
\hline
\end{tabular}




\section{IM step 3: Theory-based methods and practical applications}

In this step, we selected theory-based methods to change the specified determinants and ultimately achieve the change objectives. These methods were derived from theories, predominantly the Social Cognitive Theory, Elaboration Likelihood Model, and the Trans Theoretical Model and from evidence in the empirical literature stating that the methods might have the desired effect to change the determinant (Bartholemew et al., 2011). The parameters, the conditions under which the methods were expected to be effective, were considered when translating them into practical applications, which fit within the context of the intervention and target group. Table 3 shows examples of selected theoretical methods, practical applications and their relation to the selected determinants. For example, behavioral journalism is a potentially effective method for increasing self-efficacy but will only work under the condition that authentic interviews are used with actual community members, which represent the desired message (McAlister, 1995). This method was translated into the application of role model stories where teachers share their experiences and suggestions in dealing with difficult situations. 
Table 3. Methods and applications.

\begin{tabular}{|c|c|c|c|c|}
\hline Determinants & Methods & Parameters & Applications & $\begin{array}{l}\text { How population, context and parameters } \\
\text { were taken into account }\end{array}$ \\
\hline \multirow[t]{3}{*}{ Knowledge } & Elaboration & $\begin{array}{l}\text { High motivation } \\
\text { and cognitive } \\
\text { ability, personally } \\
\text { relevant messages, } \\
\text { surprising, } \\
\text { repeated, } \\
\text { self-pacing, not } \\
\text { distracting, easily } \\
\text { understandable, } \\
\text { include direct } \\
\text { instructions }\end{array}$ & $\begin{array}{l}\text { Informative } \\
\text { texts, tips \& FAQ }\end{array}$ & $\begin{array}{l}\text { Population: The informative text was } \\
\text { derived from professional teacher channels } \\
\text { as well as from teachers themselves and } \\
\text { health promotion, didactic \& pedagogic } \\
\text { professional information. } \\
\text { Context: Texts and tips were included within } \\
\text { each sub homepage for each difficulty. } \\
\text { Parameters: The texts were revised by } \\
\text { an editor, composed based on teachers' } \\
\text { experiences and relevant literature for the } \\
\text { area of expertise. }\end{array}$ \\
\hline & Feedback & $\begin{array}{l}\text { Specific, follows } \\
\text { behavior in time, } \\
\text { individual }\end{array}$ & $\begin{array}{l}\text { E-mail-option } \\
\& \text { options to } \\
\text { post reactions } \\
\text { on role-model- } \\
\text { stories and films }\end{array}$ & $\begin{array}{l}\text { Population: To be able to answer specific } \\
\text { individual questions and provide individual } \\
\text { feedback this function was integrated in } \\
\text { the website. } \\
\text { Context: In case of a direct coaching } \\
\text { question from the visiting teacher, he/ } \\
\text { she was able to ask questions through a } \\
\text { mail-function or post comments below a } \\
\text { story or film. } \\
\text { Parameters: The mail form was only } \\
\text { accessible for the individual teacher. The } \\
\text { question or answer was not published for } \\
\text { others. Continuation in contact could be } \\
\text { initiated by the teacher. }\end{array}$ \\
\hline & Discussion & $\begin{array}{l}\text { Listening to } \\
\text { learner to ensure } \\
\text { correct schemas } \\
\text { are activated }\end{array}$ & $\begin{array}{l}\text { Options to post } \\
\text { comments on } \\
\text { role-model- } \\
\text { stories and films }\end{array}$ & $\begin{array}{l}\text { Population: Visiting teachers were } \\
\text { stimulated by an open question to post } \\
\text { comments. The open question structured } \\
\text { the discussion. } \\
\text { Context: The aim of the comments below } \\
\text { the role-model-stories and the film was } \\
\text { to stimulate a discussion between visiting } \\
\text { teachers about the topic discussed in the } \\
\text { story or film. It also served as a platform for } \\
\text { discussing tips on how to deal with that } \\
\text { specific difficulty. } \\
\text { Parameters: The placed reactions were } \\
\text { visible for all visiting teachers. A YouTube- } \\
\text { like structure was used for optimal usability. }\end{array}$ \\
\hline $\begin{array}{l}\text { Skills / Self- } \\
\text { efficacy }\end{array}$ & $\begin{array}{l}\text { Behavioral } \\
\text { Journalism }\end{array}$ & $\begin{array}{l}\text { Credible message, } \\
\text { model gives } \\
\text { reasons for } \\
\text { adopting new } \\
\text { behavior and } \\
\text { states perceived } \\
\text { reinforcing } \\
\text { outcomes received }\end{array}$ & $\begin{array}{l}\text { Rotating photo's, } \\
\text { role-model } \\
\text { stories \& films }\end{array}$ & $\begin{array}{l}\text { Population: Interviews with teachers were } \\
\text { used in several aspects of the website to } \\
\text { realize a platform by and for teachers. } \\
\text { Context: Photo's and interviews were used } \\
\text { to compose role-model-stories, films \& } \\
\text { photo's. These stories and films were based } \\
\text { on a structure in which first the problem is } \\
\text { presented as well as the experience and the } \\
\text { relevance of this problem followed by the } \\
\text { search for the most effective solution with a } \\
\text { description of failures and success factors. } \\
\text { Parameters: The interviewed teachers were } \\
\text { selected to present a diverse selection in } \\
\text { teaching experience, in geographic location } \\
\text { and personal characteristics and were } \\
\text { coping models, instead of mastery models, } \\
\text { to increase the identification. }\end{array}$ \\
\hline
\end{tabular}


Table 3. (continued)

\begin{tabular}{|c|c|c|c|c|}
\hline Determinants & Methods & Parameters & Applications & $\begin{array}{l}\text { How population, context and parameters } \\
\text { were taken into account }\end{array}$ \\
\hline $\begin{array}{l}\text { Skills / Self- } \\
\text { efficacy }\end{array}$ & Modeling & $\begin{array}{l}\text { Attention, } \\
\text { remembrance, } \\
\text { self-efficacy } \\
\text { and skills, } \\
\text { reinforcement } \\
\text { of the model, } \\
\text { identification } \\
\text { with model, } \\
\text { coping instead of } \\
\text { mastery model, } \\
\text { demonstrate } \\
\text { relevant skills }\end{array}$ & $\begin{array}{l}\text { Rotating photo's, } \\
\text { role-model } \\
\text { stories \& films }\end{array}$ & $\begin{array}{l}\text { Population: To create a platform for and by } \\
\text { teachers, teachers were interviewed which } \\
\text { formed the content for role-model-stories } \\
\text { and films. Photos of teachers were taken to } \\
\text { increase reliability and credibility as well as } \\
\text { to lure teachers to the website. } \\
\text { Context: The interviews were used to fill in } \\
\text { the main content of the website. } \\
\text { Parameters: Interviewed teachers were } \\
\text { selected on personal characteristics, on } \\
\text { geographic location and on experience } \\
\text { to create a database of diverse teachers } \\
\text { that the target group could identify with. } \\
\text { The interviewed teachers were all coping } \\
\text { models. }\end{array}$ \\
\hline \multirow[t]{2}{*}{ Attitude } & $\begin{array}{l}\text { Self- } \\
\text { reevaluation }\end{array}$ & $\begin{array}{l}\text { Feedback \& } \\
\text { confrontation; } \\
\text { however, raising } \\
\text { awareness must } \\
\text { be followed } \\
\text { by increase in } \\
\text { problem solving } \\
\text { ability and self- } \\
\text { efficacy }\end{array}$ & $\begin{array}{l}\text { Self-reflection } \\
\text { tool }\end{array}$ & $\begin{array}{l}\text { Population: Teachers expressed a } \\
\text { minimal need for coaching but teachers' } \\
\text { implementation behavior was characterized } \\
\text { by inconsistently selecting parts of the } \\
\text { program and not delivering all lessons. } \\
\text { To bring about behavioral change first a } \\
\text { self-reflection intervention is necessary } \\
\text { to create professional awareness as the } \\
\text { first step of improving implementation } \\
\text { behavior. } \\
\text { Context: In this self-reflection tool teachers } \\
\text { could score different aspects of their own } \\
\text { professional behavior in dealing with } \\
\text { difficulties on a Likert-scale. } \\
\text { Parameters: After completion of the } \\
\text { self-reflection tool an overview of gaps } \\
\text { in learning were revealed. The teachers } \\
\text { were directly referred to the most personal } \\
\text { relevant difficulties. }\end{array}$ \\
\hline & $\begin{array}{l}\text { Scenario- } \\
\text { based- } \\
\text { information }\end{array}$ & $\begin{array}{l}\text { Plausible scenario } \\
\text { with cause and } \\
\text { outcome, imagery }\end{array}$ & $\begin{array}{l}\text { Role-model- } \\
\text { stories \& films }\end{array}$ & $\begin{array}{l}\text { Population: Teachers were interviewed } \\
\text { to collect data and to form the content } \\
\text { for the scenario's. Teachers were coping } \\
\text { models who were also experiencing the } \\
\text { same problems as the target group but also } \\
\text { found a solution. } \\
\text { Context: In the films and stories interviewed } \\
\text { teachers were especially asked to describe } \\
\text { scenarios to make the learning process } \\
\text { applicable in daily practice and for the } \\
\text { individual situation of a teacher. } \\
\text { Parameters: The scenarios were described } \\
\text { according to a structure in which the } \\
\text { (personal) relevance and description of the } \\
\text { problem was made followed by a search for } \\
\text { the most effective solution. }\end{array}$ \\
\hline
\end{tabular}

Modeling

(see above)

Bartholemew et al., 2011 


\section{IM step 4: Program development}

In this step, the intervention program is designed and materials are developed. The intervention, called Lesgevenindeliefde.nl (Teaching Love), was designed as a coaching website for teachers.

Although teachers expressed a minimal need for coaching during the interviews in the needs assessment, the program developers nonetheless saw the need to develop Lesgevenindeliefde.nl for the following reasons: (1) Quality of implementation by teachers is suboptimal, despite their conviction about their own teaching method. To change this conviction and improve teacher implementation behavior, self-reflection and professional development are necessary. Teachers do not usually reflect on their own implementation behavior. Self-reflection, however, could lead to a critical evaluation and subsequently to improvement of their implementation behavior, which ultimately contributes to an increased effectiveness of an intervention. Coaching can only start when teachers develop an awareness of the need and a desire to improve their performance or change the way they have been performing at work (Parsloe \& Leedham, 2009). Confronting teachers with potential difficulties they could encounter might help them to reflect on their behavior. (2) Teachers could use support in adequately dealing with the difficult situations encountered when delivering SRH lessons, as mentioned in the needs assessment. This could lead to improved program implementation. (3) The development of a website is an efficient, low-threshold way of reaching a mass of teachers. It partly replaces and supports the implementation promoting tasks of the MHS, which now lacks the capacity and didactic expertise for training teachers. The choice of internet as a channel of the intervention was pre-determined by the program financers.

To ensure that the intervention was appealing and trustworthy to teachers, the coaching website was based on the concept 'by and for teachers', with role model stories, photos, and videos as the main products of this concept. This concept was chosen because teachers stated that if they did seek support during the delivery of SRH lessons, they preferred to consult fellow teachers or considered other teachers as reliable sources of information and for seeking advice. A large study in the Netherlands found that teachers in secondary schools either consult colleagues in their school for information or use the internet to find information, to prepare their lessons, send e-mails to students or give homework assignments (Zwaneveld et al., 2009). Studies in the U.K. pointed clearly to the value of teachers learning with and from each other when it comes to professional development (Cordingley, Bell, Rundell, Evans, \& Curtis, 2003; Cordingley, Bell, Thomason, \& Firth, 2005). 
Certain characteristics of the website such as its accessibility, usability, flexibility, and tailorability to needs of teachers potentially limited barriers to visiting the website. Teachers could flexibly access all the information on the website that was personally relevant rather than being forced through a fixed coaching program. This catered to their lack of time and diverse needs for support. Accessibility to the website was simplified by placing the link on the LLL website under the 'teacher' button. Teachers were encouraged to come back to the website by constantly placing new updates there and by integrating teacher materials in the website.

The Web-based intervention was developed with the underlying idea of an unobtrusive coaching technique whereby teachers' actual needs were addressed and their perceived need of coaching was changed. With this technique, we attempted to trigger teachers to become aware of their need for coaching, without awakening resistance, by exposing them to difficulties experienced by other teachers they identified with. In order to meet teachers', need for student materials, teaching materials were provided via the website. This student material can function as the first trigger for teachers to visit the website but was strategically placed at the bottom of the navigation system to ensure that teachers were first exposed to the most common difficulties encountered during implementation. It's a necessity that teachers effectively cope with difficulties to prevent these from becoming a barrier to optimal delivery of the program. Subsequently, elimination of these barriers is followed by practical support in delivering the lessons to ultimately accomplish complete and fidel delivery of Long Live Love.

Furthermore, teachers were lured into the deeper structure of the website by presenting clickable rotating quotes with photos of teachers they could identify with. This is in line with the concept 'by and for' teachers derived for the method of peer coaching. Peer coaching suggests that the professional development of teachers can be improved through experimentation, observation, reflection, the exchange of professional ideas, and shared problem-solving (Zwart, Wubbels, Bergen, \& Bolhuis, 2007). The information on the website was given by teachers instead of experts as they are coping models, not mastery models, which is important for the acceptance of the message (Bartholemew et al., 2011).

The homepage of the coaching website includes a left-menu structure and rotating photos of teachers with SRH-teaching related quotes. These photos of teachers with short rotating quotes, placed in the center of the homepage, were meant to increase teachers' awareness of the most common difficulties and to trigger their perceived need to be coached. These quotes also served as cues for teachers to browse further through the website and as an entrance to the related role model stories. Furthermore, 
on the homepage, general information about the website could be found as well as the possibility to ask for support via email, up-to date information about SRH, a search function and FAQ (see Figure 2). Additional subpages could be reached by clicking on any of the features presented on the homepage.

To access the website, teachers had to sign up with a self-created user name and password. The sign-up was included to protect the privacy of teachers and to provide a protected internet environment where teachers could safely exchange their ideas. The website also needed to be secured to prevent students from accessing it. For the program developers, these account details provided demographic information about the users and the use of the website.

Access to the main content of the website was predominantly navigated through the left-menu structure. This structure contains three categories: (1) A self-reflection tool to trigger teachers to reflect critically on their implementation behavior, (2) the eight main difficulties that teachers may face when implementing SRH lessons, each with their own underlying sub-homepage, and (3) student materials and practical instructions teachers need to implement LLL completely and with fidelity. See Figure 2 for an impression of the homepage.

The left-menu structure was chosen for usability purposes, mainly because it presented the website content and structure clearly. This made the website accessible and easy to use. Current practices strongly recommend placing the main navigation menu on the left-hand side of the page (Nielsen, 1999). The choice of this navigation system addressed teacher's limitations of time and skills in internet use, and the general preference of website visitors to be able to have a clear navigation on the homepage (Crutzen, Cyr, \& de Vries, 2012).

The first part of the left menu structure consisted of a self-reflection tool. This was developed to enable teachers to reflect critically on their own implementation behavior and to create awareness of their need for coaching concerning the most common difficulties in providing SRH. The self-reflection tool was developed in the form of an interactive questionnaire whereby each statement related to teaching SRH was rated on a Likert scale of 1 (totally disagree) to 7 (totally agree). For example, on the difficulty of teaching SRH without shame, the following statement had to be rated from 1 to $7:$ :I can deal with feelings of shame in such a way that they do not limit my lesson' (see Figure 3). With the results of the reflection tool, teachers were referred to personally relevant pages on the website to enable them to improve these specific behaviors. 


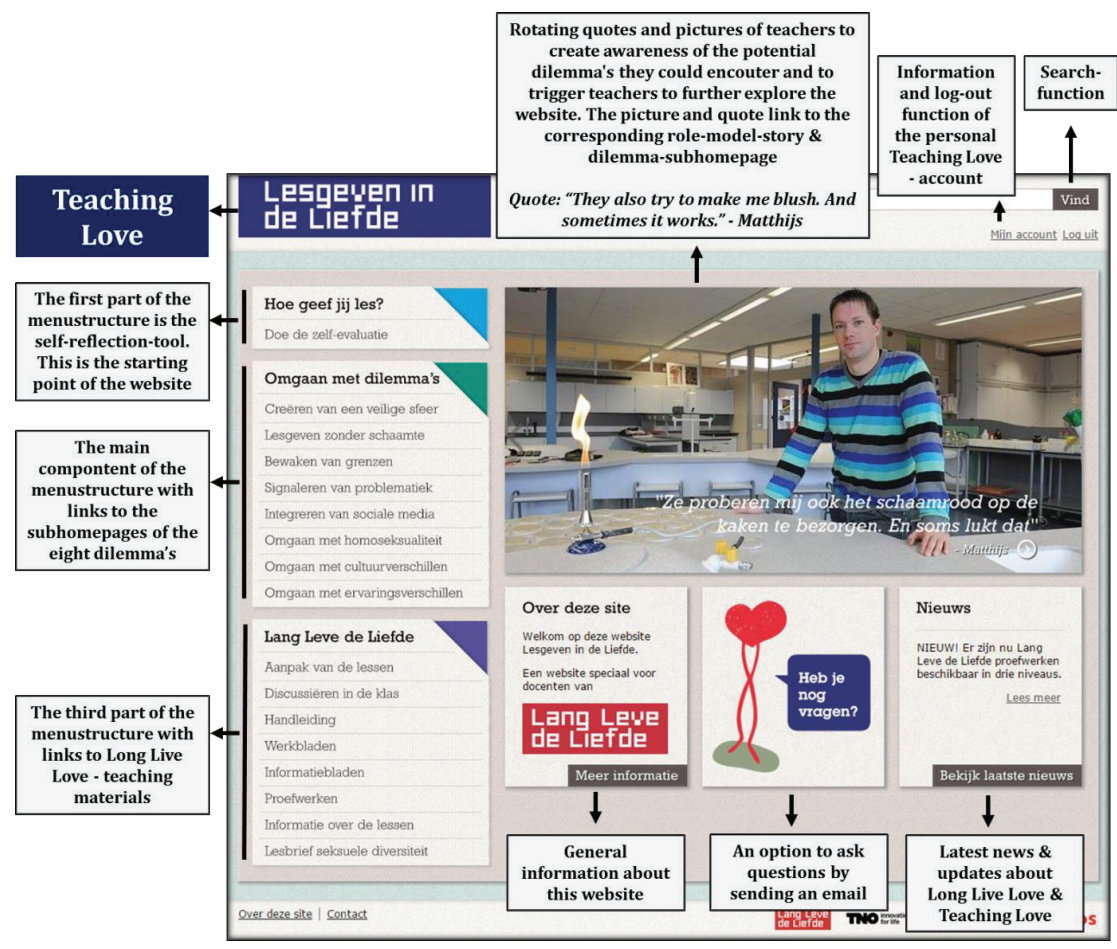

Figure 2. Homepage of Lesgevenindeliefde.nl.

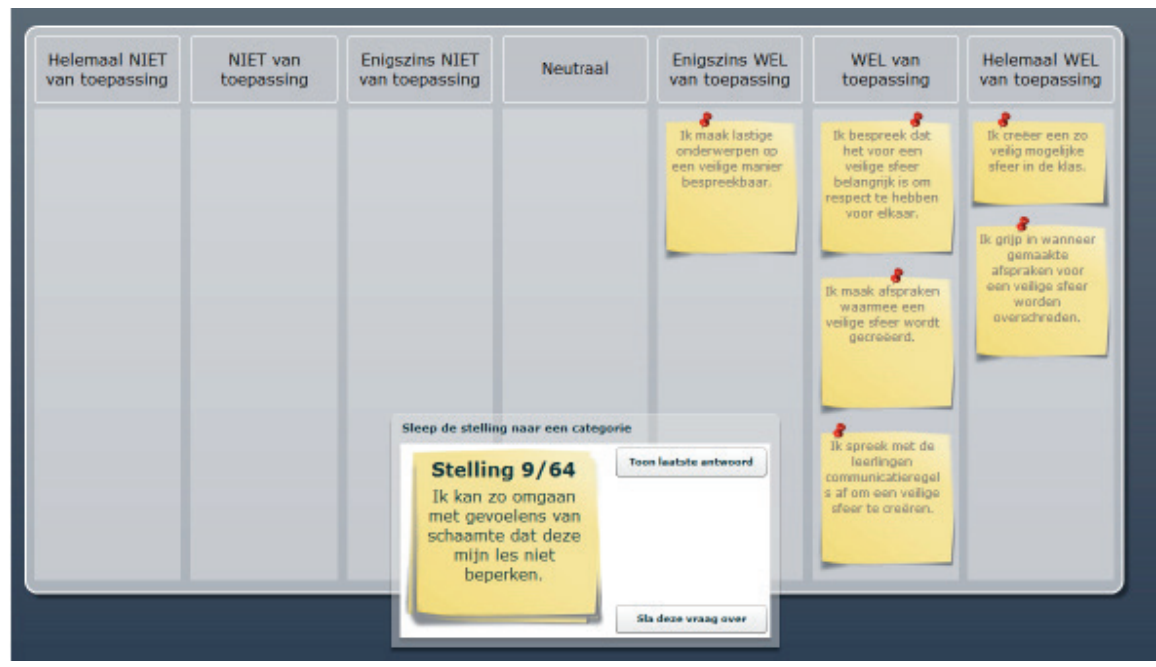

Figure 3. The self-reflection tool. 
The central component of the left menu structure focused on the eight main difficulties encountered during implementation by presenting them via eight separate buttons, each with its own sub-homepage. The difficulties were: (1) creating a safe and secure atmosphere for the provision of SRH lessons, (2) teaching SRH without shame influencing the quality of the lessons, (3) protecting boundaries in sharing personal information and questions between teachers and students and among students themselves, (4) identifying personal problems of students, (5) integrating social media in SRH lessons, (6) integrating homosexuality as self-evident during the lessons of LLL and dealing with negative reactions to homosexuality and anticipating on (7) differences in culture, and (8) different levels of sexual experience in the classroom.

By clicking on one of these menu items, the visitor entered a sub-homepage with background information on the specific difficulty, videos, role model stories with rotating quotes and suggestions to adequately deal with that specific difficulty. The rotating quotes served the same function as the quotes on the homepage, namely to make them aware of the personal relevance of the difficulty, awaken their need to adequately deal with that difficulty and trigger them to access further content. Each role model story and video had the possibility of posting a comment, similar to existing commentary structures, such as YouTube (See Figure 4).

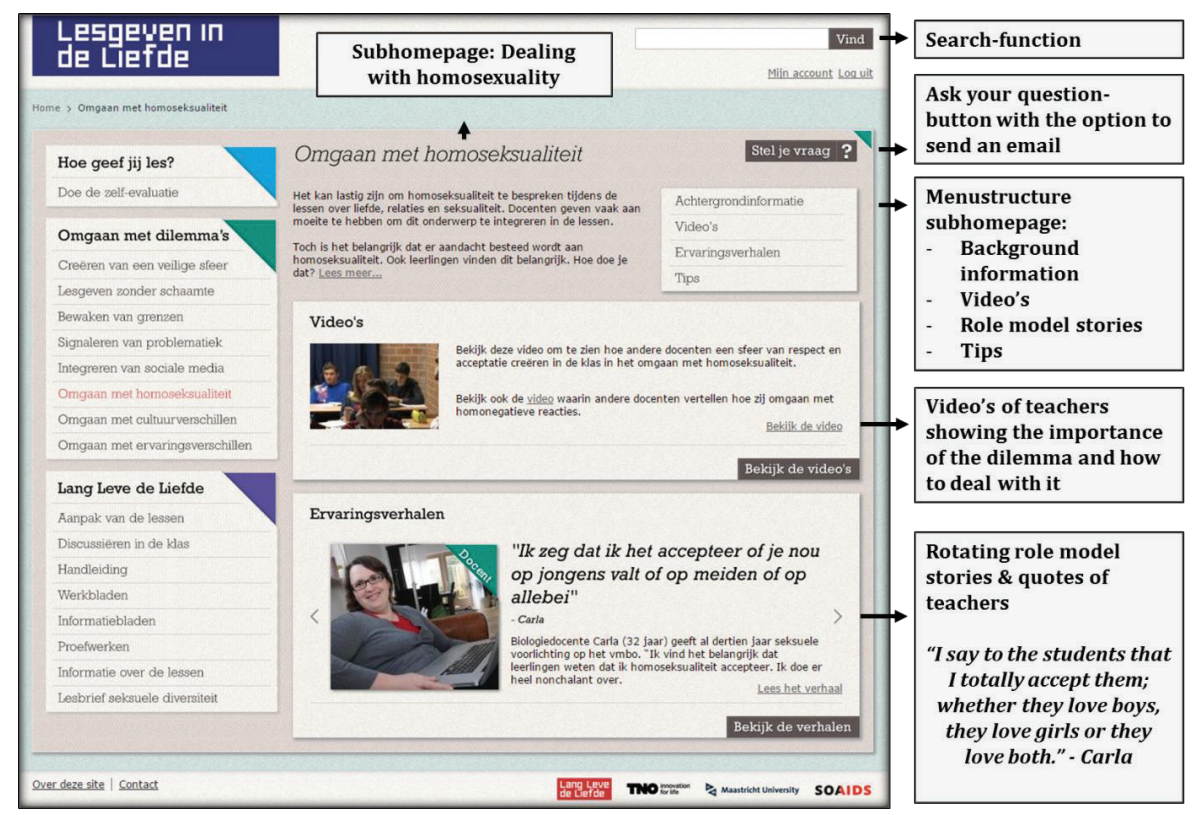

Figure 4. Sub-homepage: Dealing with homosexuality. 
The content of the website was mainly obtained by interviewing teachers for role model stories and videos. It was important that the videos and role-model stories were realistic; teachers had to be able to identify with them, the content had to be recognizable and a clear solution on how to cope with the difficulty had to be provided. Each video and role model story was based on the structure of first introducing the teacher to the difficulty and then providing a solution. First knowledge, then awareness, attitude, and then skills were addressed in these video's and role model stories. The role model first introduced and acknowledged the difficulty (knowledge \& awareness), then expressed the importance of dealing adequately with the difficulty (attitude) and finally described how (s)he effectively coped with the difficulty in such a way that it did not influence the implementation of LLL (see example of role model stories in Figure 5). The role model stories and videos were supplemented by general suggestions including didactic and pedagogic information, and background information concerning the difficulty. This information was collected from websites and articles as well as from own research.

\begin{tabular}{|c|c|}
\hline Create a safe and secure atmosphere & Teaching without shame \\
\hline $\begin{array}{l}\text { 'Ze mogen geen namen noemen en } \\
\text { elkaar niet uitlachen' }\end{array}$ & $\begin{array}{l}\text { Ik ben soms nog steeds een beetje } \\
\text { zenuwachtig als ik aan dit }\end{array}$ \\
\hline 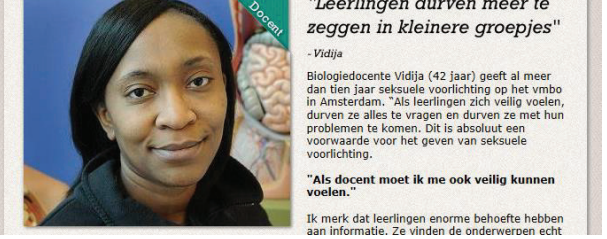 & 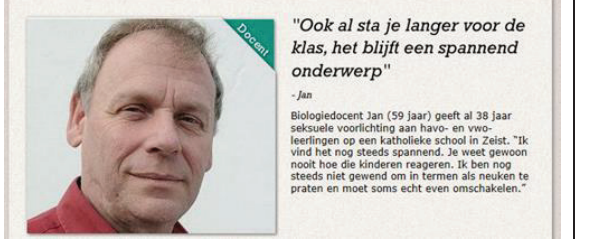 \\
\hline "Students dare to say more in smaller groups" & $\begin{array}{l}\text { "Even when you are experienced in teaching sex } \\
\text { education, it remains nerve wrecking." }\end{array}$ \\
\hline $\begin{array}{l}\text { Vidija - Biology teacher, } 42 \text { years old: “When students } \\
\text { feel safe, they dare to ask anything and come forth } \\
\text { with their problems. This is an absolute condition when } \\
\text { providing sex education. I notice that students have } \\
\text { many questions. It's important to give them room for } \\
\text { this in these lessons. However, as a teacher I should } \\
\text { also feel safe. That's why I make an agreement with the } \\
\text { students to respect them and expect them to respect } \\
\text { me in return. We make straightforward agreements: } \\
\text { listen to one another, don't laugh at each other and don't } \\
\text { mention names when telling stories. If they start saying } \\
\text { 'she did this and that' I cut them off immediately. I also } \\
\text { get students to work in smaller groups when it comes to } \\
\text { certain subjects like 'loverboys'. Students then dare to say } \\
\text { more and are more open than when you discuss it with } \\
\text { the entire classroom. }\end{array}$ & $\begin{array}{l}\text { Jan - Biology teacher, } 59 \text { years old: "I still find it thrilling. } \\
\text { You just never know how the kids will react. I'm still } \\
\text { not used to using words like'screwing' and really have } \\
\text { to switch my mind-set. To reduce the tension, I allow } \\
\text { the students sometimes to write all the words on the } \\
\text { board that have to do with sex. It's important that they } \\
\text { can laugh. It also helps me to prepare my lessons well } \\
\text { in advance so that I know what my students are going } \\
\text { through. What issues are they going through? What } \\
\text { questions can I expect? I am still a bit nervous when I start } \\
\text { this topic but I think that's natural and human. }\end{array}$ \\
\hline
\end{tabular}

Figure 5. Examples of role model stories. 
The last part of the left-menu structure was specifically aimed at effective practical delivery of the Long Live Love program. Optimal delivery was achieved by informing teachers how to best provide LLL lessons and how to handle discussions in the classroom by means of role model stories and videos. Teachers could also download teaching materials such as the teacher manual, work- and information sheets for students and general information about the LLL curriculum. These materials were strategically included in the website to lure teachers to the website and meet the need they expressed in the needs assessment for such teaching tools.

\section{IM Step 5: Implementation}

The coaching website (Lesgevenindeliefde.nl) had to be used by teachers in order to have an impact on program effectiveness and ultimately on student outcomes. In this step, an implementation plan was made to ensure that teachers were aware of the existence of the website and made use of it. Despite being designed to support teachers in their implementation of LLL, the website itself also needed to be effectively implemented.

The implementers of the coaching website are the program developers, STI Aids Netherlands, who maintained, monitored and updated the website and made it available online and easily accessible. Teachers were the end users of the website. The implementers developed implementation tools, according to the Intervention Mapping protocol, to ensure that teachers were exposed to the website and to increase awareness and use of the website. An information leaflet including information about Long Live Love and the website was created. Additionally, a trailer of the website was developed, explaining the aim of the website and showing the content and use of it (see Multimedia Appendix 1). This trailer and further information about the website were also integrated into the training provided to teachers by the MHS. In this training, teachers were informed by the MHS about LLL as well as the existence, advantages and use of the coaching website, thereby stimulating teachers to use it when implementing LLL.

To further ensure teachers' awareness of the website and ability to make use of it in an efficient manner, a link to the website was integrated into the teacher manual. In the manual, references were made to the website in each lesson where relevant or wherever a specific difficulty was expected to arise in that lesson. The aim and functionalities of the website were also described in the teacher manual. 


\section{IM Step 6: Evaluation}

In this step of Intervention Mapping (IM), an evaluation plan and the corresponding evaluation measures were identified and developed. An effect- and process evaluation for the pilot implementation of the coaching website, Lesgevenindeliefde.nl, was performed. This occurred simultaneously with the pilot implementation of the schoolbased LLL intervention for students (Hofstetter et al., 2014). Formulated outcomes of steps 2 and 5 of IM, namely the change objectives and the implementation goals, were used in creating the evaluation plan. The aim is to find out how and to what extent teachers made use of the website, how they appreciated it, what effects it had on their (complete and fidel) implementation of LLL and what factors affected teacher's use of the website. This was investigated using a randomised controlled trial design. Qualitative and quantitative data was collected. Results of this study will be described elsewhere.

\section{DISCUSSION}

In this paper, the Intervention Mapping (IM) protocol was applied for the development of a theory-and evidence-based Web-based coaching intervention, Lesgevenindeliefde. $\mathrm{nl}$ (Teaching Love), aimed at supporting teachers in their implementation of the sex education program, Long Live Love (LLL). The IM protocol is perceived as a useful tool for guiding the development of this intervention and making it one which is compatible with the needs and preferences of teachers. With the IM protocol, careful decisions were made using a cumulative and iterative process, resulting in this Web-based implementation support intervention by and for teachers. Although IM was useful in designing this intervention, it is also a time-intensive method which was exacerbated by limited available resources in the project. Predetermined requirements of the program financers, such as budget and time, and available capacity and time of the MHS restricted the options for the type of intervention such as a digital versus a personal form of coaching. The method of IM used to develop the e-coaching intervention can be applied in other school settings or extrapolated to other areas of health promotion (Bartholemew et al., 2011).

The first step in the IM process revealed an interesting and challenging discrepancy between teachers' actual implementation behavior and their perception of their behavior. Several studies revealed that there is an implementation problem among teachers but teachers themselves do not perceive this sub-optimal implementation behavior as a problem (Bessems et al., 2014; Peters et al., 2009; Dusenbury et al., 2003; Dusenbury, Brannigan, Hansen, Walsh, \& Falco, 2005; Martens, Van Assema, Paulussen, 
Schaalma, \& Brug, 2006). In the case of LLL, teachers did not deliver lessons completely and according to the goals of program designers but teachers did not perceive this behavior as problematic or as a necessity to effectively provide the lessons. This behaviour, however, could possibly result in decreased program effectiveness. Teachers did, however, acknowledge some difficulties encountered when providing sex education, who according to more experienced teachers, were predominantly faced by less experienced colleagues. These difficulties could affect the fidelity and completeness if they are not adequately dealt with.

Teachers remain a difficult target group for bringing about behavioral change and made it challenging to develop a coaching intervention. Although the intervention was necessary, teachers expressed resistance and reluctance, and experienced a limited intrinsic motivation to be coached, thereby rendering directive coaching as a noneffective method for behavioral change of this target group. Directive coaching has been found to be an effective method to expose teachers to the intervention content but eagerness is a necessity prior to entering and being involved in this directive coaching process (Crutzen et al., 2010). Lack of intrinsic motivation namely forms a barrier to coaching (Fielden, 2005). Without a need and desire to be coached it is almost impossible to change behavior. Coaching can only start when teachers develop an awareness of the need and desire to improve their performance or change the way they have been doing things at work (Parsloe \& Leedham, 2009). This resulted in selecting the technique of unobtrusive coaching to create awareness without creating resistance; a non-directive way to bring about behavioral change. To achieve behavioral change, self-reflection is an essential first step to create intrinsic motivation to be coached and to ultimately develop professionalism in SRH. Additionally, a concept of peer coaching was integrated, by and for teachers, to lower the resistance and to ultimately achieve behavioral change. Peer coaching suggests that the professional development of teachers can be improved through experimentation, observation, reflection, the exchange of professional ideas and shared problem solving (Zwart et al., 2007).

To lure teachers to the website, student materials were made available online. The lack of intrinsic motivation to visit the website triggered program developers to invest in additional implementation activities because a website alone would be insufficient to involve teachers who are not intrinsically motivated. Integrating the website in the teacher manual, incorporating information about the website in teacher training and developing a trailer to create awareness and enthusiasm amongst teachers for the website are examples of implementation activities. 
The increased use of digital technologies in the education system, such as Lesgevenindeliefde.nl, brings exciting opportunities for innovative ways of teaching and learning. New, online technologies do not only provide an anonymous communication space but also offer students and teachers easier, affordable, convenient and faster access to information, teaching and learning resources, peers, experts, and a wider community. Online technology is also a low-threshold and efficient way of reaching many teachers and providing support in, for example, the implementation of schoolbased programs. Exploring the educational potential of these digital technologies and supporting schools in making optimum use of them remains important (Ertmer, \& Ottenreit-Leftwich, 2010).

\section{CONCLUSIONS}

With the development of the e-coaching website, a unique contribution was made in the field of bringing about behavioral change amongst intermediaries, especially due to the elements of self-reflection and unobtrusive peer coaching. The use of Webbased coaching to improve implementation behavior of teachers could be generalized to different cultural contexts since it addresses the common challenges faced in the area of sexual health education in schools worldwide (Kirby, Laris, \& Rolleri, 2007). Our process of intervention development may be applied to get from problem to solution regarding diverse implementation problems in development of interventions for challenging target groups. Also, other health promoting professionals may benefit from our example of the ongoing process of balancing input of the target group with the wishes of the intervention developers to ultimately develop an effective intervention.

Lesgevenindeliefde.nl will be tried out in practice by means of a pilot implementation. During this pilot-implementation, the website will be evaluated on process and effect. Based on the experiences of teachers and outcomes of the studies, further enhancements of the website could be made. The introduction of an innovation, such as Lesgevenindeliefde.nl, could present certain challenges in the implementation phase. The process of accepting the innovation takes time, as described in Rogers' diffusion curve (Rogers, 2003). The innovation will most likely be adopted by innovators first, followed by the early majority and eventually the laggards. Pijpers, van Montfort, \& Heemstra, (2002), claim that Web-based innovations first need to be accepted in order to be used broadly and effectively. The systematic approach and customized concept of Lesgevenideliefde.nl can serve as a distinct example of how to bring about behavioral 
change in a target group of intermediaries who lack intrinsic motivation for and have resistance to coaching. This approach can be applied to other SRH programs in the school setting and to target intermediaries in health promotion.

\section{ABBREVIATIONS}

IM: Intervention Mapping

LLL: Long Live Love

MHS: Municipal Health Services

SRH: Sexual Reproductive Health 

CHAPTER 5

\section{Effect evaluation of a Web-based}

\section{coaching intervention to support} implementation of sex education among secondary school teachers: Randomized controlled trial

This chapter is resubmitted as:

Schutte, L., Mevissen, F.E.F., Meijer, S., Paulussen, T., van Empelen P. (2017). Effect evaluation of a Web-based coaching intervention to support implementation of sex education among secondary school teachers: Randomized controlled trial. Journal of Medical Internet Research. 


\section{A BSTRACT}

Background: The quality of implementation is important for the effectiveness of behavioral change interventions. Implementing such programs with completeness and fidelity is not an automatic process and may require additional support. In school settings, the support teachers receive during implementation is often limited and appears to fall short when attempting to preserve completeness and fidelity in program delivery. With the aim to improve completeness and fidelity of teachers' delivery of a sexual health promoting intervention ('Long Live Love') in secondary education, a webbased e-coach was developed ('lesgevenindeliefde.nl' - 'teachinglove.nl'). An effect evaluation was performed to evaluate the effectiveness of the e-coach, as part of a broader implementation strategy, in influencing teachers'implementation.

Objective: This paper reports on the effect evaluation to determine the effect of the web-based e-coach on (determinants of) teacher implementation of a school-based sexual education program called Long Live Love (LLL).

Methods: A cluster randomized controlled trial (e-coaching vs waiting list control) was conducted with a baseline assessment (T0) and follow up (T1) two weeks after completing the LLL program. A total of 43 schools with 83 teachers participated in the study. In the follow-up 38 schools participated, 23 in the e-coaching condition with 41 teachers, 15 in the control condition with 26 teachers. Multilevel regression analysis was used to evaluate the effect of the e-coaching website, on (determinants of) completeness and fidelity of LLL implementation.

Results: The e-coaching intervention was not found to have an effect on teachers' implementation behavior; teachers assigned to the experimental e-coaching website did not score higher on completeness ( $P=.60$; Regression weight $=-2.12$ (3.99); $95 \%$ C.I. $=-10.26,6.02 ;$ Cohen's $d=.17)$ or fidelity $(P=.67$; Regression weight $=0.09(0.21) ; 95 \%$ C.I. $=-0.33,0.51$; Cohen's $d=.14$ ) as compared to teachers in the control condition. When comparing the 30 teachers who made actual use of the e-coaching website with the 37 teachers who did not, no significant differences were found either $(P$ 's $\geq .54)$. There was also no effect of e-coaching on the determinants of teacher implementation behavior $\left(t^{\prime} s ; \leq 0.69 ; P^{\prime} s \geq .22 ; .33>\right.$ Cohen's $\left.d>.06\right)$.

Conclusion: E-coaching was not found to be effective in enhancing completeness and fidelity of LLL by teachers. The lack of effect might be attributed to the intervention content, the limited use, and/or the study design itself. The e-coaching intervention may not have adequately addressed fidelity and completeness to bring about behavioral 
change. Besides, the e-coaching intervention was not or insufficiently used by teachers, with a possible sample bias and 'ceiling effect'. This, however, does not imply that webbased coaching in itself is an ineffective strategy to promote fidelity and completeness of program implementation. A process evaluation is required to investigate teachers' appreciation, use and (motives for) use of the e-coach to further understand why e-coaching was not used and had no effect. 



\section{INTRODUCTION}

Correct implementation is important for the effectiveness of an intervention. An intervention that is implemented completely and according to its' guidelines is more likely to be successful in changing the target groups' determinants and behavior than programs that are not implemented fully (Durlak \& DuPre, 2008; Lendrum \& Humphrey, 2012; Little, Riggs, Shin, Tate, \& Pentz, 2015). In school-based sexual health promotion, teachers are the key players for the implementation of these programs. Their implementation is, however, often sub-optimal; Programs are not being implemented completely or with sufficient fidelity to produce measurable outcomes (Bessems et al., 2014; Gottfredson \& Gottfredson, 2002; Hoekstra et al., 2009; Peters et al., 2009; Schutte et al., 2014). There is a need for greater attention to the quality of implementation and its related determinants, namely teachers' beliefs about the innovation and characteristics of the innovation, organisational factors and characteristics of the implementationenhancing intervention (Fagan \& Mihalic, 2003; Paulussen et al., 1995; Schutte et al., 2014).

Teachers appear to be in need of support in every phase of the implementation process to enable them to put the innovation into practice (Durlak \& DuPre, 2008; Rohrbach et al., 2010; Bishop et al., 2006). Supporting teachers in the implementation phase has, however, been insufficiently considered, as most work has been invested to promote teachers' awareness and adoption of new interventions (Bessems et al., 2014; Domitrovich \& Greenberg, 2000; Fagan \& Mihalic, 2003; Durlak, 1998). This applies in particular to school-based sex education programs, which address the sensitive subject of sexuality. Providing support prior to implementation in the form of training often equips teachers with skills for correct implementation, but it is not enough (Dusenbury et al., 2003). It remains important to provide teachers with more personal assistance and ongoing support and consultation during the process of putting an innovation into practice (Schutte et al., 2014; Kramer et al., 2000; Rohrbach et al., 1993; Durlak, 1998). Currently, this support is limited to providing practical support in the form of teacher manuals with practical information on the content of the lessons and on how to deliver such lessons. However, more in-depth coaching focusing on determinants of implementation such as self-efficacy and social support to enhance completeness and fidelity is lacking (Bartholomew et al., 2016; Farmer-Dougan et al., 1999; Hansen et al., 2009; Jones et al., 1997; Kam et al., 2003; Mihalic et al., 2008; Mortenson, \& Witt, 1998; Noell et al., 1997; Rohrbach et al., 2010; Witt et al., 1997). 
To stimulate the correct implementation, with completeness and fidelity, of the (revised) school-based sex education program'Long Live Love'(LLL) (Schaalma et al., 1996; Schutte et al., 2014), an e-coaching intervention ('lesgeveinindeliefde.nl' - 'teachinglove.nl') was systematically developed (Schutte, van den Borne, Kok, Meijer, \& Mevissen, 2016). The e-coach aimed to improve teachers' implementation behavior through self-reflection and skills development. Through e-coaching, we aimed at making teachers aware of the importance of completeness and fidelity in relation to program effectiveness and increase their awareness regarding their own (suboptimal) implementation behavior. Also, the e-coach provided tools to help teachers improve their implementation by giving support on how to deal adequately with potentially difficult classroom situations they could encounter when providing Sexual and Reproductive Health (SRH) lessons, such as creating a safe atmosphere in the classroom for students to openly discuss relationships and sexuality, handling personal questions addressed to teachers by students and intervening on homonegative remarks or behavior. The content of the e-coach was guided by theories on implementation behavior (Paulussen et al., 1995; Durlak \& DuPre, 2008), and based on a needs assessment among the target group (Schutte et al., 2016). Intervention objectives were psychosocial determinants, such as awareness, teachers' personal benefit, social support, (anticipated) student responses, and self-efficacy. The E-coach could be used by teachers prior to and during deliverance of the LLL program. For a more detailed description of the e-coach see Schutte et al., 2016 (Schutte et al., 2016).

The E-coach was part of a broader implementation strategy aimed at promoting each phase of the LLL implementation process. The Municipal Health Services (MHS) were involved in the implementation strategy. This strategy included instruction protocols used by the MHS to promote adoption and continuation of LLL by teachers in schools, a teacher training delivered by the MHS, and a teacher manual to enhance and facilitate implementation. The MHS training was aimed at introducing the revised LLL program to teachers and motivating them to use the program and use it as intended by enhancing teacher's knowledge, attitudes and skills. The training was provided prior to implementation of LLL and was followed by e-coaching. An effect and process evaluation for the pilot implementation of the coaching website was conducted. This occurred simultaneously with the pilot implementation of the revised school-based LLL intervention for students (Hofstetter et al., 2014). This paper focuses on the effect of e-coaching on (determinants of) teachers' implementation behavior. The process evaluation is described elsewhere (personal communication by Schutte et al., 2017). 


\section{METHODS}

\section{Design}

A clustered randomized controlled trial (e-coaching vs waiting-list control) was conducted, with a baseline assessment (T0) and follow up (T1) two weeks after completing the LLL program. Teachers were not informed about the existence of these two groups.

\section{Recruitment \& Procedure}

From all the secondary schools in the Netherlands ( $N=610)$, a sample of $\mathrm{N}=115(19 \%)$ schools were randomly selected, after stratification according to region and education level (preparatory applied education, higher general continued education and preparatory scholarly education). Teachers within these schools were invited, by e-mail and telephone, to use the revised LLL program and to participate in a survey study on their experience and implementation of Sexual and Reproductive Health education (SRH) and LLL. Only teachers who taught SRH were contacted. The schools with teachers who accepted the invitation $(\mathrm{N}=45)$ were randomly assigned to either the control or the intervention (e-coach) group.

Teachers in the intervention and control group who consented to participation first received the baseline survey (T0) by post. The T0 survey focused on (determinants) of SRH and LLL implementation and took approximately 30 minutes to fill out. Teachers had two weeks to complete and return the survey. Non-responders got a reminder by e-mail and eventually by telephone three days after the deadline and were given another two weeks to return the survey.

At the same time, teachers in both groups were offered a training from the MHS in their region prior to implementing the revised LLL program. Separate trainings were delivered to teachers in the e-coach intervention group vs. the control group with teachers in the intervention group receiving additional information during the training about the e-coaching website and being stimulated to use it during the implementation of LLL. In the end, $58.2 \%$ ( $N=39 ; n=24$ from the intervention group; $n=15$ control group) of the participating teachers in the survey received a training from 14 different MHS's. The remaining teachers did not receive training, either because they refused the training as they felt there was no need or because the MHS in their region was not offering the training. 
Teachers in both groups then received the LLL program (a package including a student magazine, a student DVD, and a teacher manual) by post mail, which they could implement within (approximately) two months following the baseline measurement for teachers (T0). Additionally, teachers in the intervention group were given access to the e-coaching website with a personal user name and password, and an edition of the LLL teacher manual which contained references to the website. The teachers in the control group were not exposed to or informed about the website, until after the end of the e-coach evaluation. They received the regular LLL teacher manual without any references to the e-coach. Halfway during the pilot implementation, an e-mail reminded teachers in the intervention group to use the e-coaching website. One week before expected completion of the LLL program, all teachers were reminded by e-mail and telephone about the upcoming post-test survey (T1). Within two weeks after completing the implementation of the LLL program, the T1 survey was sent to all teachers. Reminders were sent by e-mail and eventually by telephone to non-responders. All procedures in the study were approved by the authorized Ethical Review Committee of Psychology \& Neuroscience (ERCPN) at Maastricht University. Registration of this trial was not required.

\section{Measurements}

The survey used for the effect evaluation focused on determinants targeted by the e-coach and was based on the theoretical framework explaining teachers' adoption and implementation of SRH developed by Paulussen et al. (1994), which is a combination of the Theory of Planned Behavior (TPB) (Ajzen, 2011), Social Cognitive Theory (SCT) (Bandura, 1986) and Diffusion of Innovations theory (Dol) (Rogers, 2003). Further description of and foundation for this framework can be found in Schutte et al., (2014). At baseline (TO) we measured background characteristics of the teachers, including (SRH/LLL) teaching experience and their LLL-curriculum related beliefs, and student response. At post-test (T1) we measured the same determinants but also included measures on completeness and fidelity of LLL implementation. In addition, subjective evaluations of the e-coach and the MHS training were included (this will be further discussed in the process evaluation (personal communication by Schutte et al., 2017).

Demographic variables (T0) included gender, age, teaching subject, educational level of students, years of teaching experience, years of teaching $\mathrm{SRH}$, perceived expertise in teaching $\mathrm{SRH}$, perceived need for support in providing $\mathrm{SRH}$, attitude towards teaching $\mathrm{SRH}$, attitude towards reflecting on own SRH teaching methods, past experience with previous versions of LLL and sexual morality. 
Outcome measures; Table 1 provides an overview of all measures. For measuring curriculum-related beliefs (TO \& T1): Teacher benefits, subjective norms social support and self-efficacy were assessed together with (anticipated) student responses.

Teacher's implementation behavior (T1) was measured based on rates of completeness and fidelity of LLL implementation. Completeness was expressed by the proportion of the 19 core learning activities of the LLL program being implemented ( $\Sigma$ implemented activities/19*100).

Fidelity was measured as the extent to which the LLL program was implemented according to the guidelines as prescribed in the teacher manual, with scores ranging from 1 ("I reviewed the program and only delivered a few lesson suggestions according to the teacher manual), to 5 ("I delivered all lesson suggestions for the LLL program exactly according to the teacher manual") (Hall, Dirksen, \& George, 2006). All measures, including number of items, response scales, reliability and exemplary items are presented in Table 1.

\section{Analyses}

Data were analysed using SPSS24. Given the nested structure of the design and the data (partly repeated measurements, nested within teachers, nested within schools) multilevel regression analyses were used to evaluate the effects of e-coaching on teachers' (determinants of) implementation of LLL. Two levels were defined in the multilevel analysis: (1) school, (2) teacher.

The model included the predictors group ( 1 for intervention group (e-coach), 0 for control group) for the outcomes of implementation behavior (completeness and fidelity) and group, time of measurement (baseline and post-test) and the interaction time $\mathrm{x}$ group for the determinants. 
Table 1. Measures, number of items, reliability, example items and answer scale.

\begin{tabular}{|c|c|c|c|}
\hline & Items & a & Exemplary items (response scales) \\
\hline \multicolumn{4}{|l|}{ Background variables } \\
\hline Gender & 1 & & What is your gender? $(0=$ female, $1=$ male $)$ \\
\hline Age & 1 & & What is your age? \\
\hline Teaching subject & 1 & & $\begin{array}{l}\text { What subject do you teach? } \\
(1=\text { biology, } 2=\text { health care, } 3=\text { citizenship, } 4=\text { other })\end{array}$ \\
\hline Years of teaching experience & 1 & & How many years have you been working in education? \\
\hline Years teaching SRH & 1 & & How many years have you been teaching SRH? \\
\hline Perceived expertise teaching SRH & 1 & & $\begin{array}{l}\text { How experienced are you in teaching SRH? } \\
\text { ( } 1=\text { very inexperienced, } 7=\text { very experienced })\end{array}$ \\
\hline $\begin{array}{l}\text { Perceived need for support in } \\
\text { providing SRH }\end{array}$ & 1 & & $\begin{array}{l}\text { Do you need support in providing SRH lessons? } \\
(1=\text { no, certainly not, } 5=\text { yes, certainly })\end{array}$ \\
\hline Attitude towards teaching SRH & 6 & .82 & $\begin{array}{l}\text { Indicate what you think about teaching SRH: Teaching SRH is } \\
\text { [important, necessary, fun, difficult, comfortable, competent] } \\
\text { ( } 1=\text { not at all, } 7=\text { yes, totally) }\end{array}$ \\
\hline $\begin{array}{l}\text { Attitude towards reflecting on own } \\
\text { SRH teaching methods }\end{array}$ & 1 & & $\begin{array}{l}\text { Indicate what you think about reflecting on your own SRH } \\
\text { teaching methods: [important, useful, good] } \\
\text { ( } 1=\text { not at all, } 7=\text { yes, totally) }\end{array}$ \\
\hline Use of previous LLL & 1 & & $\begin{array}{l}\text { Have you used the previous LLL in the past for SRH lessons? } \\
(0=\text { no, } 1=\text { yes })\end{array}$ \\
\hline Years teaching LLL & 1 & & For how many years have you been using Long Live Love? \\
\hline Sexual morality & 5 & .62 & $\begin{array}{l}\text { Young people who have just met should not have sex } \\
\text { ( } 1 \text { = strongly disagree, } 5=\text { strongly agree })\end{array}$ \\
\hline \multicolumn{4}{|l|}{ Curriculum related beliefs } \\
\hline Teacher benefits & 6 & .72 & $\begin{array}{l}\text { I gained insight in the sexuality experience of youngsters. } \\
(1=\text { strongly disagree, } 5=\text { strongly agree })\end{array}$ \\
\hline Subjective norms & 6 & .81 & $\begin{array}{l}\text { Do you think that the following people appreciate you using } \\
\text { Long Live Love to provide sexual education? [principal, governing } \\
\text { body, external consultants/health education experts, students, } \\
\text { colleagues teaching the same and colleagues teaching a different } \\
\text { subject, parents] } \\
\text { (1 = no, certainly not, } 5 \text { = yes, certainly) }\end{array}$ \\
\hline Social support & 6 & .75 & $\begin{array}{l}\text { Do you expect support from the following people when } \\
\text { implementing Long Live Love? [governing body, colleagues } \\
\text { teaching the same and different subjects and the parent } \\
\text { association] } \\
\text { ( } 1=\text { no, certainly not, } 5=\text { yes, certainly) }\end{array}$ \\
\hline Self-efficacy & 12 & .76 & $\begin{array}{l}\text { I am able to create a safe atmosphere in the classroom where } \\
\text { students feel safe to openly talk about sex and relationships. } \\
\text { ( } 1=\text { no, certainly not, } 5=\text { yes, certainly) }\end{array}$ \\
\hline \multicolumn{4}{|l|}{ Interactive context } \\
\hline Student response & 3 & .63 & $\begin{array}{l}\text { Indicate how students generally respond to Long Live Love: } \\
\text { [interested, shy, positively] } \\
\text { (1= not at all, } 7=\text { yes, totally) }\end{array}$ \\
\hline
\end{tabular}


Table 1. (continued)

\begin{tabular}{|c|c|c|c|}
\hline & Items & a & Exemplary items (response scales) \\
\hline \multicolumn{4}{|l|}{ Implementation behavior } \\
\hline $\begin{array}{l}\text { Completeness- calculated as } \\
\text { 'Percentage of the program } \\
\text { (i.e. learning activities) being } \\
\text { implemented' (i.e. Slearning } \\
\text { activities/19*100) }\end{array}$ & 1 & & $\begin{array}{l}\text { Did you cover this (learning activity)? } \\
(1=\text { yes, } 0=\text { no })\end{array}$ \\
\hline Fidelity & 1 & & $\begin{array}{l}\text { How did you implement the new LLL program? } \\
\text { ( } 1=\text { I reviewed the program and only selected a few ideas for my } \\
\text { SRH lessons, } 2=\text { I reviewed the program and selected many ideas } \\
\text { for my SRH lessons, } 3=\text { I used the program as a guideline for my } \\
\text { lessons and delivered some lesson suggestions according to the } \\
\text { teacher manual, } 4=\text { I followed the guidelines of the program } \\
\text { as closely as possible and delivered most lesson suggestions } \\
\text { according to the teacher manual, } 5=I \text { delivered all lesson } \\
\text { suggestions for the LLL program exactly according to the teacher } \\
\text { manual.) }\end{array}$ \\
\hline
\end{tabular}

\section{RESULTS}

\section{Participants flow}

Of the 115 schools approached, a total of 45 schools (39\%), including 112 teachers agreed to participate in the pilot implementation of the revised LLL and the evaluation of their experience with implementing LLL. Teachers' non-willingness to participate was predominantly due to sexual education already having been provided in the school and lack of time. The schools were randomly assigned to either the waiting-list control group ( $\mathrm{N}=20$ schools, including $\mathrm{N}=46$ teachers) or the e-coach intervention group ( $\mathrm{N}=$ 25 schools, including $\mathrm{N}=66$ teachers). Two schools (one from each condition, including 6 teachers) withdrew before the start of the pilot implementation of LLL due to internal organisational changes leaving $\mathrm{N}=43$ schools ( $N=106$ teachers) at baseline. The baseline (T0) the survey was completed by 83 teachers (78\%; $N=50$ in the intervention group and $\mathrm{N}=33$ in the control group) from 43 schools. Non-response was mainly due to lack of time. Follow-up measurement (T1) was completed by 67 teachers ( $80 \%$ of those completing T0; $\mathrm{N}=41$ in the intervention group) from 38 schools. Drop out at T1 $(\mathrm{N}=16)$ was also mainly caused by lack of time. See Figure 1 for school allocation and participant flow. A drop-out analysis, accounting for teachers' background characteristics, indicated no significant differences between teachers who did versus those who did not drop out (t's $\left.\leq-0.36 ; P^{\prime} s \geq .07\right)$. 


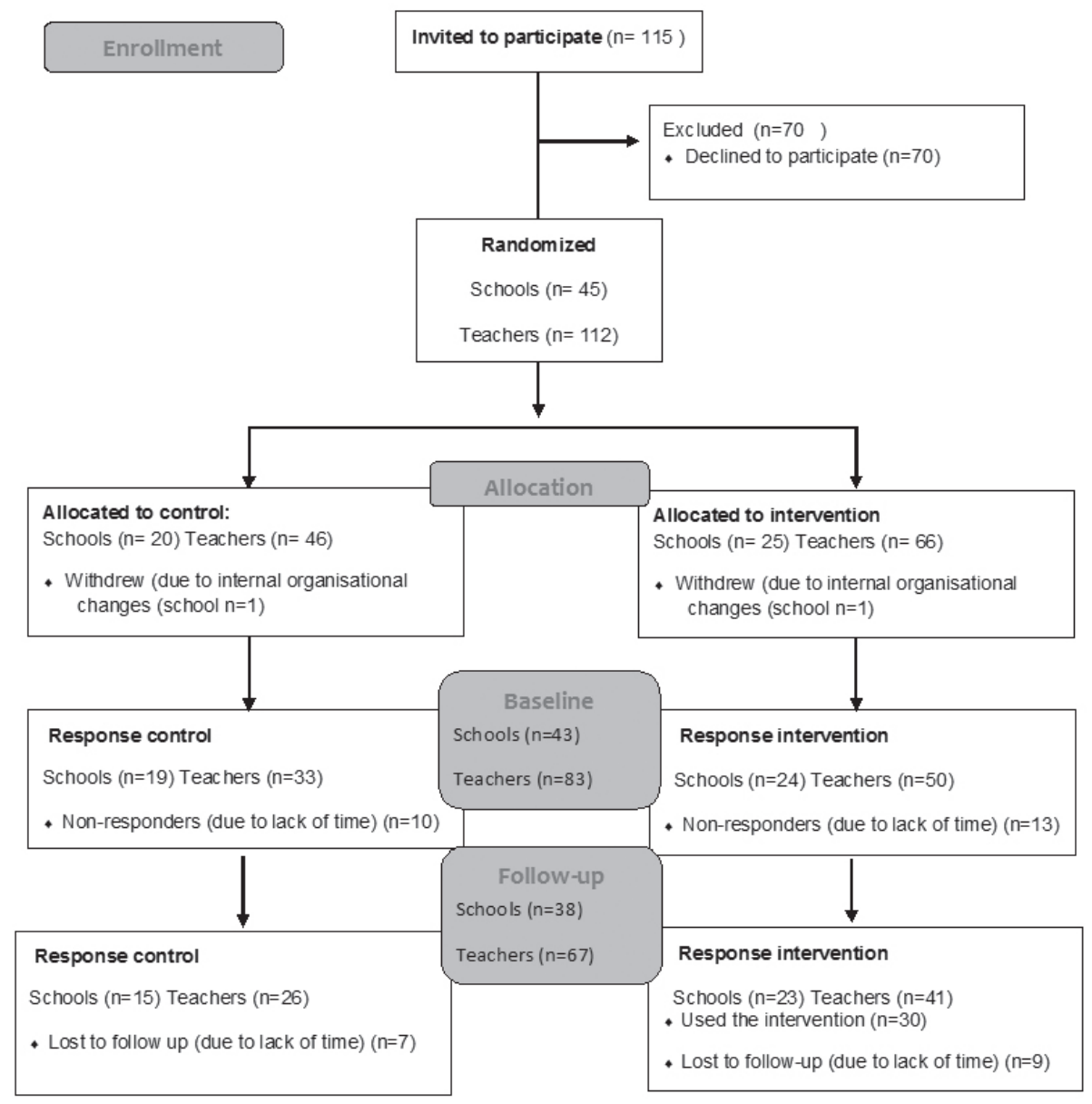

Figure 1. School allocation and participant flow. 


\section{Participants}

Of the 83 teachers participating in the baseline questionnaire, 53 were female (64\%) and $70 \%$ were biology teachers. The other teachers either taught the subject care $(n=19$; $22.89 \%$ ) or citizenship $(n=6 ; 7.23 \%)$. The mean age was 43 years ( $r a n g=24-63$, median $=44, S D=11.38$ ). Years of teaching experience ranged from 1 to 39 years (median $=11$; $\mathrm{SD}=10.43$ ), while years of experience teaching sexual education ranged from 0 to 35 (median $=7 ; \mathrm{SD}=7.91)$. Teachers generally felt fairly experienced in teaching sexual and reproductive health $(\mathrm{SRH})(\mathrm{M}=4.63 ; \mathrm{SD}=1.70)$, had a positive attitude towards teaching SRH ( $M=5.90 ; S D=0.83)$, had a positive attitude towards reflecting on their own SRH teaching methods $(M=5.94 ; S D=0.98)$ had a positive attitude towards teaching $S R H$ (5.87; $\mathrm{SD}=0.87)$ and a permissive sexual morality $(M=2.33 ; \mathrm{SD}=0.58)$. Teachers expressed a limited need for support in providing SRH lessons $(M=3.19 ; S D=1.04)$. One third of the teachers had experience with the previous LLL program, ranging from 1 to 10 years ( mean $=4.57$; median $=4 ; \mathrm{SD}=2.31$ ). No differences could be observed at baseline, between the intervention and control group. See Table 2 .

\section{Effects of e-coaching on (determinants of) implementation of LLL}

Overall, teachers reported completing on average $73 \%$ of the LLL program (range $=$ $37 \%-98 \%$ ) and $43 \%$ of teachers reported implementing the program largely conform the guidelines in the teacher manual $(M=3.46 ; S D=0.75)$. Only $6 \%(n=4)$ implemented LLL exactly conform the guidelines in the teacher manual. No significant difference was found in completeness $(p=.60$; Regression weight $=-2.12(3.99) ; 95 \%$ C.I. $=-10.26,6.02$; Cohen's $d=.17$ ) or fidelity ( $p=.67$; Regression weight $=.09(.21) ; 95 \%$ C.I. $=-0.33,0.51$; Cohen's $d=.14$ ) between teachers in the control group as compared to teachers in the e-coaching group, with small effect sizes (.17 and .14 respectively). See Table 3.

Also, no significant time $\mathrm{x}$ group interaction effect was found for the determinants of implementation behavior ( $\left(t^{\prime} s ; \leq 0.69 ; P^{\prime} s \geq .22\right.$ ). Some medium effect sizes were observed for social support $(\mathrm{d}=.30)$ and subjective norms $(\mathrm{d}=.33)$. However, the mean scores do not really show a potential trend in the expected direction. See Table 4.

Based on the survey, it turned out that of the 41 teachers in the intervention group, 30 actually visited the website (75\%). When comparing the 30 teachers who made actual use of the e-coaching website with the 37 teachers who did not, still no significant differences were found in completeness ( $P=.54$; Regression weight $=2.21$ (3.62); $95 \%$ C.I. for $B=-5.03,9.46)$ or fidelity of $\operatorname{LLL}(P=.74$; Regression weight $=0.06(.19) ; 95 \%$ C.I. for $B=-0.32,0.44)$. No significant differences were found between determinants either (t's $\leq 0.08 ; P^{\prime} s \geq .29$ ). 
Table 2. Demographic variables of teachers at T0.

\begin{tabular}{lccc}
\hline & \multicolumn{3}{c}{ Mean (SD): Baseline (T0) } \\
\cline { 2 - 4 } Demographic variables - teachers (range) & $\begin{array}{c}\text { Total } \\
(\mathbf{N}=\mathbf{8 3})\end{array}$ & $\begin{array}{c}\text { c-Group } \\
\text { (N= 33) }\end{array}$ & $\begin{array}{c}\text { I-Group } \\
\text { (N= 50) }\end{array}$ \\
\hline Age (23/64) & $43.11(11.38)$ & $42.97(11.30)$ & $42.94(11.59)$ \\
Years of teaching experience (1/39) & $14.37(10.43)$ & $13.67(10.86)$ & $14.60(10.08)$ \\
Years teaching SRH (0/35) & $9.20(7.91)$ & $9.61(7.73)$ & $9.12(8.09)$ \\
Experience teaching SRH (1/7) & $4.63(1.70)$ & $4.85(1.64)$ & $4.50(1.73)$ \\
Years teaching LLL (1/10) & $4.80(2.54)$ & $4.44(2.13)$ & $5.21(2.97)$ \\
Sexual morality (1/5) & $2.33(0.58)$ & $2.28(0.61)$ & $2.38(0.56)$ \\
Attitude towards teaching SRH (1/7) & $5.87(0.87)$ & $5.86(0.85)$ & $5.87(0.88)$ \\
Attitude towards reflecting on own SRH teaching methods (1/7) & $5.94(0.98)$ & $6.03(0.96)$ & $5.86(1.02)$ \\
Perceived need for support in providing SRH (1/5) & $3.18(1.04)$ & $3.36(.90)$ & $3.08(1.12)$ \\
\hline
\end{tabular}

Table 3. Direct effect of e-coaching on teachers' implementation behavior.

\begin{tabular}{lccccccc}
\hline $\begin{array}{l}\text { Implementation } \\
\text { behavior }\end{array}$ & $\begin{array}{c}\text { C-Group } \\
\text { Mean (SD) } \\
\mathbf{N}=\mathbf{2 6}\end{array}$ & $\begin{array}{c}\text { I-Group } \\
\text { Mean (SD) } \\
\mathbf{N = \mathbf { 4 1 }}\end{array}$ & $\begin{array}{c}\text { Regression } \\
\text { weight B (S.E.) }\end{array}$ & Sign. & $\mathbf{d}^{\text {a }}$ & $\mathbf{9 5 \% \text { C.I. for B }}$ \\
\hline Completeness LLL (\%) & $73.85(13.84)$ & $72.35(14.54)$ & $-2.12(3.99)$ & .60 & .17 & $-10.26 ;$ & 6.02 \\
Fidelity LLL (1/5) & $3.38(0.70)$ & $3.51(0.78)$ & $0.09(0.21)$ & .67 & .14 & $-0.33 ;$ & 0.51 \\
\hline
\end{tabular}

a. Effect sizes were calculated as $\beta / \sqrt{ }$ var, where $\beta$ is the estimated group effect at $\mathrm{T} 1$ and var is the residual outcome variance at that time point.

Table 4: Effect of e-coaching on determinants of implementation behavior.

\begin{tabular}{|c|c|c|c|c|c|c|c|c|c|}
\hline \multirow{3}{*}{$\begin{array}{l}\text { Determinants } \\
\text { Teacher benefits }\end{array}$} & \multicolumn{2}{|c|}{ Pre-test } & \multicolumn{2}{|c|}{ Post-test } & \multirow[b]{2}{*}{$\begin{array}{c}\text { Regression } \\
\text { weight B } \\
\text { (S.E.) }\end{array}$} & \multirow[b]{2}{*}{ Sign. } & \multirow[b]{2}{*}{$d^{a}$} & \multirow{2}{*}{\multicolumn{2}{|c|}{$\begin{array}{c}95 \% \text { C.I. } \\
\text { for B }\end{array}$}} \\
\hline & $\begin{array}{c}\text { C-Group } \\
\text { Mean (SD) } \\
\mathrm{N}=\mathbf{2 6}\end{array}$ & $\begin{array}{c}\text { I-Group } \\
\text { Mean (SD) } \\
\mathbf{N}=\mathbf{4 1}\end{array}$ & $\begin{array}{c}\text { c-Group } \\
\text { Mean (SD) } \\
\mathbf{N}=\mathbf{2 6}\end{array}$ & $\begin{array}{c}\text { I-Group } \\
\text { Mean (SD) } \\
\mathbf{N}=\mathbf{4 1}\end{array}$ & & & & & \\
\hline & $3.61(0.56)$ & $3.57(0.64)$ & $3.21(0.71)$ & $3.08(0.49)$ & $-.05(0.16)$ & .77 & .15 & -.36 & 0.26 \\
\hline Subjective norms & $4.08(0.53)$ & $4.03(0.47)$ & $4.24(0.55)$ & $4.09(0.54)$ & $-.08(0.15)$ & .60 & .33 & -.38 & 0.22 \\
\hline Social support & $4.17(0.44)$ & $4.14(0.57)$ & $4.26(0.55)$ & $4.30(0.55)$ & $.09(0.15)$ & .54 & .30 & -.20 & 0.38 \\
\hline Self-efficacy & $4.11(0.33)$ & $4.07(0.39)$ & $4.18(0.47)$ & $4.16(0.42)$ & $-.02(0.11)$ & .87 & .10 & -.23 & 0.19 \\
\hline Student response & $5.71(0.77)$ & $5.51(1.04)$ & $5.37(0.99)$ & $5.17(0.98)$ & $.03(0.28)$ & .92 & .06 & -.53 & 0.58 \\
\hline
\end{tabular}

a. Effect sizes were calculated as $\beta / \sqrt{ }$ var, where $\beta$ is the estimated group difference at $\mathrm{T} 1$ and var is the residual outcome variance at that time point. 


\section{DISCUSSION}

An e-coaching intervention was systematically developed to stimulate fidelity and completeness of use of the revised secondary school-based sex education program'Long Live Love' (LLL) in the Netherlands. The aim was to improve teachers' implementation behavior through self-reflection and skills development. The E coach was part of a broader implementation strategy that included a teacher training from the MHS prior to implementation.

Despite e-coaching being systematically developed, and with the input of experienced teachers, e-coaching was not found to be effective in changing teachers'implementation behavior or its determinants. In general, teachers implemented the new LLL program moderately during the pilot study. It is often difficult to prove the effectiveness of interventions or implementation strategies, even if they are solidly grounded in theory and evidence (Bartholemew et al., 2016).

Several factors might explain the lack of effect of e-coaching on implementation of LLL. Firstly, the study design itself may have had some flaws. Our study was an RCT design and included a baseline measure and post-test, yet the number of participants that completed both surveys was relatively low, despite the dropout rate being low. This could have resulted in a lack of power to find significant effects if present and affected the generalizability of the results. Moreover, a 'ceiling effect' could be present due to the implementation grade of teachers participating in the study already being high, making it difficult to improve using the e-coaching intervention. Finally, the teachers who agreed to participate in the study may have been a biased sample of motivated, experienced teachers who were already capable of delivering LLL successfully.

Secondly, for an intervention to have an effect, it is important that the intervention is used and positively perceived. By not being used or insufficiently used by teachers, e-coaching is unlikely to have an effect (Domitrovich et al., 2008; Forman et al., 2009; Payne, 2009). Despite being designed to support teachers in their implementation of LLL, the website itself also needed to be effectively implemented. Teachers were perhaps not motivated to use the website due to their extensive experience in teaching sexual and reproductive health $(\mathrm{SRH})$. Additionally, the broader implementation strategy developed to inform teachers about the e-coaching website was perhaps not optimally utilized and could potentially be optimized to increase use of e-coaching by teachers. For example, in this study not all MHS's provided a training and not all teachers who were offered a training accepted it. Motives for teachers' use or non-use of e-coaching need to be further explored as well as means to increase use of the website by teachers. 
Taking contextual factors as well as individual factors into consideration remains important when stimulating implementation (Schutte et al., 2016; Bartholemew et al., 2016; Durlak \& DuPre, 2008).

Lastly, the intervention itself may have been suboptimal. The e-coach was aimed at determinants of completeness and fidelity but may not have addressed the exact needs of the target population or been able to increase teachers' awareness of the importance of completeness and fidelity or did not address completeness and fidelity sufficiently or adequately. In developing e-coaching, program developers were already aware of the challenges involved: (1) teachers did not see their suboptimal implementation behavior as problematic and (2) teachers expressed a minimal need for coaching during the interviews in the needs assessment phase of program development (Schutte et al., 2016). The developers attempted to address these challenges in the development of e-coaching by using an unobtrusive coaching technique and stimulating self-reflection, yet the question remained whether this would be successful or not. The e-coach intervention may have been unable to change teachers' perceived need for coaching or change their awareness of their suboptimal implementation behavior with regard to completeness and fidelity, which may be linked to a lack of effect. This reemphasizes the importance of having a need for coaching or a desire for change prior to behavioral change (Parsloe \& Leedham, 2009). Means of stimulating teachers to use the website need to be explored.

Although completeness and fidelity of program delivery are crucial to the effectiveness of the program, teaching quality SRH lessons encompasses other teacher classroomrelated skills, such as creating a safe and trusted environment, which form the conditions for providing these lessons. Other studies on providing sexual education have also highlighted the importance of creating a safe environment when teaching this subject for optimal results (Vanwesenbeeck et al., 2015; Schaalma, Abraham, Gillmore, \& Kok, 2004). 'Teaching well' is thus more than completeness and fidelity. Therefore, in stimulating implementation of SRH programs, program developers should focus on enhancing completeness and fidelity as well as supporting teachers in creating the classroom conditions that enable quality delivery of SRH lessons, as e-coaching has attempted to do. 


\section{CONCLUSION}

The lack of effect of e-coaching does not insinuate that web-based coaching in itself is an ineffective strategy to promote fidelity and completeness of program implementation but in its current form, e-coaching may not have been ideal or the optimal intervention to achieve completeness and fidelity of LLL specifically. In order to further understand why e-coaching had no effect and how it could potentially be improved, a process evaluation is required to find out how and to what extent teachers made use of the website, how they appreciated it and what factors affected teachers use of the website.

\section{ABBRE VIATIONS}

ERCPN: Ethical Review Committee of Psychology \& Neuroscience

Dol: Diffusion of Innovations theory

IM: Intervention Mapping

LLL: Long Live Love

MHS: Municipal Health Services

SCT: Social Cognitive Theory

SRH: Sexual Reproductive Health

TPB: Theory of Planned Behavior 


\section{CHAPTER 6}

\section{Process evaluation of a Web-based}

\section{coaching intervention to support}

implementation of sex education

\section{among secondary-school teachers}

Chapter submitted as:

Schutte, L., Mevissen, F.E.F., Meijer, S., Paulussen, T., van Empelen, P. (2017). Process evaluation of a Web-based coaching intervention to support implementation of sex education among secondary school teachers. Journal of Medical Internet Research. 


\section{ABSTRACT}

Background: The quality of implementation is an important component for the effectiveness of behavioral change interventions. In school settings, teachers' support during implementation is often limited and appears to fall short when attempting to preserve completeness and fidelity in program delivery. With the aim to improve completeness and fidelity of teachers' delivery of a sexual health promoting intervention ('Long Live Love') (LLL) in secondary education, a web-based e-coach was developed ('lesgevenindeliefde.nl'-'teachinglove.nl'). An effect- and a process evaluation were performed to evaluate the effectiveness of the e-coach in influencing teachers' implementation as well as to evaluate the teachers' use and appreciation of the e-coach. It appeared that the e-coach did not have an effect on teachers' implementation behavior. This paper reports on the process evaluation including teachers' subjective evaluation of the e-coach and reasons for (not) using the e-coach.

Methods: A survey questionnaire $(\mathrm{N}=67)$ and additional interviews $(\mathrm{N}=20)$ were conducted among teachers who had had access to the e-coach, in which appreciation, use and (motives for) use of the e-coach were investigated.

Results: The e-coaching website appeared well appreciated by teachers and was considered instrumental during implementation of LLL. However, the e-coach was used minimally and not as intended by program developers. This suboptimal use of e-coaching is likely the reason why it did not have an impact on (the determinants of) teachers' implementation behavior. Impeding factors for using the e-coach were a lack of time, the introduction of two innovations at once ( $L L L+$ e-coaching), and low personal relevance of the website because of unmet expectations, not finding new information on the website due to extensive experience teaching SRH and no perceived need for coaching. Factors that stimulated (continued) use of e-coaching were a positive attitude towards LLL, a positive subjective evaluation of the e-coach, expecting to find student materials on the website and receiving an MHS teacher training prior to implementing LLL.

Conclusion: The e-coach was not used at all or not used optimally by teachers. This is likely the main reason why it did not have impact. Low personal relevance was the main reason why the e-coach was not used by teachers, along with the external factors of lack of time and the introduction of two innovations at once. The lack of personal relevance of the e-coach resulted largely from teachers not feeling a need for coaching, thereby not using the website or discontinuing use altogether. This lack of need for coaching was already established in the needs assessment phase of the e-coach development. 
It therefore appears that the e-coach was unable to change teachers' perceived need for coaching. In hindsight, we realize, as a result of this process evaluation, that a discrepancy exists between teachers and e-coach developers on the perceived need for coaching which is related to a different interpretation regarding quality of teaching. For teachers, 'correct' program delivery and thus good teaching is related to having sufficient didactic skills. For program developers, however, 'correct' program delivery is related to implementation with completeness and fidelity. It seems teachers may first have to understand the importance of completeness and fidelity in the implementation of LLL and consider it a component of good teaching, prior to behavioral change through e-coaching. The e-coaching intervention itself will need to be optimized to address the importance of completeness and fidelity, and contain more tools aimed at enhancing the completeness and fidelity of LLL specifically. Use of the e-coach needs to be stimulated by increasing the personal relevance of the website and utilizing the broader implementation strategy. 



\section{INTRODUCTION}

An important factor in the effectiveness of behavioral change interventions is the quality of its implementation: an intervention that is implemented completely and according to its' guidelines is more likely to be successful in changing the target groups' determinants and behavior than programs that are not implemented fully (Little et al., 2015; Lendum \& Humphrey, 2012; Durlak \& Dupre, 2008). Implementation is, however, often inadequate and mostly overlooked or insufficiently considered when planning for and developing behavioral change interventions (Bartholemew et al., 2016). There is a need for greater attention to the quality of implementation (Fagan \& Mihalic, 2003). Implementation is not an automatic process. Therefore, an implementation strategy needs to take into account awareness, decision to use, actual, complete and correct use and maintained use, respectively.

In school-based sexual health promotion, teachers are key to program delivery. Implementation is, however, often sub- optimal (Bessems et al., 2011; Peters et al., 2009; Schutte et al., 2014); programs are not being implemented completely or with sufficient fidelity to produce measurable outcomes (Gottfredson \& Gottfredson, 2002; Hoekstra et al., 2009).

Teachers appear to be in need of support to enable them to put the innovation into practice (Durlak \& DuPre, 2008; Rohrbach et al., 2006). Most support has been invested in promoting teachers' awareness and adoption of new interventions but less is known about the best way to support teachers in the implementation phase (Bessems et al., 2011; Domitrovich \& Greenberg, 2000; Fagan \& Mihalic, 2003; Durlak, 1998). This is especially true for the particularly challenging school-based sex education programs which address the sensitive subject of sexuality. Currently, this support is limited to providing practical support in the form of program material, including teacher manuals with practical information on the content of the lessons and on how to deliver such lessons. In some cases, providing support prior to implementation in the form of training often equips teachers with skills for correct implementation but it is not enough (Dusenbury et al., 2003). More in-depth coaching focusing on determinants of implementation such as self-efficacy and social support to enhance completeness and fidelity is lacking (Van de Bongardt et al., 2009; Bartholemew et al., 2016; Hansen et al., 2009; Rohrbach et al., 2010; Kam et al., 2003; Mihalic et al., 2008; Farmer-Dougan et al., 1999; Jones et al., 1997; Mortenson \& Witt, 1998; Noell et al., 1997; Witt et al., 1997; Hesselink et al., 2009). It remains important to provide teachers with more personal assistance and ongoing support and consultation during the process of putting an innovation into practice (Schutte et al., 2014; Kramer et al., 2000; Rohrbach et al., 2006; 
Durlak, 1998). Coaching would provide more than just a one-time training, namely assistance during real-life implementation situations, with a longitudinal character in that teachers could receive assistance when convenient and relevant to them (Hann \& Weiss, 2005).

\section{Implementation of the 'Long Live Love' program}

In the Netherlands, Long Live Love (LLL) has been proven effective and is widely used in Dutch secondary education (Schaalma et al, 1996; Schutte et al, 2014). LLL is a teaching pack about relationships and sexuality for the second year of secondary education (ages 13-15) and has recently been revised. The evaluation of the previous version of LLL showed that implementation was sub-optimal: teachers indicated that they used the program components selectively, adjusted it with their own additions, delivered the program incompletely, made limited use of the teacher manual and did not comply to the prescribed stages in teacher planning, preparation and evaluation (Schutte et al., 2014; Schutte et al., 2016). This suboptimal implementation behavior may lead to reduced program effectiveness (Bessems et al., 2014; Peters et al., 2009). Teachers, however, did not perceive this behavior as problematic and therefore expressed minimal need for coaching or behavioral change. This revealed a discrepancy between actual need for coaching, according to program developers and need for support as perceived and articulated by teachers in delivering LLL; as program developers, we aim for completeness and fidelity of program implementation and perceive teachers' teaching behavior as suboptimal in this regard. As implementers, teachers, however do not perceive their teaching behavior as problematic and thus feel no need for coaching in teaching SRH generally or LLL specifically. Teachers did, however, acknowledge some difficulties encountered when providing sex education, that according to more experienced teachers, were predominantly faced by their less experienced colleagues. Being unable to deal with these difficulties, such as an inability to create a safe atmosphere in the classroom, could become a barrier for completeness and fidelity of LLL because these form the prerequired conditions for delivery of SRH lessons generally (Schutte et al., 2016).

\section{E-coach}

In order to improve completeness and fidelity of program delivery, teachers need awareness of their own (suboptimal) implementation behavior, support in delivering the program with completeness and fidelity and in coping with potentially difficult situations to create the preconditions required to deliver $\mathrm{SRH}$ lessons. An e-coaching 
intervention ('lesgevenindeliefde.nl' - 'teachinglove.nl') was systematically developed for that purpose (Schutte et al., 2016). The goal was to improve teachers' implementation behavior through self-reflection and skills development (Schutte et al., 2016).

The E-coach was part of a broader implementation strategy that also included protocols to promote adoption and continuation of LLL, a pre-implementation teacher training delivered by the Municipal Health Services (MHS) and a teacher manual to enhance implementation.

\section{Evaluation of e-coaching}

The e-coaching intervention was implemented simultaneously with the revised LLL program. To measure the effectiveness of the e-coach on teachers' implementation behavior, half of the teachers implementing the revised LLL were invited to make use of the web-based e-coach, next to the teachers' manual. The other half of the teachers only received the teachers' manual. The E-coach was not found to have an effect on teachers' implementation behavior. See Schutte et al., (2017) for more details on the effect evaluation.

This paper focuses on the process evaluation of the e-coaching, to further understand why e-coaching had no effect by examining how and why the innovation was appreciated and used by teachers and understand how the innovation could potentially be improved. More specifically, the research questions were:

$R Q 1$. How did teachers perceive the instrumentality, usability and likeability of the e-coaching website?

$R Q 2$. To what extent was the e-coaching website used by teachers?

$R Q$ 3. What were motives for (continued) use or non-use of the e-coaching website?

The extent to which the website is used and appreciated by teachers will have an impact on implementation quality (Hansen et al., 2009). Website use or intention to use online interventions is, in turn, influenced by attitude towards the intervention, which in turn is predicted by perceived usefulness and perceived ease of use of the intervention (Pijpers et al., 2002). 


\section{METHODS}

The e-coaching website was evaluated by quantitative and qualitative methods.

\section{Design}

A clustered randomized controlled trial (e-coaching vs control) was conducted, with a baseline assessment (T0) and follow up (T1) two weeks after completing the delivery of the LLL program. Teachers in both groups received the LLL program, a teacher manual and the option to receive a teachers' training from the MHS. Only teachers in the intervention group were additionally offered access to the e-coaching website with a personal user name and password sent to them by e-mail. Their teacher manual also contained references to the e-coach website and also their teachers' training included information on the e-coaching.

\section{Interventions}

\section{E-coaching}

The Intervention Mapping (IM) protocol was used to systematically develop the webbased e-coach. For a detailed description of the development and content of the website see Schutte et al., (2016). E-coaching was ultimately aimed at promoting fidelity and completeness of LLL in a stepwise process, namely by: i) stimulating teachers to reflect on their Sexual and Reproductive Health (SRH) teaching, ii) encouraging teachers to deliver all six LLL lessons (completeness) according to the guidelines in the teacher manual (fidelity) and iii) supporting teachers to anticipate and effectively cope with the most common difficulties encountered when providing $\mathrm{SRH}$.

These common difficulties were derived from a previously conducted needs assessment (Schutte et al., 2016) and included 1) creating a safe and secure atmosphere for the provision of SRH lessons, 2) teaching $\mathrm{SRH}$ without shame influencing the quality of the lessons, 3) protecting boundaries in sharing personal information and questions between teachers and students and among students themselves, 4) identifying personal problems of students, 5) integrating social media in SRH lessons, 6) dealing with diversity (e.g. sexual inclination, ethnicity, sexual experience).

With teachers' limited awareness of the implementation problem and related resistance to coaching in mind, an unobtrusive coaching technique was developed. This technique aimed at creating a need for coaching in teachers, without awakening resistance, by exposing them to difficulties experienced by other teachers they identified with, in 
the delivery of SRH. To create a 'need for coaching' or desire for 'behavioral change' meant creating awareness that their teaching behavior is not optimal when it comes to completeness and fidelity and that they need coaching in order to change this.

Teachers are expected to use the website during preparation of a LLL lesson and/or after they encountered particular difficult situations in their classes. A self-reflection tool was developed to enable teachers to reflect critically on their own implementation behavior and to create awareness of their need for additional support. Coaching can only start when teachers develop awareness of their needs and desire to improve their classroom performance (Parsloe \& Leedham, 2009). With the results of the self-reflection tool, teachers were referred to additional support found on website pages that were personally relevant to them. These included videos, stories, background information on the specific behavior, tips, FAQ, and the option to ask for support from other teachers. Teachers were also informed about completeness and fidelity by means of video, role model stories and tips. In addition, the e-coach included student materials meant to stimulate teachers to visit the e-coach.

\section{Teachers' training}

A training was offered by the Municipal Health Services (MHS) to teachers in both the e-coach intervention group and the control group. The training introduced the LLL program to teachers and aimed to motivate them to use the program and use it as intended by enhancing teacher's knowledge, attitudes and skills regarding LLL and its delivery. Teachers in the intervention group received additional information during the training about the e-coaching website and were explicitly motivated by the MHS to use it during the pilot implementation of LLL.

\section{Recruitment \& Procedure}

From all the secondary schools in the Netherlands $(\mathrm{N}=610)$, a sample of $\mathrm{N}=115(19 \%)$ schools were invited to participate in the pilot implementation of LLL. They were randomly selected, after stratification according to region and education level. Schools that agreed to participate were randomly assigned to either the e-coaching intervention group or the control group. Teachers from the schools that agreed to participate and who taught SRH were invited by e-mail and telephone to follow the teacher training from the MHS in their region and to complete the surveys (T0 followed by $\mathrm{T} 1$ within two weeks of completing the LLL program). The baseline and post-test surveys took approximately 30 minutes to complete. Non-responders were reminded by e-mail and eventually by telephone 3 days after the initial deadline. 
Separate trainings were delivered to teachers in the e-coach intervention group vs. the control group, prior to LLL program implementation. In the end, more than half the teachers who participated in the baseline measures $(56.5 \% ; \mathrm{N}=47 ; \mathrm{n}=21$ in the control group; $\mathrm{n}=26$ in the intervention e-coach group) were actually offered a training by the MHS. Finally, 58.2\% ( $N=39 ; n=15$ control group; $n=24$ intervention e-coach group) of the participating teachers in the survey received a training from 14 different MHS'. The remaining teachers did not receive training, either because they refused the training as they felt there was no need or because the MHS in their region was not offering the training.

After filling out T0, teachers in both groups received the newly revised LLL program (a package including a student magazine, a student DVD, and a teacher manual) by post mail, which they could implement within the subsequent two months (approximately). Only teachers in the intervention group were at the same time given access to the e-coaching website and were invited to use it while implementing LLL. They were informed by e-mail that the website could support them in their implementation of LLL and on what the website had to offer (videos, role model stories, tips etc). Halfway during the pilot study, an e-mail reminded teachers in the intervention group to use the e-coaching website. Confidentiality was preserved throughout the study. All procedures in the study were approved by the authorized Ethical Review Committee of Psychology \& Neuroscience (ERCPN) at Maastricht University.

Participants for the interviews were recruited by e-mail, solely among teachers within the intervention group, who had completed and returned T1 $(\mathrm{N}=41)$. Teachers willing to participate were interviewed two weeks after completing the LLL program. Teachers were first asked questions based on recall of website use and were then asked to browse through the website, while seated behind a computer. The interviews lasted approximately 1 hour each.

\section{Measurements}

Demographics were measured at baseline (gender, years of teaching experience, years of experience teaching $\mathrm{SRH}$, sexual morality, need for support in providing $\mathrm{SRH}$ lessons etc) and some were included in the post-test survey (gender, age). This process evaluation further focuses solely on the intervention group, namely the post-test survey and interviews conducted with teachers in the e-coaching group. The posttest survey asked whether the teacher had attended the MHS teacher training and included measures of implementation behavior, namely completeness and fidelity, and its related determinants as considered in the effect evaluation (see Schutte et al., 2017, accepted for publication under review). In addition, teachers in the intervention 
group were specifically asked about their actual use (e.g. frequency and extent of use), intention of continued use of the e-coaching website and its' perceived instrumentality and usability, and motives for (continued) use of the website. To measure usability, perceived efficiency, effectiveness and enjoyment of the website were measured as aspects of the users' experience (Kassenaar, \& van Rijswijk, 2003; Sears \& Jacko, 2003). Efficiency is the ease with which users of the website can find information that they are looking for. Effectiveness is whether the website provides information that the user requires, and enjoyment is the experience of the user, for example, the extent to which the website is attractive to them. See Table 1 for an overview of measures included in this study.

The interviews were used to provide additional insight in teachers' use and evaluation of e-coaching as well as their motives for (continued) use or non-use of the coaching website. The interviews for the process evaluation were semi-structured and based on a topic list including topics such as impressions of the website, reasons for (continued) (non-)use of e-coaching and appreciation of the website. 
Table 1. Measures, example items and scale.

\begin{tabular}{|c|c|c|}
\hline & Items & Example items \# Scale \\
\hline \multicolumn{3}{|l|}{$\begin{array}{l}\text { Demographic variables - } \\
\text { Teacher }\end{array}$} \\
\hline Gender & 1 & $\begin{array}{l}\text { 'What is your gender?' } \\
\# 0=\text { female, } 1=\text { male }\end{array}$ \\
\hline Age & 1 & 'What is your age'? \\
\hline Teaching subject & 1 & $\begin{array}{l}\text { 'What subject do you teach?' } \\
\# 1=\text { biology, } 2 \text { = health care, } 3=\text { citizenship, } 4=\text { other }\end{array}$ \\
\hline Years of teaching experience & 1 & 'How many years have you been working in education?' \\
\hline Years teaching SRH & 1 & 'How many years have you been teaching SRH?' \\
\hline Experience teaching SRH & 1 & $\begin{array}{l}\text { 'How experienced are you in teaching SRH?' } \\
\# 1 \text { = very inexperienced, } 7=\text { very experienced }\end{array}$ \\
\hline $\begin{array}{l}\text { Perceived need for support } \\
\text { in providing SRH }\end{array}$ & 1 & $\begin{array}{l}\text { 'Do you need support in providing SRH lessons?' } \\
\# 1 \text { = no, certainly not, } 5=\text { yes, certainly }\end{array}$ \\
\hline $\begin{array}{l}\text { Attitude towards teaching } \\
\text { SRH }\end{array}$ & 6 & $\begin{array}{l}\text { 'Indicate what you think about teaching SRH: important, necessary, fun, difficult, } \\
\text { comfortable, competent' } \\
\# 1 \text { = not at all, } 7 \text { = yes, totally }\end{array}$ \\
\hline $\begin{array}{l}\text { Attitude towards reflecting } \\
\text { on own SRH teaching } \\
\text { methods }\end{array}$ & 1 & $\begin{array}{l}\text { 'Indicate what you think about reflecting on your own SRH teaching methods: important, } \\
\text { useful, good' } \\
\# 1=\text { not at all, } 7 \text { = yes, totally }\end{array}$ \\
\hline Use of previous LLL & 1 & $\begin{array}{l}\text { Have you used the previous LLL in the past for SRH lessons? } \\
\# 0=\text { no, } 1=\text { yes }\end{array}$ \\
\hline Years teaching LLL & 1 & 'For how many years have you been using Long Live Love?' \\
\hline Sexual morality & 5 & $\begin{array}{l}\text { 'Young people who have just met should not have sex' } \\
\# 1 \text { = strongly disagree, } 5=\text { strongly agree }\end{array}$ \\
\hline \multicolumn{3}{|l|}{ Curriculum related beliefs } \\
\hline Outcome beliefs: & 8 & $\begin{array}{l}\text { Measured as a weighted result of the teacher's perceived importance and perceived feasibility } \\
\left.\text { (i.e. } \Sigma f^{*} i / 8\right)\end{array}$ \\
\hline $\begin{array}{l}\text { perceived importance of } \\
\text { student learning outcomes }\end{array}$ & 8 & $\begin{array}{l}\text { 'How important is it to you that your students can communicate and negotiate about safe } \\
\text { sex with their partner?' } \\
\# 1=\text { not important at all, } 5=\text { very important }\end{array}$ \\
\hline $\begin{array}{l}\text { perceived feasibility of these } \\
\text { outcomes }\end{array}$ & 8 & $\begin{array}{l}\text { 'Do you expect to achieve that students can use condoms or anticonception correctly?' } \\
\# 1=\text { no, not at all, } 5=\text { yes, certainly }\end{array}$ \\
\hline Teacher benefits & 6 & $\begin{array}{l}\text { 'I gained insight in the sexuality experience of youngsters' } \\
\# 1 \text { = strongly disagree, } 5 \text { = strongly agree }\end{array}$ \\
\hline Instrumentality & 13 & $\begin{array}{l}\text { 'The LLL program provides sufficient guidelines to adequately provide SRH' } \\
\# 1=\text { completely disagree, } 5=\text { completely agree }\end{array}$ \\
\hline Subjective norms & 6 & $\begin{array}{l}\text { 'Do you think that the following people appreciate you using Long Live Love to provide } \\
\text { sexual education?' (principal, governing body, external consultants/health education } \\
\text { experts, students, colleagues teaching the same and colleagues teaching a different subject, } \\
\text { parents) } \\
\# 1=\text { no, certainly not, } 5=\text { yes, certainly }\end{array}$ \\
\hline Social support & 6 & $\begin{array}{l}\text { 'Do you expect support from the following people when implementing Long Live Love?' } \\
\text { (governing body, colleagues teaching the same and different subjects and the parent } \\
\text { association) } \\
\text { \# } 1=\text { no, certainly not, } 5=\text { yes, certainly }\end{array}$ \\
\hline Self-efficacy & 12 & $\begin{array}{l}\text { 'I am able to create a safe atmosphere in the classroom where students feel safe to openly } \\
\text { talk about sex and relationships' } \\
\# 1=\text { no, certainly not, } 5=\text { yes, certainly }\end{array}$ \\
\hline
\end{tabular}


Table 1. (continued)

\begin{tabular}{|c|c|c|}
\hline & Items & Example items \# Scale \\
\hline \multicolumn{3}{|l|}{ Information source - MHS } \\
\hline Teacher training offered & 1 & $\begin{array}{l}\text { 'Did you get offered a training by the MHS for the new LLL program in the Springtime?' } \\
\# 0=\text { no, } 1 \text { = yes }\end{array}$ \\
\hline Teacher training received & 1 & $\begin{array}{l}\text { 'Did you receive a training in the Springtime by the MHS for the new LLL program?' } \\
\# 0=\text { no, } 1 \text { = yes }\end{array}$ \\
\hline \multicolumn{3}{|l|}{ E-coaching } \\
\hline Used at all & 1 & $\begin{array}{l}\text { 'Did you visit the 'Lesgeven in de Liefde' website for teachers during your use of the new LLL } \\
\text { program?' } \\
\# 0=\text { no, never, } 1 \text { = yes }\end{array}$ \\
\hline Moment of use & 7 & $\begin{array}{l}\text { 'At which moments did you make use of the 'Lesgeven in de Liefde' website?' (during } \\
\text { preparation of each lesson, at the end of each lesson, I viewed everything on the website in } \\
\text { one go, as soon as I encountered a challenge during the provision of SRH, as soon as I had a } \\
\text { question, as soon as I got a reminder e-mail to go to the website, other) } \\
\# 0=\text { no, } 1 \text { = yes }\end{array}$ \\
\hline Extent of use website & 1 & $\begin{array}{l}\text { 'How did you make use of the 'Lesgeven in de Liefde' website? } \\
\# 1=\text { I only visited the homepage, } 2=1 \text { only viewed a few components of the website, } 3=1 \\
\text { viewed some components of the website several times, } 4=\text { I viewed all components of the } \\
\text { website }\end{array}$ \\
\hline Frequency of use & 1 & $\begin{array}{l}\text { 'How often did you make use of the 'Lesgeven in de Liefde' website during the use of the } \\
\text { new LLL program?' } \\
\# 1=1 \text { time, } 2=2-3 \text { times, } 3=4 \text { to } 5 \text { times, } 4=6 \text { or more times }\end{array}$ \\
\hline Actions & 10 & $\begin{array}{l}\text { 'What did you do on the 'Lesgeven in de Liefde' website?' (watched videos, read role model } \\
\text { stories, filled out the self-evaluation quiz, placed reactions, asked questions, viewed FAQ, } \\
\text { read background information on the challenges, read tips, downloaded worksheets) } \\
\# 0=\text { no, } 1=\text { yes }\end{array}$ \\
\hline Challenges viewed & 9 & $\begin{array}{l}\text { 'Which challenges did you view on the 'Lesgeven in de Liefde' website?' (none, creating a } \\
\text { safe atmosphere, teaching without shame, guarding boundaries, identifying problems of } \\
\text { students, integrating actual themes, dealing with homosexuality, gender- or experience } \\
\text { differences, discussion in the classroom, approach to providing lessons) } \\
\text { \# } 0=\text { no, } 1 \text { = yes }\end{array}$ \\
\hline $\begin{array}{l}\text { Intention to continue using } \\
\text { website }\end{array}$ & 1 & $\begin{array}{l}\text { If you provide SRH lessons next school year, will you make use of the 'Lesgeven in de Liefde' } \\
\text { website again?' Explain. } \\
\# 1=\text { no, certainly not, } 5 \text { = yes, certainly }\end{array}$ \\
\hline Usability website & 9 & $\begin{array}{l}\text { 'It is easy to find the information I need on the 'Lesgeven in de Liefde' website' } \\
\# 1=\text { strongly disagree, } 5=\text { strongly agree }\end{array}$ \\
\hline $\begin{array}{l}\text { Perceived instrumentality } \\
\text { website }\end{array}$ & 1 & $\begin{array}{l}\text { Was the website 'Lesgeven in de Liefde' helpful in preparing your LLL lessons?' } \\
\# 1=\text { no, certainly not, } 5=\text { yes, certainly }\end{array}$ \\
\hline \multicolumn{3}{|l|}{ Implementation behavior } \\
\hline Completeness & 1 & $\begin{array}{l}\text { 'Percentage of the program (i.e. learning activities) being implemented' (i.e. Elearning } \\
\text { activities } / 19^{*} 100 \text { ) }\end{array}$ \\
\hline Fidelity & 1 & $\begin{array}{l}\text { 'How did you implement the new LLL program?' } \\
\text { \# } 1 \text { = I reviewed the program and only selected a few ideas for my SRH lessons, } 2=1 \\
\text { reviewed the program and selected many ideas for my SRH lessons, } 3=\mid \text { I used the program } \\
\text { as a guideline for my lessons and delivered some lesson suggestions according to the } \\
\text { teacher manual, } 4=\text { I followed the guidelines of the program as closely as possible and } \\
\text { delivered most lesson suggestions according to the teacher manual, } 5=\mid \text { delivered all } \\
\text { lesson suggestions for the LLL program exactly according to the teacher manual. }\end{array}$ \\
\hline Continuation & 1 & $\begin{array}{l}\text { Do you intend on using LLL next school year for your sexual education lessons?' Explain. } \\
\# 1=\text { no, certainly not, } 5=\text { yes, certainly }\end{array}$ \\
\hline
\end{tabular}




\section{Analysis}

All quantitative data from the survey were analysed using SPSS24. Website use and perceived instrumentality and usability of the e-coaching website, were analysed using frequencies and descriptives. T-tests were used to check for significant differences in demographics between users and non-users of e-coaching within the intervention group. The effects of the MHS training (for the intervention group) on frequency of website use were analysed using multilevel regression analysis which included two levels: school and teacher. Correlations and multilevel regression were conducted to identify motives for continued use of e-coaching.

The interviews were transcribed and MAXQDA was used to code and group the qualitative data according to theme. In the results, both the qualitative and quantitative data will be presented simultaneously.

\section{RES ULTS}

\section{Participants flow}

Survey: A total of 43 schools with 83 teachers participated in the study. In the followup 38 schools participated; 23 in the e-coaching condition with 41 teachers, 15 in the control condition with 26 teachers. Non-response was mainly due to a lack of time. See Figure 1 for school allocation and participant flow.

Interview: Twenty teachers from the intervention group ( $\mathrm{N}=41,49 \%$ response rate) were interviewed after completing the LLL program. Reason for non-participation was a lack of time.

\section{Participants}

Survey: Of the 67 teachers participating in the follow-up questionnaire, 41 were in the intervention group. Of these 41 teachers, 31 were female (78\%) and $63 \%$ were biology teachers. The other teachers either taught the subject care $(n=13 ; 31.71 \%)$ or citizenship $(n=2 ; 4.89 \%)$. The mean age was 43 years ( $r a n g=24-61$, median $=45, S D=11.50)$. Years of teaching experience ranged from 1 to 38 years (median $=13 ; S D=9.85$ ), while years of experience teaching sexual education ranged from 0 to 35 (median $=10 ; S D=8.28$ ). Teachers generally felt fairly experienced in teaching sexual and reproductive health $(\mathrm{SRH})(\mathrm{M}=4.59 ; \mathrm{SD}=1.76)$, had a positive attitude towards teaching SRH ( $a=.82 ; \mathrm{M}=$ 5.88; $\mathrm{SD}=0.92)$, had a positive attitude towards reflecting on their own $\mathrm{SRH}$ teaching methods $(M=5.82 ; S D=1.06)$ and a permissive sexual morality $(a=.62 ; M=2.34 ; S D=.60)$. 
Teachers also expressed a limited perceived need for support in providing SRH lessons $(M=2.95 ; S D=1.14)$. One third of the teachers had experience with the previous LLL program, ranging from 1 to 10 years ( mean $=5.08$; median $=3.50 ; \mathrm{SD}=3.09$ ).

Interviews: The interviewed teachers $(\mathrm{N}=20)$ were from 18 different schools from diverse parts of the country. There were 13 women and 7 men with 1 to 20 years of teaching experience, ranging from 23 to 61 years old. They had all implemented LLL. Of the 20 teachers interviewed in the intervention group, 10 had visited the website at least once. The remaining 10 teachers did not visit the website at all and solely implemented LLL.

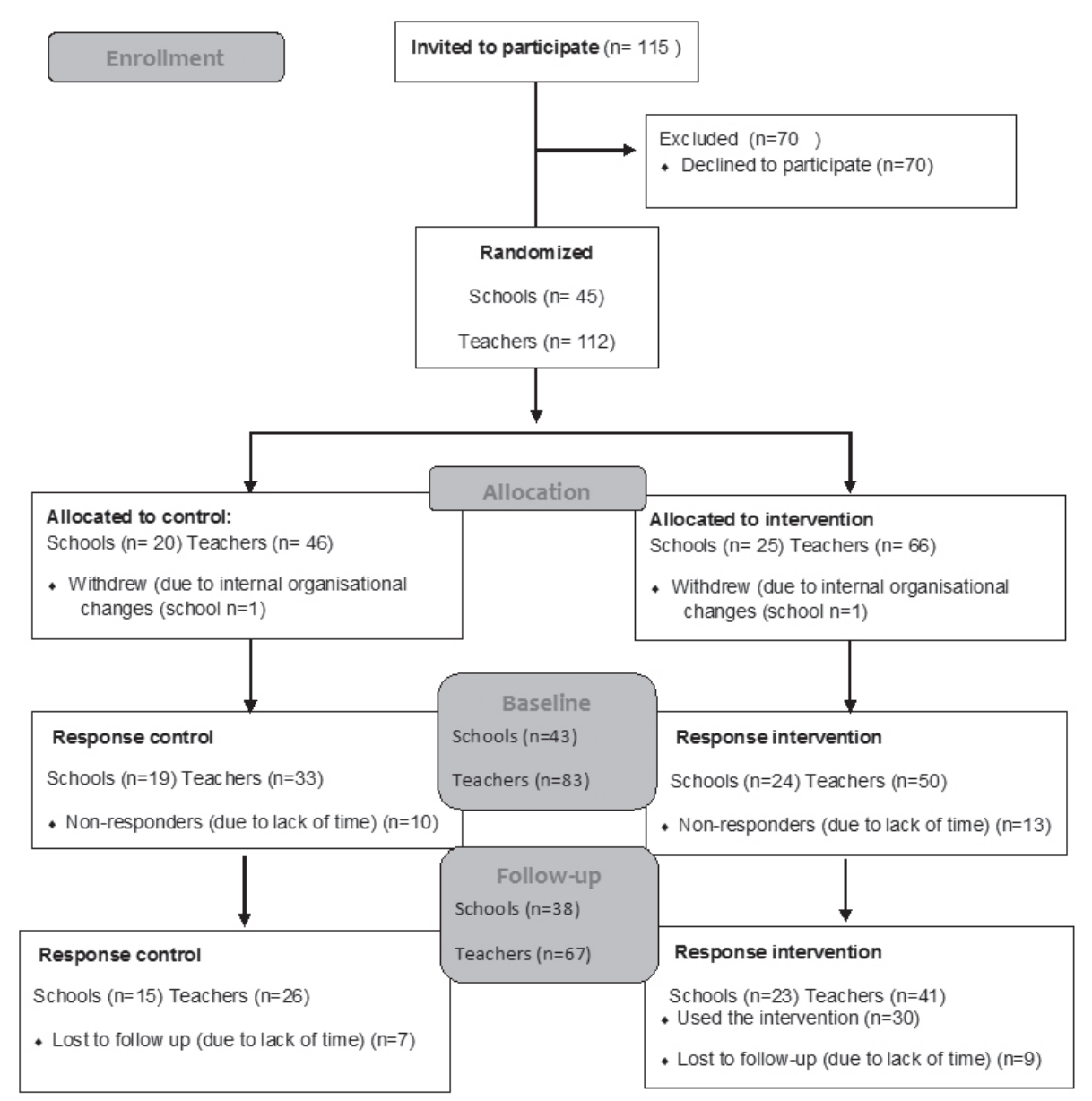

Figure 1. School allocation and participant flow. 


\section{Evaluation of e-coaching}

Subjective evaluations of the website's usability and perceived instrumentality for preparing LLL lessons were moderately positive $(M=3.72$; $S D=0.52$ respectively $M=3.24 ; S D=1.24)$. These findings are supported by the interviews in which teachers described the website as well structured, clear and easy to navigate:

"It is easily accessible and fast. You can easily find answers to your questions. Yes so, to me it's just fine." (Teacher, 27 years, female).

Teachers also liked the accessibility of the website: it is there to support them if ever they would need it and they found the website instrumental to the LLL program:

"I like the fact that the website is there to complement the LLL program." (Teacher, 35 years, female).

"I felt like, if I wanted to see the website again, I could anytime and I liked that. Imagine I encounter a difficulty, then I feel there is support via the website. Then I can have a look at how other teachers handled it." (Teacher, 52, female).

Impressions of the website were positive, especially the role-model stories and videos. Teachers especially appreciated the aspect of learning from other teachers sharing their experiences on the e-coaching website:

"Yes, it's useful to see with what attitude and experience other teachers deal with these difficulties. By reading about and watching them, you keep these possibilities at the back of your mind." (Teacher, 52 years, female).

Furthermore, the interviewed teachers evaluated the website content as credible and recognisable. Teachers also recognised the difficulties represented in the website as (potentially) encounterable when providing sexual education.

\section{Use of e-coaching}

Of the 41 teachers in the intervention group invited to use the e-coach, 30 actually visited the website $(75 \%)$, of which the majority did so 2 to 3 times $(n=20 ; 66.7 \%$ ). Most teachers used the e-coaching website to prepare the LLL lessons $(n=12 ; 40 \%)$. No teacher used the website at the end of a lesson and very few used it the moment they encountered a difficult situation in the classroom $(n=5 ; 16.7 \%)$ or when they had a question $(n=2 ; 6.7 \%)$. 
The extent to which e-coaching was used by teachers was limited $(M=2.48 ; S D=0.83)$, with half of the teachers $(n=15)$ having visited only a few components of the website. Only $13.3 \%(n=4)$ of the teachers viewed all components of the website.

During website visit, teachers mostly read the tips ( $n=19 ; 63.3 \%)$, and role model stories $(n=18 ; 60 \%)$, watched videos $(n=14 ; 46.7 \%)$, and downloaded the student materials $(n=13 ; 43.3 \%)$. Less frequently visited were the FAQ $(n=8 ; 26.7 \%)$ and background information $(n=5 ; 16.7 \%)$. Only one teacher did the self-evaluation test, one asked a question, and one placed a reaction on the website.

SRH delivery difficulties that were viewed most often on the website were creating a safe atmosphere $(n=15 ; 50 \%)$, followed by dealing with homo-negative behavior in the classroom ( $n=11 ; 136.67 \%$ ) and guarding boundaries between teachers and students $(\mathrm{n}=8 ; 26.67 \%)$. Discussion in the classroom and provision of the lessons were also relatively popular $(\mathrm{n}=10 ; 33.33 \%)$.

Slightly more than half of the teachers $(n=22 ; 53.7 \%)$ intended to use e-coaching next school year when providing SRH lessons ( $M=3.34$; SD $=0.99) ; 7.3 \%(n=3)$ will certainly not use the website, $9.8 \%(n=4)$ probably not and the remaining $(n=12 ; 29.3 \%)$ were undecided (maybe/maybe not).

\section{Motives for teacher's (continued) use of e-coaching}

To understand teachers' motives to use or not use e-coaching, outcomes of both the survey and interviews were used.

In the interviews, teachers explained that they visited the website mainly to obtain student materials they could use in the classroom such as worksheets or facts and figures.

"I've given these lessons for 10 years and have developed my own routine. I enjoy bringing in new things once in a while. So, I looked on the website to see if there were any new things for me, to use in my lessons. That was not the case." (Teacher, 41 years, male).

In the interviews, the teachers mentioned they were triggered to visit the website after being advised during the teacher training from the MHS, or after receiving a reminder e-mail to use the website. The teacher manual, however, was seldom a trigger to go to the website. The trigger of the MHS training for using the website was supported 
by the survey, in which teachers who had received an MHS training were found to use e-coaching more frequently $(r=.47 ; p=.02$; Regression weight $=0.57$ (.24); $95 \%$ C.I. for $\mathrm{B}=0.08,1.06)$.

The survey revealed that teachers who visited the e-coaching website had a significantly more positive attitude towards reflecting about their own expertise $(M=6.24$; $\mathrm{SD}=.69 ; \mathrm{t}=3.03 ; \mathrm{p}=.01$ ) at baseline as compared to teachers not visiting the e-coach, within the intervention group $(\mathrm{M}=5.40 ; \mathrm{SD}=.91)$. They also perceived a significantly bigger need for support in providing $S R H$ lessons $(M=3.50 ; S D=.84 ; t=3.40 ; p=.00$ vs $M=2.30$; $\mathrm{SD}=1.25)$ and felt fairly less experienced in teaching $\mathrm{SRH}(\mathrm{M}=4.18 ; \mathrm{SD}=1.81 ; \mathrm{t}=-2.31$; $p=.03$ vs $M=5.60 ; S D=1.17$ ) at baseline as compared to teachers not visiting the e-coach. No other significant differences in demographics were found (ts $\leq 3.40$; ps $\geq .18 ; x=.30 ; p=.58)$.

As for future use, teachers were more likely to continue using e-coaching the following school year if they perceived the website as instrumental $(r=.38 ; p=.04)$ and usable $(r=.49 ; p=.01)$, if they also intended to use LLL next school year $(r=.55 ; p=.00)$ and had a higher subjective norm $(r=.48 ; p=.00)$, instrumentality $(r=.39 ; p=.01)$ and outcome expectations $(r=.42 ; p=.01)$ with regard to LLL as measured at post-test. None of these factors, however, significantly predicted intention to continue using e-coaching (ts $\leq 2.06$; ps $\geq .06$ ). The interviews revealed that teachers intended to continue using e-coaching $(\mathrm{N}=6)$ because they found the website supportive or wanted to use it to recap knowledge or prepare lessons or to get new updates.

\section{Motives for teachers' non-use of e-coaching}

The interviews revealed motives for teachers' non-use of e-coaching. According to the interviews, teachers stated the reasons for not filling out the self-reflection tool, namely that it was too time consuming, and inaccessible due to technical problems for the largest part of the pilot study.

The reasons why e-coaching in general was not used at all or not used more than once by the teachers can be categorized into three major reasons, namely i) low perceived personal relevance of e-coaching, ii) lack of time and iii) introduction of two innovations at once. 


\section{i) Low perceived personal relevance}

Teachers did not visit the e-coaching website at all or visited the website only once and then discontinued use mainly due to the low perceived personal relevance. This low personal relevance is explained in the interviews by teachers' unmet expectations by the website, extensive experience teaching SRH and lack of a perceived need for coaching.

\section{Unmet expectations}

The content of the website did not fit the expectation of teachers for finding numerous student materials they could use to assist them in providing these lessons, such as worksheets and alternative working methods and so they did not find e-coaching personally relevant and consequently no longer visited the website.

"These kinds of videos are nice to see. I may have clicked on them earlier and taken the time to watch the videos because hearing information in this way is new for me. But the thing is, I cannot do anything with this information in my lessons and that is what I was searching for." (Teacher, 56 years, female).

Furthermore, some teachers did not expect to be helped by a website; Teachers did not believe that the website or any website for that matter, could help them solve their problems, thereby not perceiving e-coaching as personally relevant.

"The questions I have cannot easily be solved. It also has to do with the school system, group processes and you can't solve it all with a website." (Teacher, 41 years, male).

\section{Extensive experience teaching SRH}

Teachers experienced in teaching SRH did not see the personal relevance of the website and discontinued use because it did not add anything new to what they already knew. 'I already know this', 'useful but not for me' were common expressions. These teachers had already implemented the suggestions presented or had already figured out a way of dealing with the difficulty.

"Yeah, you know, great that all this information is on the website, because it's all correct. But it's just a confirmation of what you already know" (Teacher, 43 years, male). 
They found the website better suited to and more relevant for less experienced teachers, for example, those who cannot guard boundaries or who find it difficult to deal with this subject and discuss it with students.

"I think it's very important for new colleagues, for those who have limited experience with the subject. I had less of a need to read everything. Firstly, it costs too much time and I don't have a need for it because I myself have so much experience. Teachers can say what they want but I have my own issues and I know how my students react. I know how I teach and how students react to that, so I didn't have a need to read all those stories. I did discuss the website with a beginner teacher and she viewed the website and I think she benefited from it" (Teacher, 55 years, male).

"I can imagine that the website is useful for teachers who are using LLL for the first time. Those who have never taught SRH, I think they can gain a lot of support from this website but for me, it's already known." (Teacher, 43 years, male).

Certain less experienced teachers, however, did not find the information on the website concrete and practical enough, due to their lack of experience, rendering the website less personally relevant for them as well.

"Young teachers have support needs but have very little experience and do not know yet what they would like to or should know. Older teachers have so much experience they think they have no need for support whatsoever." (Teacher, 55 years, male).

\section{Lack of perceived need for coaching}

During the interviews, teachers expressed a limited need for support in implementing LLL. They mostly believed that the delivery of their lessons was good enough as it was, thereby finding the website less personally relevant and not visiting the website at all.

"I think it's personal. If you are a teacher who wants to develop himself and are searching for a better way of teaching, then you will use this website. Otherwise you just teach the way you always have. If you would look in our school, you would find teachers who teach these lessons the way they do. Management can tell them they have to do it differently but they won't. Simply because the way they are doing it now works well, well enough." (Teacher, 50 years, male).

This limited need for coaching appeared to be related to various factors identified in the interviews, namely teachers already having years of experience teaching $\mathrm{SRH}$, receiving sufficient support from colleagues or preferring to consult a colleague with a question 
or problem instead of the e-caching website, and minimal difficulties encountered during implementation of LLL as the program was easy to work with, and the teacher manual gave sufficient guidance.

"I wouldn't look on the website. I'd be more likely to consult my colleagues who also teach that class of students and ask them what they did. How do they handle it and can we do something about this together? No, I wouldn't go looking for something like this on a website. I did enjoy reading certain things on the website but if I personally have a problem I won't look on the website." (Teacher, 43 years, male).

\section{ii) Lack of time}

A shortage of time is another reason influencing (lack of) website visit.

"Look, the website is of course a wonderful medium, to foresee people of information and advice. But what I notice in my daily practice is that you don't have the time to explore it further" (Teacher, 55 years, male).

Some teachers stated they did not have time or did not make time to explore the website. Not taking the time to visit the website could have contributed to teachers not being aware of the content of the website and to the lack of personal relevance. Numerous teachers remarked at the end of the interview that they perceived the website differently after having seen more of it.

"Well, now that I have viewed the website more thoroughly, I could definitely use some of the things, also to discuss with other colleagues next school year. What can we gain from this website? What did we learn from it? What extra information can be found on it? For example, the self-evaluation quiz, I think it's something we could do together with our colleagues. And then see, ok, are we on the same track, where are we missing information?" (Teacher, 27 years, female)

\section{iii) Two innovations at once}

The introduction of two innovations at once, the revised LLL program and the e-coaching website, seems to be another important reason why teachers did not use e-coaching. Teachers mentioned they first wanted to try the new LLL program and master it before using the website:

"I knew I could find information on the website but I preferred to first see how the LLL lessons go and if I encountered a difficulty, I would have a look at the website to see if someone had suggestions for it." (Teacher, 50 years, male). 
The frequency of use of the website may increase as teachers gain more experience with LLL:

"As you get more familiar with the LLL lessons, the next school year, you will feel a need to do more with the lessons and then such a website becomes interesting." (Teacher, 42 years, female).

\section{DISCUSSION}

A web-based e-coach, (lesgevenindeliefde.nl-teachinglove.nl) was developed to support teachers in implementing the Dutch school-based sexual health program, Long Live Love (LLL), with completeness and fidelity. An effect- and a process evaluation were conducted to evaluate the effectiveness of the e-coach in influencing teachers' implementation as well as to evaluate the teachers' use and appreciation of the e-coach. E-coach was not found to have an effect on teachers' implementation behavior (see Schutte et al., 2017, accepted for publication under review). The process evaluation reported in this paper showed that even though aspects of the e-coach were appreciated and perceived as usable and instrumental, the website was very limitedly visited, was perceived as less personally relevant and could in itself be improved.

\section{Use of e-coaching}

To have the desired effect, however, it is crucial that an intervention be used and used accordingly (Bartholemew et al., 2016). E-coaching was not used or insufficiently used. This suboptimal use of e-coaching is likely the reason why it did not have any significant effects on (determinants of) implementation behavior of teachers.

Factors that stimulated use and continued use of e-coaching were a positive attitude towards LLL, a positive evaluation of the e-coaching website as instrumental and usable, expecting to find student materials on the website and receiving an MHS training. Lack or non-use of the website, according to teachers, was due to a lack of time, the introduction of two innovations simultaneously and limited perceived personal relevance of e-coaching due to unmet expectations of finding student material to use in the classroom- 'I didn't find what I was looking for', extensive experience teaching $\mathrm{SRH}$, thereby not finding new information on the website- 'I already know this' and no perceived need for coaching- 'useful, but not for me'. 
E-coach developers had taken lack of time of teachers into consideration as a potential barrier to use of e-coaching, which was mentioned in the needs assessment phase, by developing a website that was easy to use, flexible and accessible (Schutte, 2016). These aspects of e-coaching were appreciated by teachers, yet time remains a universal barrier in the implementation of school-based health education programs (Ahtola, Haataja, Karna, Poskiparta, \& Salmivalli, 2013; Bessems et al., 2014; Buston et al., 2002; van Lieshout, Mevissen, de Waal, \& Kok, 2016; Wind et al., 2008).

With the introduction of two innovations at once, LLL and e-coach, teachers did not use e-coaching at all or did so minimally. The process of accepting an innovation, such as e-coaching, takes time, as described in Rogers' diffusion curve (Rogers 2003). The innovation will most likely be adopted by innovators first, followed by the early majority and eventually the laggards. Pijpers et al., (2002) claim that online innovations first need to be accepted in order to be used broadly and effectively.

Low personal relevance of e-coach was fundamental to limited use of the website. Personal relevance has been found to be related to use of internet-delivered interventions (Crutzen, Ruiter, \& de Vries, 2014). The lack of perceived personal relevance of e-coaching was, on the one hand, related to teachers' unmet expectation of finding student materials on the website that they could use to assist them in providing these lessons, leading to discontinued website use. A large study in the Netherlands found that teachers in secondary schools either consult colleagues in their school for information or use the internet mainly to find information, prepare their lessons, send e-mails to students or give homework assignments and thus less for professional development (Giling \& Laan, 2005). Especially experienced teachers did not perceive the website as personally relevant because the information on the website did not add anything new to what they already knew or had already implemented. They found the website better suited to less experienced teachers and thus discontinued use. Teachers who lacked a perceived need for coaching did not visit the website at all because they did not perceive it as personally relevant. Teachers expressed a minimal need for coaching because they were convinced of their teaching methods-'I'm teaching well'- and they did not perceive their implementation behavior of selectively using program components, adjusting the program with their own additions and delivering the program incompletely as problematic. Especially teachers who were experienced in teaching SRH, who perceived LLL as easy to use, who did not encounter difficulties or who already received support from colleagues felt no need for coaching. The absence of the need or desire of teachers to be coached was fundamental to the limited use and lack of effect of e-coaching on implementation behavior. 


\section{Discrepancy in perceived need for coaching}

This lack of perceived need for coaching was already established when developing e-coaching and reconfirmed in this process evaluation. The problem was therefore, that despite their implementation behavior being suboptimal, according to program developers, teachers themselves did not perceive their teaching behavior as problematic and felt resistance to coaching or behavioral change, even after exposure to e-coaching.

Based on the findings of this process evaluation, we realized in hindsight that the discrepancy that exists between teachers and e-coach developers on the perceived need for coaching or behavioral change appears to be related to a different interpretation regarding quality of teaching. For teachers, 'correct program delivery' and thus good teaching is related to having teaching material for students and sufficient didactic skills to deliver lessons. This was reflected in how teachers used the e-coaching website, as reported in this study; namely searching for student materials, discontinuing website use if they did not find the materials they were looking for and focusing on difficult situations mainly related to didactics, such as discussion in the classroom. For program developers, however, 'correct program delivery' is related to implementation with completeness and fidelity and dealing adequately with challenging situations that may arise in the classroom during program delivery in order to achieve program effectiveness. Teachers and program developers were thus approaching the problem from different reference frameworks and both were right from their own perspectives: Teachers felt they were teaching well because they were considering their teaching behavior from their reference framework of didactics, which is their profession and expertise, and thus did not perceive a need for teaching support in that area, rendering e-coaching less personally relevant. Teachers were not thinking in terms of completeness and fidelity, as program developers were. Teachers therefore appeared to have limited awareness of the implementation problem and did not seem to understand the importance of completeness and fidelity in delivery of SRH lessons. Without recognizing the importance of completeness and fidelity in providing SRH lessons, teachers will not feel a need for coaching in that area. Lack of awareness of the importance of completeness and fidelity was found to be related to incomplete program implementation of the online schoolbased sex education program LLL+ for older high school students (van Lieshout et al., 2016). This could explain why perceived need for coaching remained unchanged, even after exposure to e-coaching. 


\section{E-coaching intervention}

E-coaching seemed unable to change teachers' perceived need for coaching possibly because the importance of completeness and fidelity were not sufficiently or explicitly enough addressed in e-coaching itself and in the promotion activities surrounding the e-coach. Understanding the importance of completeness and fidelity was an essential first step to provide teachers with a motive to visit the website, prior to coaching. Previous research has shown that teachers' incomprehension of the theoretical basis for behavior change negatively influences implementation (van Lieshout et al., 2016; Buston et al., 2002, Ruiter, Kessels, Peters, \& Kok, 2014). Coaching can only commence when teachers develop awareness of their needs and desires to improve their classroom performance (Parsloe \& Leedham, 2009).

Critical self-reflection was an essential next step to create awareness of own teaching behavior and a need to be coached or for behavioral change. The most important tool for this was the self-reflection tool but it was inaccessible for most of the pilot study due to technical problems, and combined with the limited use of the website, selfreflections was not realized.

The lack of effect of e-coaching could also partially be attributed to the development of the e-coaching intervention itself. The development was guided by what teachers mentioned they needed support in, which in hindsight we realize, was mainly didactic support; teachers referred to 'teaching $\mathrm{SRH}^{\prime}$ and 'need for coaching' from their own reference framework of didactics. As a result, e-coaching, being tailored to the requests of teachers, focused predominantly on dealing with difficult situations, which are the preconditions required to deliver SRH lessons with completeness and fidelity. Although e-coach was ultimately aimed at promoting completeness and fidelity of implementation of LLL, it did so largely by focusing on removing the barriers for teaching SRH in general. This is only one aspect of achieving completeness and fidelity. It may have insufficiently addressed direct means of achieving completeness and fidelity of LLL specifically, in the form of practical tools and guidelines. Creating prerequired conditions for teaching SRH generally and ability to deliver LLL lessons specifically in its entirety and as prescribed are both essential for maximizing likelihood of achieving completeness and fidelity of LLL. Although the e-coaching intervention contains elements required to create the prerequired conditions for completeness and fidelity, in its current form, the e-coach may not have been ideal due to perhaps containing insufficient content to directly influence completeness and fidelity of LLL specifically. This could explain why the effect on these outcome measures was not found. 


\section{Sample bias}

The teachers who agreed to participate in the interviews and the study may have been a biased sample of motivated, experienced teachers who were already capable of delivering LLL successfully, thereby feeling no need for e-coaching.

\section{Implications}

As long as teachers perceive their teaching as non-problematic, they will not feel a need for change and coaching. Coaching can only commence when teachers develop awareness of their needs and desires to improve their classroom performance (Parsloe \& Leedham, 2009). Having realized that we were approaching the problem from different reference frameworks, teachers and program developers need to create a mutual understanding of what 'correct program delivery' is. More research needs to be conducted to explore this discrepancy in 'correct program delivery'. Teachers should be asked whether they perceive completeness and fidelity as important and how they can be supported in doing so. These insights can then be incorporated into the implementation strategy to improve implementation quality.

While respecting the professionalism of teachers and acknowledging their reference framework of good teaching, which involves didactics, it seems important, first and foremost, to ensure teachers see the personal relevance of coaching or behavioral change. This is important for the use of e-coaching. Website use was found to be related to factors associated with the visitor (such as their motivation to be healthy) (Crutzen, de Nooijer, Candel, \& de Vries, 2008; Riet, Crutzen, \& Vries, 2010) as well as the intervention website (such as offering tailored information) (Brouwer et al., 2011; Crutzen et al., 2011; Nijland, Van Gemert-Pijnen, Kelders, Brandenburg, \& Seydel, 2011). The e-coaching intervention itself thus also needs to be improved. Not only should it include information emphasizing the importance of completeness and fidelity generally but would also need to include tools to enhance fidelity and completeness of LLL specifically. For example, a video of other teachers explaining how to use the teacher manual and deliver all six lessons within a particular period of time. Or guidelines in the teacher manual to deliver principal components of the LLL program to preserve effectiveness. The self-reflection tool, which is essential in creating awareness of own teaching behavior, will need to be optimized into a shorter, more interactive quiz, for instance. Exploring other means of promoting self-reflection is also possible, for example, during teacher trainings.

Increasing the personal relevance of the website is necessary to get teachers to the website and keep teachers on the website and prevent discontinued use. Increasing personal relevance of a website was found to be a promising strategy to increase use of internet-delivered interventions (Crutzen et al., 2014). To increase e-coaching's' personal 
relevance (and create a need for coaching or behavioral change) the importance of completeness and fidelity for program effectiveness should be highlighted and specified as a component of good teaching of SRH. This will provide a motive to visit the website. The broader implementation strategy should then be utilized to bridge the intentionbehavior gap and promote actual website use. Implementation promoting activities should be deployed such as distributing information leaflets and supporting the MHS in the provision of teacher trainings, for example. Teachers can subsequently be coached into self-reflection, dealing adequately with difficult situation and implementing LLL with completeness and fidelity, via e-coaching. In this way, support of teacher delivery of LLL and SRH generally will encompass not only completeness and fidelity, but also assistance in creating the prerequired conditions necessary for providing these lessons.

Implementation of implementation enhancing interventions, such as e-coaching is thus not guaranteed and requires planning. Voogt et al., (2016) highlighted the limited use of IT by teachers in Dutch school-based settings. Limited use of online interventions in the school-setting, however, does not automatically imply that web-based coaching in itself is an ineffective strategy to support delivery of health promoting programs in schools. Digital technologies are being increasingly used in the education system, bringing exciting opportunities for innovative ways of teaching and learning (Ertmer \& OttenreitLeftwich, 2010). Exploring the educational potential of these digital technologies and supporting schools in making use of them remains important (Ertmer \& OttenreitLeftwich, 2010).

Even if e-coaching would be used optimally, organisational constraints to implementation, such as time shortage, will always be present (Ahtola et al., 2013; Bessems et al., 2011; Buston et al., 2002; Wind et al., 2008). Although completeness and fidelity are important, the fact that almost no school-based program seems to be delivered with completeness and fidelity brings into question whether LLL or other school-based (sex) education programs can realistically be expected to be delivered with absolute completeness and fidelity as program developers require (Durlak \& DuPre, 2008). Adaptations, such as additions or omissions, often occur and are sometimes inevitable to accommodate local situations. Keeping these adaptations in mind and considering that absolute completeness and fidelity cannot realistically be expected, teachers should be supported in delivering programs with as much completeness and fidelity as possible, with guidance in delivering principle program components to ensure program effectiveness is preserved (Han \& Weis, 2005). This requires two things: (1) the program must be developed and structured with sufficient flexibility such that it can be adapted to changing circumstances and (2) teachers must understand the program well enough and be skilled and guided enough so that they are able to modify it without 
sacrificing the core principles and central intervention techniques (Dusenbury et al., 2003). An example of an SRH program that included choice options and guidance to enhance completeness and fidelity is the Long Live Love + (LLL+) program for older high school students (van Lieshout et al., 2016). Environmental factors that form barriers to teacher implementation, such as school policy, culture, support and socio-political context, form the prerequisites for successful implementation and need nonetheless to be taken into consideration when thinking of promoting implementation.

\section{CONCLUSION}

This process evaluation revealed that suboptimal use of e-coaching is likely the reason why it did not have an impact on (the determinants of) teacher's implementation behavior, along with perceived short-comings of the e-coaching website itself. While it remains important to respect the professionalism of teachers, it is essential to ensure that teachers and program developers have a mutual understanding of'correct program delivery' by highlighting the importance of completeness and fidelity as a component of good teaching. This is an essential first step to behavioral change. We understand that supporting effective delivery of SRH programs encompasses not only promoting completeness and fidelity but also requires a well-developed program and support in creating the prerequired conditions necessary for delivering SRH lessons, such as creating a safe atmosphere to discuss sexuality. Implementation of implementation enhancing interventions, such as e-coaching is not guaranteed and needs to be planned for. As program developers, we need to ask ourselves how realistic it is to expect absolute completeness and fidelity of program delivery in the school setting. We should focus on supporting teachers in delivering programs with the highest level of completeness and fidelity possible, yet guide them in delivering the components of the intervention that are crucial for effectiveness.

\section{ABBREVIATIONS}

IM: Intervention Mapping

LLL: Long Live Love

MHS: Municipal Health Services

SRH: Sexual Reproductive Health 

CHAPTER 7

General discussion 



\section{GENERAL DISCUSSION}

The success of health promotion programs is not only dependent on the quality and content of the program but also on the quality and extent of its implementation (Durlak \& Dupre, 2008). Implementation is considered a process consisting of different stages, namely dissemination (i.e. awareness), adoption (i.e. form an intention, uptake, initiate, commit, accept a program), implementation (i.e. initial use), and continuation (i.e. continued use) (Rogers, 2003). The implementation process thus refers to the sum total of each of these stages. Within the adoption stage, different categories of adopters are identified, based on the speed at which they tend to accept an innovation. These are innovators, early adopters, early majority, late majority, and laggards. (Rogers, 1995). The implementation stage has been defined by two dimensions, essential for increased likelihood of program effectiveness: completeness (i.e. the extent of the program being delivered) and fidelity (i.e. the degree to which the program has been implemented as intended by the developers and as prescribed in the original protocol) (Durlak, 1998).

The main objective of the work presented in this dissertation was to systematically develop and evaluate a national implementation strategy for the optimal adoption, implementation and continuation of the revised secondary school-based sex education program, 'Long Live Love' - 'Lang Leve de Liefde' (LLL). The implementation strategy was based on theory and evidence and was developed in close collaboration with teachers and Municipal Health Service (MHS) professionals in the Netherlands. It included interpersonal contact, multimedia, and online approaches, to address each stage of the implementation process. The implementation strategy was aimed at secondary school teachers who (are willing to) teach sex education. MHS professionals are intermediaries who function as environmental agents, influencing the teachers' adoption and implementation of sex education. They support the implementation process of LLL by delivering the implementation strategy; namely by conducting adoption- implementation- and continuation promoting activities to support teachers in this process and thus to optimize the implementation of LLL. See Figure 1 for an overview of the actors and actions involved in the development and delivery of the implementation strategy. 


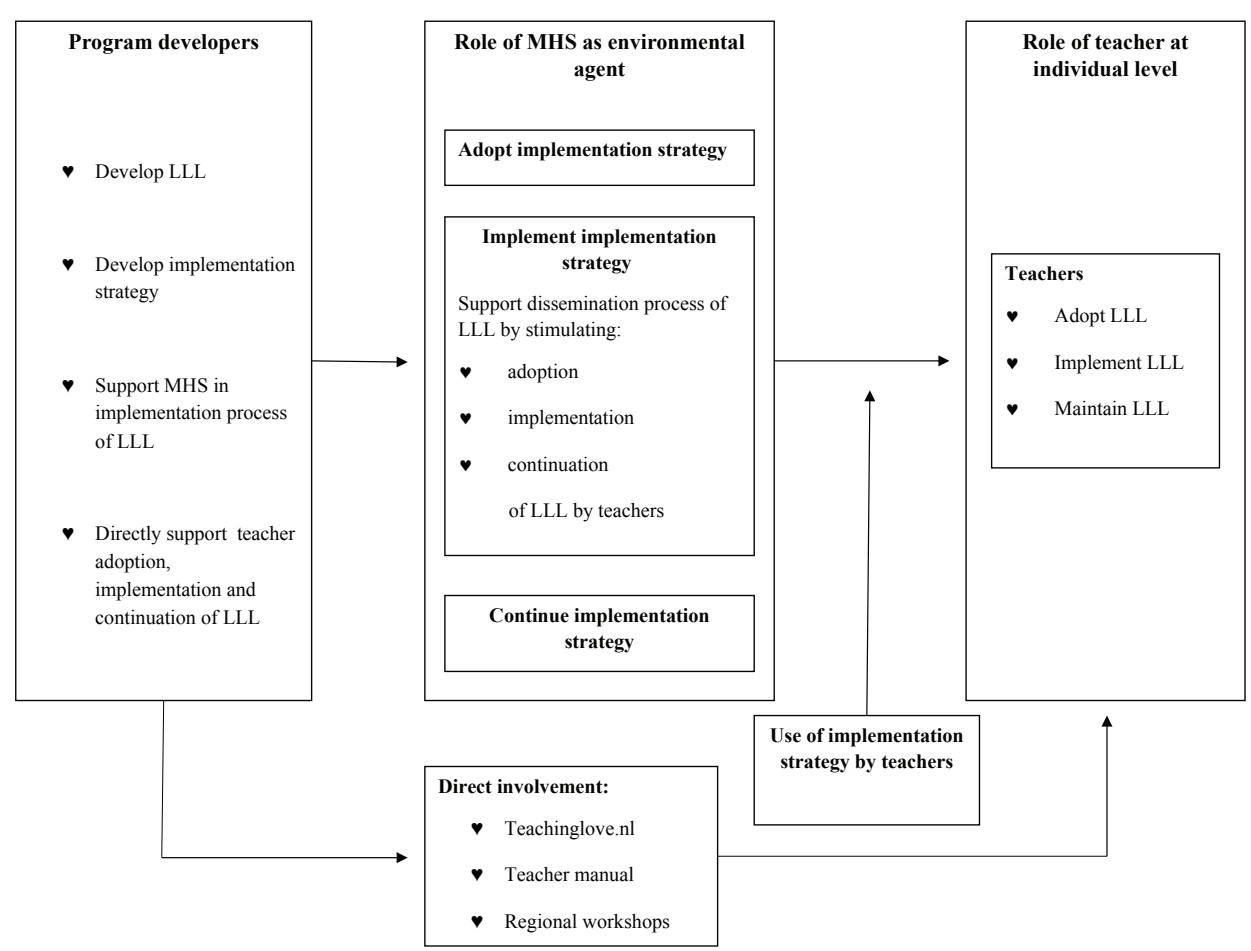

Figure 1. Actors and actions in the implementation strategy.

The MHS was supported by the program developers in their LLL implementationpromotion tasks by means of training and follow-up support and by the provision of scripts on how to prepare, implement and continue the implementation strategy, including supportive instruments such as model recruitment letters, information leaflets and protocols. This simplified their work and ensured a uniform approach.

Teachers were stimulated, predominantly via the MHS but also directly via program developers, to adopt, implement and continue use of LLL to ultimately improve the sexual health of students. Tools such as an information leaflet, teacher training, previews of the LLL material, including a trailer of the DVD films, a teacher manual, regional comeback meetings and evaluation forms were used.

Different tools were therefore developed as part of the broader implementation strategy to promote each stage of the implementation process of LLL. These tools were tailored to determinants of that stage. Within the implementation stage, completeness and fidelity are especially important for program effectiveness. The web-based coaching intervention, 'lesgevenindeliefde.nl' or 'teachinglove.nl' was developed as a tool, 
within the broader implementation strategy, to promote this stage. This e-coaching intervention was aimed at supporting teachers directly during implementation of LLL in delivering LLL with completeness and fidelity and in dealing with the most common difficulties encountered when delivering Sexual and Reproductive Health lessons (SRH) generally and LLL lessons specifically. Being the most innovative component of the broader implementation strategy, e-coaching is the focus of this dissertation. See Table 1 for a more detailed description of the broader implementation strategy, including the e-coaching component. 
Table 1. Broader implementation strategy of LLL: MHS and teachers.

\begin{tabular}{|c|c|}
\hline MHS & \\
\hline $\begin{array}{l}\text { Behavioral outcome (BO): Adopt implementation strateg } \\
\text { implementation process of LLL by teachers in schools }\end{array}$ & $y:$ MHS professionals decide to support the \\
\hline $\begin{array}{l}\text { Performance objective }(P O) \text { (activities): Decide to support } \\
\text { implementation process of LLL }\end{array}$ & $\begin{array}{l}\text { Tools in toolkit to help MHS support implementation process } \\
\text { of LLL }\end{array}$ \\
\hline $\begin{array}{l}\text { - MHS professionals accept LLL as a valuable program for } \\
\text { proving sex education in schools. } \\
\text { - MHS professionals decide to support the implementation } \\
\text { process of LLL by teachers in schools. } \\
\text { - MHS professionals follow a train-the-trainer workshop. } \\
\text { - MHS professionals review LLL curriculum. } \\
\text { - MHS professionals review teachinglove.nl website. } \\
\text { - MHS professionals review dissemination toolkit on USB } \\
\text { stick. }\end{array}$ & $\begin{array}{l}\text { - Facts \& figures report on sexual health of Dutch youth } \\
\text { - Protocol to recruit MHS } \\
\text { - Letter to inform MHS about LLL } \\
\text { - Information leaflet LLL } \\
\text { - LLL curriculum } \\
\text { - Introduction film LLL } \\
\text { - LLL DVD trailer } \\
\text { - Teachinglove.nl trailer } \\
\text { - Sample budget request at municipality } \\
\text { - Train-the-trainer workshop: quiz with information about } \\
\text { sexual health of Dutch youth, introducing LLL and } \\
\text { practice with curriculum, information about teachinglove. } \\
\text { nl, role of MHS in supporting implementation process of } \\
\text { LLL, review of tools available to support MHS in delivering } \\
\text { the implementation strategy, LLL curriculum, USB stick }\end{array}$ \\
\hline
\end{tabular}

BO: Deliver implementation strategy: MHS professionals support teachers in the adoption, implementation and continuation of LLL in schools, completely and as prescribed by program developers

PO (activities): Stimulate dissemination and adoption of LLL Tools in toolkit to assist MHS professionals to stimulate dissemination and adoption of LLL

- MHS professionals inform teachers about LLL using - Protocol for recruitment schools: Included: personal and multi-media channels. $\quad$ Information leaflet

- MHS professionals use the support tools in the toolkit. - Model letter for recruitment schools

- MHS professionals recruit schools to use LLL. - Model letter of agreement

- MHS professionals send information about LLL program . Price overview LLL material to schools.

- Overview of themes per LLL lesson

- MHS professionals persuade teachers to use LLL for sexual introduction film LLL education.

- LLL DVD trailer

- MHS professionals follow-up the decision of schools to - Teachinglove.nl (e-coaching) trailer use LLL. • Model PowerPoint for parent-evening

- MHS professionals provide further (needed) information - Digital PDF versions of LLL curriculum about LLL to interested teachers.

$P O$ (activities): Stimulate implementation of LLL

Tools in toolkit to assist MHS professionals to stimulate implementation of LLL

- MHS professionals prepare teachers in the use of LLL.

- Instruction book for teacher training

- MHS professionals explain the components, content and working methods of LLL to teachers.

- Model PowerPoint presentation of teacher training

- MHS professionals inform teachers about where they can

- Road map for navigating through teachinglove.nl find support for implementing LLL in the classroom.

(e-coaching)

MHS professionals inform teachers about the importance

- Evaluation form for teacher training of completeness and fidelity in implementing LLL for effectiveness. 
Table 1. (continued)

\begin{tabular}{|c|c|}
\hline \multicolumn{2}{|l|}{ MHS } \\
\hline PO (activities): Stimulate continuation of LLL & $\begin{array}{l}\text { Tools in toolkit to assist MHS professionals to stimulate } \\
\text { continuation of LLL }\end{array}$ \\
\hline $\begin{array}{l}\text { - MHS professionals stimulate teachers to continue using } \\
\text { LLL for sexual education every school year. } \\
\text { - MHS professionals maintain contact with school: keep } \\
\text { teachers updated about new changes made to the LLL } \\
\text { program. } \\
\text { - MHS professionals follow up with teachers after } \\
\text { completion of LLL. } \\
\text { - MHS professionals remind teachers to incorporate and } \\
\text { use LLL next school year. } \\
\text { - MHS professionals facilitate LLL teachers to share their } \\
\text { experiences with other teachers once a year. } \\
\text { - MHS professionals structurally offer their support services } \\
\text { to schools every school year. }\end{array}$ & $\begin{array}{l}\text { - Protocol for maintaining contact with schools } \\
\text { - Postcards LLL } \\
\text { - Excel document to monitor use of LLL } \\
\text { - Evaluation forms LLL lessons (mail or telephone) } \\
\text { - Referral of teachers to MHS for required support via the } \\
\text { longlivelove.nl platform }\end{array}$ \\
\hline
\end{tabular}

BO: Continue implementation strategy: MHS professionals continue to support the implementation process of LLL by teachers in schools

PO (activities): Continue supporting implementation process of LLL

Tools in toolkit to help MHS continue supporting implementation process of LLL

- MHS professionals incorporate the dissemination strategy in their MHS policy.

- Regional yearly comeback meetings

- MHS professionals create support in the MHS for LLL.

- Newsletter with updates

- MHS professionals receive budget from the provincial - Evaluation forms train-the-trainer workshop public health services to support the implementation - Input for adaptation of LLL process of LLL in the next 5 years.

- Implementation toolkit on USB stick

- MHS professionals receive updates about implementation of LLL and continued support from program developers.

\section{TEACHERS}

Behavioral outcome (BO): Dissemination: Teachers are aware of LLL for providing sex education to secondary school students.

Performance objective (PO) (activities): Aware of LLL for sex education

Dissemination strategy: Tools to stimulate awareness of LLL among teachers

- Teachers are open to new sexual education programs.

- Regional workshops

- Teachers actively seek information about sexual • Information leaflet education programs, including LLL.

- Newsletters

BO: Adoption: Teachers decide to use LLL for proving sex education to secondary school students.

PO (activities): Decide to use LLL

Adoption strategy: Tools for stimulating adoption of LLL by teachers

- Teachers agree that relational and sexual education is needed.

- Information leaflet

- Teachers review the LLL program material.

- Facts \& figures report on sexual health of Dutch youth

- Letter to inform parents about provision LLL lessons.

- Teachers decide to use LLL for providing sexual education.

- Teachers inform parents and colleagues about LLL.

- Teachers buy the LLL program.

- Teacher Training including: quiz on sexual health Dutch youth, introduction film LLL, LLL DVD trailer, teachinglove. $\mathrm{nl}$ trailer, information about LLL curriculum, certificate of participation 
Table 1. (continued)

\section{TEACHERS}

BO: Implementation: Teachers deliver LLL as sex education for secondary school students

PO (activities): Initial implementation: Teachers deliver LLL to students.

- Teachers plan time for giving LLL lessons.

- Teachers integrate LLL as part of the (biology) lessons.

- Teachers prepare themselves for the use of LLL.

- Teachers deliver the LLL program to students for sexual education.

PO (activities): Completeness: Teachers deliver at least $80 \%$ of LLL program content to students

- Teachers cover all six lessons of LLL.

- Teachers use all program materials of LLL in each lesson (DVD, magazine, teacher manual).

- Teachers cover the most important components of each lesson, as indicated in the teacher manual.

PO (activities): Fidelity: Teachers deliver LLL to students according to the guidelines in the teacher manual.

- Teachers read the teacher manual as preparation for each lesson.

- Teachers create a safe and secure atmosphere in the classroom during all LLL lessons.

- Teachers teach all themes in LLL without shame or taboos.

- Teachers handle all difficult situations that arise in the classroom during LLL adequately. These situations are dealing with homo-negativity in the classroom, dealing with personal questions, diversity in culture and experience, differences between boys and girls and individual problems.

- Teachers deliver each LLL lesson to students according to the teacher manual.

BO: Continuation: Teachers continue to deliver LLL to secondary school students every school year for sex education

PO (activities): Teachers continue to deliver LLL for at least 3 years

- Teachers structurally integrate curriculum.

- Teachers plan time for LLL lessons for each following school year.

- Teachers buy additional LLL4 material on time.

- Teachers deliver LLL every school year to secondary school students for as long as they are teaching the relevant subject in the school.

Implementation strategy: Tools for stimulating

implementation of LLL by teachers

- Teacher training: practice with LLL material, inventory of most common difficult situations, practice with case studies and discuss role model stories, watch videos

- Teachinglove.nl (e-coaching)

- Teacher manual

Tools in implementation strategy to stimulate completeness

- Teacher training

- Teachinglove.nl (e-coaching)

- Teacher manual

Tools in implementation strategy to stimulate fidelity

- Teacher training

- Teachinglove.nl (e-coaching)

- Teacher manual 
The quality and content of LLL as well as a successful implementation of LLL should ultimately lead to young people being better prepared, motivated and able to practice safe sex and have mutual, pleasant relationships (Schaalma et al 1996; Durlak \& Dupre, 2008). Implementation bridges the gap between developing the intervention and reaching the target group. Implementation is nevertheless often an aspect which is overlooked, insufficiently considered, not planned for or poorly conducted, regularly leading to program failure (Green \& Kreuter, 2005). By focusing on implementation, this dissertation highlights the importance of implementation to reach the target group and have an impact on public health. It exemplifies the complexity of all the factors and actors that influence this dynamic process, and the effort required to ensure successful implementation.

Each chapter of this dissertation describes the steps taken to systematically develop an implementation strategy, using a participatory development approach involving teachers (end users) and MHS professionals to reach secondary school students with the revised LLL and have an impact on their relational and sexual health. The Intervention Mapping (IM) protocol guided the systematic and planned development of the implementation strategy, targeting teachers. This IM protocol stimulates a baseline (or needs) assessment of the factors associated with the implementation process and an exploration of the environmental conditions that may influence implementation success, in this case the needs and capacity of the MHS as environmental agent, who forms the link between the program developers and the schools.

A needs assessment was conducted in three studies presented in this dissertation: a qualitative study focusing on teachers' implementation process of the previous LLL and its' related determinants (see chapter 2), a qualitative and quantitative study among the MHS professionals as environmental agent focusing on their current and future supportive role in the implementation process of LLL (see chapter 3), and a qualitative study among teachers prior to e-coach development focusing on their implementation behavior and their (perceived) need and preferences for coaching in delivering sexual and reproductive health (SRH) programs (see chapter 4). Starting with an analysis of factors associated with teachers' adoption, implementation and continuation of the previous LLL program (see chapter 2), we realized that implementation was not optimal; teachers implemented $64.1 \%$ of the learning activities in the previous LLL and modified their lessons. With the outdated LLL program now being revised, a strategy was needed to enhance completeness and fidelity of LLL. Adoption and continuation of the revised $L L L$ also needed to be stimulated. The different determinants influencing each implementation stage were therefore taken into account. Next, insights were obtained by means of qualitative and quantitative research, from the principal environmental 
agents in the implementation process of LLL, namely MHS professionals. The capacity and motivation of the MHS professionals to support teachers in the implementation process of LLL was investigated (chapter 3). With the capacity of the MHS being restricted at the time the revised LLL program would be ready for implementation due to the economic crisis, along with their lack of didactic skills and expertise to be appropriate role models for teachers in teaching skills for adequate implementation, another form of support was needed to compensate for the limitations of the MHS and support teachers in the implementation of LLL with completeness and fidelity. Additionally, instead of providing program materials (including a teacher manual) and one-time pre-implementation training via the MHS, ongoing support to complement the existing implementation strategy was required once the transition was made to real-life implementation of LLL by teachers. Lastly, the needs and barriers encountered when delivering sex education were identified among teachers themselves in the needs assessment stage for e-coaching development to support them with completeness and fidelity (chapter 4). Outcomes of research in all three studies formed the foundation for developing each component of the broader implementation strategy, including e-coaching. It gave insight in the possibilities and barriers to implementation, the factors that needed to be addressed in the implementation strategy and that needed to be considered in developing the revised LLL program.

A broader implementation strategy was developed for LLL, addressing each stage of the implementation process, using the IM protocol, including the results of the abovementioned studies. This was attained by formulating specific goals to be achieved with the implementation strategy- step 2 of IM. Insights from the Diffusion of Innovation Theory (Rogers, 1995), the Theory of Planned Behavior (Ajzen, 1991) and the Social Cognitive Theory (Bandura 1986) were additionally used to identify the different determinants for each of the stages in the implementation process. In step 3 of IM, theoretical methods addressing these determinants were then selected and translated into practical applications for the implementation strategy development. A coherent implementation program was then designed for LLL in step 4, to ensure successful implementation of LLL, largely by mobilizing the MHS and providing direct support via program developers. An accompanying plan to ensure successful implementation of the implementation strategy followed in step 5, for example, by training MHS professionals and integrating e-coaching in the teacher manual and teacher trainings. The implementation strategy complemented the different working approaches used by the MHS, namely the 'Health Promoting School' (Gezonde School Methode) approach, in which the school, together with the MHS, prioritize a health topic and incorporate it into the school policy (Boot, van Assema, Hesdahl, Leurs, \& de Vries, 2010) versus the supply oriented approach in which the MHS actively approaches schools to promote 
and stimulate use of particular health promotion programs. To ensure effective dissemination of LLL in the school setting, we made use of existing networks and used a variety of formal and informal media and communication channels, which can enhance the durability of an innovation and ensure its long-term continued use (Oldenburg \& Parcel, 2002). The implementation strategy was also made relevant for experienced and less experienced teachers and took the different adopter categories into consideration, from innovators to laggards. Mass media as well as interpersonal contact was used to encourage adoption of LLL among the less innovative teachers (Wiefferink et al., 2002). Continuation was stimulated by interpersonal contact and implementation was enhanced by interpersonal and online approaches.

The focus of this dissertation is mostly on the development and evaluation of the most innovative component of this broader implementation strategy, namely the webbased coaching program for supporting teachers in implementation of LLL. Teachers, being key players in the implementation of school-based sexual health education programs, require support in every stage of the implementation process (Forman et al., 2009). Support is, however, often limited to enhancing program adoption, with less attention for stimulating completeness and fidelity of program delivery, despite this being crucial to program effectiveness (Bessems et al., 2014; Domitrovich \& Greenberg, 2000; Fagan \& Mihalic, 2003; Durlak, 1998). The results presented in chapter 2 showed that the completeness and fidelity of the previous LLL by teachers was not optimal and needed to be enhanced for the revised LLL. Although teacher implementation of LLL was previously supported by the MHS, the study presented in chapter 3 showed that, due to economic cutbacks at the time of data collection for the needs assessment, the supportive role of the Dutch MHS for the revised version of LLL was going to be limited to stimulating the dissemination and adoption of LLL and preparing teachers for initial implementation through training. In addition to lacking the capacity to provide intensive, long-term support, the MHS professionals appeared to lack the didactic expertise and skills to be appropriate role models for teachers in teaching skills for adequate implementation. Moreover, although pre-implementation training often equips teachers with skills for correct implementation, it is not enough (Dusenbury et al., 2003). It remains important to provide teachers with ongoing support and consultation during actual program delivery to ensure the quality of implementation (Schutte et al., 2014; Kramer at al., 2000; Rohrbach et al., 1993; Durlak, 1998). Teachers therefore needed another form of support during implementation to compensate for the limitations of the MHS and to provide ongoing support during implementation to enhance completeness and fidelity. Chapter 4 describes the systematic development of the e-coaching website, using IM, to support teacher implementation of LLL. The effectand process evaluation of e-coaching are described in chapters 5 and 6 . 


\section{Main findings}

The main findings per chapter in this dissertation will be described below.

\section{Factors promoting and impeding the implementation process of Long Live Love}

Chapter 2 describes the findings of a study among teachers $(\mathrm{N}=130)$ on factors associated with each stage of the implementation process in the school setting regarding the previous LLL sex education program. The results highlighted that implementation of LLL was not optimal and could be improved: approximately one-third of the LLL activities were not implemented and among the remaining two-thirds of the activities that were implemented, teachers generally did so with limited integrity; lessons were (slightly) modified. This finding is in line with several other studies also showing frequent modification of program components during implementation and teachers not always implementing programs according to specific guidelines (Sy \& Glanz, 2008; Durlak \& DuPre, 2008; Dusenbury et al., 2003). Our study showed that fidelity was especially hindered when teachers had more years of experience with LLL. Years of experience with a program may lead to reinvention of it by the user due to increased familiarity with the program and to accommodate the changing circumstances in schools and diversity in classroom composition in time (Berman \& McLaughlin; Ringwalt et al., 2003; Rogers, 2003).

Additionally, each stage of the implementation process was found to be influenced by different factors: teacher curriculum-related beliefs were primarily associated with adoption, but also with implementation and continuation. Additionally, implementation completeness and fidelity and continued use of LLL were specifically enhanced by contextual factors, namely teacher training and interactive context variables - school policy, governing body support, and student response. This is in line with several studies who found adoption to be predominantly related to individual level factors (Paulussen et al., 1995; Hoekstra et al., 2009) whereas implementation and continuation are also influenced by external factors, namely information source variables and the interactive context (Foreman et al., 2009; Payne, 2009; Payne et al., 2006; Paulussen, 1994). Teacher training as an information source variable has shown to not only be important to enhance implementation quality of LLL but school-based interventions in general (Wiefferink et al., 2005). The factors identified in this study were taken into consideration when developing the revised LLL program, in anticipation of implementation. They also provided insight into the factors that needed to be addressed in the implementation strategy. 


\section{Current and future role of the MHS - barriers and facilitating factors}

The study presented in chapter 3 provided insight in the barriers and facilitating factors influencing the (future) supportive role of the MHS in the implementation process of LLL. These insights were gathered by means of surveys $(N=26)$ and interviews $(N=19)$. In the Netherlands, the MHS is the external party responsible for stimulating health promotion activities in schools and plays an important role in the dissemination of health promoting programs and supporting the adoption and implementation of such programs by teachers in schools. The quality with which the MHS supports implementation promoting activities of LLL influences the subsequent implementation quality of LLL by teachers in schools (Bos et al., 2010). Support from the MHS in implementing LLL was proven effective in the past with the SLIM project (Wiefferink et al., 2005). Our study revealed that barriers and facilitating factors that influenced the implementation-promoting activities of the MHS were related to characteristics of the previous LLL program and the role of teachers, the school, the MHS and the municipality, as shown in chapter 2. According to the MHS professionals, the previous LLL program was considered outdated, too extensive and not a novelty among teachers. In addition, the MHS professionals mentioned that teachers considered relational and sex education unimportant or a difficult theme to discuss in the classroom, they struggled with their own norms and values and an inability to deal adequately with the difficult situations that they encountered in the classroom when teaching this subject. Finally, the MHS mentioned that schools did not integrate sexual and reproductive health (SRH) in their school policy, did not feel a need or did not have time for SRH and did not perceive LLL as compatible with the subjects they taught in school. The MHS themselves suffered from a shortage of time, difficulty finding a contact person and the appropriate contact moment within schools, insufficient support and collaboration within the MHS and sexuality not being a prioritized theme within the MHS. The municipalities were mentioned by the MHS to be forced to make cut-backs, sexual health was not a political priority and sexuality was not included in their public health policy.

Factors that influence the supportive role of the MHS are thus found on multiple levels, requiring not only a revised LLL program but a multi-level approach to facilitate implementation. This meant a new implementation strategy aimed at supporting the professional development of teachers, convincing municipalities and schools of the

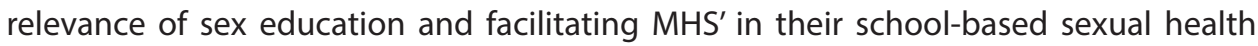
promotion activities. Despite the willingness of MHS professionals, the impact of the 2008 economic crisis limited the role of the MHS to stimulating the dissemination and adoption of LLL. While the MHS remains an important and competent party in the implementation of school-based sexual health education programs, this paper 
concludes that their restricted capacity, limited didactic skills and the teachers' need for ongoing support during real-life implementation necessitates an alternative form of support for teachers during implementation, possibly in the form of online coaching.

\section{Development of a web-based coaching intervention to support teacher implementation}

Chapter 4 proceeds with a description of the systematic development of the e-coaching website, 'lesgevenindeliefde.nl' or 'teachinglove.nl' as a specific component of the general implementation strategy. For school-based health promotion programs, teachers are key players in program implementation but teachers support in this phase is mostly limited to technical support and information (Rohrbach et al., 2010; Hansen et al., 2009). To support teachers with implementation of LLL with completeness and fidelity, a web-based coaching website was developed using the IM protocol (see chapter 4). An additional study was conducted among teachers $(\mathrm{N}=11)$ by means of interviews, as part of a needs assessment to develop e-coaching. This needs assessment was required to gain insight into teachers' implementation behavior and their perceived need and preferences for support in delivering SRH lessons, including LLL, with completeness and fidelity. The earlier two studies presented in chapters 2 and 3 showed that teacher's implementation behavior was characterized by inconsistently selecting parts of the program and not delivering (all) lessons as intended by program developers and this was reconfirmed in this needs assessment among teachers. This third study, however, revealed an interesting discrepancy: Teachers, however, did not perceive their teaching behavior as problematic and felt no need for coaching or support in teaching $\mathrm{SRH}$, despite their teaching behavior not meeting the required completeness and fidelity for program effectiveness. There was thus a discrepancy between actual need for coaching, according to program developers and teachers own perceived need for coaching in delivering LLL; as program developers, we understand the importance of completeness and fidelity for program effectiveness. However, we perceived that teachers' teaching behavior is not optimal as they are not implementing LLL with completeness and fidelity. We considered this problematic and felt they needed coaching to improve implementation quality. Teachers, however, do not perceive their teaching behavior as problematic; they do not perceive picking and choosing program components and their method of teaching as problematic and thus feel no need for coaching in teaching SRH generally or LLL specifically. The problem was therefore that despite their implementation behavior being suboptimal, according to program developers, teachers themselves did not perceive their teaching behavior as problematic and felt resistance to coaching or behavioral change. Several studies revealed that there is an implementation problem among teachers but teachers themselves do not perceive this 
suboptimal implementation behavior as a problem (van Lieshout et al., 2016; Buston et al., 2002). When asked about their need for support in delivering SRH, teachers mentioned a need for good teaching material they could use for their students. Teachers also acknowledged different difficulties they encountered when delivering LLL, which could potentially interfere with the quality of implementation. This is in line with what the MHS professionals reported as barriers to implementation at the teacher level in chapter 3.

Although teachers expressed a minimal need for coaching during the interviews in the needs assessment, program developers nonetheless saw the need to develop e-coaching for the following reasons: (1) teacher implementation was not optimal, despite teachers' conviction of their own teaching behavior. As program developers, we wanted to provide support so teachers could deliver LLL with completeness and fidelity. (2) Teachers could use support in adequately dealing with the difficult situations encountered when delivering SRH lessons, which could lead to improved program implementation. (3) A website is an efficient, low-threshold way of reaching a mass of teachers.

E-coaching was developed to improve teachers' implementation behavior through self-reflection and skills development. We attempted to address the discrepancy between teachers and program developers and bring about behavioral change by bridging the gap between perceived and actual need for coaching, using the technique of unobtrusive coaching, including self-reflection. We aimed at creating a need for coaching in teachers, without awakening resistance, by exposing them to difficulties experienced by other teachers they identified with, in the delivery of SRH. To create a 'need for coaching' or desire for 'behavioral change' meant creating awareness that their teaching behavior is not optimal when it comes to completeness and fidelity and that they need coaching in order to change this. Directive coaching would not have been appropriate as eagerness is a necessity before entering and being involved in the directive coaching process and these teachers were resistant to change (Crutzen et al., 2012; Fielden, 2005).

The e-coaching website was guided by theories of implementation behavior and developed to be an easily accessible and flexibly usable website, tailored to the needs of teachers. Using an online intervention is an easy and cost-effective way of reaching many teachers simultaneously. It was a by-and-for teachers approach; teachers were involved in the development of e-coaching, they informed and were a part of the 
content of the website in the form of teacher videos, role-model stories and tips and teachers were given the option of interacting with one other on the website in the form of posting comments.

E-coaching was aimed at influencing psychosocial determinants of implementation behavior, such as awareness, teachers' personal benefit, social support, (anticipated) student responses and self-efficacy to enhance completeness and fidelity. It consisted of a self-reflection tool which was used to create awareness of their need for coaching or behavioral change. Teachers could reflect on their teaching behavior, potential difficult situations they could encounter when delivering the lesson in the classroom and how to deal with them. To assist teachers in behavioral change, they were supported in dealing adequately with difficult situations encountered when delivering SHR lessons, such as creating a safe atmosphere in the classroom for students to openly discuss sexuality. The support was provided through role model stories, video's, tips, background information, and FAQ's The website also included student materials and a downloadable and printable teacher manual with additional information and suggestions on the LLL program to further enhance the delivery of LLL with completeness and fidelity. As described at the beginning of this discussion section, E-coaching was integrated as part of a broader implementation strategy to stimulate the implementation of LLL with completeness and fidelity among teachers.

\section{Effect and Process evaluation of the web-based coaching intervention 'Lesgevenindeliefde.nl'}

An effect evaluation was performed to evaluate the effectiveness of the e-coach, in influencing (determinants of) teachers' implementation (see chapter 5). A cluster randomized controlled trial (e-coaching vs waiting list control) was conducted with a baseline assessment (T0) and follow up (T1) two weeks after completing the LLL program. Teachers in the intervention and control group both received the LLL program, a teacher manual and the option to receive an MHS training prior to program implementation. Teachers in the intervention group, however, had exclusive access to the e-coaching website during the study. A total of 43 schools with 83 teachers participated in the study. In the follow-up 38 schools participated; 23 in the e-coaching condition with 41 teachers, 15 in the control condition with 26 teachers. Teachers' implementation behavior was measured based on rates of completeness and fidelity of LLL implementation. In addition, the effects of the e-coach on determinants of implementation behavior were evaluated. These determinants were targeted by the e-coach, namely teacher benefits, subjective norms, social support, self-efficacy and (anticipated) student response. 
E-coaching was not found to be effective in enhancing completeness and fidelity of LLL by teachers ( $p$ 's > .60; $d$ 's $<.17$ ), neither on positively influencing any of the determinants of successful implementation ( $p$ 's $>.22$; $d$ 's $<.33$ ). The lack of effect was attributed to the intervention content, the limited use, and/or the study design itself. Despite being aimed at determinants of completeness and fidelity, the e-coaching intervention may not have addressed the exact needs of the target population or been able to increase teachers' awareness of the importance of completeness and fidelity or did not address fidelity and completeness sufficiently to bring about behavioral change. Besides, the e-coaching intervention was not or insufficiently used by teachers. Furthermore, the study had a possible sample bias of experienced, motivated teachers who were already capable of delivering LLL successfully and a 'ceiling effect', with the implementation grade of teachers participating in the study already being high, making it difficult to improve using the e-coaching intervention. A process evaluation was executed to investigate teachers' appreciation, use and (motives for) use of the e-coach to further understand why e-coaching was not used and had no effect.

The process evaluation outcomes (presented in chapter 6) were in line with the hypothesized explanations for lack of effect of e-coaching. Although Lesgeveindeliefde. $\mathrm{nl}$ was perceived by teachers as usable and as an instrumental addition to the LLL program, its personal relevance was rated low. The lack of effect could be explained by non- or insufficient use of the website, limitations of the intervention itself and the research design, as hypothesized in the effect evaluation. These aspects are elaborated on below.

The suboptimal use of e-coaching is likely the main reason why it did not have any significant effects on implementation behavior of teachers or on the related determinants. Of the 41 teachers in the intervention group invited to use the e-coach, 30 actually visited the website (75\%), of which the majority did so 2 to 3 times $(n=20$; $66.7 \%)$. The extent of e-coaching use by teachers was limited with only $13.3 \%(n=4)$ of teachers having viewed all components of the website.

Lack or non-use of the website according to teachers was due to a lack of time, the introduction of two innovations at once - LLL and Lesgeveindeliefde.nl - and lack of perceived personal relevance of the website due to unmet expectation of finding student material to use in the classroom-'I didn't find what I was looking for'-, extensive experience teaching SRH thereby not finding new information on the website - 'I already know this' and they lacked a perceived need for coaching - 'useful, but not for me'. A systematic review of reviews on online prevention for lifestyle behaviors revealed 
that overall, effects are small, variable, and not sustainable and that the determinants of effectiveness are unclear. The reach and use of these online interventions were also not ideal and needed more research (Kohl, Crutzen, \& de Vries, 2013).

This lack of perceived need for coaching was already established in the needs assessment phase of the development of e-coaching and reconfirmed in the process evaluation. Teachers felt no need for coaching because they were convinced of their SRH teaching methods- 'I'm teaching well'- and they did not perceive their implementation behavior of picking and choosing program elements as problematic. Teachers therefore appeared to have limited awareness of the implementation problem and did not seem to understand the importance of completeness and fidelity in delivery of SRH lessons.

Limitations of e-coaching intervention itself were therefore that it seemed unable to change teachers' perceived need for coaching. It may have insufficiently addressed the importance of completeness and fidelity and did not address direct, practical means of achieving completeness and fidelity of LLL enough. Instead, it focused predominantly on dealing with difficult situations, which are the preconditions required to deliver $\mathrm{SRH}$ lessons with completeness and fidelity. Additionally, the self-reflection tool was largely inaccessible due to technical problems- while that was a necessary first step to create awareness of own teaching behavior. Consequently, although the e-coaching intervention contains elements to create the prerequired conditions for completeness and fidelity, in its current form, the e-coach may not have been ideal due to perhaps containing insufficient content to directly influence completeness and fidelity of LLL specifically.

Based on these findings we realized in hindsight, after analyzing the data collected in the process evaluation, that the discrepancy that exists between teachers and e-coach developers on the perceived need for e-coaching or behavioral change is related to a different interpretation regarding quality of teaching. For teachers, 'correct' program delivery and thus good teaching of SRH is related to having teaching material for students and sufficient didactic skills to deliver lessons. For program developers, however, 'correct' program delivery is related to implementation with completeness and fidelity. Teachers and program developers were thus approaching the problem from different reference frameworks and both were right from their own perspectives: Teachers felt they were teaching well because they were thinking of their teaching behavior from their reference framework of didactics, which is their profession and expertise, and thus did not perceive a need for teaching support. They were not thinking in terms of completeness and fidelity, as program developers were. This could explain why perceived need for coaching remained unchanged, even after exposure 
to e-coaching: teachers may not have been thinking of completeness and fidelity as a component of good teaching and did not seem to understand its importance in the implementation of LLL. As a result, the e-coach lacked personal relevance and was not used by a majority of teachers.

\section{Methodological considerations: weaknesses and strengths}

There are some limitations to the studies presented in this dissertation that are important to consider. Although IM was useful in designing the web-based coaching intervention, it is a time-intensive method, sometimes restricted by limited resources of time and money. Mistakes can be made when executing each step of the protocol which in turn influences the next steps; therefore it is not a guarantee for an effective intervention. Also, it is often difficult to prove the effectiveness of interventions, even if they are solidly grounded in theory and evidence (Bartholemew et al., 2016). The iterative nature of the process and an evaluation, however, are crucial for checking each step and understanding obtained results. Conducting an effect-and process evaluation during this project proved useful for understanding the research outcomes and reinforces the importance of evaluating interventions to then further improve them.

It is only in hindsight, after analyzing the data collected in the process evaluation, that we realized that there was a discrepancy in the meaning of 'correct program delivery' between program developers and teachers already from the start of the project. Each party referred to 'teaching $\mathrm{SRH}^{\prime}$ and 'need for coaching' from their own reference framework: that of completeness and fidelity for the developers and that of didactics for the teachers, respectively. Teachers' reference framework guided the development of e-coaching as the content was based on the aspects teachers mentioned they needed support in. As a result, e-coaching focused predominantly on the didactics of dealing with difficult situations, which formed barriers to delivering SRH generally, and focused less on completeness and fidelity of LLL specifically. Although e-coaching contributed to completeness and fidelity of LLL by supporting teachers in creating the preconditions required to deliver $\mathrm{SRH}$, in its current form, the e-coach may not have included sufficient content to address completeness and fidelity of LLL directly, rendering it less ideal. Because a mutual understanding of what 'correct program delivery' is was lacking, however, the important first step of highlighting the importance of completeness and fidelity and specifying it as a component of 'good teaching' of $\mathrm{SRH}$, prior to coaching, was insufficiently addressed in e-coaching and in the broader implementation strategy. Limited use of the website followed and a lack of effectiveness. 
Although the implementation strategy described in this dissertation considered the individual teacher as well as the broader environment (i.e. schools, MHS and municipality), the focus was predominantly on individuals within these organizations that are actually executing the tasks, with bottom-up influence. For example, teachers delivering LLL or MHS professionals executing the implementation promotion activities. The lack of congruency, capacity, communication and policies within schools and MHS organizations result in dependency on enthusiastic individuals rather than sustainable organizational support. Therefore, to strengthen the sustainability of LLL implementation, more attention is needed for decision-making processes in schools and organizations to influence top-down policy formation at the management level (Forman et al., 2009).

Dependency on the MHS for the delivery of the implementation strategy was a strength in that it made use of existing networks but was also restricting. Although a uniform approach was supported as much as possible, it could not be guaranteed. The implementation strategy was, however, not fully dependent on the MHS as direct support was also provided to teachers by program developers by means of newsletters etc.

Some limitations in the various studies conducted should also be mentioned. For the determinant study (chapter 2), the main limitation is the cross-sectional nature of the data, which does not allow drawing conclusions about causality. Self-report by teachers and a self-selected sample formed an additional methodological limitation (Lillehoj, Griffin, \& Spoth, 2004). Observation of fidelity by an external party would have further validated the results of this study. The qualitative and quantitative study focusing on MHS (chapter 3) also included self-reported data in which MHS professionals had to report their predictions for the new LLL program, that was not yet available at the time of that study. Their predictions may therefore not have been complete. There may also have been a sample bias, with the MHS professionals who were more optimistic about LLL having participated in the study. This may affect the generalizability of the results.

The RCT design in chapter 4 included a baseline measure and post-test, yet the number of participants that completed both surveys was relatively low. This could have affected the generalizability of the results. Moreover, a 'ceiling effect' could be present due to the implementation grade of teachers participating in the study already being high, making it difficult to improve using the e-coaching intervention. Finally, the teachers who agreed to participate in the study may have been a biased sample of motivated, experienced teachers who were already capable of delivering LLL successfully. The same applies to the teachers who participated in the process evaluation study, emphasizing 
the feeling of no need for e-coaching. Although the experimental setting allows for controlled intervention delivery, evaluation in the real-life setting over time is necessary to evaluate and monitor changes in use and appreciation of the intervention.

Primarily, the strength of this dissertation lies in the systematic approach that was used in planning for, developing and evaluating the implementation strategy, using the IM protocol. This protocol required theory-and evidence-based decision making in program development, involvement of the target group and intermediaries and consideration of the environmental level, to bridge the gap between research and practice (Domitrovich et al., 2008). It proved to be a useful tool for guiding the scientific development of interventions and making them compatible with the needs and preferences of the target group. It exemplified the ongoing process of balancing input from the target group with the desired goals of the intervention developers to ultimately develop a theory and evidence-based intervention. With e-coaching being an innovation, IM assisted in the process of learning-by-doing. The results of this dissertation are applicable to the Dutch context for implementation of school based (sex) education programs but are also generalizable to the implementation of other health promotion programs in the school setting. Due to the theoretical and empirical approach, the methodology used and the results are also generalizable to other settings where implementation of health promotion takes place such as organizations and health care institutions (Bartholemew et al., 2016). A great deal of attention was dedicated to the implementation of LLL in this project, which is an exception to what most organizations can afford qua time or budget.

By anticipating for implementation during development of LLL, intervening actively in the implementation process and investing in professional development in this project, we increased the likelihood of successful implementation of LLL and in turn reach the target group and have impact on their sexual and relational health. Involving teachers and MHS professionals from the start increased the likelihood of sense of ownership and of developing an intervention and implementation strategy compatible with the desires and capabilities of the target group, and the intervention context. LLL, for example, was limited to six lessons and was sensitive to gender, sexual, and cultural diversity, compatible with working methods of teachers, being a ready-made flexible teaching pack to be used in different classrooms. Choices and concessions were made to come to the final program by keeping implementation in mind. The likelihood of delivery of LLL with completeness and fidelity was therefore greatly increased by anticipating implementation during the development of LLL and creating a program that solely required facilitation of the teacher in the delivery of LLL, rather than being overly dependent on them for the quality of implementation. Additionally, we 
actively intervened in the implementation process with theory- and evidence-based implementation strategies, trainings and online support. Different strategies were developed and used to promote each stage of the implementation process, not only dissemination and adoption, as most other work seems to have focused on (Bessems et al., 2014; Domitrovich \& Greenberg, 2000; Fagan \& Mihalic, 2003; Durlak, 1998). Formal and informal media and communication channels were used, which can enhance the durability of an innovation and ensure its long-term continued use (Oldenburg \& Parcel, 2002). Teachers, who are key figures in the delivery of school-based sex education programs, vary widely in their actual adoption and implementation behavior. They were supported through trainings and e-coaching to reflect on their teaching behavior, to adequately deal with difficult situations and to implement LLL with completeness and fidelity. The support teachers received via e-coaching, to create the prerequired conditions to deliver SRH, formed a component of what is needed to deliver SRH and LLL with completeness and fidelity, alongside the availability of the LLL program.

In addition, the findings are based on the results of both quantitative and qualitative approaches, with the added advantage of data triangulation to enhance the validity of the findings. A randomized controlled trial was conducted to determine the effects of this web-based coaching intervention. Although often overlooked in research, this RCT was followed by a process evaluation, which allowed for a better understanding of the outcomes (Bartholemew et al., 2016).

This dissertation also contributes to the implementation research focused on webbased coaching interventions to support teachers' implementation of the school-based sex education programs. Support during the implementation phase of school-based health promotion programs is currently limited to providing practical support in the form of teacher manuals with practical information on the content of the lessons and on how to deliver such lessons. However, more in-depth coaching focusing on determinants of implementation such as self-efficacy and social support to enhance completeness and fidelity was lacking (Hansen \& Bishop, 2009; Rohrbach et al., 2010; Bos et al., 2010). In developing the implementation strategy, we conducted a determinant analysis beforehand and applied the results to the implementation strategy. As a result, systematically designed strategies were developed as part of the implementation strategy that were tailored to an empirically based selection of determinants. Many innovation strategies applied are often not based on theory (theoretical methods for change), which may result in the use of inappropriate, and thus ineffective, strategies, wasting time and money (Fleuren et al., 2004). E-coaching was aimed at specific determinants, as was the teacher training. The strength of e-coaching itself is that it 
is more than just a one-time training. Instead it provides assistance during real-life implementation situations and has a longitudinal character in that teachers can visit the website when they want or need to (Hann 2005).

The implementation strategy was also not exclusively aimed at behavioral change on the individual level, but considered the broader environment in which the behavior takes place by involving the MHS, for example (Bartholemew et al., 2016). The MHS were well represented in the data with most regions participating in the research. This makes the outcomes of the research generalizable to all MHS' in the Netherlands.

\section{Implications, recommendations and future research}

The discrepancy discovered between teachers and program developers' perceptions of 'correct program delivery' of SRH during the process evaluation of e-coaching was a revelation. It not only aided in potentially explaining the lack of use and effectiveness of e-coaching, but is fundamental to implementation of all interventions in school-based settings involving teachers. As researchers and program developers we were reminded that we operate from a particular reference framework, but so does our target group and sometimes program development can be misguided when both parties are not 'speaking the same language.' Program developers need to ensure that they and their target group are on the same page before attempting to change behavior. In the case of $L L L$, we realized teachers are experts in didactics but they still need to improve their completeness and fidelity of LLL program delivery. Teachers, however, appeared to approach SRH delivery from the reference framework of didactics, which was reflected in their need for student material and need for support in dealing with difficult situations, for example, rather than from the perspective of completeness and fidelity and therefore felt no need for coaching. Due to their focus on didactics and viewing themselves as professionals in their field, teachers also tended to think mainly in terms of materials they could use for their students rather than reflect on their own teaching behavior. This expectation and need for student material to use in the classroom was also found, for example, in a study among teachers who taught students with different learning abilities (Lane, Mahdavi, \& Borthwick-Duffy, 2003). Now that we have realized that we were approaching the problem from different reference frameworks, we need to create a mutual understanding of what 'correct' program delivery is to ensure we are 'speaking the same language'. In order to achieve this, teachers' professionalism and reference framework of good teaching, involving didactics, needs to be acknowledged and respected by program developers, while teachers need to be made aware of the implementation problem as program developers perceive it. 
Recommendation: To reach a mutual understanding of 'correct' program delivery and to stimulate the personal relevance of coaching or behavioral change, teachers most likely first need to understand the importance of completeness and fidelity for program effectiveness when delivering SRH and perceive it as a component of good teaching of SRH. For LLL, teachers need to understand that they need to use all the program components and deliver all the lessons according to the teacher guidelines, and understand that it is important and why it is important that they do this, namely to achieve program effectiveness. The e-coaching intervention itself can include this information and the broader implementation strategy can also be utilized to raise this awareness via MHS trainings or information leaflets, for example. Teachers can subsequently be supported to improve the quality with which they implement LLL and other SRH programs. This is possible through e-coaching which enhances professional development by providing tools to assist them in reflecting on their teaching behavior, in dealing adequately with difficult situations that arise in the classroom and delivering LLL with completeness and fidelity. Lack of awareness of the importance of completeness and fidelity was found to be related to incomplete program implementation of the online school-based sex education program, LLL+ for older high school students (van Lieshout et al., 2016). Previous research has shown that teachers' incomprehension of the theoretical basis for behavior change negatively influences implementation (Buston et al., 2002; Shepherd et al., 2014). Coaching can only commence when teachers develop an awareness of the need and desire to improve their performance or change the way they have been doing things at work (Parsloe \& Leedham, 2009).

Future research: The discrepancy found between teachers' perceived and actual need for coaching was due to teachers and program developers differing perceptions of 'correct program delivery' or 'good teaching'. We therefore assumed that teachers are not aware of the importance of completeness and fidelity. Research investigating this discrepancy is, however, advised. Teachers should be asked whether they perceive completeness and fidelity as important, what their perceptions are of correct program delivery and how they can be supported in implementing LLL specifically with completeness and fidelity. These insights can then be incorporated into the implementation strategy to improve implementation quality.

Another realization we had, as a result of the effect and process evaluation, was that although completeness and fidelity are absolutely important for program effectiveness, when it comes to teaching SRH, good program delivery encompasses more than just delivering lessons with completeness and fidelity. It also includes the ability to create prerequired conditions which make it possible to deliver these lessons with completeness and fidelity. Teachers are therefore absolutely right in their perceptions of teaching well, 
which includes didactic skills. Their professionalism in their field and perception needs to be acknowledged and supported alongside promoting completeness and fidelity. As program developers, we are after all dependent on their cooperation to implement our intervention. When supporting teachers in the delivery of $\mathrm{SRH}$, program developers therefore need to focus on more than just means of directly enhancing completeness and fidelity. To successfully deliver sex education, not only are a quality program and tools needed to implement that program with completeness and fidelity but support in creating the prerequired conditions in which it is provided, for example, in a safe and trusted environment, and the ability to deal adequately with difficult situations that arise, as well as the didactic skills, are essential for successful program delivery and resulting effectiveness (Vanwesenbeeck et al., 2015). This realization was supported by the findings of the effect evaluation of LLL among students. LLL was found to be effective and interestingly 'Lesgevenindeliefde.nl' resulted in additional positive effects at the student level; students of teachers who used 'Lesgevenindeliefde.nl' during their implementation of LLL evaluated the components of the program and atmosphere in the classroom more positively (Hofstetter et al., 2014). So although no effects were found on completeness and fidelity at the teacher level, students' experience of the LLL lessons appeared to be enhanced when teachers were exposed to e-coaching. Teachers therefore not only need to be supported in delivering SRH with completeness and fidelity but also in creating the prerequired conditions and in dealing with difficult situations as part of their professional development, as was attempted with the e-coaching intervention. Implementation of LLL with completeness and fidelity thus requires more than just e-coaching. It can be achieved through the LLL program itself, due to it being developed in a manner that promotes completeness and fidelity, through supporting teachers in delivering LLL with completeness and fidelity, through supporting teachers in creating the preconditions required to deliver $\mathrm{SRH}$, as was attempted with e-coaching and by utilizing the broader implementation strategy to raise awareness and stimulate adoption and continuation as well.

Recommendation: In its current form, the e-coach may not have been ideal for directly influencing completeness and fidelity of LLL specifically due to a focus mainly on removing the barriers for teaching $\mathrm{SRH}$ in general and creating the prerequired conditions for completeness and fidelity of SRH generally. It was not explicit enough in stating the importance of completeness and fidelity and explaining what should be done and why. Other than including information emphasizing the importance of completeness and fidelity, e-coaching would thus also need to include tools to enhance fidelity and completeness of LLL specifically. For example, a video of other teachers explaining how to use the teacher manual and deliver all six lessons of LLL within a particular period of time. Or guidelines in the teacher manual to deliver principal components of the LLL 
program to preserve effectiveness. The self-reflection tool, which is essential in creating awareness of own teaching behavior, will need to be optimized into a shorter, more interactive quiz, for instance. Exploring other means of promoting self-reflection is also possible, for example during teacher trainings. E-coaching is only one part of the broader implementation strategy and on its own is not enough to enhance completeness and fidelity of LLL. The LLL program itself as well as the broader implementation strategy are also necessary.

Next, use of the implementation enhancing intervention by teachers needs to be encouraged. Especially website use in school-based settings is particularly challenging. A large study in the Netherlands found that teachers in secondary schools either consult colleagues in their schools for information or use the internet mainly to find information, prepare their lessons, send e-mails to students or give homework assignments and thus less for professional development (TNS, NIPO, 2005). Voogt et al., (2016) further highlighted the limited use of IT by teachers in Dutch school-based settings. Limited use of online interventions in the school-setting, however, does not automatically imply that web-based coaching in itself is an ineffective strategy to support delivery of health promoting programs in schools. Digital technologies are being increasingly used in the education system, bringing exciting opportunities for innovative ways of teaching and learning (Ertmer \& Ottenbreit-Leftwich, 2010). Exploring the educational potential of these digital technologies and supporting schools in making use of them remains important (Ertmer \& Ottenbreit-Leftwich, 2010). The MHS, as external party, therefore plays an essential role in the broader implementation strategy with their implementation-promotion activities, including promotion of website use.

Recommendation: Use of the e-coach could be improved. If the website itself is not used, it cannot have effect. Website use was found to be related to factors associated with the visitor (such as their motivation to be healthy) (Crutzen et al., 2008; Riet et al., 2010) as well as the intervention website (such as offering tailored information) (Brouwer et al., 2011; Crutzen et al., 2011; Nijland et al., 2011). Increasing the personal relevance of the website is necessary to get teachers to the website and keep teachers on the website and prevent discontinued use. Low personal relevance was fundamental to limited use of website: the website was not perceived as personally relevant because teachers felt no need for coaching and as a result did not use the website. This lack of personal relevance, related to a lack of need for coaching, stems from teachers' reference framework of correct program delivery being related to didactics rather than completeness and fidelity. Increasing personal relevance of a website was found to be a promising strategy to increase use of internet-delivered interventions (Crutzen et al., 2014). We therefore need to increase e-coaching's' personal relevance (and create a need for coaching or 
behavioral change) by highlighting the importance of completeness and fidelity for program effectiveness and specifying it as a component of good teaching. This will provide a motive to visit the website. The broader implementation strategy should then be utilized to bridge the intention-behavior gap and promote actual website use. The implementation activities should be deployed such as distributing information leaflets and supporting the MHS in the provision of teacher trainings. The process of accepting the innovation such as e-coaching takes time, as described in Rogers' diffusion curve (Rogers, 2003). The innovation will most likely be adopted by innovators first, followed by the early majority and eventually the laggards. Pijpers et al., (2002), claim that online innovations first need to be accepted in order to be used broadly and effectively. The options of web-based coaching should be explored further as it remains a low-threshold, cost-efficient manner to reach many teachers.

Although completeness and fidelity are important, the fact that almost no schoolbased program seems to be delivered with completeness and fidelity brings into question whether LLL or other school-based (sex) education programs can realistically be expected to be delivered with absolute completeness and fidelity as program developers require (Durlak \& DuPre, 2008). Adaptations often occur in the form of omission or additions to adapt to the local situation and are often viewed as program failure by program developers rather than potential improvements (Miller-Day et al., 2013). These adaptations are sometimes inevitable, requiring adaptation of program components or teaching methods to accommodate changing circumstances in schools and diversity in classroom composition (gender, ethnicity or sexual experience of students) (Durlak, 2013). Adaptations that enhance ownership and commitment or support goodness of fit between program and setting may, however, be beneficial by facilitating implementation and improving sustainability, and effectiveness. Keeping these adaptations in mind and considering that absolute completeness and fidelity cannot realistically be expected, teachers should be supported in delivering programs with as much completeness and fidelity as possible, with guidance in delivering principle program components to ensure program effectiveness is preserved (Han \& Weis, 2005). This requires creativity from program developers to accommodate teachers as well as preserve completeness and fidelity as much as possible.

Recommendations: This can partly be accomplished by anticipating for implementation during program delivery, accommodating the intervention as much as possible to the needs and working methods of teachers, and investing in the professional development of teachers. A program that is easy to use, flexibly deployable, compatible with teaching methods and requiring teachers to simply be facilitators rather than having to invent their own methods of teaching, increases the likelihood of quality implementation. Providing 
teachers with the tools and skills required to create the classroom conditions that enable quality delivery of SRH lessons with completeness and fidelity, and guidelines in making choices to implement the essential program components for the most positive effect possible given a certain context, should assist in successful implementation. Despite improving their professional development, however, environmental factors outside the teacher and in the school setting and MHS organization, such as capacity and time shortage, may continue to form barriers to teacher implementation (Ahtola et al., 2013, Bessems et al., 2011; Wind et al., 2008; Buston et al., 2002). These environmental factors including school policy, culture, support and socio-political context form the prerequisites for successful implementation and need nonetheless to be taken into consideration when thinking of promoting implementation.

Future research: The extent to which implementation of Long Live Love by teachers is linked to effectiveness of the program among students should be investigated. This will shed light on the importance and extent of delivery of LLL with completeness and fidelity required to achieve effect.

In addition, means to further improve e-coaching and attract teachers to the website should be explored as well as how flexibility of the program can be used to support teachers with completeness and fidelity.

Finally, implementation of implementation enhancing interventions, such as e-coaching is not guaranteed. To avoid transference of the implementation problem, planning is needed.

Recommendation: A broader implementation strategy is needed, not only to promote website use but also to stimulate adoption, implementation and continuation of LLL. The MHS certainly remains an important party for promoting school-based interventions. Their limitations, capabilities and possibilities always do need to be taken into account when defining their implementation-promoting role, with the awareness that this role could be influenced by factors on socio-political, municipal and organizational level. Direct support of teachers by program developers is therefore also still advised.

Promoting sustainability of LLL and other school-based sex education programs could assist implementation of implementation-enhancing interventions. STI Aids Netherlands is responsible for the development and implementation of interventions for the promotion of sexual health of the youth and in schools. STI Aids Netherlands will embed the implementation strategy described in this dissertation in their activities and maintain contact with the MHS and continue monitoring use and evaluations of 
LLL among teachers and students to adapt and expand it to changing circumstances to ensure continued use. Constant collaboration and involvement of the target group and intermediaries such as teachers and MHS professionals is necessary. Systematic development of new interventions and adaptation of existing ones using IM is recommended for highest effectiveness. For future sustainability of LLL, STI Aids Netherlands could also incorporate LLL in the national overview of prevention programs for the school-based setting and integrate the program in national databases of network organizations such as the Health Promoting School ('de Gezonde School'), and introduce the program in the education of teachers, while still in training (Forman et al., 2009). The Ministries of Health and Education need to be stimulated to formulate local and national policies that will ensure the integration and structural embedment of sex education in schools, and in the MHS. School policy and supportive school management are necessary to formally establish sexual health education in schools. The Healthy School approach, encouraging a whole school-approach may be beneficial to sustainability.

Future research: This dissertation focused on teachers at the individual level and the MHS professionals at the environmental level. Barriers and facilitating factors were identified at both the individual and environmental level and were addressed as adequately as possible in the current implementation strategy. The next aspect to focus on would be researching and influencing factors associated with stimulating continuation and assuring sustainability of LLL in Dutch schools at the organizational and national level (Forman et al., 2009). We recommend research on decision-making processes in schools and within the MHS and their related municipalities in order to assist schools in the development of a policy to integrate LLL as a fixed part of the curriculum, and incorporate sexual health promotion and implementation promoting activities of LLL in the work plans of MHS professionals. This next level is required to warrantee long term implementation of LLL.

\section{GENERAL CONCLUSION}

The focus of this dissertation is on enhancing the implementation of Long Live Love to ultimately improve the sexual health of the Dutch youth. The complexity of ensuring successful implementation was once again confirmed in this dissertation by conducting the research, developing the implementation strategy and evaluating the effects of the e-coach website specifically as part of the implementation strategy. 
Constant collaboration and involvement of the target group and intermediaries, such as teachers and MHS professionals, and combining theory, research and practice form the foundation for developing any implementation strategy.

Guiding the implementation process and changing implementation behavior of intermediaries such as teachers and MHS professionals is not a simple task or automatic process. It requires anticipation for implementation during program development, actual systematic development of different theory- and evidence-based strategies at the individual and environmental level to promote each step of the implementation process and continued investment in professional development. Intervention Mapping is a useful tool to ensure timely planning and bottom-up development of an implementation strategy.

Achieving completeness and fidelity of SRH program delivery is a puzzle composed of several components. In the case of LLL, more than just e-coaching is required. E-coaching is one component of a broader implementation strategy that is needed to support the entire implementation process of LLL. The likelihood of achieving completeness and fidelity of LLL can be increased by: a) A mutual understanding of 'correct' program delivery between program developers and teachers, b) Awareness among teachers of the importance of completeness and fidelity; that it should be done and why it is important and that it is also a component of teaching well, c) A well-developed program as a tool, in this case LLL d) Support in delivering LLL specifically with completeness and fidelity e) Support in creating the prerequisite conditions to effectively deliver SRH generally, such as how to create a safe environment in the classroom to discuss sexuality and f) A broader implementation strategy to stimulate the implementation process of LLL.

Despite these efforts, in real life, interventions are often adapted to fit the classroom circumstance and this should be anticipated for by program developers. Keeping these adaptations in mind and considering that absolute completeness and fidelity cannot realistically be expected for school-based health promotion programs, teachers should be supported in delivering programs with as much completeness and fidelity as possible, with guidance in delivering principle program components to ensure program effectiveness is preserved. Environmental factors outside the control of teachers also influence the degree to which a program can be implemented and need to be taken into consideration. Continuous changes within organizations and in the environmental context call for continuous adaptation of the implementation strategy and the LLL 
intervention itself to actuality, changing circumstances, the needs and desires of program users and implementers and to the intervention context in order to ensure its continuation. This exemplifies implementation as a dynamic process.

A qualitatively sound, attractive and effective intervention such as Long Live Love should continue to be disseminated to schools and implemented by teachers. MHS professionals and teachers need to continue being supported in their implementation of the implementation strategy and LLL respectively, so that ultimately, the Dutch youth is better prepared, motivated and able to practice safe sex and have mutual, pleasant relations. Long Live Love! 
References 



\section{REFERENCES}

Ahmed, N., Flisher, A. J., Mathews, C., Jansen, S., Mukoma, W., \& Schaalma, H. (2006). Process evaluation of the teacher training for an AIDS prevention program. Health Education Research, 21(5), 621-632. https://doi.org/10.1093/her/cyl031

Ahtola, A., Haataja, A., Karna, A., Poskiparta, E., \& Salmivalli, C. (2013). Implementation of antibullying lessons in primary classrooms: How important is head teacher support? Educational Research, 55(4), 376-392. https://doi.org/10.1080/00131881.2013.844941

Ajzen, I. (1988). Attitudes, personality and behavior. Milton Keynes: Open University Press.

Ajzen, I. (1991). The theory of planned behavior. Orgnizational Behavior and Human Decision Processes, 50, 179-211. https://doi.org/10.1016/0749-5978(91)90020-T

Ajzen, I. (2011). The theory of planned behavior: Reactions and reflections. Psychology \& Health, 26(9), 1113-1127. https://doi.org/10.1080/08870446.2011.613995

Bakker, F., Fulpen, M. van., Paulussen, T., Vanwesenbeeck, I., Wiefferink, I., Linthorst, M., Poelman, J., \& Wijngaarden, J, V. (2002). Evaluatie van het SLIM project. Samenvatting van de resultaten van het onderzoek naar de implementatie en effectiviteit van het vernieuwde lespakket "Lang leve de liefde." Rutgers Nisso Groep, TNO-Preventie en Gezondheid.

Bandura, A. (1986). Social foundations of thought and action: A Social Cognitive Theory. New Jersey: Prentice-Hall.

Bartholomew, L. K., Parcel, G. S., Kok, G., Gottlieb, N.H., \& Fernández, M. E. (2011). Planning health promotion programs: An Intervention Mapping approach (Third). San Fransisco: Jossey-Bass.

Bartholomew, L.K., Markham, C. M., Ruiter, R.A. C., Fernàndez, M. E., Kok, G., \& Parcel, G. S. (2016). Planning health promotion programs: An Intervention Mapping approach (Fourth). Hoboken: Wiley.

Berman, P., \& McLaughlin, M. (1976). Implementation of educational intervention. The Education Forum, 40, 345-370.

Bessems, K. M. H. H., Van Assema, P., Martens, M. K., Paulussen, T. G. W. M., Raaijmakers, L. G. M., \& de Vries, N. K. (2011). Appreciation and implementation of the Krachtvoer healthy diet promotion program for 12- to 14- year-old students of prevocational schools. BMC Public Health, 11(1), 909. https://doi.org/10.1186/1471-2458-11-909

Bessems, K. M., van Assema, P., de Vries, N. K., \& Paulussen, T. W. (2014). Exploring determinants of completeness of implementation and continuation of a Dutch school-based healthy diet promotion program. International Journal of Health Promotion \& Education. Taylor \& Francis. https://doi.org/10.1080/14635240.2014.912445

Bishop, M., Reiser, S., Hall, J., Rein, J., \& Taylor, A. (2006). The hospice model of care: a required webbased program for medical students. Journal of Palliative Care, 22, 219-219. 
Boot, N. M. W. M., van., Assema, P., Hesdahl, B., Leurs, M., \& de Vries, N. K. (2010). Gezondheidsbevordering en voortgezet onderwijs: verstandshuwelijk of echte liefde? Tijdschrift Voor Gezondheidswetenschappen, 88(3), 127-135.

Borko, H., Livingstone, C., \& Shavelson, R. (1990). Teachers' thinking about instruction. Remedial and Special Education, 11, 40-9.

Bos, V., de Jongh, D., \& Paulussen, T. (2010). Gezondheidsbevordering en preventie in het onderwijs. Stand van zaken, effectiviteit en ervaringen van GGD'en en scholen. Retrieved from http://www. rivm.nl/dsresource?objectid=rivmp:11705\&type=org\&disposition=inline\&ns_nc=1

Bron, J., Loenen, S., Haverkamp, M., \& van Vliet, E. (2015). Seksualiteit en seksuele diversiteit in de kerndoelen. Een leerplanvoorstel en voorbeeldlesmateriaal. SLO (nationaal expertisecentrum leerplanontwikkeling), Enschede. Retrieved from

http://downloads.slo.nl/Repository/seksualiteit-en-seksuele-diversiteit-in-de-kerndoelen.pdf

Brouwer, W., Kroeze, W., Crutzen, R., De Nooijer, J., De Vries, N. K., Brug, J., \& Oenema, A. (2011). Which intervention characteristics are related to more exposure to internet-delivered healthy lifestyle promotion interventions? A systematic review. Journal of Medical Internet Research, 13(1), 1-19. https://doi.org/10.2196/jmir.1639

Burak, L. (1994). Examination and prediction of elementary school teachers' intentions to teach HIV/AIDS education. Aids Education and Prevention, 6, 310-321.

Buston, K., Wight, D., Hart, G., \& Scott, S. (2002). Implementation of a teacher-delivered sex education program: obstacles and facilitating factors. Health Education Research, 17(1), 5972. https://doi.org/10.1093/her/17.1.59

Campbell, B., \& Lubben, F. (2003). The provision of a health promoting environment for HIV/ AIDS education: The case of Namibian senior secondary schools. International Journal of Educational Development, 23(5), 529-542. https://doi.org/10.1016/S0738-0593(03)00058-0

Connell, D., Turner, R., \& Mason, E. (1985). Summary of the findings of the School Health Evaluation: Health promotion effectiveness, implementation and costs. Journal of School Health, 55, 316-21.

Cordingley, P., Bell, M., Rundell, B., Evans, D., \& Curtis, A. (2003). The impact of collaborative CPD on classroom teaching and learning. How does collaborative Continuing Professional Development (CPD) for teachers of the 5-16 age range affect teaching and learning? London. Retrieved from http://www.curee.co.uk/files/publication/1246025680/How does collaborative CPD for teachers of the 5-16 age range affect teaching and learning.pdf

Cordingley, P., Bell, M., Thomason, S., \& Firth, A. (2005). The impact of collaborative continuing professional development (CPD) on classroom teaching and learning. How do collaborative and sustained CPD and sustained but not collaborative CPD affect teaching and learning? London. Retrieved from http://www.curee.co.uk/files/publication/1246025289/The impact of collaborative CPD on classroom teaching and learning.pdf 
Coyle, K., Firpo-Tiplett, R., Taylor, J., \& Rex, P. (2011). Draw the line/respect the line Adaptation Kit. Scotts Valley. Retrieved from http://recapp.etr.org/recapp/documents/programs/DTL_ Adaptation_Kit.pdf

Crutzen, R., Cyr, D., \& de Vries, N. (2012). The role of user control in adherence to and knowledge gained from website: randomized comparison between a tunnelled version and a freedomof-choice version. Journal of Medical Internet Research, 14(2), e45.

Crutzen, R., de Nooijer, J., Brouwer, W., Oenema, A., Brug, J., \& de Vries, N. K. (2011). Strategies to facilitate exposure to internet-delivered health behavior change interventions aimed at adolescents or young adults: a systematic review. Health Education \& Behavior: The Official Publication of the Society for Public Health Education, 38(1), 49-62. https://doi. org/10.1177/1090198110372878

Crutzen, R., de Nooijer, J., Candel, M. J. J. M., \& deVries, N. K. (2008). Adolescents who intend to change multiple health behaviors choose greater exposure to an internet-delivered intervention. Journal of Health Psychology, 13(7), 906-11. https://doi.org/10.1177/1359105308095064

Crutzen, R., Ruiter, R. a C., \& de Vries, N. K. (2014). Can interest and enjoyment help to increase use of Internet-delivered interventions? Psychology \& Health, 29(June 2014), 1-18. https://doi.or $\mathrm{g} / 10.1080 / 08870446.2014 .921300$

de Graaf, H., Meijer, S., Poelman, J., \& Vanwesenbeeck, I. (2005). Seks onder je 25e. Definitieve resultaten. Rutgers Nisso Groep/ Soa Aids Nederland. Retrieved from http://www.edudivers. $\mathrm{nl} /$ doc/onderzoek/rapport Seks onder je 25e.pdf

Dobson, D., \& Cook, T. J. (1980). Avoiding type 3 errors in program evaluation: results from a field experiment. Evaluation and Program Planning, 3(4), 269-276.

Domitrovich, C., Bradshaw, C., Poduska, J., Hoagwood, K., Buckley, J., Olin. S., Romanelli, L., Leaf, P., Greenberg, M., \& Ialongo, N. (2008). Maximizing the Implementation Quality of EvidenceBased Preventive Interventions in Schools: A Conceptual Framework. Advanced School of Mental Helath Promotion, 1(3), 6-28.

Domitrovich, C. E., \& Greenberg, M. T. (2000). The Study of Implementation: Current Findings From Effective Programs that Prevent Mental Disorders in School-Aged Children. Journal of Educational and Psychological Consultation, 11(2), 193-221. https://doi.org/10.1207/ S1532768XJEPC1102

Durlak, J. A. (2013). The Importance of Quality Implementation for Research, Practice, and Policy. ASPE Research Brief, (February), 1-16.

Durlak, J. A. (1998). Why program implementation is important. Journal of Presention \& Intervention in the Community, 17(2), 5-18. https://doi.org/10.1300/J005v17n02

Durlak, J. A., \& DuPre, E. P. (2008). Implementation matters: A review of research on the influence of implementation on program outcomes and the factors affecting implementation. American Journal of Community Psychology, 41(3-4), 327-350. https://doi.org/10.1007/s10464-0089165-0 
Dusenbury, L., Brannigan, R., Falco, M., \& Hansen, W. B. (2003). A review of research on fidelity of implementation: Implications for drug abuse prevention in school settings. Health Educ Res, 18(2), 237-256. https://doi.org/10.1093/her/18.2.237

Dusenbury, L., Brannigan, R., Hansen, W. B., Walsh, J., \& Falco, M. (2005). Quality of implementation: Developing measures crucial to understanding the diffusion of preventive interventions. Health Education Research, 20(3), 308-313. https://doi.org/10.1093/her/cyg134

Ertmer, P., \& Ottenreit-Leftwich, A. (2010). Teacher Technology Change: How Knowledge, Confidence, Beliefs, and Culture Intersect. Teacher Technology Change: How Knowledge, Confidence, Beliefs, and Culture Intersect, 42(3), 255-284.

Fagan, A. A., \& Mihalic, S. (2003). Strategies for enhancing the adoption of school-based prevention programs: Lessons learned from the blueprints for violence prevention replications of the life skills training program. Journal of Community Psychology, 31(3), 235-253. https://doi. org/10.1002/jcop.10045

Farmer-Dougan, V., Viechtbauer, W., \& French, T. (1999). Peer-prompted social skills: The role of teacher consultation in student success. Educational Psychology, 19(2), 207-219. https://doi. org/http://dx.doi.org/10.1080/0144341990190207

Fielden, S. (2005). Literature review: coaching effectiveness - a summary. NHS Leadership Centre. Retrieved from: http://literacy.kent.edu/coaching/information/Research/NHS CDWPCoachingEffectiveness.pdf

Fisher, J., \& Fisher, W. (1992). Changing AIDS risk behavior. Psychological Bulletin, 111, 455-474.

Fixens, D.L., Naoom, S.F., Blase, K.A., Friedman, R.M., \& Wallace, F. (2005). Implementation research: A synthesis of the literature. Florida. Retrieved from http://nirn.fpg.unc.edu/sites/nirn.fpg.unc. edu/files/resources/NIRN-MonographFull-01-2005.pdf

Flay, B. R., Hansen, W. B., Johnson, C. A., Collins, L. M., Dent, C. W., Dwyer, K. M., Grossman, L., Hockstein, G., Rauch, J., Sobel, J. L., Sobol, D. F., Sussman, A., \& Ulene, A. (1987). Implementation effectiveness trial of a social influences smoking prevention program using schools and television. Health Education Research, 2(4), 385-400. https://doi.org/10.1093/her/2.4.385

Fleuren, M., Wiefferink, K., \& Paulussen, T. (2004). Determinants of innovation within health care organizations: literature review and Delphi study. Int J Qual Health Care, 16(2), 107-123. https://doi.org/10.1093/intqhc/mzh030

Forman, S. G., Olin, S. S., Hoagwood, K. E., Crowe, M., \& Saka, N. (2009). Evidence-Based Interventions in Schools: Developers' Views of Implementation Barriers and Facilitators. School Mental Health, 1(1), 26-36. https://doi.org/10.1007/s12310-008-9002-5

Fulpen, M van., Bakker, F., Breeman, I., Poelman, J., Schaalma, H., \& Vanwesenbeeck, I. (2002). Seksualiteit en seksuele vorming: een effectonderzoek naar de vernieuwde versie van het lespakket LLL. Utrecht. 
Giling, A., \& Laan, N. Van Der. (2005). Onderzoek naar ICT-gebruik onder docenten in het primair en voortgezet onderwijs/Study into ICT-use among teachers in primary and secondary education. TNS NIPPO, Amsterdam. Retrieved from: http://downloads.kennisnet.nl/ onderzoek/onderzoektnsnipoonderdocenten2005.pdf

Glasgow, R. E., Vogt, T. M., \& Boles, S. M. (1999). Evaluating the public health impact of health promotion interventions: the RE-AIM framework. American Journal of Public Health, 89(9), 1322-1327.

Glasgow, R. E., Vinson, C., Chambers, D., Khoury, M. J., Kaplan, R. M., \& Hunter, C. (2012). National institutes of health approaches to dissemination and implementation science: Current and future directions. American Journal of Public Health, 102(7), 1274-1281. https://doi. org/10.2105/AJPH.2012.300755

Gottfredson, D. C., \& Gottfredson, G. D. (2002). Quality of School-Based Prevention Programs : Results From a National Survey. Journal of Research on Crime and Delinquency, 39(1), 3-35.

Green, L., \& Kreuter, M. (1999). Health promotion planning: An educational and ecological approach. Mountain View: Mayfield.

Green, L.W., \& Kreuter, M. W. (2005). Health Program Planning: An Educational and Ecological Approach (Fourth). New York: McGraw-Hill.

Greenberg, M. (2004). Current and future challenges in school-based prevention: The researcher perspective. Prevention Science, 5, 5-13.

Grube, J.W., Morgan, M., \& McGree, S. (1986). Attitudes and normative beliefs as predictors of smoking intentions and behavior: a test of three models. British Journal of Social Psychology, 25, 81-93.

Hall, G.E., Dirksen, D.J., \& George, A. (2006). Measuring implementation in schools: Levels of use. Austin: SEDL.

Han, S. S., \& Weiss, B. (2005). Sustainability of teacher implementation of school-based mental health programs. Journal of Abnormal Child Psychology, 33(6), 665-679. https://doi. org/10.1007/s10802-005-7646-2

Hansen, W. B., Bishop, D. C., \& Bryant, K. S. (2009). Using online components to facilitate program implementation: Impact of technological enhancements to all stars on ease and quality of program delivery. Prevention Science, 10(1), 66-75. https://doi.org/10.1007/s11121-0080118-5

Hargreaves, H., \& Fink, D. (2006). Sustainable Leadership. San Fransisco: Jossey-Bass Publishers.

Hesselink, A., van der Sluis, M., \& Martens, M. (2011). Implementatieonderzoek Lekker fit! Amsterdam. Retrieved from http://www.rescon.nl/wp-content/uploads//2011/10/10.13.-NHS.-Lekkerfit-management-summary-OP-WEBSITE.pdf

Hoekstra, F., Beek, J ter., Wiefferink, C.H., \& Buijs, G. (2007). Proefimplementatie van het PRIMA antipestbeleid op basisscholen. Tijdschrift van Gezondheidswetenschappen, 85, 195-198. 
Hoekstra, F., Velderman, M. K., Wiefferink, K., Overveld, C. Van, Beek, J., Buijs, G., Goosen, F., \& Paulussen, T. (2009). De adoptiefase van schoolgerichte preventieprogramma's. Van boodschap naar beslissing. Tijdschrift van Gezondheidswetenschappen, (1), 27-36.

Hoelscher, D. M., Evans, A., Parcel, G. S., \& Kelder, S. H. (2002). Designing effective nutrition interventions for adolescents. Journal of the American Dietetic Association, 102, 52-63.

Hofstetter, H., Peters, L., Meijer, S., van Keulen, H., Schutte, L., \& van Empelen, P. (2014). Evaluation of the effectiveness and implementation of the sexual health program Long Live Love iv. European Health Psychologist, 16, 489.

Humphrey, N., Lendrum, A., \& Wigelsworth, M. (2010). Social and emotional aspects of learning (SEAL) program in secondary schools: National evaluation. Manchester.

Hunter, L., Elias, M. J., \& Norris, J. (2001). School based violence prevention: Challenges and lessons learned from an action research project. Journal of School Psychology, 39(2), 161 175. Retrieved from https://login.ezproxy.net.ucf.edu/login?auth=shibb\&url=http://search. ebscohost.com/login.aspx?direct=true \&db=psyh\&AN=2001-17684-003\&site=ehost-live

Jemmott, J., \& Jemmott, L. (2000). HIV reduction behavioral interventions with heterosexual adolescents. AIDS, 14, 40-52.

Johnson, C. C., Li, D., Galati, T., Pedersen, S., Smyth, M., \& Parcel, G. S. (2003). Maintenance of the classroom health education curricula: results from the CATCH-ON study. Health Education \& Behavior: The Official Publication of the Society for Public Health Education, 30(4), 476-88. https://doi.org/10.1177/1090198103253610

Jones, K. M., Wickstrom, K. F., \& Friman, P. C. (1997). The effects of observational feedback on treatment integrity in school-based behavioral consultation. School Psychology Quarterly, 12(4), 316-326. https://doi.org/10.1037/h0088965

Kam CM, Greenberg MT, \& Walls CT. (2003). Examining the role of implementation quality in school-based. Prevention Science, 4(1), 55-63.

Kassenaar, P., \& van Rijswijk, O. (2003). Handboek website usability (First). Academic Service.

Kirby, D., Obasi, A., \& Laris, D. (2006). The Effectiveness of Sex Education and HIV Interventions in Schools in Developing Countries, Preventing HIV/AIDS in Young People: A Systemic Review of the Evidence from Developing Countries. Geneva.

Kirby, D. (2001). Understanding what works and what doesn't work in reducing adolescent sexual risk-taking. Family Planning Perspectives, 33, 276-281.

Kirby, D. (2002). The impact of schools and school programs upon adolescent sexual behavior. Journal of Sex Research, 39(1), 27-33. https://doi.org/10.1080/00224490209552116

Kirby, D. B., Laris, B. A., \& Rolleri, L. A. (2007). Sex and HIV Education Programs: Their Impact on Sexual Behaviors of Young People Throughout the World. Journal of Adolescent Health, 40(3), 206-217. https://doi.org/10.1016/j.jadohealth.2006.11.143 
Kocken, P., Weber, S., Bekkema, N., van Dorst, A., van Kesteren, N., \& Wiefferink, K. (2007). Een inventarisatie van seksuele gezondheidsbevorderingsinterventies voor de jeugd van 10 tot 21 jaar. Leiden. Retrieved from file:///C:/Users/Liz/Downloads/kocken-2007-inventarisatie.pdf

Kohl, L., Crutzen, R., de Vries, N. (2013). Online Prevention Aimed at Lifestyle Behaviors: A Systematic Review of Reviews. Journal of Medical Internet Research, 15(7), e146.

Kok, G., Schaalma, H.P., Ruiter, R.A.C., Brug, J., \& van Empelen, P. (2004). Intervention Mapping: A protocol for applying health psychology theory to prevention programs. Journal of Health Psychology, 9, 85-98.

Kramer, L., Laumann, G., \& Brunson, L. (2000). Implementation and diffusion of the Rainbows program in rural communities: Implications for school-based prevention programming. Jouranl of Ediucational and Psychological Consultation, 11(1), 37-64.

Lane, K., Mahdavi, J., \& Borthwick-Duffy, S. (2003). Teacher perceptions of the prereferral intervention process: A call for assisatance with school-based interventions. Preventing School Failure: Alternative Education for Children and Youth, 47(4), 148-155.

Lendrum, A. G. (2010). Implementing social and emotional aspects of learning (SEAL) in secondary schools in England: Issues and implications. University of Manchester.

Lendrum, A., \& Humphrey, N. (2012). The importance of studying the implementation of interventions in school settings. Oxford Review of Education, 38(5), 635-652. https://doi.org/ 10.1080/03054985.2012.734800

Levenson-Gingiss, Phyllis., \& Hamilton, R. (1989). Determinants of teachers' plans to continue teaching a sexuality education course. Family and Community Health, 13(3), 40-53.

Lillehoj, C., Griffin K., \& Spoth, R. (2004). Program provider and observer ratings of school-based preventive intervention implementation: Agreement and relation to youth outcomes. Health Education \& Behavior, 31(2), 242-257.

Little, M.A., Riggs, N.R., Shin, H., Tate, E.B., \& Pentz, M. (2015). The effect of teacher fidelity of implementation of Pathways to Health on student outcomes. Evaluation \& the Health Professions, 38(1), 21-41. https://doi.org/10.1177/0163278713489879

Makai, P., Perry, M., Robben, S. H. M., Schers, H. J., Heinen, M. M., Rikkert, M. G. M. O., \& Melis, R. F. (2014). Evaluation of an ehealth intervention in chronic care for frail older people: Why adherence is the first target. Journal of Medical Internet Research, 16(6), 1-8. https://doi. org/10.2196/jmir.3057

Marino, G. (2004). E-coaching: Connecting learners to solutions. In The Performance Improvement Emporium (PIE) (Second). San Diego: San Diego State University.

Martens, M., Van Assema, P., Paulussen, T., Schaalma, H., \& Brug, J. (2006). Krachtvoer: Process evaluation of a Dutch program for lower vocational schools to promote healthful diet. Health Education Research, 21(5), 695-704. https://doi.org/10.1093/her/cyl082

McAlister, A. (1995). Behavioral journalism: Beyond the marketing model for health communication. American Journal of Health Promotion, 9(6), 417-420. https://doi.org/10.4278/0890-11719.6.417 
McCormick, L.K., Steckler, A.B., \& McLeroy, K. (1995). Diffusion of innovations in schools: a study of adoption and implementation of school-based tobacco prevention curricula. American Journal of Health Promotion, 9(3), 210-219.

Mihalic, S. F., Fagan, A. A., \& Argamaso, S. (2008). Implementing the Life Skills Training drug prevention program: factors related to implementation fidelity. Implementation Science, 3, 5. https://doi.org/10.1186/1748-5908-3-5

Miller-Day, M., Pettigrew, J., Hecht, M., Shin, Y., Graham, J., \& Krieger, J. (2013). How prevention curricula are taught under real-world conditions: Types of and reasons for teacher curriculum adaptations. Health Education, 113(4), 324-344.

Mortenson, B., \& Witt, J. (1998). The use of weekly performance feedback to increase teacher implementation of a prereferral academic intervention. School Psychology Review, 27, 613627.

Nelson, G., Poehler, D., \& Johnson, I. (1988). Implementation of the Teenage Health Teaching Modules: A Case Study. Health Education, 19, 14-18.

Nielsen, J. (1999). Designing Web Usability: The Practice of Simplicity (First). Indianapolis: New Riders.

Nijland, N., Van Gemert-Pijnen, J. E. W. C., Kelders, S. M., Brandenburg, B. J., \& Seydel, E. R. (2011). Factors influencing the use of a web-based application for supporting the self-care of patients with type 2 diabetes: A longitudinal study. Journal of Medical Internet Research, 13(3). https://doi.org/10.2196/jmir.1603

Noell, G. H., Witt, J. C., Gilbertson, D. N., Ranier, D. D., \& Freeland, J. T. (1997). Increasing teacher intervention implementation in general education settings through consultation and performance feedback. School Psychology Quarterly, 12(1), 77-88. https://doi.org/10.1037/ h0088949

Norton, W.E., Amico, K.R., Cornman, D.H., Fisher, W.A., \& Fisher, J. (2009). An agenda for advancing the science of implementation of evidence-based HIV prevention interventions. Aids \& Behavior, 13, 424-429.

Oldenburg, B., \& Parcel, G. (2002). Diffusion of innovations. In Health behavior and health education: Theory, research and practice. San Fransisco: Jossey-Bass.

Oldenburg, B. F., Sallis, J. F., Ffrench, M. L., \& Owen, N. (1999). Health promotion research and the diffusion and institutionalization of interventions. Health Education Research, 14(1), 121-130. https://doi.org/10.1093/her/14.1.121

Parcel, G., Ross, J., Lavin, A. T., Portnoy, B., Nelson, G. D., \& Winters, F. (1991). Enhancing implementation of the teenage health teaching modules. Journal of School Health, 61, 35-8.

Parcel, G. S., Perry, C. L., Kelder, S. H., Elder, J. P., Mitchell, P. D., Lytle, L. A., Johnson, C. C., \& Stone, E. J. (2003). School climate and the institutionalization of the CATCH program. Health Educ Behav, 30(4), 489-502. https://doi.org/10.1177/1090198103253650

Parsloe, E., \& Leedham, M. (2009). Coaching and Mentoring: Practical Conversations to Improve Learning (Second). London: Kogan Page. 
Paulussen, T., Kok, G., Schaalma, H., \& Parcel, G. (1995). Diffusion of AIDS curricula among Dutch secondary school teachers. Health Education Quarterly, 22, 227-243.

Paulussen, T., Wiefferink, K., \& Mesters, I. (2007). Invoering van effectief gebleken interventies. In Gezondheidsvoorlichting en gedragsverandering. Assen: Van Gorcum.

Paulussen, T., \& Wiefferink, C. (2003). Implementatie is geen vanzelfsprekenheid. Jeugd gezondheidsZorg, 4, 68-71.

Paulussen, T. (1994). Adoption and implementation of AIDS education in Dutch secondary schools. Utrecht.

Paulussen, T. G., Kok, G., \& Schaalma, H. P. (1994). Antecedents to adoption of classroom based AIDS education in secondary schools. Health Educ Res, 9(4), 227-243.

Payne, A. A. (2009). Do predictors of the implementation quality of school-based prevention programs differ by program type? Prevention Science, 10(2), 151-167.https://doi.org/10.1007/ s11121-008-0117-6

Payne, A. A., Gottfredson, D. C., \& Gottfredson, G. D. (2006). School predictors of the intensity of implementation of school-based prevention programs: Results from a national study. Prevention Science, 7(2), 225-237. https://doi.org/10.1007/s11121-006-0029-2

Perry-Casler, S. M., Price, J. H., Telljohann, S. K., \& Chesney, B. K. (1997). National assessment of early elementary teachers perceived self-efficacy for teaching tobacco prevention based on the CDC guidelines. The Journal of School Health, 67(8), 348-354.

Perry, C., Murray, D., \& Griffin, G. (1990). Evaluating the statewide dissemination of smoking prevention curricula: factors in teachers' compliance. Journal of School Health, 60, 501-4.

Peters, L. W. H., Kok, G., Ten Dam, G. T. M., Buijs, G. J., \& Paulussen, T. G. W. M. (2009). Effective elements of school health promotion across behavioral domains: a systematic review of reviews. BMC Public Health, 9, 182. https://doi.org/10.1186/1471-2458-9-182

Pijpers, A.G.M., van Montfort, K. \& Heemstra, F. J. (2002). Acceptatie van ict. Theorie en een veldonderzoek onder topmanagers. Bedrijfskunde, 74(4), 76-84.

Plummer, M. L., Wight, D., Obasi, A. I. N., Wamoyi, J., Mshana, G., Todd, J., Mazige, B. C., Makokha, M., Hayes, R. J., \& Ross, D. A. (2007). A process evaluation of a school-based adolescent sexual health intervention in rural Tanzania: The MEMA kwa Vijana program. Health Education Research, 22(4), 500-512. https://doi.org/10.1093/her/cyl103

Poelman, J. (2008). Databank effectieve jeugdinterventies: beschrijving "Lang Leve de Liefde 3." Amsterdam.

Rabin, B.A., \& Brownson, R. (2012). Developing the terminology for dissemination and implementation research in health. In Dissemination and Implementation Research in Health: Translating Science to Practice. New York: Oxford University Press. 
Resnicow, K., Davis, M., Smith, M., Lazarus-Yaroch, A., Baranowski, T., Baranowski, J., Doyle, C., \& Wang, D. T. (1998). How best to measure implementation of school health curricula: A comparison of three measures. Health Education Research, 13(2), 239-250. https://doi. org/10.1093/her/13.2.239

Riet, J. V.t, Crutzen, R., \& Vries, D. H. (2010). Investigating predictors of visiting, using, and revisiting an online health-communication program: A longitudinal study. Journal of Medical Internet Research, 12(3), e37. https://doi.org/10.2196/jmir.1345

Ringwalt, C. L., Ennett, S., Johnson, R., Rohrbach, L. A., Simons-Rudolph, A., Vincus, A., \& Thorne, J. (2003). Factors associated with fidelity to substance use prevention curriculum guides in the nation's middle schools. Health Education and Behavior, 30(3), 375-391. https://doi. org/10.1177/1090198103253627

Rogers, E. (1995). Diffusion of innovations. New York: the Free Press.

Rogers, E. (2003). Diffusion of Innovations (Fifth). New York: Free Press.

Rohrbach, L, Graham, J., \& Hansen, W. (1993). Diffusion of a school-based substance abuse prevention program: Predictord of program implementation. Preventive Medicine, 22, $237-$ 260.

Rohrbach, L. A., \& Graham, J. H. W. B. (1993). Diffusion of a school-based substance prevention program: Predictors of program implementation. Preventive Medicine, 22, 237-260.

Rohrbach, L. A., Grana, R., Sussman, S., \& Valente, T. W. (2006). Type II Translation. Evaluation \& the Health Professions, 29(3), 302-333. https://doi.org/10.1177/0163278706290408

Rohrbach, L. A., Gunning, M., Sun, P., \& Sussman, S. (2010). The project Towards No Drug Abuse (TND) dissemination trial: Implementation fidelity and immediate outcomes (Prev Sci, 10.1007/s11121-009-0151-z). Prevention Science, 11(1), 113-113. https://doi.org/10.1007/ s11121-009-0155-8

Ross, J., Luepker, R., Nelson, G., Saavedra, P., \& Hubbard, B. (1991). Teenage health teaching modules: Impact of teacher training on implementation and student outcomes. Journal of School Health, 61, 31-4.

Ruiter, R. A. C., Kessels, L. T. E., Peters, G. J. Y., \& Kok, G. (2014). Sixty years of fear appeal research: current state of the evidence. International Journal of Psychology: Journal International de Psychologie, 49(2), 63-70. https://doi.org/10.1002/ijop.12042

Saito, M. (1998). Sex education in school: preventing unwanted pregnancy in adolescents. International Journal of Gynecology \& Obstetrics, 63, 157-160.

Sarama, S., Clements, D., \& Hery, J. (1998). Network of influences in an implementation of a mathematics curriculum innovation. International Journal of Computers for Mathematical Learning, 3, 113-148.

Schaalma, H., Abraham, C., Gillmore, M., \& Kok, G. (2004). Sex education as health promotion: What does it take? Archives of Sexual Behavior, 33(3), 259-269. 
Schaalma, H., \& Kok, G. (2009). Decoding health education interventions: The times are a-changin'. Psychology \& Health, 24, 5-9.

Schaalma, H., Kok, G., Abraham, C., Hospers, H., Klepp, K., \& Parcel, G. (2002). Hiv education for young people: intervention effectiveness, program development, and future research. Prospects, 32(2), 187-206.

Schaalma, H., Kok, G., Poelman, J., \& Reinders, J. (1994). The development of aids education for Dutch secondary schools: a systematic approach based on research, theories and cooperation. In Social psychology and health: European Perspectives. Aldershot, UK: Avebury.

Schaalma, H. P., Kok, G., Bosker, R. J., Parcel, G. S., Peters, L., Poelman, J., \& Reinders, J. (1996). Planned Development and Evaluation of AIDS/STD Education for Secondary School Students in the Netherlands: Short-Term Effects. Health Education \& Behavior, 23(4), 469-487. https://doi. org/10.1177/109019819602300407

Schutte, L., Mevissen, F. E. F., Meijer, S., Paulussen, T., van Empelen, P., \& Kok, G. (2017). Effect evaluation of a web-based coaching intervention to support implementation of sex education among secondary school teachers: Randomized controlled trial. Journal of Medical Internet Research.

Schutte, L., van den Borne, M., Kok, G., Meijer, S., \& Mevissen, F. E. F. (2016). Innovatively supporting teachers' implementation of school-based sex education: Developing a web-based coaching intervention from problem to solution. Journal of Medical Internet Research, 18(7), e136.

Schutte, L., Meertens, R. M., Mevissen, F. E. F., Schaalma, H., Meijer, S., \& Kok, G. (2014). Long Live Love. the implementation of a school-based sex-education program in the Netherlands. Health Education Research, 29(4), 583-597. https://doi.org/10.1093/her/cyu021

Sears, A., \& Jacko, A. (2003). The human-computer interaction handbook: fundamentals, evolving technologies, and emerging applications. New Jersey: Lawrence Erlbaum Associates, Inc., Publishers.

Shepherd, J., Harden, A., Barnett-Page, E., Kavanagh, J., Picot, J., Frampton, G. K., Cooper, K., Hartwell, D., \& Clegg, A. (2014). Using process data to understand outcomes in sexual health promotion: An example from a review of school-based programs to prevent sexually transmitted infections. Health Education Research, 29(4), 566-582. https://doi.org/10.1093/ her/cyt155

Smylie, M. A. (1988). The Enhancement Function of Staff Development: Organizational and Psychological Antecedents to Individual Teacher Change. American Educational Research Journal, 25(1), 1-30. https://doi.org/10.3102/00028312025001001

Steckler, A., Linnan, L. (2002). Process evaluation for public health interventions and research (First). San Fransisco: Jossey-Bass.

Stephenson, J. M., Strange, V., Forrest, S., Oakley, A., Copas, A., Allen, E., Babiker, A., Black, S., Ali, M., Monteiro, H., \& Johnson, A. M. (2004). Pupil-led sex education in England (RIPPLE study): Cluster-randomised intervention trial. Lancet, 364(9431), 338-346. https://doi.org/10.1016/ S0140-6736(04)16722-6 
Sy, A., \& Glanz, K. (2008). Factors Influencing Teachers' Implementation of an Innovative Tobacco Prevention Curriculum for Multiethnic Youth: Project SPLASH. Journal of School Health, 78(5), 264-273.

Van Bruinessen, I. R., van Weel-Baumgarten, E. M., Snippe, H. W., Gouw, H., Zijlstra, J. M., \& van Dulmen, S. (2014). Active Patient Participation in the Development of an Online Intervention. JMIR Research Protocols, 3(4), e59. https://doi.org/10.2196/resprot.3695

Van de Bongardt, D., Mouthaan, I., \& Bos, H. (2009). Seksuele en relationele vorming in het voortgezet onderwijs. Retrieved from http://www.pedagogiek-online.nl/index.php/pedagogiek/article/ viewFile/372/371

van Lieshout, S., Mevissen, F. E. F., de Waal, E., \& Kok, G. (2016). Long Live Love+. Evaluation of the implementation of an online school-based sex education program in the Netherlands. Health Education Research.

Vanwesenbeeck, I., Bakker, F., Fulpen, M van., Paulussen, T., Poelman, J., \& Schaalma, H. (2003). Seks en seksuele risico's bij vmbo-scholieren anno 2002. Tijdschrift Seksuologie, 27, 30-9.

Vanwesenbeeck, I., Westeneng, J., de Boer, T., Reinders, J., van Zorge, R., Boer, T. De, \& Reinders, J. (2015). Lessons learned from a decade implementing Comprehensive Sexuality Education in resource poor settings: The World Starts With Me. Sex Education, 1811(December), 1-16. https://doi.org/10.1080/14681811.2015.1111203

Visser, A. P., \& van Bilsen, P. (1994). Effectiveness of sex education provided to adolescents. Patient Education and Counseling, 23(3), 147-160. https://doi.org/10.1016/0738-3991(94)90031-0

Voogt, J., Sligte, H. W., van den Beemt, A., van Braak. J., \& Aesaert, K. (2016). E-didactiek: Welke-ictapplicaties-gebruiken-leraren-en-waarom? Amsterdam, Kohnstamm Instituut.

Retrieved from http://www.kohnstamminstituut.uva.nl/rapporten/pdf/ki950.pdf

Voogt, C. V., Poelen, E. A. P., Kleinjan, M., Lemmers, L. A., \& Engels, R. C. (2014). The development of a web-based brief alcohol intervention in reducing heavy drinking among college students: An Intervention Mapping approach. Health Promotion International, 29(4), 669-679. https:// doi.org/10.1093/heapro/dat016

Wiefferink, K., Paulussen, P., Linthorst, M., Poelman, J., \& Vanwesenbeeck, I. (2002). Planmatige invoering van het herziende lesprogramma LLL. Een tussentijds verslag van ervaringen van GGD'en met scholen. GGD Nieuws, 14, 17-22.

Wiefferink, K., Paulussen, T., Linthorst, M., Poelman, J., Vanwesenbeeck, I., \& van Wijngaarden, J. (2002). Planmatige invoering van het herziende lesprogramma LLL. Een tussentijds verslag van ervaringen van GGD'en met scholen. GGD Nieuws, 14, 17-22.

Wiefferink, C. H., Poelman, J., Linthorst, M., Vanwesenbeeck, I., Van Wijngaarden, J. C. M., \& Paulussen, T. G.W. (2005). Outcomes of a systematically designed strategy for the implementation of sex education in Dutch secondary schools. Health Education Research, 20(3), 323-333. https:// doi.org/10.1093/her/cyg120

Wight, D., \& Obasi, A. (2003). Unpacking the "black box": the importance of process data to explain outcomes. In Effective Sexual Health Interventions. Oxford Scholarship Online. 
Wind, M., Bjelland, M., Pérez-Rodrigo, C., te Velde, S. J., Hildonen, C., Bere, E., Klepp, K. I., \& Brug, J. (2008). Appreciation and implementation of a school-based intervention are associated with changes in fruit and vegetable intake in 10- to 13-year old schoolchildren - The Pro Children study. Health Education Research, 23(6), 997-1007. https://doi.org/10.1093/her/cym078

Witt, J., Noell, G., LaFleur, L., \& Mortenson, B. (1997). Teacher Use Of Interventions In General Education Settings: Measurement And Analysis Of The Independent Variable. Journal of Applied Behavior Analysis, 30(4), 693-696. https://doi.org/10.1901/jaba.1997.30-693

World Health Organization. (2009). School health and youth health promotion. Retrieved from http://www.who.int/school_youth_health/en/

Zins, J. E., Elias, M. J., Greenberg, M. T., \& Pruett, M. K. (2000). Promoting quality implementation in prevention programs. Journal of Educational and Psychological Consultation, 11, 173-174.

Zwaneveld, B., \& Rigter, H. (2009). Over drempels naar meer ict-gebruik in het voortgezet onderwijs, 76.

Zwart, R. C., Wubbels, T., Bergen, T. C. M., \& Bolhuis, S. (2007). Experienced teacher learning within the context of reciprocal peer coaching. Teachers and Teaching, 13(2), 165-187. https://doi. org/10.1080/13540600601152520 


\section{Valorization}





\section{VALORIZATION}

The social value and relevance of this dissertation is ultimately to contribute to sexual health promotion of the youth in the Netherlands and internationally. The Netherlands is well known for good quality sex education in schools, and compared to other countries, has lower rates of teen pregnancies and sexually transmitted infections (STI's). The youth in the Netherlands, however, still face numerous challenges when it comes to sexual health, including undesired sexual interactions, sexual violence, unsafe online interactions and unsafe sex resulting in unwanted teen pregnancies and STI's. The resulting physical, mental, financial and social consequences are difficult for the individual and put a strain on their environment and society at large. Prevention is essential, not only for the quality of life of the youth, but also to minimize the costs and social burden for the society, that are associated with these sexual health issues.

In order to bring about behavioral change and ultimately enhance sexual health among youth, not only is a qualitatively sound and effective program required but successful implementation of the program is also necessary to ensure the program is used and reaches the target group to have impact. Schools provide the ideal setting to reach adolescents with health promotion initiatives and are, next to parents, one of the most important sources of information for the youth on sexual health. The reach and implementation of school-based sex education programs in particular, however, is not optimal, partly due to sexuality being considered a challenging subject by most schools. The extent to which a program is delivered (completeness), however, and the degree to which it is implemented as intended by program developers (fidelity), are especially important for program effectiveness. Non-used health programs are not only a waste of money but also make positive results less likely to be achieved.

In this dissertation, the implementation of the school-based sex education program, Long Live Love (LLL), is central, with a special focus on Web-based coaching to promote the implementation quality of LLL by teachers. The research presented in this dissertation provides insight in factors influencing the implementation process of $L L L$, and indicated a need to develop different tools to promote each stage of the implementation process, tailored to determinants of that stage. Interpersonal contact, mass media and online approaches were used to address each stage. The implementation strategy was made relevant for experienced and less experienced teachers as well as for innovators and laggards. The means by which the most innovative component of the implementation strategy, namely Web-based e-coaching, was developed, and the outcomes of the evaluation, provide inspiring insights for online means of bringing about behavioral 
change in teacher implementation. This is especially relevant in a world of increasing digital technologies, bringing exciting opportunities for innovative ways of teaching and learning.

Although this research was conducted in the Dutch context, the means by which the implementation strategy was developed and the lessons learned are relevant for other contexts and countries. This dissertation highlights the importance of a systematic approach to developing an implementation strategy, in close collaboration with those who deliver the program (teachers) and those who support the delivery of the implementation strategy (Municipal Health Service professionals). This dissertation further exemplifies implementation as a dynamic process: continuous changes within organizations and in the environmental context call for continuous adaptations of the implementation strategy and the LLL program itself to actuality, changing circumstances, the needs and desires of program users and implementers and to the intervention context in order to ensure its continuation. Program developers not only need to anticipate for program implementation during program development but also need to intervene actively in the implementation process.

Successful implementation of school-based sex education programs can be achieved by putting different pieces of the puzzle together:

a) A well-developed program as a tool, that is compatible with the working methods and preferences of teachers and students;

b) Support in creating the prerequisite conditions to effectively deliver sex education generally, such as creating a safe classroom environment;

c) Support in delivering the program with completeness and fidelity, in addition to;

d) Considering environmental factors that may influence the implementation process such as school-management support, policies and resources. Although absolute completeness and fidelity of school-based (sex) education programs may seem unrealistic due to frequent and sometimes inevitable adaptations, teachers need to be supported regardless, in delivering programs with as much completeness and fidelity as possible, with guidance in delivering principle program components to ensure program effectiveness is preserved. A balance in accommodating teachers and preserving completeness and fidelity is thus required. 
Long Live Love is a successful, effective, evidence-based program which is well appreciated by teachers and their students. This dissertation encourages continued investment in national and international implementation of LLL. It also calls for the formation of policies and laws that support the integration and structural embedment of sex education in schools and that reflect the importance that society ought to place on the sexual health of our youth.

This implementation strategy is not only relevant for the youth, teachers, schools and Municipal Health Services, but also for policy makers and the Ministries of Health and Education: without systematically developed programs and sustainable implementation, the impact of public health activities will not reach their optimal potential. Nationally and world-wide, the youth have the right to appropriate and thorough sex education. A sexually healthy and empowered youth is a happy one who can perform better in school, with fewer drop-outs and contribute meaningfully to society. Successful implementation of systematically developed qualitatively sound sexual health promotion programs is an important means of prevention and contribution to the positive sexual health of the youth, accompanied by the provision of services, policies, and laws that enable, facilitate, reinforce and promote positive sexual health behaviors in the youth and have positive public health impact. 
Summary 



\section{SUM MARY}

This dissertation is about the development and evaluation of an implementation strategy for the national implementation of the secondary school-based sex education program, 'Long Live Love' (LLL) - 'Lang Leve de Liefde', in the Netherlands. The focus is mostly on the most innovative component of the implementation strategy, namely the Web-based coaching intervention 'Lesgevenindeliefde.nl' or 'Teachinglove.nl' to enhance completeness and fidelity of implementation of LLL.

The success and impact of a health promotion program is not only dependent on the quality and content of the program but also on the quality, and extent of its implementation. Implementation is considered a process consisting of different stages, namely dissemination (i.e. awareness), adoption (i.e. form an intention, uptake, accept a program), implementation (i.e. initial use), and continuation (i.e. continued use). Program implementation is, however, often overlooked, insufficiently considered, not planned for or poorly conducted, resulting in a waste of money and limited public health impact. Implementation is not an automatic process: to ensure a program is used and thus reaches the target group, program developers not only need to anticipate for implementation during program development but also need to intervene actively in the implementation process. Schools provide the ideal setting to reach adolescents with health promotion initiatives, yet the reach and implementation of these programs is not optimal. Especially sexual health education programs are poorly implemented in schools; in addition to being a challenging subject and not being formally established in schools, if these programs do get implemented, they are frequently modified during implementation by omitting certain program components or activities, using supplementary material or not executing the program as prescribed by program developers. Being key figures in the delivery of school-based sex education programs, investing in the professional development of teachers is necessary to ensure successful implementation. Thus far, effort has been directed primarily towards stimulating dissemination and adoption of sexual health education programs and less so on implementation and continuation. Implementation, namely the extent to which a program is delivered (completeness) and the degree to which it is implemented as intended by program developers (fidelity) are, however, essential for program effectiveness.

Long Live Love has existed for over 20 years and is by far the most successful, effective, evidence-based program in the field of school-based sex education in the Netherlands. Implementation of this program was not optimal, despite an existing implementation strategy: certain program activities were being omitted by teachers, the program was not always implemented as prescribed by the developers and teachers tended 
to stop using the program after one or more implementations. There was thus room for improving the quality of the implementation. LLL was outdated and was going to be revised. In order to reach the target group, the development of an implementation strategy for the revised LLL program was required. The economic crisis prevalent at the time, impacted the Municipal Health Services (MHS), who support schools in delivering health promotion programs, including LLL. With their capacity being reduced at the time, along with their limited didactic skills and expertise to be appropriate role models for teachers in teaching skills for adequate implementation, another form of support was needed to compensate for the limitations of the MHS and support teachers in the implementation of LLL with completeness and fidelity.

The focus of this dissertation is mostly on the development and evaluation of the most innovative component of the implementation strategy, namely a Web-based coaching intervention 'Lesgevenindeliefde.nl' or 'Teachinglove.nl'. E-coaching was developed to enhance completeness and fidelity of implementation of LLL as this is especially important for effectiveness when putting an innovation into practice. Instead of solely providing program materials (including a teacher manual) and one-time preimplementation training via the MHS, personal assistance and ongoing consultation were required once the transition was made to real-life implementation of LLL by teachers and to complement the existing implementation strategy.

The implementation strategy was systematically planned for and developed using the Intervention mapping (IM) protocol. It was based on theory and evidence and was developed in close collaboration with secondary school teachers (the target group) and the intermediary health promotion professionals from the MHS, who support teachers in school-based health promotion. In the new implementation strategy, different tools were developed to promote each stage of the implementation process, tailored to determinants of that stage. It included interpersonal contact, multimedia and online approaches, to address each stage of the implementation process.

Each chapter of this dissertation describes the steps taken to systematically develop an implementation strategy to reach secondary school students with the revised LLL and have an impact on their relational and sexual health.

Chapter 2 describes the findings of a quantitative study among secondary school teachers $(\mathrm{N}=130)$ on factors associated with each stage of the implementation process in the school setting regarding the previous LLL sex education program. The results highlighted that implementation of LLL was not optimal and could be improved: approximately one-third of the LLL activities were not implemented and among the 
remaining two-thirds of the activities that were implemented, teachers generally did so with limited integrity; lessons were (slightly) modified. Each stage of the implementation process was found to be influenced by different factors: teacher curriculum-related beliefs were primarily associated with adoption, but also with implementation and continuation. Additionally, implementation completeness and fidelity and continued use of LLL were specifically enhanced by contextual factors, namely teacher training and interactive context variables - school policy, governing body support and student response. The factors identified in this study were taken into consideration when developing the revised LLL program, in anticipation of implementation. They also provided insight into the factors that needed to be addressed in the implementation strategy.

Chapter 3 provided insight in the barriers and facilitating factors, influencing the current and potential future supportive role of the MHS in the implementation process of LLL, as perceived by MHS professionals at the time of the study. These insights were gathered by means of surveys $(\mathrm{N}=26)$ and interviews $(\mathrm{N}=19)$. Our study revealed that factors that influenced the implementation-promoting activities of the MHS were related to characteristics of the previous LLL program and the role of teachers, the school, the MHS and the municipality. These factors are thus found on multiple levels, requiring not only a revised LLL program but a multi-level approach to facilitate implementation. This meant a new implementation strategy aimed at supporting the professional development of teachers, convincing schools and municipalities of the

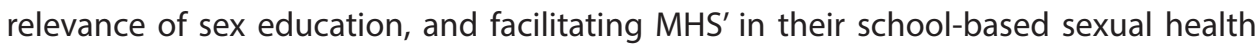
promotion activities. Despite the willingness of MHS professionals, the impact of the 2008 economic crisis limited the role of the MHS to stimulating the dissemination and adoption of the revised LLL. While the MHS remains an important and competent party in the implementation of school-based sexual health education programs, this paper concludes that their restricted capacity, limited didactic skills and the need for ongoing support during real-life implementation necessitates an alternative form of support for teachers during implementation for completeness and fidelity, possibly in the form of online coaching.

Chapter 4 proceeds with a description of the systematic development of the e-coaching website, 'lesgevenindeliefde.nl' or 'teachinglove.nl' as a specific component of the general implementation strategy, using the intervention mapping protocol. During the development process, a discrepancy was revealed between actual need for coaching, according to program developers and teachers own perceived need for coaching in delivering LLL: Despite their implementation behavior, namely completeness and fidelity, being suboptimal according to program developers, teachers themselves did 
not perceive their teaching behavior as problematic and felt resistance to coaching or behavioral change. E-coaching was developed as an efficient, low-threshold way of reaching a mass of teachers in order to support them in the implementation of LLL with completeness and fidelity and to support them in adequately dealing with the difficult situations encountered when delivering SRH lessons, which could lead to improved program implementation. A by-and-for teacher approach was used to create a coaching website which consisted of a self-reflection tool which was used to create awareness of their need for coaching or behavioral change, and support in dealing adequately with difficult situations encountered when delivering SHR lessons through role model stories, video's, tips, background information, and FAQ's. The website also included student materials and a downloadable and printable teacher manual with additional information and suggestions on the LLL program to further enhance the delivery of LLL with completeness and fidelity.

Chapter 5: A cluster randomized controlled trial (e-coaching vs waiting list control) was conducted with a baseline assessment (T0) and follow up (T1) two weeks after completing the LLL program to evaluate the effects of e-coaching on teacher implementation behavior (completeness and fidelity) and its determinants. A total of 43 schools with 83 teachers participated in the study. In the follow-up 38 schools participated; 23 in the e-coaching condition with 41 teachers, 15 in the control condition with 26 teachers. E-coaching was not found to be effective in enhancing completeness and fidelity of LLL by teachers, neither on positively influencing any of the determinants of successful implementation. The lack of effect was attributed to the intervention content, the limited use, and/or the study design itself.

Chapter 6: A process evaluation was conducted, including surveys $(N=67)$ and additional interviews $(\mathrm{N}=20)$ among teachers who had access to the e-coach, in which appreciation and (motives for) use of the e-coach were investigated. Limited use of e-coaching is likely the main reason why it did not have impact, resulting mainly from a low personal relevance, in addition to lack of time and the introduction of two innovations at once- LLL and e-coach. Although Lesgeveindeliefde.nl was perceived by teachers as usable and as an instrumental addition to the LLL program, its personal relevance was rated low. The lack of personal relevance of the e-coach resulted largely from teachers not feeling a need for coaching, thereby not using the website or discontinuing use altogether. This lack of need for coaching was already established in the needs assessment phase of the e-coach development. The e-coach seemed unable to change teachers' perceived need for coaching. Consequently, although the e-coaching intervention contains elements to create the prerequired conditions for completeness and fidelity by focusing predominantly on dealing with difficult situations, 
in its current form, the e-coach may not have been ideal due to insufficiently addressing the importance of completeness and fidelity and not addressing direct, practical means of achieving completeness and fidelity of LLL specifically.

In hindsight, we realize, as a result of this process evaluation, that a discrepancy exists between teachers and e-coach developers on the perceived need for coaching which is related to a different interpretation regarding quality of teaching. For teachers, 'correct' program delivery and thus good teaching is related to having sufficient didactic skills. For program developers, however, 'correct' program delivery is related to implementation with completeness and fidelity. It seems teachers may first have to understand the importance of completeness and fidelity in the implementation of LLL and consider it a component of good teaching, prior to behavioral change through e-coaching. The ecoaching intervention itself will need to be optimized to address the importance of completeness and fidelity, and contain more tools aimed at enhancing the completeness and fidelity of LLL specifically. Use of the e-coach needs to be stimulated by increasing the personal relevance of the website, and utilizing the broader implementation strategy.

Chapter 7: The dissertation ends with a general discussion, including implications and recommendations for research and the public health field.

Guiding the implementation process and changing implementation behavior of intermediaries such as teachers and MHS professionals is not a simple task or automatic process. It requires anticipation for implementation during program development, actual systematic development of different theory- and evidence-based strategies at the individual and environmental level to promote each step of the implementation process and continued investment in professional development. Intervention Mapping is a useful tool to ensure timely planning and bottom-up development of an implementation strategy.

Achieving completeness and fidelity of SRH program delivery is a puzzle composed of several components. In the case of LLL, more than just e-coaching is required. Although e-coaching itself needs to be improved and its use stimulated, it is only one component of a broader implementation strategy that is needed to support the entire implementation process of LLL. The likelihood of achieving completeness and fidelity of LLL can be increased by: 
a) Realization that the target group could be operating from a different reference framework and creating a mutual understanding of 'correct' program delivery between program developers and teachers;

b) Awareness among teachers of the importance of completeness and fidelity; that it should be done and why it is important and that it is also a component of teaching well;

c) A well-developed program as a tool, in this case LLL, that is compatible with the working methods and preferences of teachers and students;

d) Support in creating the prerequisite conditions to effectively deliver SRH generally, such as how to create a safe environment in the classroom to discuss sexuality;

e) Support in delivering LLL specifically with completeness and fidelity;

f) A broader implementation strategy to stimulate the implementation process of LLL.

Despite these efforts, in real life, absolute completeness and fidelity of school-based (sex) education programs seems unrealistic: Adaptations often occur and are sometimes inevitable. Environmental factors outside the control of teachers can also influence the degree to which a program is implemented and need to be taken into consideration. Teachers should therefore be supported in delivering programs with as much completeness and fidelity as possible, with guidance in delivering principle program components to ensure program effectiveness is preserved. This requires creativity from program developers to accommodate teachers as well as preserve completeness and fidelity as much as possible.

Continuous changes within organizations and in the environmental context call for continuous adaptation of the implementation strategy and the LLL program itself to actuality, changing circumstances, the needs and desires of program users and implementers and to the intervention context in order to ensure its continuation. This exemplifies implementation as a dynamic process. A successful implementation of LLL should ultimately lead to young people being better prepared, motivated and able to practice safe sex and have mutual, pleasant relations. 

Samenvatting 



\section{SAMEN VATTING}

Dit proefschrift beschrijft de ontwikkeling en evaluatie van een implementatiestrategie voor de nationale implementatie van het lesprogramma 'Lang Leve de Liefde' (LLL) voor de onderbouw van het voortgezet onderwijs in Nederland. In dit proefschrift ligt de focus vooral op het meest innovatieve onderdeel van de implementatiestrategie, namelijk de web-based coaching interventie 'Lesgevenindeliefde.nl' die is ontwikkeld om de volledigheid en getrouwheid bij de implementatie van LLL door docenten te verbeteren.

Het succes en de impact van een gezondheidsbevorderingsprogramma is niet alleen afhankelijk van de kwaliteit en de inhoud van het programma zelf, maar ook van de kwaliteit en de volledigheid waarmee het programma is geïmplementeerd. Implementatie wordt beschouwd als een proces dat bestaat uit verschillende fasen, namelijk verspreiding (d.w.z. bewustmaking), adoptie (d.w.z. een intentie, opname, een programma accepteren), implementatie (d.w.z. initieel gebruik) en continuïteit (d.w.z. voortgezet gebruik). Echter, de mate waarin programma's inderdaad goed worden geïmplementeerd krijgt vaak zowel in onderzoek als praktijk te weinig aandacht, wordt onvoldoende overwogen, niet gepland of slecht uitgevoerd, wat resulteert in een verspilling van geld en beperkte impact voor de volksgezondheid. Implementatie is niet een automatisch proces: om ervoor te zorgen dat een programma wordt gebruikt en dus de doelgroep bereikt, moeten ontwikkelaars niet alleen anticiperen op implementatie tijdens programma-ontwikkeling, maar ook actief ingrijpen in het implementatieproces. Scholen bieden de ideale setting om adolescenten te bereiken met initiatieven voor gezondheidsbevordering, maar de verspreiding en de implementatie van deze programma's is meestal niet optimaal. Vooral seksuele gezondheidsprogramma's worden vaak slecht geïmplementeerd in scholen. Naast het feit dat seksualiteit een lastig onderwerp kan zijn en het bij aanvang van het in dit proefschrift beschreven project ook nog geen onderwerp was dat formeel was vastgelegd in de leerdoelen van middelbare scholen, is het ook een onderwerp waarbij docenten vaak bij de aan hen beschikbaar gestelde programma's bepaalde programmacomponenten of -activiteiten weg laten, aanvullende (vaak zelf ontwikkelde) materialen gebruiken of het programma niet uit voeren zoals voorgeschreven door de ontwikkelaars van het programma. Als sleutelfiguren bij de uitvoering van seksuele gezondheidsprogramma's op school, is het nodig om te investeren in de deskundigheidsbevordering van docenten om succesvolle implementatie te waarborgen. Tot nu toe is er vooral gestreefd naar het stimuleren van verspreiding en adoptie van seksuele gezondheidsprogramma's en was er minder aandacht voor implementatie en continuïteit. Implementatie - de mate waarin een 
programma wordt uitgevoerd (volledigheid/completeness) en de mate waarin het wordt geïmplementeerd zoals bedoeld door programmaontwikkelaars (getrouwheid/ fidelity) - is echter essentieel voor programma-effectiviteit.

Lang Leve de Liefde bestaat al meer dan 20 jaar en is veruit het meest succesvolle, effectieve, op evidentie gebaseerde programma op het gebied van seksuele en relationele vorming op scholen in Nederland. Uit onderzoek bleek dat de implementatie van dit programma niet optimaal was, ondanks een bestaande implementatiestrategie; bepaalde programma-activiteiten werden door docenten weggelaten. Het programma werd niet altijd geïmplementeerd zoals voorgeschreven door de ontwikkelaars en docenten waren geneigd te stoppen met gebruik van het programma na het één of enkele keren te hebben gebruikt. De kwaliteit van de implementatie kon dus verbeterd worden. Bovendien was LLL verouderd en moest deze worden herzien. Om de doelgroep optimaal te bereiken, en de volledigheid en getrouwheid van implementatie te bevorderen, was voor het herziene LLL-programma de ontwikkeling van een implementatiestrategie nodig. De economische crisis ten tijde van de start van het in dit proefschrift beschreven project (2011), heeft invloed gehad op de implementatiebijdrage van gemeentelijke gezondheidsdiensten (GGD), die scholen normaliter ondersteunden bij het uitvoeren van gezondheidsbevorderende programma's, zoals LLL. Hun capaciteit was door de crisis beperkt. Daarnaast bleek dat er onder GGD medewerkers een gebrek aan didactische vaardigheden en deskundigheid was om voor docenten passende rolmodellen te zijn om implementatie vaardigheden aan te leren voor een adequate implementatie. Een andere vorm van ondersteuning bleek dus nodig om docenten te ondersteunen bij de implementatie van LLL en daarmee te compenseren voor de beperkingen van de GGD.

De focus van dit proefschrift is vooral op de ontwikkeling en evaluatie van het meest innovatieve onderdeel van de implementatiestrategie, namelijk een webbased coaching interventie 'Lesgevenindeliefde.nl'. E-coaching is ontwikkeld om de volledigheid en getrouwheid van de implementatie van LLL te verbeteren, omdat die belangrijk zijn voor de effectiviteit van een innovatie. In plaats van het uitsluitend verstrekken van programmamateriaal (inclusief een docenthandleiding) en een eenmalige pre-implementatie training voor docenten via de GGD, is daarnaast - ter aanvulling op de bestaande implementatiestrategie - doorlopende persoonlijke begeleiding en ondersteuning nodig zodra de overgang is gemaakt naar de werkelijke implementatie van LLL door docenten. 
De (deels in dit proefschrift beschreven) implementatie strategie voor LLL is systematisch gepland en ontwikkeld met behulp van het Intervention Mapping (IM) protocol. De strategie is daarmee gebaseerd op theorie en evidentie en werd ontwikkeld in nauwe samenwerking met docenten in het voortgezet onderwijs (de doelgroep) en de intermediaire professionals van de GGD. Voor deze nieuwe implementatiestrategie werden verschillende instrumenten ontwikkeld om elke fase van het implementatieproces positief te beïnvloeden, afgestemd op de determinanten van die fase. De implementatiestrategie omvat interpersoonlijk contact, multimedia en online benaderingen om elke fase van het implementatieproces aan te pakken.

Elk hoofdstuk van dit proefschrift beschrijft de stappen die zijn genomen om systematisch een implementatiestrategie te ontwikkelen, daarmee leerlingen in het voortgezet onderwijs te bereiken met de herziene LLL, en een positieve invloed te hebben op hun relationele en seksuele gezondheid.

Hoofdstuk 2 beschrijft de bevindingen van een kwantitatieve studie onder docenten in de onderbouw van het voortgezet onderwijs $(N=130)$ naar factoren die geassocieerd zijn met elk stadium van het implementatieproces met betrekking tot het vorige LLL programma over relaties en seksualiteit. Uit de resultaten blijkt dat de implementatie van LLL niet optimaal was en verbeterd kon worden: ongeveer een derde van de LLL-activiteiten werden niet geïmplementeerd. Met betrekking tot de overige tweederde van de activiteiten die wel werden geïmplementeerd, gebeurde dit niet altijd zoals beschreven in de handleiding; lessen werden (licht) gewijzigd. Elke fase van het implementatieproces bleek beïnvloed te worden door verschillende factoren. De zogenaamde outcome beliefs (dat wil zeggen de verachtingen die er zijn t.a.v. de uitkomsten van het programma) van docenten waren vooral belangrijk voor adoptie, en in mindere mate voor implementatie en continuïteit.

Daarnaast werden de volledigheid en getrouwheid van implementatie, als ook de continuïteit van LLL, specifiek verbeterd door contextuele factoren, zoals docenten trainingen, schoolbeleid, steun vanuit het bestuur en leerlingenrespons. De in deze studie geïdentificeerde factoren werden in aanmerking genomen bij het ontwikkelen van het herziene LLL-programma. Het onderzoek heeft ook inzicht gegeven in de factoren die in de implementatiestrategie zouden moeten worden aangepakt.

Hoofdstuk 3 heeft inzicht gegeven in de belemmerende en faciliterende factoren die van invloed zijn op de (toekomstige) ondersteunende rol van de GGD bij het implementatieproces van LLL. Deze inzichten werden verzameld door middel van vragenlijsten $(\mathrm{N}=26)$ en interviews $(\mathrm{N}=19)$. Uit onze studie bleek dat factoren die de 
(implementatie bevorderende) activiteiten van de GGD beïnvloeden verband houden met enerzijds de kenmerken van het toenmalige LLL-programma en anderzijds met de rol van docenten, de school, de GGD en de gemeente. Deze factoren worden dus op meerdere niveaus gevonden, waarbij voor het bevorderen van implementatie niet alleen een herziende LLL-programma nodig is, maar ook een aanpak op al deze niveaus. Deze resultaten toonde het belang aan van een nieuwe implementatiestrategie gericht op deskundigheidsbevordering van docenten, het overtuigen van scholen en gemeenten van de relevantie van relationele en seksuele vorming en het faciliteren van GGD-en in hun implementatie-bevorderende activiteiten op school. Ondanks de bereidheid van GGD-professionals, heeft de economische crisis, die begon in 2008, de rol van de GGD beperkt tot het stimuleren van de verspreiding en adoptie van het herziene LLL programma. Hoewel de GGD een belangrijke en competente partij blijft bij de uitvoering van gezondheidsbevorderende programma's voor scholen, inclusief seksuele gezondheid, concluderen we in deze studie dat hun beperkte capaciteit, beperkte didactische vaardigheden en de noodzaak van een continue docentondersteuning tijdens implementatie vraagt om een alternatieve vorm van steun aan docenten tijdens de implementatie van LLL. Dit kan mogelijk in de vorm van online coaching.

Hoofdstuk 4 gaat verder met een beschrijving van de systematische ontwikkeling van de e-coaching website, 'lesgevenindeliefde.nl' als een specifiek onderdeel van de algemene implementatiestrategie, met behulp van het Intervention Mapping (IM) protocol. Tijdens het ontwikkelingsproces bleek er een discrepantie te zijn tussen de daadwerkelijke behoefte aan coaching volgens de ontwikkelaars en de waargenomen behoefte aan coaching volgens docenten, bij het uitvoeren van LLL: ondanks dat hun implementatiegedrag (d.w.z. volledigheid en getrouwheid) suboptimaal was volgens de programma-ontwikkelaars, beschouwden docenten hun manier van lesgeven niet als problematisch en toonde ze weerstand tegen coaching of gedragsverandering. E-coaching is daarom ontwikkeld als een efficiënte, laagdrempelige manier om een groot aantal docenten te bereiken en om hen te ondersteunen bij het volledig en getrouw implementeren van LLL. Daarnaast is e-coaching ontwikkeld om docenten te ondersteunen in het omgaan met de lastige situaties die zich voordoen bij het geven van relationele en seksuele vorming wat kan leiden tot een verbeterde uitvoering van deze programma's. Een voor-en-door docenten benadering werd gebruikt om een coaching website te maken die bestaat uit een zelfreflectie tool met als doel om docenten bewust te maken van hun behoefte aan coaching of gedragsverandering, en ondersteuning in het adequaat omgaan met lastige situaties tijdens het lesgeven over relaties en seksualtiet door middel van ervaringsverhalen, video's, tips, achtergrondinformatie 
en FAQ's. De website bevat ook lesmaterialen en een downloadbare en afdrukbare docentenhandleiding met aanvullende informatie en suggesties over het LLL programma om de implementatie van LLL verder te verbeteren.

Hoofdstuk 5: Een cluster gerandomiseerde en gecontroleerde evaluatie (e-coaching versus wachtlijst controle) werd uitgevoerd met een voormeting (T0) en follow-up (T1) twee weken na het afronden van het LLL programma om de effecten van e-coaching op het implementatie gedrag van docenten (volledigheid en getrouwheid) en de determinanten te evalueren. In totaal namen 43 scholen met 83 leraren deel aan de studie. In de follow-up deden 38 scholen mee; 23 in de e-coaching conditie met 41 docenten, 15 in de controle conditie met 26 docenten. E-coaching bleek niet effectief te zijn om de volledigheid en getrouwheid van de implementatie van LLL door docenten te verbeteren, noch bleek het een positieve invloed te hebben op de determinanten van succesvolle implementatie. Het gebrek aan effect werd toegeschreven aan de interventie inhoud, het beperkte gebruik en / of het studie ontwerp zelf.

Hoofdstuk 6: $\mathrm{Er}$ is een procesevaluatie uitgevoerd, door middel van vragenlijsten ( $\mathrm{N}$ $=67)$ en extra interviews $(N=20)$ onder docenten die toegang hadden tot de e-coach, waarin waardering en (motieven voor) gebruik van de e-coach waren onderzocht. Dat e-coaching zo beperkt werd gebruikt, is waarschijnlijk de belangrijkste reden waarom het geen impact heeft gehad; vooral als gevolg van een lage persoonlijke relevantie, naast het gebrek aan tijd en de introductie van twee innovaties tegelijk, LLL en e-coach. Hoewel Lesgeveindeliefde.nl door docenten als bruikbaar en als instrumentele aanvulling op het LLL-programma werd waargenomen, werd de persoonlijke relevantie ervan laag beoordeeld. Het gebrek aan persoonlijke relevantie van de e-coach was vooral het gevolg ervan dat docenten dachten geen coaching nodig te hebben, waardoor de website niet volledig werd gebruikt of het gebruik geheel werd gestaakt. Dit gebrek aan coaching was al in de behoeftenbeoordelingsfase van de e-coachontwikkeling bevestigd. De e-coach leek niet de waargenomen behoefte voor coaching van docenten te veranderen. Dus hoewel de e-coaching, in de huidige vorm, interventie elementen bevat om de vereiste voorwaarden voor volledigheid en getrouwheid te creëren door voornamelijk te focussen op lastige situaties, is de e-coach misschien niet ideaal omdat daarin onvoldoende aandacht wordt besteedt aan het belang van volledigheid en getrouwheid, en het niet gericht is op directe, praktische middelen om die te bereiken.

Achteraf realiseren wij ons als gevolg van deze procesevaluatie dat er sprake is van een discrepantie tussen docenten en e-coach ontwikkelaars op de waargenomen behoefte aan coaching. Een behoefte die gerelateerd is aan een andere interpretatie met betrekking tot de kwaliteit van lesgeven. Voor docenten is het 'correct' uitvoeren 
van een programma en dus goed lesgeven gerelateerd aan voldoende didactische vaardigheden. Voor programma-ontwikkelaars is 'correct' programma-bezorging echter gerelateerd aan implementatie met volledigheid en getrouwheid. Het lijkt erop dat docenten het belang van volledigheid en getrouwheid in de implementatie van LLL eerst moeten begrijpen en het als onderdeel van goed lesgeven moeten beschouwen, voorafgaand aan gedragsverandering door middel van e-coaching. De e-coaching interventie zelf moet worden geoptimaliseerd en zou meer tools moeten bevatten om de volledigheid en getrouwheid van de implementatie van LLL specifiek te verbeteren. Het gebruik van de e-coach moet gestimuleerd worden door de persoonlijke relevantie van de website te vergroten en een bredere implementatiestrategie te gebruiken.

Hoofdstuk 7: Dit proefschrift eindigt met een algemene discussie, inclusief implicaties en aanbevelingen voor onderzoek en het vakgebied.

Het begeleiden van het implementatieproces en het veranderen van implementatiegedrag van intermediairs, zoals docenten en GGD professionals, is geen eenvoudige taak of automatisch proces. Het vereist anticipatie op implementatie tijdens programma ontwikkeling, de feitelijke systematische ontwikkeling van verschillende theorie- en evidence-based strategieën op individueel en omgevingsniveau om elke stap van het implementatieproces te bevorderen, en de voortdurende investeringen in deskundigheidsbevordering. Intervention Mapping is een nuttig instrument om tijdige planning en bottom-up ontwikkeling van een implementatiestrategie te verzekeren.

Het bereiken van optimale volledigheid en getrouwheid van seksueel gezondheidsbevorderende programma's is een puzzel samengesteld uit verschillende componenten. In het geval van LLL is meer dan e-coaching vereist. Hoewel e-coaching zelf verbeterd moet worden en het gebruik ervan ook gestimuleerd, is het slechts één onderdeel van een bredere implementatiestrategie die nodig is om het volledige implementatieproces van LLL te ondersteunen. Dekans om volledigheid en getrouwheid van de implementatie van LLL te bereiken kan worden verhoogd door:

a) Realisatie dat de doelgroep vanuit een ander referentiekader kan werken en een wederzijds begrip creëren voor correcte programmaoverdracht tussen programmaontwikkelaars en docenten;

b) Bewustwording onder docenten van het belang van volledigheid en getrouwheid; dat het moet worden gedaan, waarom het belangrijk is, en dat het een belangrijk onderdeel is van goed lesgeven; 
c) Een goed ontwikkeld programma als een hulpmiddel, in dit geval LLL, dat overeenkomt met de werkwijzen en voorkeuren van docenten en leerlingen;

d) Ondersteuning bij het creëren van de randvoorwaarden voor het effectief uitvoeren van relationele en seksuele vorming in het algemeen, zoals het creëren van een veilige omgeving in het klaslokaal om seksualiteit te bespreken;

e) Ondersteuning bij het uitvoeren van LLL, specifiek met volledigheid en getrouwheid;

f) Een bredere implementatiestrategie om het implementatieproces van LLL te stimuleren.

Ondanks deze inspanningen lijkt, in het echte leven, absolute volledigheid en getrouwheid van (seksuele) gezondheidsbevorderende schoolprogramma's onrealistisch: aanpassingen komen vaak voor en zijn soms onvermijdelijk. Omgevingsfactoren buiten de controle van docenten kunnen ook de mate waarin een programma wordt geïmplementeerd beïnvloeden en daarmee moet rekening worden gehouden. Docenten moeten daarom worden ondersteund bij het uitvoeren van programma's met zo veel volledigheid en getrouwheid als mogelijk, met begeleiding bij het uitvoeren van de belangrijkste programmaonderdelen om ervoor te zorgen dat de effectiviteit van het programma wordt behouden. Dit vereist creativiteit van ontwikkelaars van het programma; om docenten tegemoet te komen en om volledigheid en getrouwheid van implementatie zo veel mogelijk te bevorderen.

Doorlopende wijzigingen binnen organisaties en in de omgeving vragen voortdurende aanpassing van de implementatiestrategie en het LLL-programma zelf, naar actualiteit, veranderende omstandigheden, de behoeften en verlangens van programmagebruikers en implementeerders, en de interventiecontext om de continuïteit ervan te waarborgen. Dit illustreert dat implementatie een dynamisch proces is. Een succesvolle implementatie van LLL zal uiteindelijk moeten leiden tot jongeren die beter voorbereid, gemotiveerd, en in staat zijn om veilige seks te hebben binnen wederzijdse, aangename relaties. 
Acknowledgements 



\section{ACKNOWLEDGEMENTS}

Finally, we come to a chapter in this dissertation without the word 'implementation' in it! This may be the most important chapter; not only is it usually the most read, but it is also the space where all those are acknowledged for being a part of this marvelous journey and for playing a crucial role in bringing this dissertation to a successful completion. Keeping texts short and concise has not always been my strongest asset, as my promoters will contest, but let's give it a try.

I undertook this $\mathrm{PhD}$ project a few years ago with an optimistic attitude at first, along with some uncertainty, and mainly enthused by ambition. After bags of chocolate M\&M's, paper-covered desks, public transport rides to all corners of the country to provide trainings for the Municipal Health Service (MHS) professionals, feeling like a 'Long Live Love' (LLL) missionary at times, here we are today and I can only look back with a smile. Each step of the way, I had a strong team behind me, and beside me to realize what was set out to be accomplished.

This dissertation would not be complete without the special mention of Prof. dr. Herman Schaalma, the colorful man behind LLL and my master thesis supervisor, where this all began. He was an inspiration, the perfect blend of admirable expertise and tasteful humor, and an absolute joy to be around. You are missed and I hope this dissertation does justice to the legacy you left behind.

To my promoters, Prof. dr. Gerjo Kok and Dr. Fraukje E.F. Mevissen: we did it! This was a great team, where each one's expertise complemented the others'. I am so grateful to have had you both as my promoters, which has allowed this to be a smooth and pleasant project from start to finish. Thank you for all the time and effort you put into me, and the work we produced. Gerjo, not only am I deeply impressed by your revolutionary accomplishments, work, and uncanny ability to get things done and organized but also by the degree of humility, openness, approachability, and kindness that come with who you are as a person. Who knew that a small piece of dark chocolate could make you light up? Fraukje, your eye for detail, effortless ability to see structure in what, at the time, seemed like a jumble of words, and your exceptional strive for nothing less than the best are what make you a formidable co-promoter, and professional. Not only does your constructive feedback stimulate critical reflection but your genuine care for the person as a whole, your encouragement, and support, your willingness to go above and beyond for your PhD students, and your great sense of humor and joyful personality are fantastic qualities that made it a great pleasure to work with you, and produce the articles we have. 
I wish to thank the members of my assessment committee, Prof. dr. Rob Ruiter, Prof. dr. Stef Kremers, Prof. dr. Ilse Mesters, Prof. dr. Guy Parcel, and Dr. Mireille Wolfers, for generously offering their time and willingness to review the dissertation.

To my paranymphs, who are also co-authors of several articles, Suzanne Meijer and Marieke van den Borne: my heart is filled with happiness to celebrate this moment with you, my partners-in-crime, team 'Kwik-Kwek and Kwak'. This truly is and feels like a celebration of our work as a team around LLL and of the realization of this dissertation. It's wonderful to be so aligned in our way of thinking and working. It's been an honor and such a delight to work, laugh and work some more with these two intelligent, expert, thorough, passionate, amazing women. Suzanne, a.k.a. 'Tanti', I can never thank you enough for always believing in me, encouraging me, standing in my corner and being by my side from the very beginning, personally and professionally. You are an extraordinary woman in every aspect and it's an absolute blessing to have you in my life. Marieke, I've never met a harder working, more compassionate, involved, and endearing person. Your diligent work has made a compelling contribution to this dissertation. And all the while, we shared a great deal of laughter and unforgettable times. Thank you both and I celebrate you today.

To the co-authors of the articles; Dr. Ree Meertens, Dr. Theo Paulussen and Dr. Pepijn van Empelen, thank you! Theo, especially your exhaustive work on implementation has been the foundation of this dissertation.

My fabulous team and colleagues in the 'Youth program' at Soa Aids Nederland, thank you for being a part of this journey. Jos Poelman, the legend and godfather of LLL: you are a genuine and admirable person, with a splendid passion, creativity and innovation for your work, and it's been a great joy and honor to have worked with you. Thank you. Hanneke Roosjen, you were always excited for me once an article was published. Thank you for your calm presence, friendship and for being more than a colleague to me. Corona van Ginkel, thank you for all the lunch-dates, the multitude of materials you prepared for all the trainings and conferences in our 'LLL suitcases', and for being the fascinating person that you are. Filippo Zimbile, Koenraad Vermey, Erwin Fisser, Febe Deug and all other colleagues and interns who have enriched our work and my experience at Soa Aids Nederland, thank you.

My vibrant colleagues at Maastricht University. You brightened up my stays in Maastricht and I thank you for the times we shared in conversation and laughter. First the 'old skool' bunch: Dilana, Sanne, Irene, Anne, GJ, and Alvin: the conference in Crete was memorable. Alvin, my brother from another mother, I should have listened to you when 
you told me'Liz, start writing'. Thank you for believing in me and always having my back. Thank you Rob, Gerda, Arjan, Bram, John, Cephas, Gill, Stefan, Joachim, Annet, and Eva, and whoever else I may have forgotten. Rik, the IM training we gave in Ethiopia was fun and thank you for the encouragement. Henna, our enlightening conversations are legendary and I thank you for your precious friendship. A special thank you to Mariëlla Muermans for being so dependable, thoughtful and involved.

Thank you Prof. dr. Gerard van Breukelen for your statistical support amidst your busy schedule. Dr. Madelief Bertens, thank you for your involvement in the evaluation of e-coaching. Mireille Wolfers, Marja Koster and Anita Watzeels, thank you for being part of the 'LLL Dreamteam', and the unforgettable pyjama party at Fraukje's place. Annemies Gort for the editing of all material, Alice Verleun for the production, Ontwerphaven for the designs, Saskia Flipsen for the films, Lynn Werlich for assistance in creating e-coaching, and Jitske de Vries for collaboration around the pilot implementation during your time at TNO.

All of this of course would not have been possible without the input, collaboration, and enthusiasm of teachers and MHS professionals. I give praise to teachers who dedicate their time and effort to their students, who have enthusiastically embraced and delivered LLL, and have committed to teaching their students about relationships and sexuality. I also want to thank the MHS professionals for having climbed on board and supported the implementation (couldn't help it!) process of LLL with passion, enthusiasm, and commitment.

To my close friends and family. Ilenia, my best friend and compadre for life, you are remarkable, and I love you! Nelliene, we go way back! Thank you for your dear friendship. Yulya, Tirza, Shoshannah, Fre, Maliyabo, Kees, 'Global villagers' et al., you brighten up my life! Michael, thank you for being an important part of this journey, asante sana. Aria, thanks for your support 'for sure'. My lovely, adoring, all-too amazing siblings: Georgie, Dirky and Yero, you are my greatest gift in life, the joy in my laughter, and the sun in my sky. I love you guys infinitely: the Fabulous Four. Fati, Zeina, Elhadj, merci. Tante Joke, ome Martin, tante Leny, ome Frans, ome Siem, Ingrid en Gerard, bedankt! Mes parents, je ne pourrais jamais vous remercier assez pour m'avoir donnée une vie si belle, des frères et soeurs si merveilleux et adorables et la grace d'etre qui je suis aujourd'hui. Ma famille, je vous adore, je vous aime. Merci milles fois.

I look back with gratitude for being a piece of the puzzle in such an enjoyable, important, meaningful project that I genuinely believe in, and wholeheartedly support. I am deeply humbled for having had the opportunity to work with the teams and people whose 
contribution, dedication, drive, and expertise resulted in this dissertation, and the work that continues beyond it. This undertaking has taught me the value of perseverance, belief in self, and the magic of teamwork and passion. I honor and thank my ancestors before me, for it is on their shoulders that I stand today. There are no limits for every little girl with big ambitions, opportunity for education, and the freedom to dream. 

Curriculum Vitae 



\section{CURRICULUM VITAE}

Lisette Schutte is a Dutch-Mauritanian who was born in Addis Ababa, Ethiopia on the 19th of April 1986. Having started her education in Addis Ababa, at the Sandford International School, she completed her secondary education in Zambia, at the International School of Lusaka. After enjoying an international upbringing and education, in 2009, she obtained her Master's degree in MSc (Hons) of Health Education and Promotion at Maastricht University under the supervision of Prof. dr. Herman Schaalma and Dr. Ree Meertens. In 2011, she started her PhD project, of which the results are presented in this dissertation, at Maastricht University, under a secondment contract with Soa Aids Nederland (STI Aids, Netherlands). She completed her PhD project under the guidance of Prof. dr. Gerjo Kok and Dr. Fraukje E.F. Mevissen. She is currently policy officer at Soa Aids Nederland, focusing on systematic sexual health promotion, intervention development and implementation, in school-based settings and online. 